\title{
Modeling and Identification of the Nociceptive System using Psychophysical Measurements
}




\title{
MODELING AND IDENTIFICATION OF THE NOCICEPTIVE SYSTEM USING PSYCHOPHYSICAL MEASUREMENTS
}

\author{
Huan Yang
}




\section{Graduation committee:}

\section{Chairman and secretary:}

prof.dr. P.M.G. Apers

University of Twente

\section{Promotor:}

prof.dr. S.A. van Gils University of Twente

\section{Co-promotors:}

dr. H.G.E. Meijer University of Twente

dr.ir. J.R. Buitenweg University of Twente

\section{Members:}

prof.dr.ir. P.H. Veltink University of Twente

prof.dr.ir. M.J.A.M. van Putten University of Twente

apl.prof.dr. D. Kleinböhl Universität Mannheim

dr.ir. A.C. Schouten Delft University of Technology

prof.dr. R.J. Boucherie University of Twente

The work presented in this thesis is carried out in the group of Applied Analysis in the MIRA institute for biomedical engineering and technology medicine in the University of Twente. This research is supported by the Dutch Technology Foundation STW, which is part of the Netherlands Organisation for Scientific Research (NWO) and partly funded by the Ministry of Economic Affairs (project number 10740).

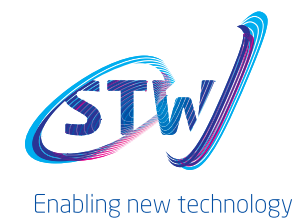

\section{UNIVERSITY OF TWENTE.}

ISBN: 978-90-365-4004-9

DOI: $10.3990 / 1.9789036540049$

Printed by: Gildeprint Drukkerijen

Copyright (C) 2015, Huan Yang, Enschede, the Netherlands.

ALL RIGHTS RESERVED. No part of this publication may be reproduced in any form or by any electronic or mechanical means, including information storage or retrieval systems, without permission from the author. 


\title{
MODELING AND IDENTIFICATION OF THE NOCICEPTIVE SYSTEM USING PSYCHOPHYSICAL MEASUREMENTS
}

\section{DISSERTATION}

\author{
to obtain \\ the degree of doctor at the University of Twente, \\ on the authority of the rector magnificus, \\ prof.dr. H. Brinksma, \\ on account of the decision of the graduation committee, \\ to be publicly defended \\ on Friday 4 December 2015 at 14:45 hrs \\ by \\ Huan Yang
}

born on 29 December 1985

at Liaoyang, China 
This dissertation has been approved by

$$
\begin{array}{lll}
\text { promotor } & : & \text { prof.dr. S.A. van Gils } \\
\text { co-promotors } & : & \text { dr. H.G.E. Meijer } \\
& \text { dr.ir. J.R. Buitenweg }
\end{array}
$$




\section{Contents}

1 Introduction 1

2 Computational modeling of Adelta-fiber-mediated no$\begin{array}{ll}\text { ciceptive detection of electrocutaneous stimulation } & 17\end{array}$

2.1 Introduction . . . . . . . . . . . . . . . . . 19

2.2 Psychophysical human-subject experiment . . . . . . . . . . . . 21

2.2 .1 Methods . . . . . . . . . . . . . . . . . . . . . 21

2.2 .2 Results . . . . . . . . . . . . . . . . . . . 22

2.3 Computational modeling . . . . . . . . . . . . . . . . . 23

2.3.1 Activation of afferent fibers . . . . . . . . . . . . . 24

2.3 .2 Post-synaptic dynamics . . . . . . . . . . . . . . . . 26

2.3.3 Stimulus detection by randomly spiking secondary neurons . 27

2.3 .4 Lumped models . . . . . . . . . . . . . . . . . . . . . . . 29

2.3.5 Comparison of the dynamics and psychophysical functions of DDM and $\mathrm{HM} \ldots \ldots \ldots \ldots \ldots \ldots \ldots$

2.4 Effects of temporal stimulus properties on detection thresholds . . . 36

2.5 Discussion . . . . . . . . . . . . . . . . . . . . 39

2.5.1 Effects of temporal properties on detection thresholds . . . . 39

2.5.2 Interpretation of lumped parameters . . . . . . . . . 40

2.5 .3 Model identifiability . . . . . . . . . . . . . . . . . 41

2.5.4 Model extensions . . . . . . . . . . . . . . . . . . . . . . 42

3 Dependence of nociceptive detection thresholds on physiological parameters and capsaicin-induced neuroplasticity: a computational study 45

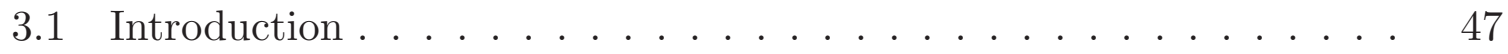

3.2 Material and methods . . . . . . . . . . . . . . . . . 49 
3.2.1 Computational modeling of nociceptive detection of electrocutaneous stimulation . . . . . . . . . . . . . 49

3.2.2 Effects of single model parameters on detection thresholds . . 51

3.2.3 Effects of temporal stimulus properties on detection thresholds 52

3.2.4 Neuroplasticity induced by topical capsaicin treatment and relevant modeling . . . . . . . . . . . . . . . 54

3.3 Results. . . . . . . . . . . . . . . . . . 56

3.3.1 Effects of single model parameters on detection thresholds . . $\quad 57$

3.3.2 Effect of the IPI on detection thresholds . . . . . . . 57

3.3.3 Simulated detection thresholds after topical capsaicin treatment 58

3.4 Discussion . . . . . . . . . . . . . . . . . . . . . . . 59

3.4.1 Effects of parameters on detection thresholds . . . . . . . 60

3.4.2 Modeling capsaicin-induced plasticity and its interpretation . 62

3.4.3 Future studies on capsaicin-induced neuroplasticity . . . . . . 64

4 Estimation and identifiability of model parameters in human nociceptive processing using stimulus-response pairs

4.1 Introduction . . . . . . . . . . . . . . . . . . . . 69

4.2 Experiments and models . . . . . . . . . . . . . . . . 72

4.2.1 Nociceptive detection task and datasets with stimulus-response pairs . . . . . . . . . . . . . . . 72

4.2 .2 The logistic regression model . . . . . . . . . . . . . 74

4.2 .3 The hazard model . . . . . . . . . . . . . . . . . . . 74

4.3 Model fitting and evaluation . . . . . . . . . . . . . 76

4.3.1 Optimal model fitting of the HM by maximizing the likelihood

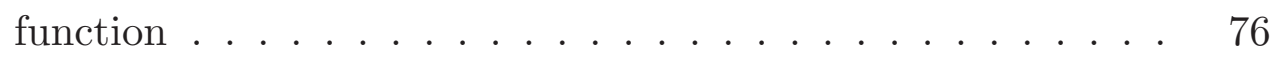

4.3.2 The balance between model fit and complexity . . . . . . 78

4.3 .3 Results . . . . . . . . . . . . . . . . . . . . . . . 79

4.4 Identifiability analysis $\ldots \ldots \ldots \ldots \ldots$

4.4.1 Profile likelihood approach _. . . . . . . . . . . 81

4.4.2 Structural and practical non-identifiability in the HM $\ldots 83$

4.4 .3 Results . . . . . . . . . . . . . . . . . . . 87

4.5 Discussion . . . . . . . . . . . . . . . . . . . . . 90 
5 Threshold estimates from logistic regression on stimulusresponse pairs from staircase procedures with a case study of a nociceptive detection task $\quad 99$

5.1 Introduction . . . . . . . . . . . . . . . . . . . . . . 101

5.2 Staircase procedures in a nociceptive detection task . . . . . . . 103

5.3 Markov models for staircase procedures and their properties . . . . . 104

5.3.1 Markovian formulation . . . . . . . . . . . . . . . 105

5.3.2 Relation between SSP and APP . . . . . . . . . . . 106

5.3.3 Asymptotic sampling distributions of reference point and applied amplitude . . . . . . . . . . . . . . . . . 108

5.4 Cases studies for the nociceptive detection task . . . . . . . . . 111

5.4.1 Model-based psychometric functions . . . . . . . . . . . 111

5.4.2 Simulations of asymptotic sampling distributions . . . . . . . 114

5.5 Threshold estimates for staircase procedures and their statistical biases 115

5.5 .1 Logistic regression . . . . . . . . . . . . . . 116

5.5.2 Asymptotic characteristics of threshold estimates . . . . . 117

5.5.3 Estimation of thresholds with finite samples of stimulus-response pairs . . . . . . . . . . . . . . . . 119

5.6 Conclusions and discussion $\ldots \ldots \ldots \ldots$

6 Discussion, Outlook and Concluding Remarks $\quad 137$

$\begin{array}{ll}\text { References } & 158\end{array}$

$\begin{array}{ll}\text { Summary } & 159\end{array}$

$\begin{array}{ll}\text { Samenvatting } & 161\end{array}$

$\begin{array}{ll}\text { Acknowledgments } & 163\end{array}$

$\begin{array}{ll}\text { About the Author } & 165\end{array}$

$\begin{array}{ll}\text { List of Publication } & 167\end{array}$ 


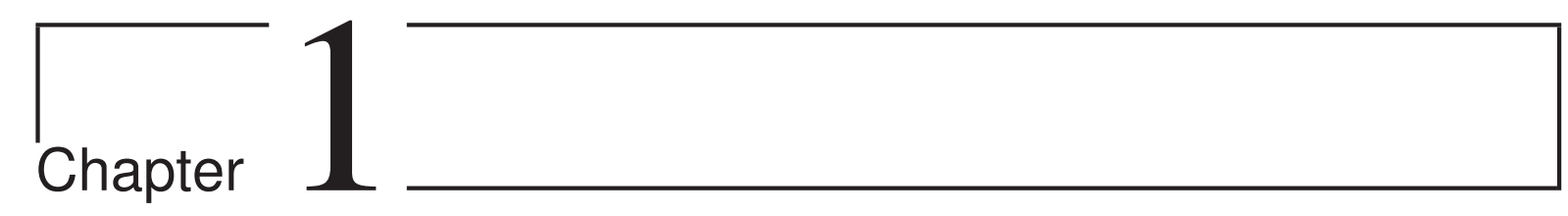

\author{
Introduction
}

\title{
An 'eye chart' to diagnose pain disorders?
}

Humans have multiple senses, which are involved in interactions with the environment, e.g. reading this page of the thesis. Abnormality in related sensory function will reduce the quality of life unless proper treatment is implemented. For example, for 25 percent of world's population with nearsightedness (Czepita, 2002), a convenient routine often works: by wearing appropriate corrective lenses based on visual acuity tests with an eye chart. However, for a comparable population suffering from pain disorders (Tsang et al., 2008), efficient pain management is often obstructed by lack of reliable diagnosis (Wall and Melzack, 1989). Hence, a healthcare challenge arises: can we diagnose states of pain-related systems in a similarly convenient way as the eye-chart test, which could facilitate further improved pain management? On the way to address that, this thesis aims to offer the foundation for such diagnostic tools. 


\section{Pain, nociceptive function and plasticity}

The International Association for the Study of Pain (IASP) defines pain as an unpleasant sensory and emotional experience associated with actual or potential tissue damage, or described in terms of such damage (see this definition on the IASP homepage: www.iasp-pain.org). Pain is essential to survive. Being able to feel pain can help humans to prevent further severe tissue damage. This is often referred to as acute pain, whose state usually diminishes as the injured tissues heal. As a multifactorial phenomenon, pain involves sensory, emotional and cognitive aspects. However, from an aetiological perspective, understanding how the peripheral and central nervous system process an actually or potentially tissue-damaging event, i.e. nociceptive function, could facilitate improved therapeutics against pain disorders in humans (Woolf and Mannion, 1999).

In contrast to acute pain, chronic pain is a disorder with persistent pain even after the tissue damage has worn off. It brings substantial burden to both individuals and society. Once a patient has chronic pain, efficient treatment is often difficult because the cause is not known. The general biological explanation is that maladaptive nociceptive function can lead to the development of chronic pain states (Latremoliere and Woolf, 2009; Sandkühler, 2009). Similar adaptive capabilities in other parts of the nervous system explain life-long learning for humans during healthy development. Such (mal-)adaptive processes are known as neuroplasticity. Nociceptive neuroplasticity is often caused by nerve injury, inflammation or disease. In view of biological scales, neuroplasticity ranges from genetic to behavioral levels.

Conventional clinical practice typically employs a symptom-based diagnosis with patients' reports of pain, like history, intensity and location of pain. This diagnosis provides a general practitioner or pain specialist with a brief impression about states of patients' pain. However, the mere usage of patient self-reports may blur the overall picture of underlying nociceptive function. For therapeutic purposes, assessment of nociceptive function provides more direct insights into neuropathology of the underlying nervous system. For pain medicine, this promotes a paradigm shift with mechanism-based diagnosis which can more specifically assess nociceptive function.

To achieve such a paradigm shift, clinically applicable and non-invasive observation techniques are under development. As a myriad of processes contribute to 
the overall human nociceptive function, meaningful interpretation of those observations differentiates between mechanisms, is not straightforward. In this thesis, computational modeling studies aim to translate observations into insightful characteristics of nociceptive processes. In the next section, we give an overview of the human nociceptive system and measurement techniques. These form the basis for our model-based studies towards differential diagnosis.

\section{Human nociceptive system: mechanisms and mea- surements}

Nociceptive mechanisms can be studied at different biological scales. They range from genes and molecules (Akopian et al., 1996; Tolner et al., 2015), cells and tissues (Lee et al., 2005), to organs and entire animal/human function (Rolke et al., 2006). There are many fundamental and translational studies, whose ultimate goal is to prevent and alleviate pain disorders of humans. In this section, we will start with an overview of essential nociceptive mechanisms in the human nociceptive system under both physiological and pathological conditions. Next, we motivate the applicability of psychophysical techniques to provide information about these mechanisms.

\section{Neurophysiology and neuropathology}

Roughly speaking, the human nociceptive system consists of peripheral and central subsystems. First, the peripheral subsystem involves nociceptive nerves: unmyelinated $\mathrm{C}$ fibers and thinly myelinated A $\delta$ fibers (Wall and Melzack, 1989). Free nerve endings are the peripheral terminals of nociceptive nerves and penetrate mostly in the epidermal layer of the skin (Granstein and Luger, 2009). These endings are equipped with various ion channels, which can transduce nociceptive stimuli into neural activity (Purves, 2008). Excitability characterizes the overall function of endings in response to applied stimulation. When the free nerve endings are excited, i.e. action potentials are generated, nerve fibers convey the initiated excitation to their central terminals at the dorsal horn in the spinal cord. The extent of myelination of a fiber determines its conduction velocity. Fast pain travels via myelinated A $\delta$ fibers with velocities from 15 to $30 \mathrm{~m} / \mathrm{s}$, which is higher than that of unmyelinated $\mathrm{C}$ fibers conveying slow pain. Second, in the central subsystem, dorsal horn 
neurons receive and process synaptic inputs, where both synaptic efficacy and its neuronal excitability determine the postsynaptic activity. Third, the resulting activity is relayed to supraspinal cites, e.g. the thalamus and the somatosensory cortices via the anterolateral system (Purves, 2008). Fig. 1.1A presents a schematic diagram of the essential peripheral and central nociceptive subsystems.

In the spinal neuronal circuits, projection neurons in the superficial laminae are well studied, see the review by Todd (2010). These superficial laminae are also the major termination region for $\mathrm{C}$ and $\mathrm{A} \delta$ fibers, see a schematic diagram in Fig. 1.1B. In addition to nociceptive transmission from the periphery, the spinal nociceptive system can receive descending controls from supraspinal sites (Yarnitsky, 2010). Under normal conditions, such descending controls only become effectively active by applying intense stimuli to a human subject (Millan, 2002).

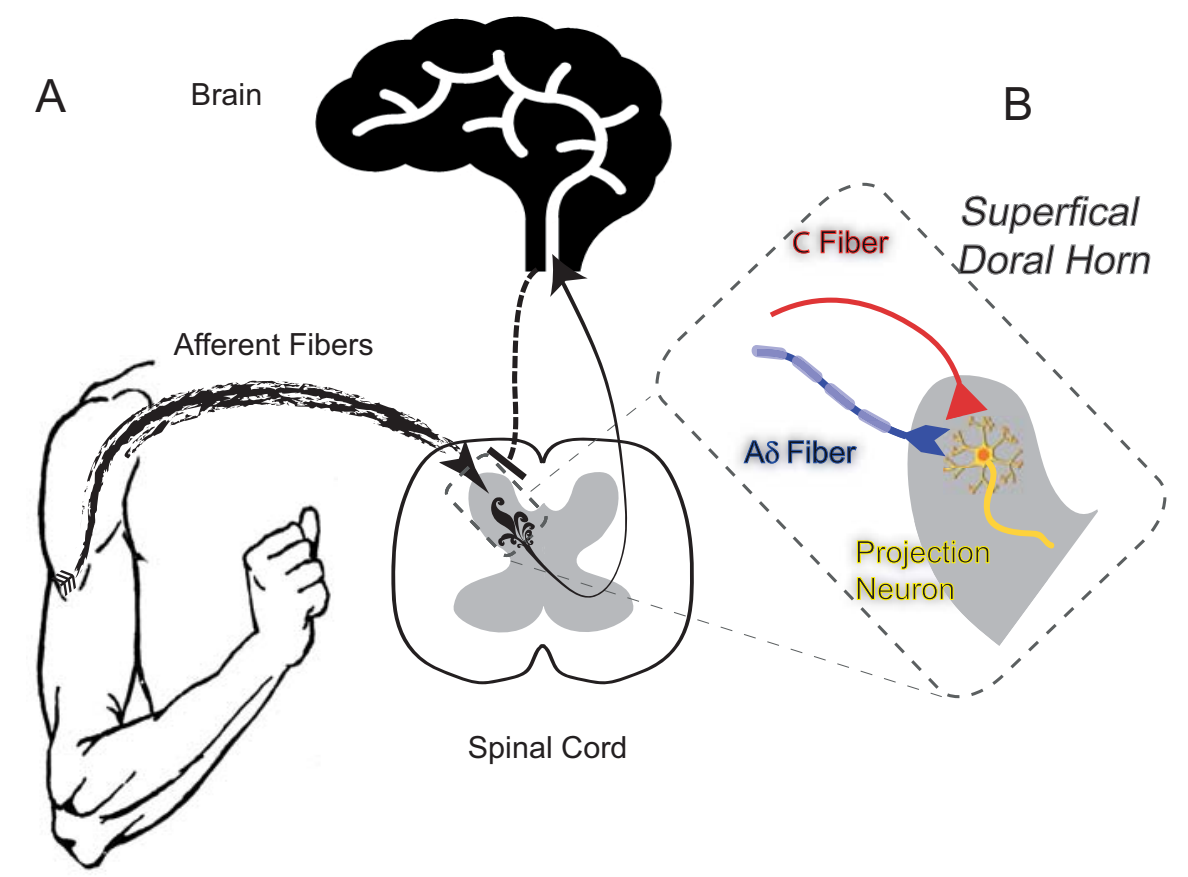

Figure 1.1: The nociceptive system. A: Schematic diagram of the peripheral and central nociceptive subsystems; B: Synaptic contacts of central terminals of A $\delta$ and $\mathrm{C}$ fibers to projection neurons within the superficial dorsal horn of the spinal cord.

Nociceptive subsystems, alike most other nervous systems, are subject to plasticity, i.e. the system adapts in response to environmental changes. It has been hypothesized that chronification of pain states is associated with various forms of neuroplasiticty (Sandkühler, 2007; Latremoliere and Woolf, 2009). However, it is still poorly understood how the acute pain phase turns into a chronic phase after 
nerve injury or surgery (Ruscheweyh et al., 2011; Voscopoulos and Lema, 2010). Hyperalgesia, as a symptom or disease, indicates pain hypersensitivity. Experimental and clinical studies often assess this hypersensitivity by observing increased pain from a stimulus that normally provokes pain (Sandkühler, 2009). Nociceptive sensitization is responsible for this phenomenon (Coutaux et al., 2005; Latremoliere and Woolf, 2009), involving both central and peripheral neuroplasticity. As multiple nociceptive subsystems are involved, to identify which ones are malfunctioning is necessary for a more efficient pain treatment. In addition, the way how the subsystems are malfunctioning deserves further investigations. Although nociceptive sensitization is similar to long-term potentiation underlying memory, nociceptive sensitization involves a myriad of molecular processes. Many of these processes remain not fully characterized, in particular not for central sensitization (Ji et al., 2003; Sandkühler, 2010; Latremoliere and Woolf, 2010).

Given the multifactorial nature of pain, in a clinical setting, other symptoms of patients could further challenge identifying the nociceptive subsystem that does not work properly. Experimental paradigms in controlled circumstances could remedy this. Several human experimental pain models have been proposed to measure stimulus-response relations to reveal the underlying nociceptive mechanisms (Olesen et al., 2012). The stimulus can be of phasic or tonic type (Granot et al., 2003; van der Heide, 2009), where the former often spans much shorter intervals than the latter. Phasic stimuli usually play a predominant role to activate the nociceptive system (Sinke et al., 2015). However, exposure to tonic stimuli can modulate and even modify the nociceptive system, resulting in various structural and functional forms of neuroplasticity (Woolf and Salter, 2000). Given the specific organization of the innervation of central terminals as presented in Fig. 1.1B, various forms of central plasticity in the superficial dorsal horn are thought to contribute to prolongation of pain states.

Induction of central sensitization requires an intense, repeated and sustained nociceptive stimulus (Latremoliere and Woolf, 2009). This can be achieved in various ways by application of capsaicin, which is the pungent substance in chili peppers (O'Neill et al., 2012). For example, topical application of capsaicin is used as an experimental human pain model altering the normal nociceptive function, accompanied by changes in peripheral and central nociceptive properties. In this case, the first action is that capsaicin activates $\mathrm{C}$ and some $\mathrm{A} \delta$ fibers via the transient receptor potential vannilloid 1 (TRPV1). Second, capsaicin causes structural neu- 
roplasticity in periphery, namely loss and retraction of nerve endings (Anand and Bley, 2011; O'Neill et al., 2012). Third, activation of C fibers triggers the release of neuropeptides in both peripheral and central terminals. This leads to functional neuroplasticity, i.e. enhanced synaptic efficacy and increased excitability of both peripheral nerve endings and dorsal horn neurons (Coutaux et al., 2005; Todd, 2010). These induced forms of neuroplasticity have different time courses, from hours to days and possibly longer.

\section{Psychophysical approaches}

To study sensory function, psychophysical approaches measure a subject's sensation and performance to applied physical stimuli. These methods require conscious and cooperative human subjects. On the other hand, psychophysical techniques have lower cost and higher convenience than other neurophysiological techniques, like electroencephalogram or functional magnetic resonance imaging (Olesen et al., 2012). Psychophysical approaches are often referred to as 'outer psychophysics' (Fechner, 1860). From an alternative point of view, this involves two steps. The first step is to relate physical stimulation to neural activity, which involves neurophysiology. A second step is to translate the resulting activity to subjective sensation known as 'inner psychophysics', which is brought forth by Fechner (1860). Fig. 1.2 illustrates the 'outer psychophysics', and its intermediate route with both neurophysiology and the sequential 'inner psychophysics'.

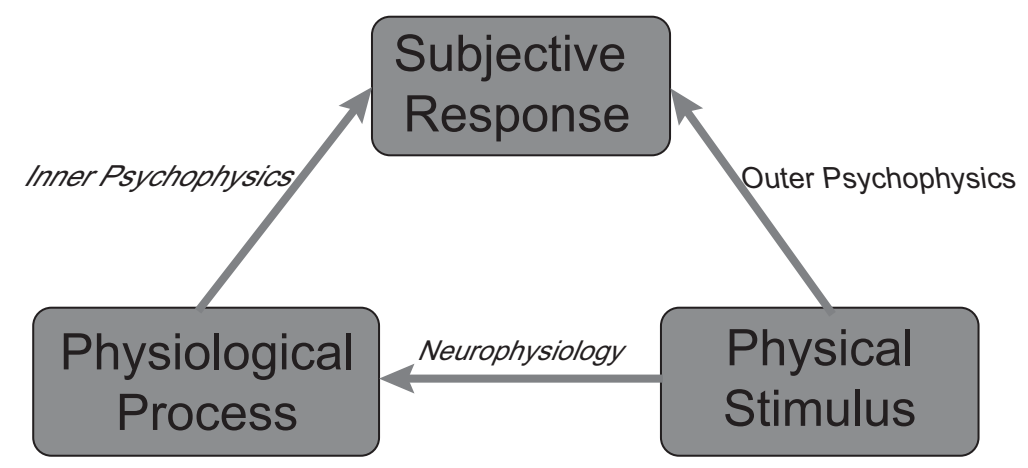

Figure 1.2: Psychophysical approach in a broad sense (adapted from (Fechner, 1860)).

To assess pain-related and nociceptive function, quantitative sensory testing has been developed (Rolke et al., 2006). Its clinical applicability was advocated (WilderSmith, 2002). One should choose a relevant stimulus modality to assess a specific 
nociceptive (sub)system. There are various stimulus modalities, e.g. cold, hot, and chemical. Research in this thesis considers electrocutaneous stimulation, as it has the following advantages. First, free nerve endings mainly locate in the epidermis, which is more superficial than non-nociceptive afferents, e.g. A $\beta$ fibers. Several works reported that at a low intensity, electrocutaneous stimulation could selectively recruit A $\delta$ fibers by intra-epidermal electrodes (Inui et al., 2002; Mouraux et al., 2010). Such low-intensity stimulation, typically of a phasic type, is compatible with detection tasks. Second, temporal properties are more easily adjustable for electrical stimuli than natural stimuli, e.g. heat stimuli. This advantage offers possibilities for investigating peripheral and central subsystems with distinct time constants. Third, considering the spinal neuronal circuit sketched in Fig. 1.1B, both heterotopic and homotopic stimulation of tonic type can serve as 'conditioning stimuli' to modulate studied nociceptive function (Haefeli et al., 2014). For example, application of a tonic and homotopic high-frequency stimulation can result in volleys of C-fiber-mediated activities (Latremoliere and Woolf, 2009). Hence, for healthy human subjects, an experimental paradigm with collaborative use of phasic and tonic stimuli enables to assess nociceptive function under both normal and (mal-)adaptive conditions.

A detection trial forms the basis of the psychophysical experiment by applying electrocutaneous stimulation and measuring the binary detection response. A set of stimulus-response pairs is often collected sequentially with adaptive procedures, e.g. non-parametric staircase procedures (Treutwein, 1995; Lu and Dosher, 2013). The observed stimulus-response pairs provide information about the underlying sensory function. An important characteristic is the detection threshold, i.e. the stimulus amplitude, corresponding to 0.5 detection probability. By convention, psychophysical studies often utilize these detection thresholds to understand various sensory functions (Ehrenstein and Ehrenstein, 1999; Goldstein, 2009; Kingdom and Prins, 2010).

\section{PAINSIGHT}

This thesis is based on research conducted in the project "PArameter Identification of the Nociceptive System for Improved monitorinG of cHronic pain developmenT" (PAINSIGHT). The PAINSIGHT project is one of the innovative and multidisciplinary projects within the consortium System Identification and Parameter Estimation of Neurophysiological Systems (NeuroSIPE), which is supported by 
the Dutch Technology Foundation STW. All NeuroSIPE projects aim to offer diagnostic tools for various neurological disorders. The PAINSIGHT project has its own mission to develop a clinically applicable diagnostic tool for (mal-)functioning of the nociceptive system during potential transition of acute pain into chronic pain states. The work presented in this thesis focuses on a mechanism-based interpretation of psychophysical observations towards a differential diagnostic tool, where we develop computational approaches in tandem.

\section{Computational approaches}

Computational approaches aim to integrate computational modeling with experimental or clinical observations for explanatory characteristics of underlying nociceptive processes. Such integration requires compatibility between computational models and observations.

\section{Where do we start modeling, from data or from principles?}

Different from relatively mature disciplines like classical physics, neuroscience is relatively new. Understanding of the nervous system is still rapidly increasing thanks to observation techniques like patch clamping (Hamill et al., 1981), functional imaging (Friston, 1994) and the recently developed optogenetics (Carr and Zachariou, 2014). Observed phenomena often help to understand the underlying system. However, counterintuitive phenomena may challenge current understandings. Now, computational modeling enters the scene to solve such puzzles. Mathematical abstraction often translates current understandings, i.e. principles, into formulas. For example, cable theory with mathematical models was established to understand processing in dendritic neurons (Koch and Segev, 1998). Mathematical modeling and analysis can increase understanding of biological processes, offer insight in design of future experiments, and detect abnormalities of current knowledge of the biological system. We give a brief survey of basic aspects to model real-life systems.

\section{Static and dynamic systems}

A static system is memoryless, i.e. the previous states have no impact on the current and future states of the system. For example, a homogeneous medium can 
be considered as a static system, where a point charge and the induced electric potential field are the system's input and output, respectively. Then, their relation is formulated by an algebraic expression. In contrast, the state of a dynamic system evolves in time. For continuous-valued and continuous-time states, the system can be mathematically formulated as a set of differential equations. For example, Hodgkin and Huxley (1952) formulated a hallmark model with a set of ordinary differential equations for excitable neurons.

\section{Linear and nonlinear systems}

Input-output systems are often classified as linear or nonlinear systems. Roughly speaking, the output of a linear system is proportional to the input and obeys superposition (Westwick and Kearney, 2003). Such rules are not applicable to a nonlinear system. This classification is often used for dynamic systems. Within a suitable regime, linearization often simplifies the nonlinear system as a linear one.

\section{Deterministic and stochastic systems}

If a system is deterministic, the governing law will be free of randomness. For example, fixing the initial conditions and parameter values, simulations of the HodgkinHuxley model (Hodgkin and Huxley, 1952) will show the same dynamics. On the contrary, a stochastic system can produce different outputs even with identical inputs and pre-defined intrinsic properties of the system. For example, the observation error is a frequently used stochastic model, which follows a normal distribution. For the nervous system, sources of stochasticity exist in various stages of neuronal processing, e.g. the probabilistic states for opening or closing of ion channels or synaptic noise (Faisal et al., 2008).

\section{Existing computational models of nociceptive processing}

Current understandings of neurophysiology and neuropathology serve as basic principles to build computational models of nociceptive processing. Here, we review existing computational models of nociceptive processing in various contexts.

First, Britton and Skevington (1989) developed a model of the gate control theory of pain (Melzack and Wall, 1965). They focused on nociceptive processing 
of spinal neurons with impulses conveyed by both thinner nocicpetive afferents $(\mathrm{A} \delta$ and $\mathrm{C}$ fibers) and thicker non-nociceptive $\mathrm{A} \beta$ afferents. More recently, the modeling study (Farajidavar et al., 2008) focused on spinal neurons to understand short-term plasticity, known as the wind-up effect. Although these studies incorporated synaptic inputs of different kinds of primary afferents in their models, an interface on nerve excitation was missing. Lack of an interface hampered the models' compatibility with experiments, which are conducted on an intact animal or human subject. Second, Mørch et al. (2011) conducted a detailed modeling work for the nerve interface for electrical stimulation by surface electrodes. Their modeling took geometric features of afferents in the cutaneous tissue into account. Simulation results suggested the dependence of activation of different afferents on geometric properties of electrodes and the stimulus intensity. However, no further study has integrated this modelled interface to investigate nociceptive function of intact human subjects yet. Third, as a holistic modeling work, Xu et al. (2008a) focused on pain sensation to a thermal stimulus, incorporating both peripheral and central stages of nociceptive processing. This model contained a large set of parameters, whose values were based on literature rather than constrained by experimental data.

In short, all above models with different focuses provided various theoretical implications regarding neuronal processing in peripheral or central nociceptive subsystems. However, there are few further applications of these models, partially attributed to lack of applicability on further testing of model-based implications (Argüello et al., 2015). To address that, one prerequisite is to develop clinically applicable observation techniques. Together with the above mentioned psychophysical techniques, we envisage that a model-based study will not only explain observed phenomena but also generate new testable hypotheses. We observe that the existing models are incompatible with the detection task with electrocutaneous stimulation. The task involves a different interface and possibly recruits different central neurons. So, a compatible and plausible model should be developed for the detection task.

\section{Modeling the nociceptive detection of electrocutaneous stim- ulation}

The underlying nociceptive system consists of peripheral and central nociceptive subsystems. To characterize these subsystems, computational models are expected to contain multiple neurophysiologically meaningful parameters. These subsystems 
could be modelled in different aspects, which we surveyed above. Hence, it is still an open and challenging question how to build a plausible model for the detection task. First of all, there is no doubt that deterministic physical principles in nerve activation and central neuron processing should be incorporated into the modeling. Involved parameters are expected to characterize biological processes, like excitability or synaptic efficacy. Second, because of the stochastic nature of binary responses, the computational model is expected to involve some stochastic processes. Third, different subsystems can have distinct time scales. On one hand, some subsystems that evolve on relatively slow time scales could be further approximated as static. One the other hand, such static subsystems are often nonlinear, yielding a cascade of dynamic subsystems with static nonlinearity, known as Wiener and Hammerstein systems (Billings, 1980; Marmarelis, 2004). Hence the overall model will still be nonlinear.

\section{Qualitative validation of models}

When a computational model is proposed, it is often criticized as the mathematical abstraction cannot capture the real-life observations. From a system point of view, observations are (partially) collected from the target biological system under different conditions. Alternatively, some empirical rules can be considered to validate the developed model. For example, probability summation is a widely accepted rule in psychophysics (Quick Jr, 1974; Watson, 1979; Tyler and Chen, 2000).

Because experiments providing observations could be (extremely) time consuming, model criticism should be incorporated when new observations are available. If one detects a substantial mismatch between observations and model replication, one should refine the model by reconsidering principles until achieving a satisfactory match. After that, it is time to conduct a new experiment to collect observations for further criticism on the (refined) model. Such an iterative process progressively increases the model's plausibility with wider applicability driven by both principles and observations.

Psychophysical studies typically provide averages of detection thresholds from a group of human subjects as empirical data (Moscatelli et al., 2012). Further statistical analysis of the averages can increase our understanding of the underlying sensory function (Gold and Ding, 2013). As a qualitative validation of a model, one can compare the model-simulated detection thresholds with averages of thresholds 
obtained under different conditions. These conditions could be sequential phases within a human experimental pain model, where modulatory effects induced by tonic stimulation or medical intervention on the nociceptive system usually last for a relatively long period. Also, by checking the compatibility with empirical psychophysical rules, one can qualitatively validate the model.

\section{System identification and parameter estimation}

We aim to build a plausible computational model to represent both peripheral and central subsystems. In a simulation study, a model can produce realistic model predictions with plausible values of parameters. For example, model simulations of the Navier-Stokes equations increase the understanding of fluid mechanics (Constantin and Foias, 1988). However, for biological systems, the characteristics of these systems are often poorly understood. This is often attributed to the fact that values of these parameters could vary among different species or inter-individually due to biological variability. For the nociceptive system under study, this is reflected in unknown values of parameters in the peripheral and central subsystems for an individual subject.

From a qualitative validation of a developed model, satisfactory outcomes do not imply that the model can meet the requirements for diagnostic purposes. To bridge the gap, it requires a more quantitative step. That is an integration of stimulusresponse pairs measured from an individual subject with the developed model to estimate values of parameters. This integration strategy has been extensively applied in several biomedical applications, e.g. systems biology (van Riel, 2006) and biomechanics (Kearney and Hunter, 1989). In general, parameter estimation often starts with an objective function. This objective function describes the goodness of fit by the model with unknown parameter values to measurements. One can determine the point estimates of parameters, which is often achieved by optimizing the objective function. With specific objective functions, different point estimation methods have been proposed, such as maximum likelihood estimation or (weighted) least square methods. In contrast, interval estimations calculate intervals of values of model parameters with observations. In practice, the combined results from both point and interval estimations are often reported, e.g. the mean of the grouped data and the standard error of the mean.

In a typical setting to estimate parameters, measurements of a dynamic system 
consist of samples of the system dynamics together with system inputs, if applicable. For example, within a suitable measuring regime, Fors et al. (1984) developed a linear model to represent the relation between the intradental nerve activity and time-varying pain-related behavior. They applied classical techniques to estimate model parameters. For more general situations, both model structures and types of measurements challenge identification and estimation. For various types of (nonlinear) systems with continuous-value measurements, advanced algorithms have been developed (Hunter and Korenberg, 1986; Pintelon and Schoukens, 2012). For the setting with continuous-time but quantized measurements, novel system identification approaches have been initiated (Zhao et al., 2007; Wang et al., 2010). However, these existing techniques are not compatible with measurements in the nociceptive detection task, as the response is a single binary value. Here, the first challenge is to provide point estimates of parameters with measured stimulus-response pairs. In addition, another challenge is to recover the unambiguous values of parameters, which implies to narrow the interval estimate. Hence, both point and interval estimation approaches need to be developed or adapted to be compatible with relatively limited measurements from the detection task.

When a model replicates the observations well and offers unambiguous parameter estimates, in turn, such a model could be valuable to understand the underlying neuronal mechanisms or to design future experiments.

\section{Aim and outline of this thesis}

The general aim of this thesis is to increase our understanding of $\mathrm{A} \delta$-fibermediated nociceptive processing, in particular with model-based interpretations of psychophysical observations in normal and perturbed conditions. For this, we define the following main objectives:

1 To develop computational models of a nociceptive detection task with physiologically meaningful parameters quantifying states of peripheral and central nociceptive subsystems.

2 To demonstrate the model's ability in replicating psychophysical characteristics and in turn to advance our understanding of nociceptive processing with various forms of neuroplasticity induced by a high-dose capsaicin patch.

3 To integrate the model with experimental stimulus-response pairs to address 
estimation and identifiability of system parameters for an individual subject.

4 To evaluate adaptive procedures regarding the statistical bias of detection thresholds by performing model-based psychophysical experiments.

We address the above objectives in Chapter 2-5. These chapters can be read separately as they have been written as articles for peer reviewed journals.

Chapter 2 develops neurophysiologically plausible models of the nociceptive detection of electrocutaneous stimuli. Each of these model retains six lumped parameters characterizing peripheral and central nociceptive subsystems (objective 1). Qualitative agreements between model predictions and experimentally obtained detection thresholds are achieved, especially the dependence of threshold on three temporal stimulus properties. In turn, these models also offer neurophysiology-based interpretation of phenomena in the normal condition (half of objective 2).

Chapter 3 continues to achieve the remaining half of objective 2 by investigating various forms of neuroplasticity in the nociceptive system. We study effects of single model parameters on detection thresholds. Also, we derive a condition for possible relations between detection thresholds and the interpulse interval for double-pulse stimuli. In a case study with topical capsaicin treatment, we translate neuroplasticity into plausible changes of model parameters. Model-based simulations agree with experimental patterns of changes in detection thresholds over three months. This facilitates to separate different contributions of capsaicin-induced plasticity.

In addition to qualitative agreements between model predictions to experimentally obtained detection thresholds, we continue with a more quantitative study in Chapter 4. To assess states of nociceptive subsystems, we formulate and apply a maximum likelihood approach to provide point estimates of model parameters using stimulus-response pairs measured from an individual subject. In addition, we employ the profile likelihood approach to provide confidence intervals for parameter estimates. This model-based approach addresses the plausibility of the model and parameter identifiability, leading to a potential diagnostic tool to detect malfunctioning in nociceptive subsystems.

Chapter 5 investigates the statistical bias of threshold estimates from logistic regression using stimulus-response pairs sampled from staircase procedures. We formulate a newly developed staircase procedure as a Markov model. This facilitates a theoretical understanding about whether and how asymptotic characteristics of 
the bias depend on procedural parameters in those staircase procedures. Conducting Monte Carlo simulations for various cases with finite samples, we end up with recommendations for the use of adaptive procedures in practice.

Chapter 6 concludes the thesis and recommends further directions based on findings of the doctoral research. 


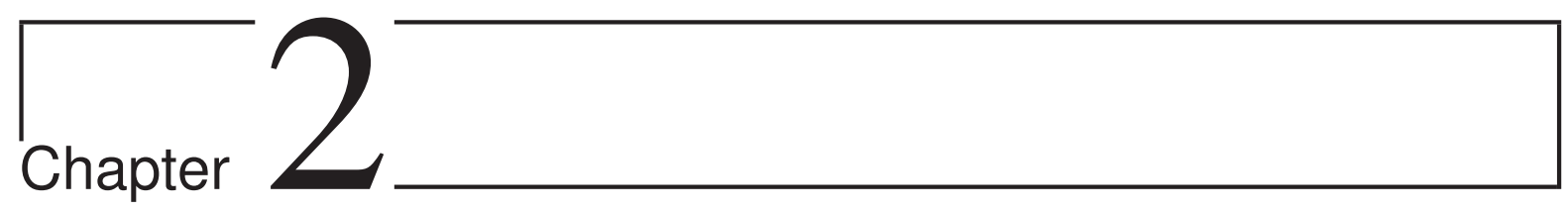

Computational modeling of Adelta-fiber-mediated nociceptive

detection of electrocutaneous stimulation

This chapter is adapted from the work (Yang et al., 2015a). 


\section{Abstract}

Sensitization is an example of malfunctioning of the nociceptive pathway in either the peripheral or central nervous system. Using quantitative sensory testing, one can only infer sensitization, but not determine the defective subsystem. The states of the subsystems may be characterized using computational modeling together with experimental data. Here, we develop a neurophysiologically plausible model replicating experimental observations from a psychophysical human-subject study. We study the effects of single temporal stimulus properties on detection thresholds corresponding to a 0.5 detection probability. To model peripheral activation and central processing, we adapt a stochastic drift-diffusion model and a probabilistic hazard model to our experimental setting without reaction times. We retain six lumped parameters in both models characterizing peripheral and central mechanisms. Both models have similar psychophysical functions, but the hazard model is computationally more efficient. The model-based effects of temporal stimulus properties on detection thresholds are consistent with those from human subject data. 


\section{$2.1 \quad$ Introduction}

Increased insight into neurophysiological mechanisms of the nociceptive pathway may contribute to more reliable monitoring of chronification of pain and patienttailored pain therapies (Dworkin et al., 2003; Baron, 2006). To achieve this goal, a computational model of stimulus processing may be an in-dispensable tool. For instance, the model could provide a mechanism-based interpretation of experimental observations. In turn, this may explain or predict effects of pharmaceutical interventions in the nociceptive system. Another, prospective, use may be to estimate model parameters from measurements. The estimate might inform about the state of the nociceptive system and possibly indicate its malfunctioning, e.g. due to central sensitization, which could result in chronic pain (Latremoliere and Woolf, 2009).

Hyperalgesia is a clinically important example of malfunctioning of the nociceptive system and is characterized as an increased response to a painful stimulus. It indirectly indicates central sensitization resulting from increased responsiveness, a decreased threshold, or changes in the receptive field (Sandkühler, 2009; Latremoliere and Woolf, 2009; Treede, 2012). Quantitative sensory testing (QST) (Rolke et al., 2006) and electrical QST (Vaneker et al., 2005) may be used to demonstrate hyperalgesia by longitudinal measurements of thresholds. To study the underlying nociceptive system, one may use low-intensity electrocutaneous stimulation with intra-epidermal needle electrodes, since it was shown to recruit nociceptive $\mathrm{A} \delta$-fibers preferentially, while bypassing mechanoreceptors (Inui et al., 2002; Mouraux et al., 2010; Steenbergen et al., 2012). Because of the low amplitudes, thresholds can only be determined from a sensory detection task rather than from a pain detection task.

Currently, there are few computational models of the nociceptive system (Britton and Skevington, 1989; Britton et al., 1996; Xu et al., 2008b; Farajidavar et al., 2008), but these focus on different stimulus modalities, i.e. thermal and tactile, and have a different outcome, i.e. pain sensation. As they do not include any stochastic subsystem, they cannot simulate trial-to-trial variability. Hence, there is no neurophysiologically plausible model for a detection task with electrocutaneous stimuli. Detection tasks yield binary responses (yes/no). In general, this involves a two-alternative forced choice task which can be modeled with a drift-diffusion model (DDM) that accumulates noisy sensory evidence until a decision threshold is reached (Ratcliff and Rouder, 1998; Bogacz et al., 2006; Ratcliff and McKoon, 2008). The DDM may be interpreted as a stochastically spiking neuron model with a spike 
corresponding to the detection of the stimulus. Here we consider a detection task with electrocutaneous stimulation (Doll et al., 2014), where subjects only report the detected stimuli. The DDM also yields reaction times, but, as they are not recorded in the experiment, this is less relevant.

The electrical stimulus is a square-wave pulse train characterized by four parameters, i.e. the amplitude $(A)$ and three temporal stimulus properties: the pulse width $(P W)$, the number of pulses $(N o P)$ and the inter-pulse interval $(I P I)$, see also Fig. 2.2. The detection threshold is the amplitude at which half of the stimuli are detected (Treutwein, 1995). This threshold was shown to depend on temporal stimulus properties for various related stimulus modalities. The strength-duration curve describes the relationship between the stimulus amplitude and its pulse width to activate a neuron (Lapicque, 1907; Mogyoros et al., 1996; Irnich, 2010). As NoP increases, the threshold for first sensation of vibrotactile stimuli decreases (Nunziata et al., 1989). Gescheider et al. (1999) found that the decrease in the detection threshold of vibrotactile stimuli when decreasing IPI was due to superposition of neural responses. Other studies suggest that with multiple pulses, the afferent input to secondary neurons is increased by temporal summation (van der Heide et al., 2009; Mouraux et al., 2014). However, this effect should wear off for large IPI and then the subject may detect both pulses independently (Zwislocki, 1960; Viemeister and Wakefield, 1991). This still increases the probability of detection. Hence, for a stimulus consisting of two pulses, a lower detection threshold is expected. However, the presence of temporal summation in the sensory detection task using nociceptive electrocutaneous stimuli has not been studied varying each single temporal property.

The aim of this study is to develop a computational model representing the essential peripheral and central mechanisms of processing of electrocutaneous stimuli. We want to replicate the experimental effects of all temporal properties on detection thresholds within this model. To facilitate parameter estimation, the model should be computationally efficient and have as few parameters as possible. We take the drift-diffusion model as a starting point for trial-to-trial variability in psychophysical experiments. Although widely applied, a disadvantage of this model is that it is analytically intractable, especially for time-dependent input. The alternative is to use simulations, which is time-consuming. We follow an approach by Plesser and Gerstner (2000) to replace the stochastic problem by a probabilistic hazard model through an escape process. This leads to an efficient model for a detection task without reaction times. 
As a motivation for the modeling, we first present preliminary experimental data from a human subject study. Next, we describe how electrical stimulation induces neural activity and leads to psychophysical responses. For the modeling, we incorporate peripheral fiber activation and sensory inputs at secondary neurons giving a drift-diffusion model. The activity can be close to threshold and this is different from the original hazard model. We propose a different hazard function and show that our hazard model fits nicely to the drift-diffusion model with respect to the psychophysical functions. Next we determine detection thresholds in the model and relate these to the experimentally observed thresholds. We discuss how the temporal properties affect detection thresholds based on the model and conclude with further applications of the hazard model.

\subsection{Psychophysical human-subject experiment}

For illustrative purposes, we present data from a psychophysical human-subject study with a yes-no detection task using electrocutaneous stimulation. The experiment considered in the present work is part of a more extended experiment. The psychophysical data and analysis in this chapter illustrates the effects of temporal properties on the detection task. The methodology and results of this human subject study is reported in an other work (Doll et al., 2015a).

\subsubsection{Methods}

Fifteen healthy human subjects participated in this study. The Medical Ethics Committee Twente approved all experimental procedures. All subjects provided written informed consent and were rewarded with a gift voucher after their participation in the experiment. Subjects visited the lab on two consecutive days. Experiments were conducted under the same conditions on each day. Electrical stimuli consisted of cathodic square-wave current pulses using an intra-epidermal needle electrode that was attached to the left forearm (Steenbergen et al., 2012; Doll et al., 2014). The electrical stimulus is characterized by the amplitude and three temporal properties: the number of pulses, the interpulse interval and the pulse width. The experimental procedure lasted for ten minutes. Stimuli were selected according to an adaptive probing procedure (Doll et al., 2014). Subjects were instructed to press and hold a response button until a stimulus was detected. After the release, they 
were instructed to re-press the button after about a second. The inter-onset interval between two consecutively applied stimuli varied from 2 to 5 seconds. Stimuli with four combinations of temporal properties, see Table 2.1 were presented in a pseudorandom order, but with an equal number of trials for each combination of temporal stimulus properties. Logistic regression was used to obtain a detection threshold

Table 2.1: Four combinations of temporal stimulus properties for the electrocutaneous pulse train stimulus. If $N o P=1$, then $I P I$ is undefined.

\begin{tabular}{ccccc}
\hline Index & 1 & 2 & 3 & 4 \\
\hline$N o P[\#]$ & 1 & 1 & 2 & 2 \\
$I P I[\mathrm{~ms}]$ & - & - & 10 & 50 \\
$P W[\mathrm{~ms}]$ & 0.42 & 0.84 & 0.42 & 0.42 \\
\hline
\end{tabular}

estimate from stimulus-response pairs per subject per day per combination of temporal properties. A two-way repeated measures ANOVA was used to study the effect of parameter combination and the effect of study day on the detection threshold. Mauchly's test was used to check violations of the sphericity assumption. Post-hoc comparisons were performed without correcting for possible type I errors, as the statistical analysis here is only meant to demonstrate the experimental phenomena.

\subsubsection{Results}

Two subjects were removed from the dataset due to technical issues on the second study day. The detection thresholds from individual subjects and the group are shown in Fig. 2.1. Mauchly's test indicated that the assumption of sphericity for parameter combination had been violated $\left(\chi^{2}(5)=.350, p=.047\right)$. Therefore, the degrees of freedom were corrected as using the Greenhouse-Geisser estimates of sphericity $(\epsilon=.614)$. The results show that there was a significant effect for parameter combination $(F(1.84,22.10)=66.82, p<.001)$. Study day had no significant effect on the detection thresholds $(F(1,12)=.19, p=.67)$. Post-hoc comparisons showed that increasing the pulse-width (i.e. comparison between combination 1 and 2 ) or the number of pulses (i.e. comparisons between combinations 1 and 3, 1 and 4, 2 and 3 , and between 2 and 4 ) significantly reduced the threshold ( $p \leq .001)$. The difference in thresholds between the two two-pulse combinations 3 and 4 was at the 


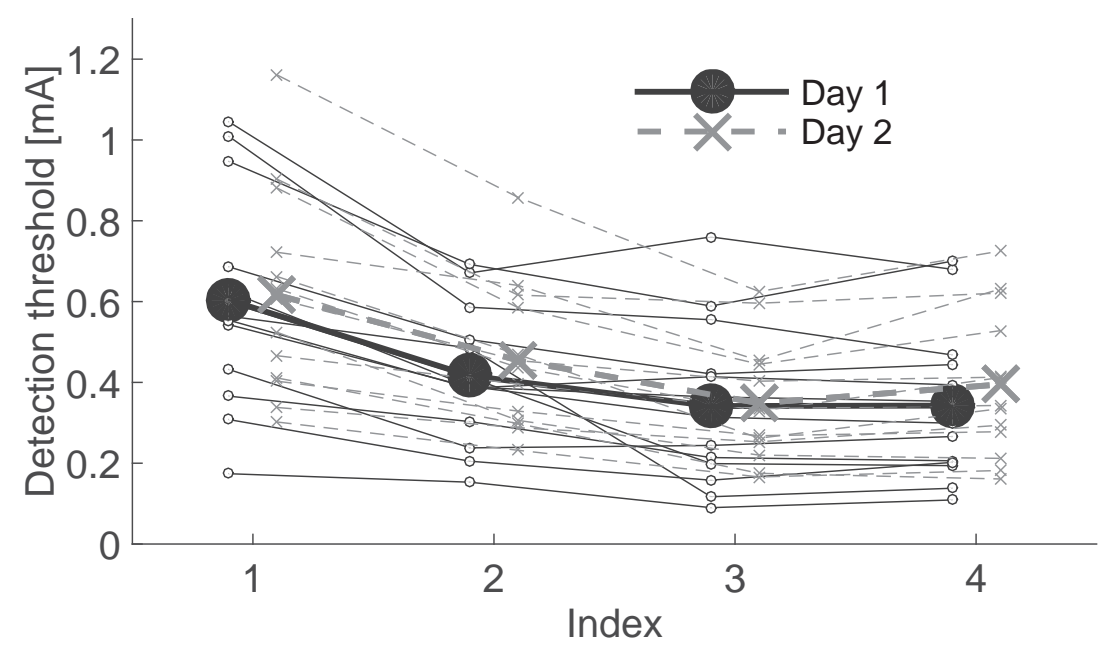

Figure 2.1: Detection thresholds for each subject, study day, and combination of temporal stimulus properties (Table 2.1). The larger solid circles and crosses present the mean detection thresholds for day 1 and day 2 , respectively.

significance level $(p=.051)$.

\subsection{Computational modeling}

Application of electrocutaneous stimulation charges nerve endings of $\mathrm{A} \delta$-fibers. Action potentials are generated given sufficient stimulation. When this neuronal activity reaches the synapses that project to neurons in the dorsal horn, this triggers the release of neurotransmitter from the pre-synaptic terminal, inducing an excitatory post-synaptic current (EPSC). Consequently, the membrane potential of post-synaptic neurons increases and ultimately an action potential is generated. Sufficient neuronal activity leads to a supraspinal response where a subject responds 'yes'. Otherwise, the subject did not detect the stimulus as the neuronal activity was not sufficiently high. To quantitatively describe this detection process, we model signal conduction from the skin to supraspinal sites. First, we formulate the dynamic process in a single signal channel. Each signal channel consists of nociceptors, a synapse and a secondary neuron. Second, for the trial-to-trial variability, we include small background noise as additional input for secondary neurons. We also propose a convenient alternative based on escape noise (Plesser and Gerstner, 2000). Lastly, we derive lumped models for the ascending nociceptive pathway by taking multiple signal channels into account. The organization of the neuronal system is sketched 
in Fig. 2.2 with multiple signal channels.

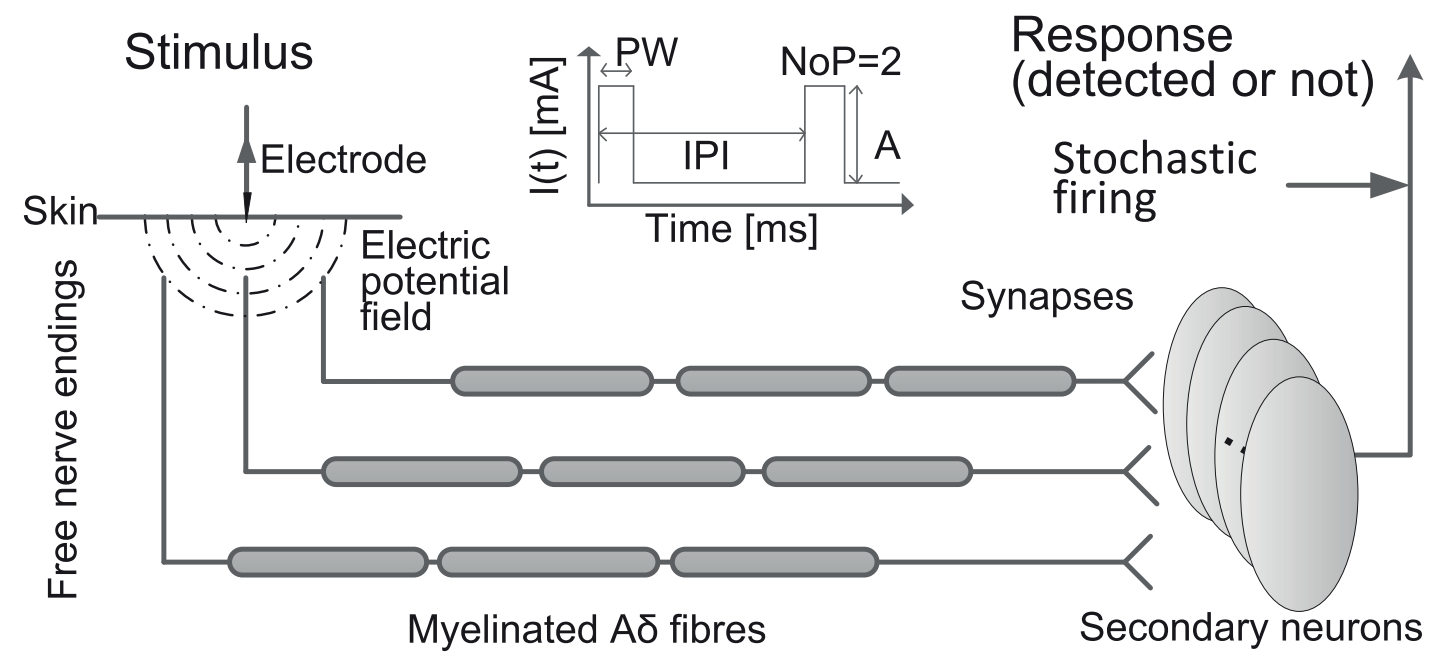

Figure 2.2: An electrode is attached to the skin of a subject to deliver pulse train stimulation. The dot-dashed concentric half circles represent the electric potential. Charging the nerve endings leads to traveling action potentials in the $\mathrm{A} \delta$-fiber. The arrival of spikes at the pre-synaptic terminal triggers the release of the glutamate from the synapse resulting in an EPSC. The secondary neuron is charged and the activity will converge upto the supraspinal part and lead to a binary response. Note that the number of signal channels is the number of secondary neurons, i.e. four in this diagram.

\subsubsection{Activation of afferent fibers}

For simplicity, we assume that the skin is a homogeneous medium with conductivity $c_{0}$, and the needle electrode is an infinitesimal point source generating an electric potential $V_{e}$. Hence, applying electrocutaneous stimulation with a constant current amplitude $A$, the electric potential is given by $V_{e}(r)=\frac{A}{4 \pi c_{0} r}$, where $r$ is the distance from the needle electrode. This electric potential generates the induced input to the $\mathrm{A} \delta$-fibers. Usually, the effective input is the second spatial derivative of the potential along a fiber (Rattay, 1999). However, in our experimental setup, relatively low amplitudes are applied, similar to (Mouraux et al., 2010). As a result, only the afferent fibers near the skin are recruited. In addition, the afferent fibers terminate in this region, and mostly with the nerve endings perpendicular to the skin. For these nerve endings, the effective input at distance $r$ is given by the first spatial derivative of the potential $I_{A}(r, t):=\frac{1}{c_{1}} \frac{\partial V_{e}}{\partial r}=-\frac{I(t)}{4 \pi c_{0} c_{1} r^{2}}$, where $c_{1}$ describe the resistance of nerve endings per unit length. For simplicity, we denote $c:=c_{0} c_{1}$. 
We use a cathodic electrode, so that the generated current $I(t)$ is always negative, and the induced input depolarizes the membrane of nerve endings. In the sequel, we will write $A$ instead of $|A|$. For simplicity, we take the nerve ending as a point in the three-dimensional space. Next, we model the dynamics of the membrane potential of the ending $V_{1}$ as a leaky integrator

$$
C_{1} \dot{V}_{1}=-G_{1} V_{1}+I_{A}(r, t), \quad V_{1}(r, 0)=0,
$$

where $C_{1}$ is the electrical capacitance of the nerve ending, and $G_{1}$ is the electrical conductance of the nerve ending. If $V_{1}$ exceeds a threshold $V_{t h}$, the fiber spikes. Given a single pulse stimulus with duration $P W$, the maximal potential of $V_{1}(r, t)$ is a function of the distance

$$
V_{m}(r):=\max _{t \in[0, T]} V_{1}(r, t)
$$

where $T$ is the interval of a single trial. For stimulation with a single square pulse, we have $V_{m}(r)=\frac{G_{1}^{-1} A}{4 \pi c r^{2}}\left(1-\exp \left(-\frac{P W}{C_{1} G_{1}^{-1}}\right)\right)$ based on $(2.1)$.

As the distance increases, the induced input decreases. So, the threshold $V_{t h}$ results in a critical value for the distance: all endings with a distance larger than this critical value are not activated. This critical value $r_{c}$ is computed by solving the equality $V_{m}\left(r_{c}\right)=V_{t h}$ :

$$
r_{c}=\left(\frac{G_{1}^{-1} A}{4 \pi c V_{t h}}\left(1-\exp \left(-\frac{P W}{C_{1} G_{1}^{-1}}\right)\right)\right)^{\frac{1}{2}} .
$$

So given the distance of a single nerve ending to the needle electrode, we can determine whether this ending generates a spike. Next, spikes from activated fibers drive the secondary neuron. We ignore the differences in the moments of action potential generation and also the arrival times of spikes at the secondary neuron. To describe the total input we need to determine how many nerve endings are recruited. We assume that there is a homogeneous density $\rho$ of nerve endings under the stimulated tissue beneath the electrode and a lower bound on the depth $h$ of the nerve endings from the skin, see Fig. 2.3. The number of the recruited endings $N_{r}$ is approximated to be proportional to the area of a circle within a sphere of radius $r_{c}$ at depth $h$ and is given by

$$
N_{r}=\pi \rho\left(r_{c}^{2}-h^{2}\right) H\left(r_{c}-h\right),
$$

where $H$ is a Heaviside step function: $H(x)=1$, when $x \geq 0 ; H(x)=0$, when $x<0$. Here we approximate the actually integer number of recruited endings by 
a continuous quantity. If $N_{r}$ is small, this may be unsatisfactory. We discuss this later, but for a more elaborate modelling study on this issue, we refer to Mørch et al. (2011).

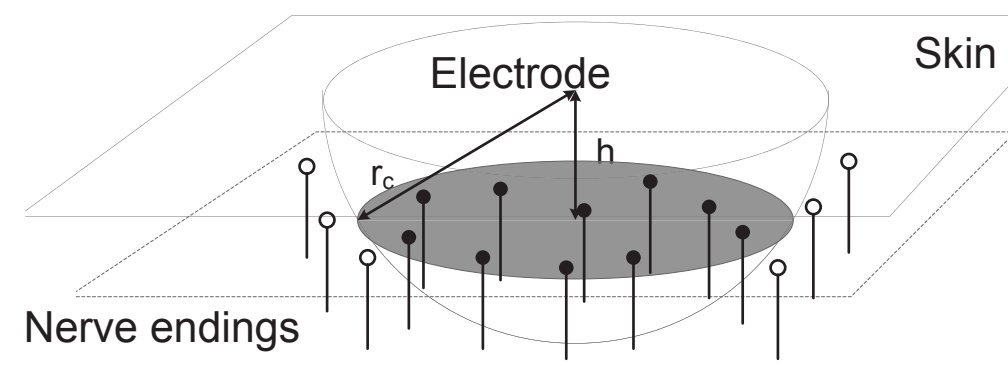

Figure 2.3: Illustration of the geometry of nerve endings under skin, a minimal depth is denoted by $h$. The endings with solid tips are recruited and those with empty tips are not recruited. The grey surface represents the recruited space, i.e. within critical distance $r_{c}$.

\subsubsection{Post-synaptic dynamics}

We describe the post-synaptic potential (PSP) $V_{2}(t)$ of a secondary neuron also as a leaky integrator

$$
C_{2} \dot{V}_{2}=-G_{2} V_{2}+I_{p}(t), \quad V_{2}(0)=0,
$$

where $C_{2}$ is the electrical capacitance of the secondary neuron, $G_{2}$ is the electrical conductance of the secondary neuron, and $I_{p}(t)$ is the EPSC. This EPSC is proportional to the potential gradient between the post-synaptic and AMPA-reversal potentials, $\left(V_{2}-E_{A M P A}\right)$. As the inter-stimulus interval in repetitive electrocutaneous stimulation varied from 2 to 5 seconds, it is justified to assume that synaptic plasticity did not occur between trials. The IPI used for double-pulse stimuli is in the order of tens of milliseconds. This might involve short-term synaptic facilitation or depression at synapses from afferent fibers onto dorsal horn neurons. As recently reviewed in (Luo et al., 2014), both may occur for various synapses, and the net effect is uncertain. Therefore, we do not include it here. Hence, we choose a simple reset-decay model for fast AMPA synapses (Roth and van Rossum, 2009), whose impulse response is $g(t)=\bar{g} \exp \left(-t / \tau_{s}\right)$ for $t \geq 0$ with decay constant $\tau_{s}=1.5 \mathrm{~ms}$ and maximal conductance $\bar{g}$ as a constant (Gabbiani et al., 1994). It is justified to set $V_{2}-E_{A M P A} \approx V_{R}-E_{A M P A}$ to some constant $K$, as we consider $V_{2}$ only below but close to the firing threshold $V_{R}$. The more afferent fibers are activated, 
the more pre-synaptic spikes are expected. To determine the precise timing of presynaptic spikes, both spike propagation and the variability of conduction velocity might play a role. First, the myelinated $\mathrm{A} \delta$ fibers permit generated spikes recruited by relatively low stimulation frequency at nerve endings propagate along the nerves robustly. Second, the variability of conduction velocities of $\mathrm{A} \delta$ fibers could lead to the variability in the arrival times at pre-synaptic terminals. However, the variability of conduction velocity for fibers from the same area is expected to be small. With a typical value of the conduction velocity for the $\mathrm{A} \delta$ fiber $20 \mathrm{~m} / \mathrm{s}$ and a distance of $50 \mathrm{~cm}$, the spread of compound pre-synaptic spikes at the dorsal horn is expected to at most a few milliseconds. To determine post-synaptic activity, we do not take the variability of the conduction variability or the arriving times into account as the secondary neuron has a much larger time constant (Weng et al., 2006). These considerations encourage us to simplify pre-synaptic spikes from the activated afferent fibers by

$$
u(t)=N_{r} \sum_{k=0}^{N o P-1} \delta(t-k I P I)
$$

with $\delta$ the Dirac delta function. Its convolution with $K g(t)$ gives the $\operatorname{EPSC} I_{p}(t)$ :

$$
\begin{aligned}
I_{p}(t) & :=(K g * u)(t)=\int_{0}^{\infty} K g(\tau) u(t-\tau) d \tau \\
& =\frac{N_{r} \tau_{s} \bar{g} K}{\tau_{s}} \sum_{k=0}^{N o P-1} \exp \left(-\frac{t-k I P I}{\tau_{s}}\right) H(t-k I P I)
\end{aligned}
$$

Note that $N_{r} \tau_{s} \bar{g} K$ is a factor from afferent fibers, synapses and secondary neurons; the remaining $\tau_{s}$-normalized term facilitates the computation of $V_{2}$ by its convolution with the transfer function of the cascaded leaky integrator (2.5).

\subsubsection{Stimulus detection by randomly spiking secondary neu- rons}

The activity evoked in afferent fibers induces post-synaptic activity in secondary neurons of the dorsal horn. This synaptic activity is noisy so that secondary neurons spike stochastically. We consider two descriptions of this random behavior: one stochastic and one probabilistic. We assume that a stimulus is detected if at least one secondary neuron spikes. 


\subsubsection{Stochastic description: a drift-diffusion model}

To describe the noisy dynamics of $V_{2}$, we employ the drift-diffusion model (Ratcliff and McKoon, 2008). In contrast to the stimulation-induced pre-synaptic pulses, background pre-synaptic pulses are relatively weak. Assuming a large number of background pulses impinges on the neurons per membrane time constant, the net input to post-synaptic neurons can be modeled as additive white noise (Capocelli and Ricciardi, 1971). Hence, the model (2.5) becomes a stochastic differential equation $(\mathrm{SDE})$ with a deterministic term $I_{p}(t)$ and white noise input

$$
C_{2} d V_{2}=\left(-G_{2} V_{2}+I_{p}(t)\right) d t+\sigma_{\xi} d W, \quad V_{2}(0)=0
$$

where $\sigma_{\xi}$ is the noise strength and $W$ is a standard Wiener process. We describe the binary outcome of 'spiking or not' of a single secondary neuron by

$$
R_{s}:=H\left(\max _{t \in[0, T]} V_{2}(t)-V_{R}\right),
$$

where we fix the trial interval $T=500 \mathrm{~ms}$. When $R_{s}=1$, it means that the neuron generated at least one spike within the trial interval $T$, otherwise none. We use the Euler-Maruyama scheme to obtain a single realization of the DDM with a fixed timestep of $0.01 \mathrm{~ms}$ (Kloeden and Pearson, 1977). We approximate the probability of at least one spike $\Psi_{D, s}$ by the average of $N=200$ realizations

$$
\Psi_{D, s}:=\operatorname{Pr}(\text { spike })=\overline{R_{s}} \approx \frac{1}{N} \sum_{i=1}^{N} R_{s, i} .
$$

At the level of the spinal cord, there are multiple secondary neurons that receive the stimulus induced input. We assume this input is identical, but that the noise is independent. Then, for a population with $l$ signal channels, the probability that at least one spike occurs, is given by

$$
\Psi_{D}:=1-\left(1-\Psi_{D, s}\right)^{l} .
$$

Note that this also defines the corresponding psychophysical curve for the DDM.

\subsubsection{Probabilistic description: a hazard model}

Escape noise (Plesser and Gerstner, 2000) is another way to describe random spiking, given noise-free dynamics of secondary neuron (2.5) and (2.7). In other 
words, at each moment, the neuronal activity could exceed the firing threshold with a certain probability, even if the deterministic activity is below the firing threshold. We describe this stochastic firing with a non-homogeneous Poisson process. For the time-varying firing rate of the Poisson process we must choose a hazard function $\lambda_{s}$, which depends on the noise-free PSP. We take the widely used sigmoidal activation function for the hazard function

$$
\lambda_{s}(t):=\lambda_{s}\left(V_{2}(t)\right)=\frac{\lambda_{h}}{1+\exp \left(-\left(V_{2}(t)-\alpha_{h}\right) / \sigma_{h}\right)},
$$

where $\alpha_{h}$ is the activation threshold, $\lambda_{h}$ is the maximal firing rate and $\sigma_{h}$ is the slope parameter. Note that the activation threshold $\alpha_{h}$ has a different interpretation from the firing threshold $V_{R}$ in the DDM. In the DDM, given a realization of noise, the firing threshold determines the spiking in a deterministic way. In the hazard model, even if the noise-free PSP is below $\alpha_{h}$, there is still a probability to spike.

For a single neuron, the expected value of the number of spikes during this interval is given by

$$
\lambda_{T}^{s}:=\int_{0}^{T} \lambda_{s}(t) d t
$$

Thus, the probability of at least one spike in a single secondary neuron is given by

$$
\Psi_{H, s}:=1-\operatorname{Pr}(\text { no spike } \mid 0 \leq t \leq T)=1-\exp \left(-\lambda_{T}^{s}\right)
$$

For a population of neurons, similar to Eq. (2.11), we obtain the psychophysical function

$$
\Psi_{H}:=1-\left(1-\Psi_{H, s}\right)^{l}=1-\exp \left(-l \lambda_{T}^{s}\right)
$$

\subsubsection{Lumped models}

We have built two models to represent the stimulus processing from electrocutaneous stimulation to random binary responses. However, these models have more than ten unknown physical quantities. To reduce the number of parameters, we introduce six lumped parameters for each model.

If we let the time constant of secondary neurons $\tau_{2}:=C_{2} G_{2}^{-1}$, the lumped PSP $x:=G_{2} V_{2} / q$, the strength of white noise $\sigma:=\sigma_{\xi} / q$, the lumped EPSC $I_{p}^{*}:=I_{p} / q$ and the scaled firing threshold $\alpha_{2}:=G_{2} V_{R} / q$ where $q$ is an arbitrary but non-zero constant, then the SDE can be rewritten as

$$
\tau_{2} d x=\left(-x+I_{p}^{*}(t)\right) d t+\sigma d W, \quad x(0)=0 .
$$


For a single neuron the binary response is given by $R_{s}=H\left(\max _{t \in[0, T]} x(t)-\alpha_{2}\right)$, from which we can derive the psychophysical curves using Eqs. (2.10) and (2.11).

The gain factors in peripheral activation, central processing, and synaptic transmission are given by $\kappa:=\rho\left(4 \pi c G_{1} V_{t h}\right)^{-1}, K$ and $\bar{g} \tau_{s}$, respectively. All those gain factors are independent of the dynamics in underlying mechanisms. Hence, lumping those factors into the factor $q:=\bar{g} \tau_{s} \kappa K$, also see (2.7), we meet the requirement to get as few parameters as possible. Denoting the time constant of afferent fibers $\tau_{1}:=C_{1} G_{1}^{-1}$, we can write $N_{r}=\kappa\left[f_{A}-\alpha_{1}\right]_{+}$, where $[z]_{+}:=\pi z H(z)$ is a threshold-linear function, $\alpha_{1}:=4 \pi c G_{1} V_{t h} h^{2}$ is the lumped activation threshold, and $f_{A}:=A\left(1-\exp \left(-\frac{P W}{\tau_{1}}\right)\right)=4 \pi c G_{1} V_{t h} r_{c}^{2}$ is the amount of activation of afferent fibers.

Lumping the hazard model we introduce $\alpha_{1}, \tau_{1}, \tau_{2}$ as for the DDM, the lumped activation threshold of secondary neurons $\alpha_{L}:=G_{2} \alpha_{h} / q$, the lumped slope parameter $\sigma_{L}:=G_{2} \sigma_{h} / q$ and the population firing rate $\lambda_{L}:=l \lambda_{h}$. It is now straightforward to compute the psychophysical function using the scaled noise-free dynamics $x^{0}(t):=G_{2} V_{2}(t) / q$

$$
\begin{aligned}
x^{0}(t):= & \frac{\left[f_{A}-\alpha_{1}\right]_{+}}{\tau_{2}-\tau_{s}} \sum_{k=0}^{N o P-1}\left(\exp \left(-\frac{t-k I P I}{\tau_{2}}\right)\right. \\
& \left.-\exp \left(-\frac{t-k I P I}{\tau_{s}}\right)\right) H(t-k I P I)
\end{aligned}
$$

by evaluating the integral

$$
\Psi_{H}=1-\exp \left(-\int_{0}^{T} \lambda(t) d t\right)
$$

where $\lambda(t)=\lambda_{L}\left(1+\exp \left(-\left(x^{0}(t)-\alpha_{L}\right) / \sigma_{L}\right)\right)^{-1}$.

To summarize, the lumped DDM involves six parameters: the threshold $\alpha_{1}$ and the time constant $\tau_{1}$ in the peripheral nervous system; the threshold $\alpha_{2}$, the noise strength $\sigma$, the time constant $\tau_{2}$ and the number of secondary neurons $l$ in the more central system. Note that the lumped parameters $\alpha_{2}$ and $\sigma$ combine properties of the peripheral and central subsystem, as they are scaled by $q$. For the lumped hazard model, we have the same $\alpha_{1}, \tau_{1}$ and $\tau_{2}$, but the other three $\alpha_{L}, \sigma_{L}$ and $\lambda_{L}$ have a different interpretation. We will write $\boldsymbol{\theta}_{D}:=\left(\alpha_{1}, \tau_{1}, \tau_{2}, \alpha_{2}, \sigma, l\right)$ and $\boldsymbol{\theta}_{H}:=\left(\alpha_{1}, \tau_{1}, \tau_{2}, \alpha_{L}, \sigma_{L}, \lambda_{L}\right)$ for the DDM and the HM, respectively. 


\subsubsection{Comparison of the dynamics and psychophysical func- tions of DDM and HM}

We formulated two models for the same detection task. These two models have the same fiber activation, but different formulations for spiking of secondary neurons. We present their dynamics and study how their psychophysical functions differ.

\subsubsection{Activation of afferent fibers}

Fixing $P W$, the activation of afferent fibers $\left[f_{A}-\alpha_{1}\right]_{+}$follows a thresholdlinearity about $A$. Fixing the amplitude, the activation of afferent fibers grows by increasing $P W$ saturating to rheobase. This is illustrated in Fig. 2.4 fixing parameter values $\alpha_{1}=0.2 \mathrm{~mA}, \tau_{1}=0.12 \mathrm{~ms}$ and either $\mathrm{A}: P W=0.42 \mathrm{~ms}$ or $\mathrm{B}: A=0.5 \mathrm{~mA}$.
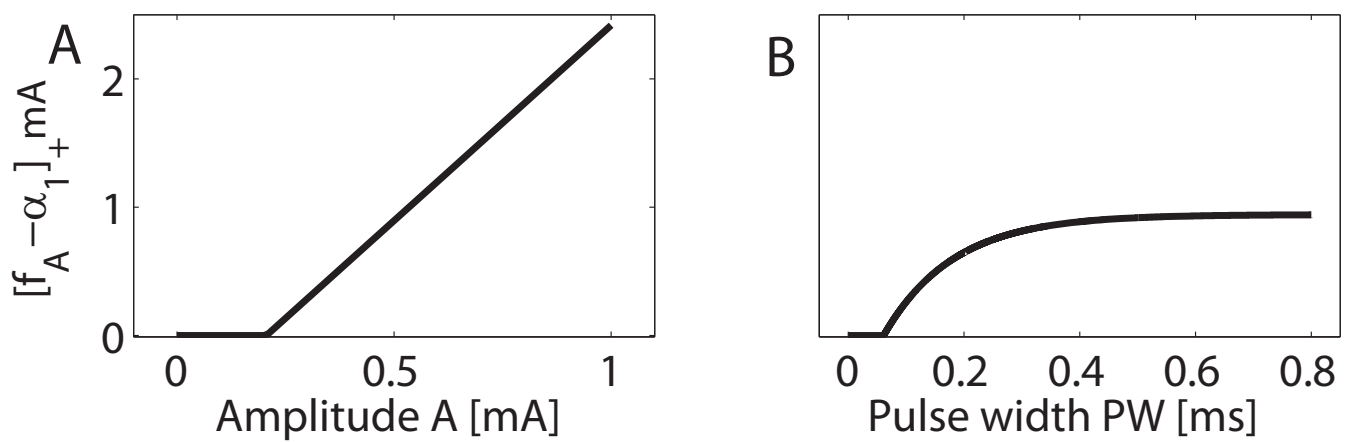

Figure 2.4: Activation of afferent fibers. A: activation has a threshold nonlinear relation with amplitude $A$; B: peripheral activity increases by increasing $P W$, eventually saturating.

\subsubsection{Dynamics of secondary neurons}

We set stimulus properties $A=1 \mathrm{~mA}, N o P=2, I P I=50 \mathrm{~ms}$, and $P W=$ $0.42 \mathrm{~ms}$ and system parameters $\alpha_{1}=0.5 \mathrm{~mA}, \tau_{1}=0.1 \mathrm{~ms}, \tau_{2}=50 \mathrm{~ms}, \sigma=$ $0.05 \mathrm{~A} / \mathrm{s}$ and $l=1$. The values of time constants $\tau_{1}$ and $\tau_{2}$ are based on (Mogyoros et al., 1996; Weng et al., 2006). In Fig. 2.5 we show realizations with and without noise in the DDM. Next, to demonstrate the dynamics of the HM, we use the same parameter values but for the parameters associated with secondary neurons we use $\alpha_{L}=0.01 \mathrm{~A} / \mathrm{s}, \sigma_{L}=0.001 \mathrm{~A} / \mathrm{s}$ and $\lambda_{L}=0.01 \mathrm{kHz}$ using three different stimuli with the same $P W=0.42 \mathrm{~ms}: N o P=1$ (thick dashed); $N o P=2$ and $I P I=50 \mathrm{~ms}$ (solid); $N o P=2$ and $I P I=150 \mathrm{~ms}$ (dot-dashed). The dynamics and the expected 

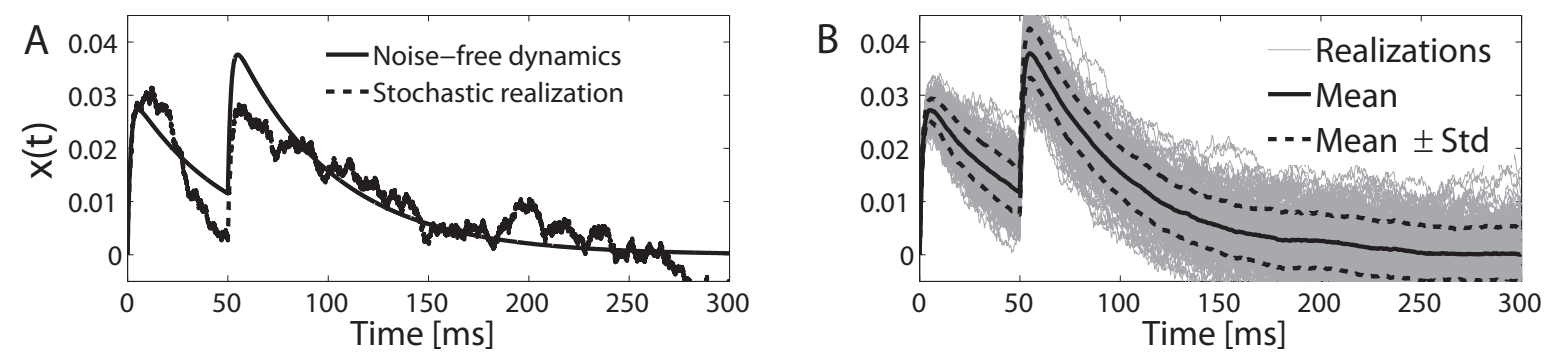

Figure 2.5: Stochastic dynamics of the DDM using a pulse train current input with ( $A=1 \mathrm{~mA}, N_{o} P=2, I P I=50 \mathrm{~ms}, P W=0.42 \mathrm{~ms}$ ). A: noise-free dynamics (solid) with $\sigma=0$ and a stochastic realization (dashed). B: $N=200$ realizations of the stochastic dynamics (thin solid). Statistics of the potential are also shown, mean (solid), and mean plus or minus the standard deviation (dashed). See text for system parameter values.

firing rate $\lambda_{T}$ are shown in Fig. 2.6. As the trial interval $T$ is much larger than the time constant $\tau_{2}$, the psychophysical function value $\Psi_{H}(A)$ does not change for larger values of $T$. We implemented both models in MATLAB R2010b on
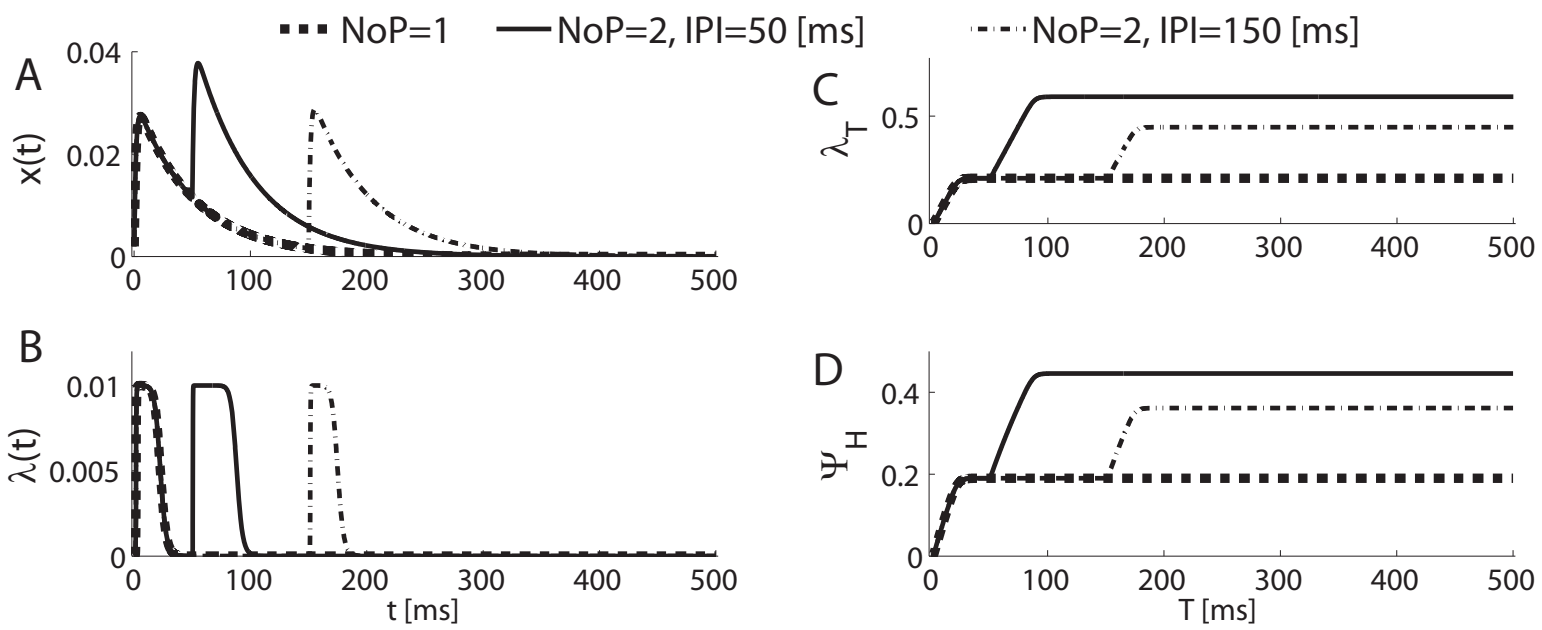

Figure 2.6: Activities of secondary neurons using three different stimuli with the same $P W=0.42 \mathrm{~ms}: N o P=1$ (thick dashed); $N o P=2$ and $I P I=50 \mathrm{~ms}$ (solid); $N o P=2$ and $I P I=150 \mathrm{~ms}$ (dotted-dashed). A: Lumped PSP stimulated by an electrical train of two pulses with amplitude $A=1 \mathrm{~mA}, I P I=50 \mathrm{~ms}$ and $P W=0.42 \mathrm{~ms}$; B: instantaneous firing rate; $\mathrm{C}$ : the expected value of the number of spikes within a trial $[0, T]$; D: psychophysical function value $\Psi_{H}$ depending on $T$.

a desktop with an Intel Core i7 processor. The time needed to evaluate a single psychophysical function value $\Psi(A=0.1)$ was 0.21 second for the DDM using 4 cores and 0.0088 second for the HM. Hence, the HM is computationally much cheaper than 
the DDM.

\subsubsection{Comparing psychophysical functions of DDM and HM}

Since the psychophysical function of the HM is smooth, we start by choosing parameters that lead to experimentally plausible psychophysical functions for the DDM. Next we fit the psychophysical function of the HM to the DDM at discrete stimulus amplitudes. The parameters $\tau_{1}, \tau_{2}$ and $\alpha_{1}$ are the same for both models and hence we will use the same values for the DDM and the HM. We do this for several combinations of the temporal properties, see Table 2.2.

Table 2.2: Combinations of the temporal stimulus properties.

\begin{tabular}{lllllllll}
\hline Index & $\mathrm{A}$ & $\mathrm{B}$ & $\mathrm{C}$ & $\mathrm{D}$ & $\mathrm{E}$ & $\mathrm{F}$ & $\mathrm{G}$ & $\mathrm{H}$ \\
\hline NoP \# & 1 & 1 & 1 & 2 & 2 & 2 & 2 & 2 \\
$I P I[\mathrm{~ms}]$ & - & - & - & 10 & 20 & 50 & 100 & 150 \\
$P W[\mathrm{~ms}]$ & 0.21 & 0.42 & 0.84 & 0.42 & 0.42 & 0.42 & 0.42 & 0.42 \\
\hline
\end{tabular}

We use the relative fitting error to assess the difference between the HM and the DDM

$$
E=\sum_{j} \frac{\sum_{i}\left(\Psi_{D, j}\left(A_{i}\right)-\Psi_{H, j}\left(A_{i}\right)\right)^{2}}{\sum_{i} \Psi_{D, j}\left(A_{i}\right)^{2}},
$$

where $i$ is the index of amplitudes, $A_{i}$ ranges from 0 to 2 with a step $0.01 \mathrm{~mA}, j$ is the index of the combination of temporal properties, $\Psi_{D}$ means the psychophysical function based on the DDM, and $\Psi_{H}$ is the psychophysical function based on the HM. We minimize the error term by tuning values of three parameters $\alpha_{L}, \sigma_{L}$, and $\lambda_{L}$. We implement the minimization procedure in MATLAB 2010b by using the LSQNONLIN routine.

For a particular choice of parameter values, the psychophysical functions after fitting are shown in Fig. 2.7. The realizations of the binary responses for exactly the same amplitude follow a binomial distribution. Hence, we compute the confidence interval (CI) using the Clopper-Pearson method (Clopper and Pearson, 1934). For stimulus combinations D and $\mathrm{H}$, the psychophysical curves of the fitted HM lie within the $95 \%$ CI of $\Psi_{D}$. For other combinations, the fitted $\Psi_{H}$ deviates negligibly from $\Psi_{D}$, in particular for amplitudes far below or above the detection threshold.

We also study the fitting performance over a larger range of parameters for 


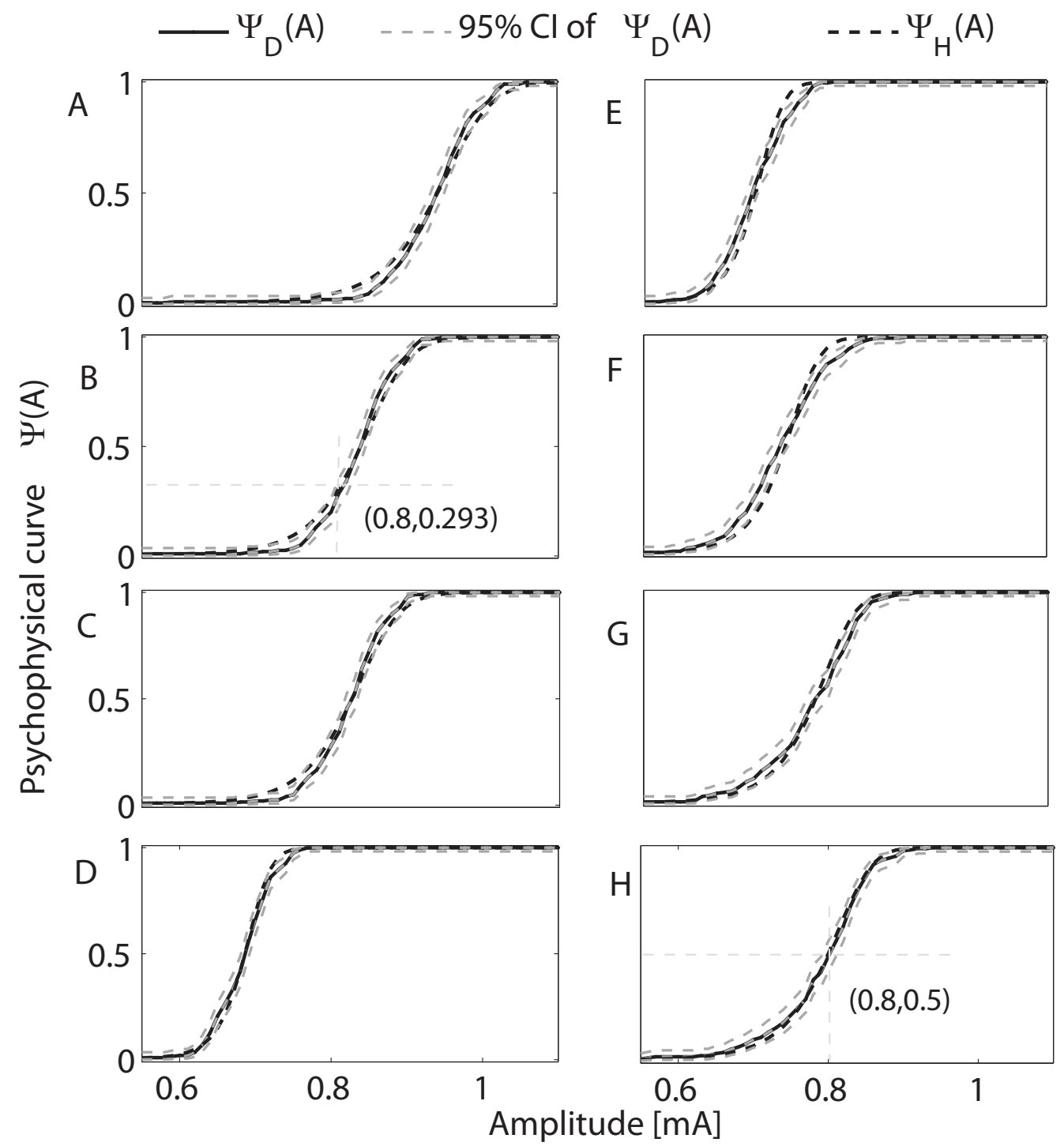

Figure 2.7: Using 8 different combinations of temporal stimulus properties (Temporal properties used in panels A-H correspond to combinations A-H in Table 2.2), psychophysical function values $\Psi_{D}(A)$ using the DDM (solid lines) and best fitted $\Psi_{H}$ by the HM (dashed lines) with fitting error $E=0.0029$. The common parameters are set to $\alpha_{1}=0.5 \mathrm{~mA}$, $\tau_{1}=0.1 \mathrm{~ms}, \tau_{2}=50 \mathrm{~ms}$, and $\tau_{s}=1.5 \mathrm{~ms}$. The parameters corresponding to neuronal variability are different in these two models. In the diffusion model, we set the values of parameter as $\alpha_{2}=0.02 \mathrm{~A} / \mathrm{s}, \sigma=0.05 \mathrm{~A} / \mathrm{s}$, and $l=1$, while the fitting results in $\alpha_{L}=0.0220 \mathrm{~A} / \mathrm{s}, \sigma_{L}=0.0021 \mathrm{~A} / \mathrm{s}$, and $\lambda_{L}=0.4020 \mathrm{kHz}$. The asymptotic behavior of the detection threshold with two independent pulses and its relation to the psychophysical curve with $N_{o P}=1$ are illustrated by the thin dashed lines in panels B and $\mathrm{H}$.

the DDM. We set two restrictions on the choice of the parameter values. First, we set ranges for the lumped threshold parameters so that the model detection 
thresholds are in the range of experimental observations, see Table. 2.3. For the time constants, the range of $\tau_{1}$ is set according to (Mogyoros et al., 1996); The range of $\tau_{2}$ is $5-200 \mathrm{~ms}$ based on time constants of wide dynamic range neurons in rat dorsal horn (Weng et al., 2006). Second, as the electrode only delivers stimulation with low intensity, when $A=0$, the detection probability should be relatively low, i.e. near 0 ; when $A=2 \mathrm{~mA}$ (the highest amplitude experimentally used), this probability should be close to 1 . Therefore, our second restriction is $\Psi_{D}(A=0)<0.35$ and $\Psi_{D}(A=2.0)>0.65$.

Table 2.3: Parameter space for the DDM. The upper three parameters are the same for the DDM and the HM.

\begin{tabular}{ccc}
\hline parameter & lower bound & upper bound \\
\hline$\alpha_{1}$ & 0.05 & 1.00 \\
$\tau_{1}$ & 0.01 & 0.50 \\
$\tau_{2}$ & 5 & 200 \\
$\alpha_{2}$ & 0.01 & 0.30 \\
$\sigma$ & 0.02 & 0.20 \\
$l \in \mathbb{Z}^{+}$ & 1 & 20 \\
\hline
\end{tabular}

With these restrictions, we apply a Monte Carlo method to study the fitting performance among the parameter space with the following steps. First, we sample a parameter vector $\boldsymbol{\theta}_{D}$ within the parameter space randomly. Next, we verify whether the sampled parameter vector satisfies the second restriction; if yes, we continue, otherwise, we discard this sample and redo the first step to sample another parameter vector. Then we compute $\Psi_{D}$, estimate parameters $\left(\alpha_{L}, \sigma_{L}, \lambda_{L}\right)$ for the HM and compute the fitting error $E$. We do these steps 500 times so that we obtain a set of errors. Finally, we determine the empirical distribution of the fitting error denoted by $F_{E}$, see Fig. 2.8. This plot describes how well the HM can be fitted to the DDM in the parameter space. The ideal result of fitting would be $E \equiv 0$ while, in practice, model differences cause differences between psychophysical functions. The goodness of fit can be assessed by looking at the error level when $F_{E}$ crossing $50 \%$, i.e. the level which half of fittings do not exceed. According to Fig. 2.8, we have $50 \%$ to have a fitting error $E \leq 0.040$. This result shows that psychophysical functions of the HM are similar to those generated by the DDM, for most choices of the parameters of the DDM. 


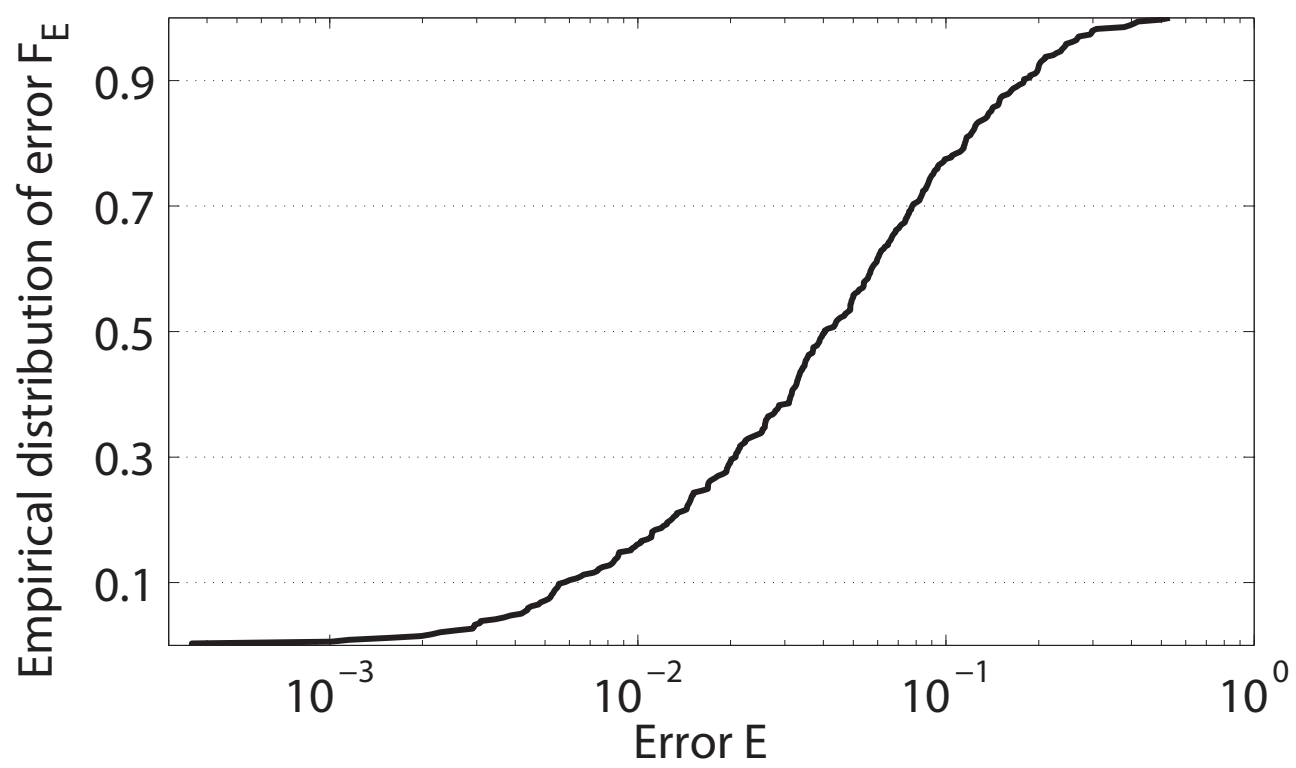

Figure 2.8: Distribution of the fitting error of the DDM by the hazard model with randomly chosen values of system parameters $\tau_{1}, \alpha_{1}, \tau_{2}, \alpha_{2}, \sigma$ and $l$ in the DDM.

\subsection{Effects of temporal stimulus properties on de- tection thresholds}

Detection thresholds are important psychophysical quantities and they depend on stimulus properties. We compare model-based thresholds with experimental values. We give a neurophysiological interpretation of the effects of temporal stimulus properties on detection thresholds using the two models.

We can determine the threshold $A_{50}$ in a model by solving $\Psi\left(A_{50}\right)=0.5$. This definition only makes sense if $\Psi(A=0)<0.5$, i.e. spontaneous activation is unlikely in the absence of stimuli. Therefore, for the HM, we impose the condition $T \lambda_{L}<\ln (2)\left(1+\exp \left(\alpha_{L} / \sigma_{L}\right)\right)$. If this is satisfied, it is straightforward to obtain the unique threshold as the psychophysical function is a monotone function of the stimulus amplitude in the hazard model. For the DDM it is nontrivial to derive such a condition as it would require to evaluate infinite-dimensional integrals for which no closed-form formula exists. Hence, for the DDM, we rely on (many) simulations to find $\Psi_{D}$ and determine $A_{50}$ by interpolation.

We now consider the experimentally observed detection thresholds using our models. Given a parameter set and the experimental combinations, for which we re- 
fer to Table 2.1, we can compute the detection thresholds of the models. Varying parameters systematically, we found parameter sets $\boldsymbol{\theta}_{D}=(0.06,0.4,50,0.031,0.09,8)$ and $\boldsymbol{\theta}_{H}=(0.06,0.4,50,0.006,0.001,0.01)$ such that detection thresholds of both models were close to the experimental values, see Fig. 2.9A. This illustrates that both models replicate the experimental phenomena, i.e. increasing $P W$ and $N o P$ decreases detection thresholds. From Fig. 2.4 we see that increasing $P W$ leads to more activated fibers. This increases the input to secondary neurons making them more likely to spike, hence decreasing the threshold. The models also explain why more pulses lower the detection threshold. For the HM, the activity $x^{0}$ after the first pulse returns to base-line if $I P I$ is large. Hence, for two independent pulses, the expected firing rate doubles, i.e. $\lambda_{T}\left(A, N_{O} P=2\right)=2 \lambda_{T}\left(A, N_{O} P=1\right)$, illustrated in Fig. 2.6C. So, we see $\Psi_{H}(A, N o P=2)=1-\left(1-\Psi_{H}(A, N o P=1)\right)^{2}>\Psi_{H}(A, N o P=1)$. The resulting $\Psi_{H}(N o P=2)$ is shifted to the left and steeper. This reflects that it is more likely to detect at least one of two independent stimuli. A similar reasoning holds for the DDM as a spike is more likely to occur, as two pulses increase the probability for the stochastic PSP to exceed the firing threshold. We can also use the latter relation to predict the threshold $A_{2,50}$ for two pulses with large IPI based on the psychophysical function of one pulse giving the equation $\Psi_{H}\left(A_{2,50}, N o P=1\right)=1-\frac{\sqrt{2}}{2}$. The detection thresholds $A_{2,50}$ computed from this relation are indicated in our comparison of DDM and HM in Fig. 2.7(B,H) and for the experimental data by the horizontal lines in Fig. 2.9A.

In addition, changing IPI from 10 to $50 \mathrm{~ms}$, we see that both experimental and model-based thresholds do not vary much. We have computed the thresholds for varying $I P I$ from 5 to $250 \mathrm{~ms}$, see Fig. 2.9B. This relation exhibits a value of IPI with minimal $A_{50}$. At this value, temporal summation of the PSP maximizes the expected value of the number of spikes for the hazard model. Likewise, for the drift-diffusion model it increases the time-window where the dynamics is just below threshold. Therefore, also for the DDM we find a value of IPI that minimizes $A_{50}$. Now for the data, the experimentally used IPI in Table 2.1 may have been either on both sides of such an optimal value or on the long flat tail, see the horizontal lines in Fig. 2.9A. In both cases, the models explain why the effect of IPI on threshold may be non-significant and at best small. The psychometric function describes the probability to detect stimuli at various amplitudes. To demonstrate the effect of temporal summation, one can fix the amplitude and compute this probability as a function of $I P I$. In Fig. 2.10, we show similar non-monotone trends of the detection probability as $I P I$ is increased for both models for the same parameter 

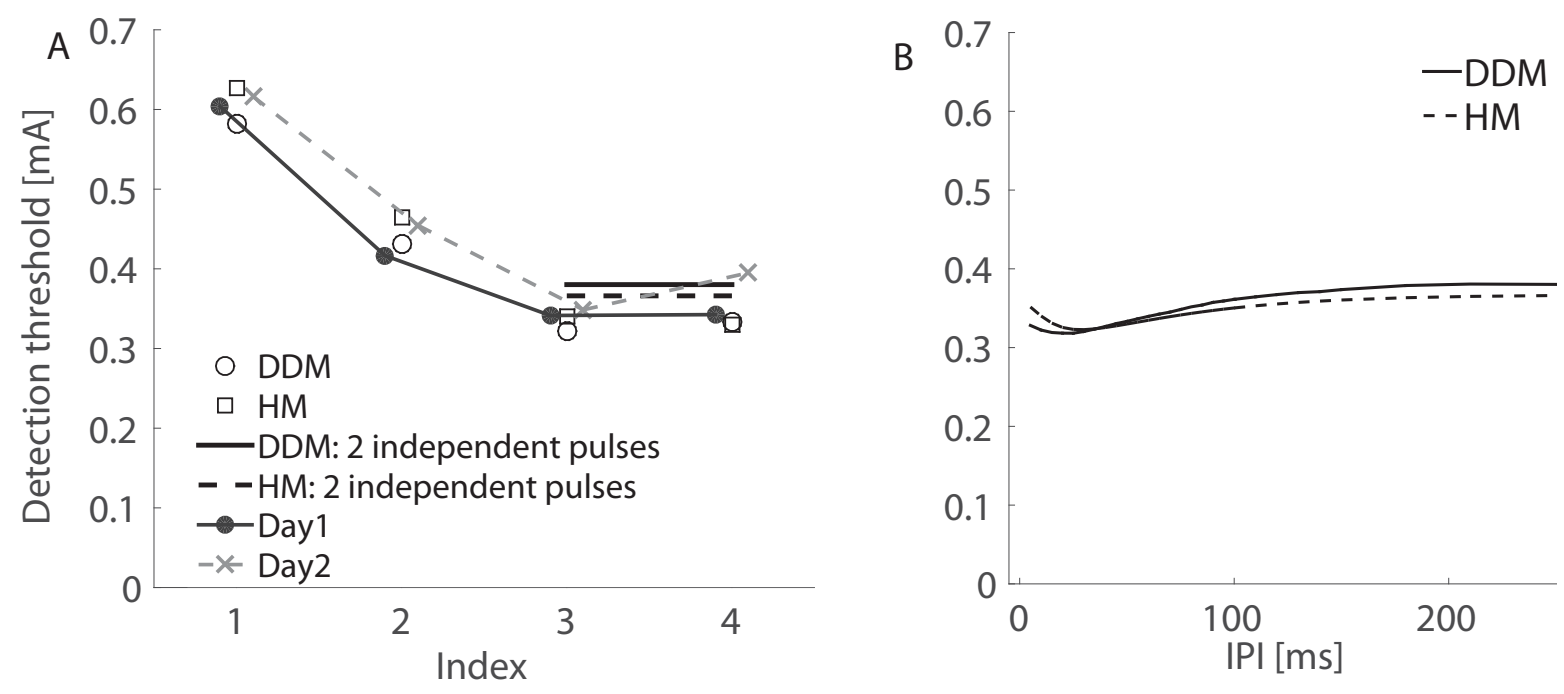

Figure 2.9: Dependence of detection thresholds on temporal properties. A: Experimental detection thresholds (solid circles and crosses) as in Fig. 2.1 and model-based thresholds (DDM: open circles, HM: open squares). The index refers to the combinations of Table 2.1 and see text for parameter values. The horizontal lines for $N o P=2$ indicate the asymptotic value $A_{2,50}$ for two independent pulses (DDM: solid line, HM: dashed line). B: non-monotone dependence of two-pulse threshold $A_{2,50}$ on IPI.

values. These simulated trends are in line with the $I P I$ effect in an eyeblink response task (Blumenthal et al., 1987).

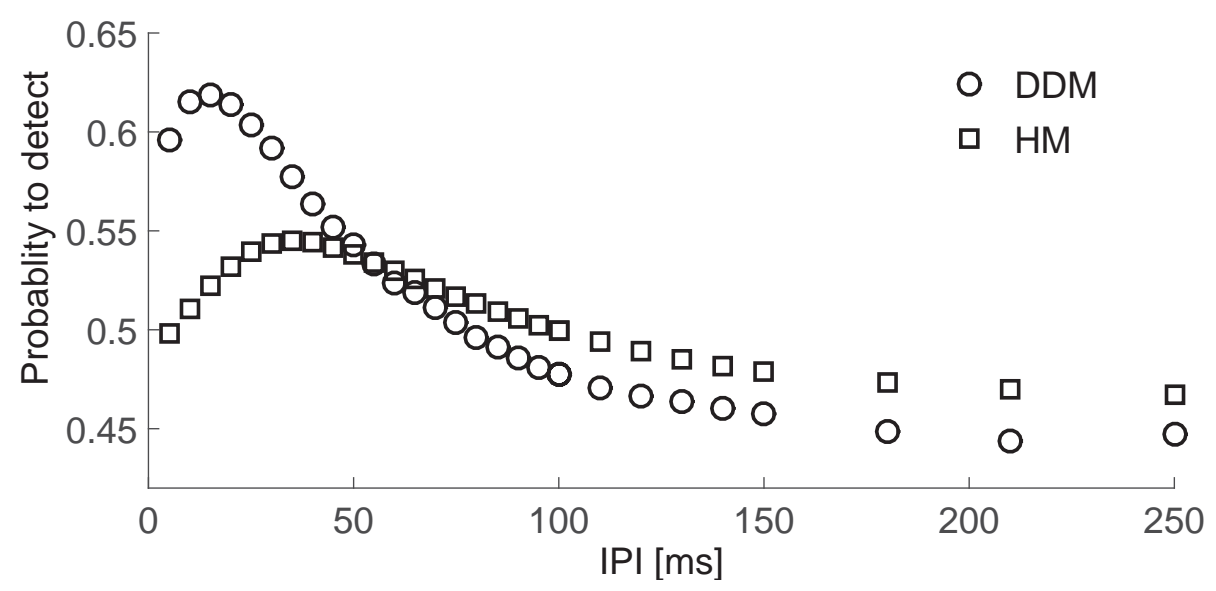

Figure 2.10: Model-simulated non-monotone effects of the $I P I$ on the probability to detect when in the DDM (circles) and the HM (squares) for $N o P=2$. The amplitude is fixed as $A=0.35 \mathrm{~mA}$, values of system parameters are identical to those used in Fig. 2.9. 


\subsection{Discussion}

We modeled a detection task with an electrocutaneous pulse-train stimulus. We derived a stochastic drift-diffusion model and a probabilistic hazard model with six lumped parameters characterising the underlying neurophysiological mechanisms. Using the models we explained the effects of temporal stimulus properties on thresholds in a human subject study. Both models have similar psychophysical curves, but the hazard model is computationally more convenient and hence more suitable for follow-up studies.

\subsubsection{Effects of temporal properties on detection thresholds}

The pulse-train stimulus has three temporal stimulus properties. Increasing $P W$, more nerve endings are activated, see Fig. 2.4B, which increases the activity of secondary neurons. This lowers the detection threshold in accordance with many other studies on neural activation (Mogyoros et al., 1996; Irnich, 2010). Increasing NoP, we noted two effects depending on the value of $I P I$. For large IPI, each pulse may be detected independently, increasing the detection probability. This is known as probability summation (Zwislocki, 1960; Gescheider et al., 1999). For shorter IPI, temporal summation of neural responses may further decrease the detection threshold. Our model differs from an earlier one by Zwislocki (1960), as we describe also trial-to-trial variability, accounting for a wide range of IPI values. Our experimental detection thresholds showed only a small increase when changing IPI from 10 to $50 \mathrm{~ms}$, which was at the significance level. This differs from the phenomenon observed in another study of tactile sensory processing (Gescheider et al., 1999). One possible explanation is based on the non-monotonic relationship from our model simulations due to the following reasons. First, tactile sensory processing relies mostly on $\mathrm{A} \beta$-fibers. The $\mathrm{A} \delta$-fibers activated by the electrocutaneous stimulation, differ in two aspects: their intrinsic neurophysiologcal properties and the neurophysiologcal characteristics of central neurons located in different laminae in the dorsal horn (Todd, 2010). Such differences could be reflected by the time constant of $200 \mathrm{~ms}$ for neural response used in (Gescheider et al., 1999), which is much larger than the time constants of afferent fibers and secondary neurons in the nociceptive system (Mogyoros et al., 1996; Weng et al., 2006). Second, Gescheider et al. (1999) utilized a merely deterministic model for the neural response, which did 
not account for the noisy neural activity with paired pulses. Third, the agreement on the non-monotone IPI effect on the detection probability between our model simulation and (Blumenthal et al., 1987) further supports our hypothetical explanation of the small IPI effect on detection thresholds. Future experiments could use a wider range for $I P I$ to study the effect of $I P I$ in more detail.

Other processes, such as threshold noise (Coombes et al., 2011), could also account for trial-to-trial variability. It is unclear what the plausible autocorrelation of the noise should be. Including threshold noise would also increase the number of lumped parameters by introducing parameters to characterize the autocorrelation. In addition, we encounter the same difficulty as for the DDM when we want to determine the distribution of the first passage times (FPTs) to compute the psychophysical function. Because of a lack of an analytically tractable expression of the distribution of the FPTs (Ricciardi and Sato, 1986; Di Nardo et al., 2000), model-based detection thresholds can only be simulated by generating a large set of realizations of threshold noise. Hence, a model considering threshold noise would be computationally expensive. This restricts the usage of a model with threshold noise in follow-up studies, e.g. parameter estimation.

\subsubsection{Interpretation of lumped parameters}

In both models, six lumped parameters characterize peripheral and central mechanisms. In the hazard model, the time constant $\tau_{1}$ and the threshold $\alpha_{1}$ affect the activation of peripheral fibers. The time constant $\tau_{2}$ and the firing rate $\lambda_{L}$ describe central properties. The threshold $\alpha_{L}$ and the slope parameter $\sigma_{L}$ depend on peripheral and central subsystems. The physical quantities $h, C_{1}, C_{2}, \alpha_{h}$, and $\sigma_{h}$ occur solely in the lumped parameters $\alpha_{1}, \tau_{1}, \tau_{2}, \alpha_{L}$, and $\sigma_{L}$, respectively. When one of these lumped parameters changes, one can attribute this to the corresponding physical quantity. For other physical quantities, this may not be the case. We discuss two pathological phenomena: hyperalgesia and central sensitization. When considering several possible causes of hyperalgesia (Sandkühler, 2009) with either a change in excitability of afferent fibers or secondary neurons or a change in synaptic strength, we can determine the corresponding change in the lumped parameters. Membrane excitability of afferent fibers and secondary neurons, and synaptic strength are characterized by $\kappa, K$, and $\bar{g}$, respectively. In peripheral activation, increased peripheral excitability $\kappa$ reflects in the simultaneous decrease in $\alpha_{1} \propto \kappa^{-1}, \alpha_{L} \propto \kappa^{-1}$ and 
$\sigma_{L} \propto \kappa^{-1}$. In central processing, the product of $\bar{g}$ and $K$ can be considered as the compound excitability of synapses and membranes of secondary neurons. As the lumped parameters $\alpha_{L}, \sigma_{L} \propto(\bar{g} K)^{-1}$, lower values of $\alpha_{L}$ and $\sigma_{L}$ indicate a higher compound excitability. However, individual contributions of $\bar{g}$ or $K$ to the compound excitability cannot be distinguished from these lumped parameters. In addition, central sensitization can manifest itself as a reduced outward flux of potassium ions of secondary neurons (Latremoliere and Woolf, 2009). This inhibition can be cast in a decrease of $G_{2}$. Such a decreased value of $G_{2}$ would result in $\tau_{2} \propto G_{2}^{-1}$ increases and both $\alpha_{L} \propto G_{2}$ and $\sigma_{L} \propto G_{2}$ decrease simultaneously. So different patterns of changes of these lumped parameters reflect distinguishable changes in either peripheral activation or central processing. Hence, these lumped parameters may be used in a patient-specific interpretation of (mal-)functioning in the nociceptive system. In addition, such understanding of effects of parameters would be used to guide new experimental design based on the model, such as predicting the effect of certain medication on thresholds based on modulation of synaptic efficiency in the dorsal horn. When such experimental measurements with detection thresholds under a perturbed nociceptive system become available, in turn, our understanding of parts of nociceptive processing might advance as well. As the model behaviour depends nonlinearly on multiple parameters, effects of these system parameters on detection thresholds will be a subject of future work.

\subsubsection{Model identifiability}

For a practical application of our modeling study one needs to infer system parameters from psychophysical measurements, as most of the lumped parameters are not measurable in a direct way. Obviously, we will not be able to determine the physical quantities, but only the lumped parameters. Here, we have chosen parameter sets to match model-based thresholds to experimentally obtained values, since an appropriate parameter estimation procedure is currently lacking. Standard system identification techniques are based on time-varying input and output (Ljung, 1999). In contrast, QST for pain diagnosis and monitoring yields psychophysical characteristics like thresholds rather than time varying measurements (Wilder-Smith and Arendt-Nielsen, 2006; Doll et al., 2014). In future work, we will investigate whether one can use these characteristics or stimulus-response pairs for system identification. The hazard model provides a good starting point, as it is efficient and captures the experimental effects of temporal stimulus properties. However, different from 
the logistic curve which is a generalized linear model, the nonlinearity in the HM challenges the assessment of model identifiability. Prior to performing parameter estimation for possible diagnostic purposes, the identifiability of the hazard model should be explored, i.e. a unique estimate can be determined provided sufficient information is available (Bellu et al., 2007; Raue et al., 2009). In our setting, it is a challenge to design suitable combinations of temporal stimulus properties for parameter identifiability.

\subsubsection{Model extensions}

So far, we have considered the essential neural mechanisms of stimulus processing in the ascending pathway. With respect to fiber activation, our model could be extended in two ways. First, we simplified the discrete number of activated nerve endings by the continuous variable $N_{r}$ (Eq. (2.4)). However, as both the strength of the induced electric field and the density of nerve endings determine this number, it is a challenge to improve this approximation for human subjects. If the $\mathrm{A} \delta$-nerve endings would be sparsely distributed, we recommend to minimize variations of the electrode-skin interface in experiments. Second, we assumed robust spike propagation and small variability in conduction velocity, because of the myelination of normal $\mathrm{A} \delta$-fibers. However, a demyelinating disease could amplify the contribution of these two factors on the post-synaptic activity, making these two terms necessary. Hence, on the one hand, more work is required to adequately describe the mechanisms for patients with a demyelinating disease; on the other hand, using our model, we suggest to use the presence of demyelinating disease as an exclusion criterion. Short-term plasticity is another process relevant for sensory synaptic transmission. We have not included this mechanism in our current model for several reasons. First, experimental evidence is collected for various synapses between afferent fibers and different laminae in the dorsal horn, see the review (Luo et al., 2014). As both synaptic depression and facilitation may occur, the net effect is uncertain. Second, our model already explains the effect of the IPI on detection thresholds. Shortterm plasticity may interfere with temporal summation, but both the data and the model suggest a small effect of the IPI. Nevertheless, if new experimental data provide conclusive evidence on the net effect due to the short-term plasticity, such an effect can be effectively modeled by modifying (2.7) such that $\bar{g}$ depends on IPI. Third, chronification of pain states is accompanied by the long-term plasticity, e.g. central sensitization. Such clinical relevance draws more attention on the long-term 
plasticity rather than short-term forms. To induce and maintain central sensitization, NMDA receptors (NMDAR) play an important role (Woolf and Thompson, 1991). Our models do not represent signal transduction, like protein kinase in postsynaptic neurons induced by the influx of calcium ions via NMDAR (Latremoliere and Woolf, 2009). Such mechanisms affect membrane excitability of secondary neurons and synaptic strength. Their compound effect is characterized by the two lumped parameters $\alpha_{L}$ and $\sigma_{L}$. Hence, parameters in the hazard model can still reflect the (mal-)functioning caused by involvement of NMDAR. An extended model representing more mechanisms due to NMDAR might increase insights into central sensitization. However, the substantially increased number of parameters for complex signaling pathways would challenge parameter estimation using psychophysical data from QST.

In addition, stimulus processing can be modulated by the descending pathway, but under normal circumstances it is inactive. Yet, it is clinically important as its malfunctioning may be related to chronic pain (Yarnitsky, 2010). The descending pathway may be activated by a conditioning stimulus such as the Cold Pressor Test (CPT) through Conditioned Pain Modulation (CPM) (Pud et al., 2009; Yarnitsky et al., 2010). It has been shown that the CPT leads to temporally increased detection thresholds (Doll et al., 2014). It is possible to incorporate descending inhibition to stimulus processing along the ascending pathway although the precise form, multiplicative due to shunting or additive due to normal inhibition, is unknown. If parameters of the ascending system can be estimated, it would then encourage to identify the descending pathway. 
3 


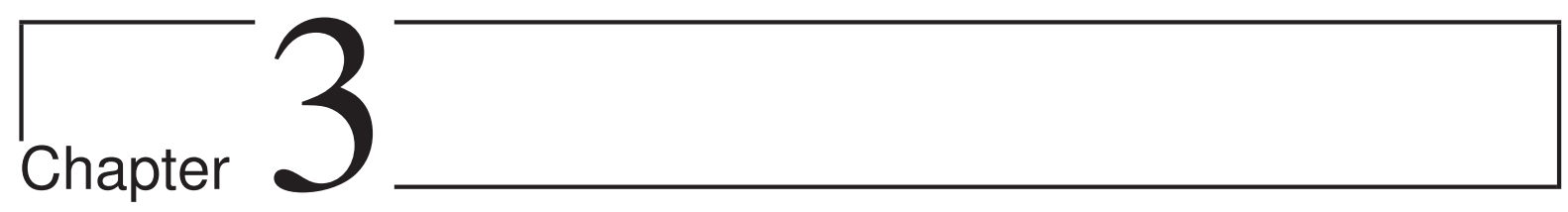

Dependence of nociceptive detection thresholds on physiological parameters and capsaicin-induced neuroplasticity: a computational study 


\section{Abstract}

Physiological properties of peripheral and central nociceptive subsystems can be altered over time due to medical interventions. The effective change for the whole nociceptive system can be reflected in changes of psychophysical characteristics, e.g. detection thresholds. However, it is challenging to separate contributions of distinct altered mechanisms with thresholds measurements. Here, we aim to understand how these alterations affect $\mathrm{A} \delta$ fiber-mediated nociceptive detection of electrocutaneous stimuli. First, with a neurophysiology-based model, we study effects of single model parameters on detection thresholds. Second, we derive a quantity of model parameters determining possible relations between detection thresholds and the interpulse interval for double-pulse stimuli. Third, in a case study with topical capsaicin treatment, we translate neuroplasticity into plausible changes of model parameters. Model-based simulations agree to experimental patterns of changes in detection thresholds. The simulations with individual forms of neuroplasticity confirm that nerve degeneration is the dominant mechanism for capsaicin-induced increases in detection thresholds. In addition, our study suggests that capsaicin-induced central plasticity may last at least one month. 


\subsection{Introduction}

The nociceptive system processes pain-related information and its function results from the delicate balance of a myriad of mechanisms (Coutaux et al., 2005; Sandkühler, 2009; Latremoliere and Woolf, 2009). Clinical interventions can perturb or recover this balance in multiple ways (Kyranou and Puntillo, 2012). Changes in the whole nociceptive system may be assessed with quantitative sensory testing (Wilder-Smith, 2002; Maier et al., 2010). However, separate contributions of distinct mechanisms are difficult to assess, which may be unraveled partially by using a modeling approach accounting for the relevant mechanisms.

The nociceptive system contains multiple pathways to detect noxious stimuli, i.e. actually or potentially tissue damaging events. The peripheral paths start with myelinated $\mathrm{A} \delta$ - and non-myelinated C-fibers, e.g. with nerve endings in the skin, responding to such stimuli. These fibers project to secondary neurons in the dorsal horn. Central terminals of both types of fibers contact secondary neurons extensively within the superficial dorsal horn, where nociceptive information is relayed to supra-spinal structures (Todd, 2010). Changes in the neurophysiological properties of both peripheral and central nociceptive subsystems can be induced by disease, clinical interventions or experimental conditioning stimuli, and can result in sensitization manifesting itself as hyperalgesia (Sandkühler, 2009). Sensitization is hypothesized to be one important factor leading to persistent pain (Latremoliere and Woolf, 2009). For an improved treatment of persistent pain, it is important to understand the individual and combined contributions of peripheral and central nociceptive processing. To study neuroplasticity underlying sensitization processes, we need to preferentially measure activation of nociceptive subsystems. Electrocutaneous stimulation at low stimulus amplitudes achieves this preferential activation of A $\delta$-fibers (Inui et al., 2002; Mouraux et al., 2010; Mørch et al., 2011; Steenbergen et al., 2012). Furthermore, electrical stimulation can be applied as a square wave pulse train characterized by three temporal properties, the number of pulses $\left(N_{o P}\right)$, the interpulse interval $(I P I)$ and the pulse width $(P W)$. The relation between the detection probability and stimulus amplitudes can be studied with fixed temporal properties. This relation, referred to as the psychometric function, is different for various values of temporal properties. A short $P W$ (i.e. $<2 \mathrm{~ms}$ ) controls the activation of $\mathrm{A} \delta$ fibers, while the $I P I$ ranging in tens of milliseconds contributes to temporal summation of neuronal activity of dorsal horn neurons. To study nocicep- 
tive processing underlying the detection task, we developed a computational model accounting for activation of $\mathrm{A} \delta$-fibers and central processing by secondary dorsal horn neurons (Yang et al., 2015a). This computational model is consistent with the principle of probability summation in psychophysical studies, which we refer to as the hazard model (HM). The computational convenience of the HM may facilitate further model-based studies. In addition, the model replicated the experimentally observed dependence of detection thresholds on temporal stimulus properties for healthy subjects. However, it was not addressed how changes of parameters due to neuroplasticity affect detection thresholds.

As a tool in pain research and as a therapeutic agent, capsaicin, the pungent substance in chili peppers, is widely used due to its capability to induce multiple forms of plasticity (O'Neill et al., 2012). Topical application of capsaicin is used as an experimental human pain model altering normal nociceptive functioning (Schmelz and Kress, 1996; Petersen and Rowbotham, 1999), accompanied by changes in peripheral and central nociceptive properties. Topical application of capsaicin activates afferent fibers via the transient receptor potential vanilloid 1 (TRPV1) by increasing calcium and sodium influx into peripheral fibers (O'Neill et al., 2012). A high level of intracellular calcium ions causes the degeneration of nerve endings. In addition to structural changes, activation of C-fibers triggers the release of neuropeptides leading to sensitization of peripheral nerve endings and dorsal horn neurons (Coutaux et al., 2005; Todd, 2010). A significant effect of 8\%-topical capsaicin treatment on detection thresholds was observed over three months in a human subject study for healthy subjects (Doll et al., 2015c). In addition, different patterns of changes of detection thresholds for single- and double-pulse stimuli were observed, shown in Fig. 3.1. However, a mechanistic explanation is not straightforward as changes in multiple subsystems may be involved.

Here, we propose and demonstrate a model-based approach to understand the effects of neuroplasticity on nociceptive detection of electrocutaneous stimuli. With the hazard model, we study how variations in single model parameters affect detection thresholds by parameter sweeping. Next, we find that the dependence of detection thresholds on the IPI for double-pulse stimuli can be non-monotone, for which we derive an explicit condition. As a case study using 8\%-topical capsaicin treatment, we translate neuroplasticity into plausible perturbations of model parameters over three months. We compare patterns of changes in model-based detection thresholds with experimentally obtained patterns qualitatively. We discuss how our 


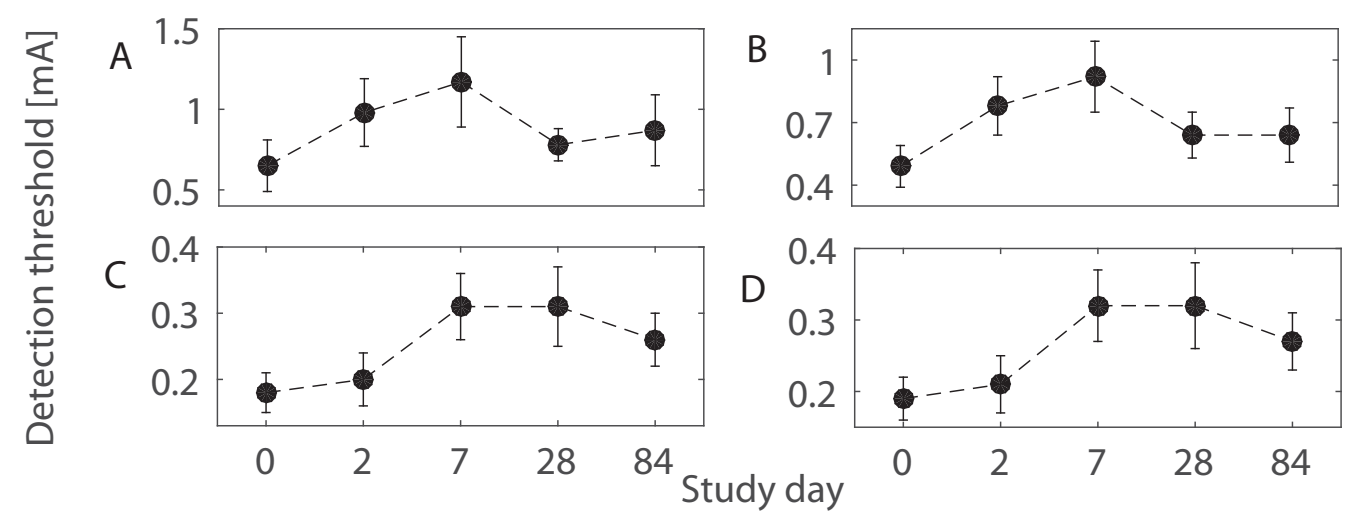

Figure 3.1: Detection thresholds (mean \pm SEM) using the four combinations of temporal properties on five study days before and after one-hour capsaicin treatment. A: $(N o P=1$, $P W=0.21 \mathrm{~ms}), \mathrm{B}:(N o P=1, P W=0.525 \mathrm{~ms}), \mathrm{C}:(N o P=2, I P I=20 \mathrm{~ms}, P W=$ $0.525 \mathrm{~ms})$, and D: $(N o P=2, I P I=50 \mathrm{~ms}, P W=0.525 \mathrm{~ms})$, respectively.

findings could be used for future experiments or further exploration of capsaicininduced effects relevant to pain management.

\subsection{Material and methods}

We begin with a brief description of the computational model of A $\delta$-fibermediated nociception detection to electrocutaneous stimuli. Next, to understand possible neuroplasticity, we vary each model parameter to study its effects on detection thresholds. For double-pulse stimuli, we derive a quantity determining the monotonicity of detection thresholds with respect to the interpulse interval.

\subsubsection{Computational modeling of nociceptive detection of elec- trocutaneous stimulation}

In the experimental setup, electrocutaneous stimuli are delivered by an intraepidermal needle electrode (Steenbergen et al., 2012; Doll et al., 2014). The experimental details were reported in (Doll et al., 2015c). For each stimulus, the binary response $R$ was recorded, i.e. $R=1$ if the stimulus was detected, $R=0$ if not. Fixing the three temporal properties (NoP, IPI and $P W)$, a psychometric function describes the conditional probability to detect a stimulus with the amplitude $(A)$, i.e. $\operatorname{Pr}(R=1 \mid A)$. In our modeling study, we denote $\Psi(A)$ as the model-based 
psychometric function. The binary response in a single trial can be simulated by drawing a random number $\xi$ from a standard uniform distribution. The response is $R=1$ when $\xi<\Psi(A)$, indicating that the stimulus is detected, and $R=0$ otherwise. We denote the model-based detection threshold by $A_{50}$ and it is implicitly defined by $\Psi\left(A_{50}\right)=0.5$. It is well defined if $\Psi(0)<0.5$ and then $A_{50}$ can be determined numerically.

Here, we briefly describe the model, for more details see (Yang et al., 2015a). Peripheral activation by the electrical stimulus is described by the threshold-linear function $\left[f_{A}-\alpha_{1}\right]_{+}:=\pi\left(f_{A}-\alpha_{1}\right) H\left(f_{A}-\alpha_{1}\right)$, where $f_{A}:=A\left(1-\exp \left(-\frac{P W}{\tau_{1}}\right)\right)$ and $H(\cdot)$ is a Heaviside step function; $\tau_{1}$ and $\alpha_{1}$ are the time constant and the activation threshold of afferent fibers, respectively. Next, through synaptic connections, an excitatory post-synaptic current $I_{p}^{*}(t)$ is induced

$$
I_{p}^{*}(t)=\frac{\left[f_{A}-\alpha_{1}\right]_{+}}{\tau_{s}} \sum_{k=0}^{N o P-1} \exp \left(-\frac{t-k I P I}{\tau_{s}}\right) H(t),
$$

with time constant $\tau_{s}=1.5 \mathrm{~ms}$ (Gabbiani et al., 1994). This drives the postsynaptic potential $x(t)$ of a secondary dorsal horn neuron, which we model as a leaky integrator

$$
\tau_{2} \dot{x}=-x+I_{p}^{*}(t), \quad x(0)=0 .
$$

The value of the time constant $\tau_{2}$ is roughly several tens of milliseconds (Prescott and Koninck, 2002; Weng et al., 2006). This noise-free post-synaptic potential is converted into an instantaneous firing rate through a non-homogeneous Poisson process (Plesser and Gerstner, 2000)

$$
\lambda(t)=\lambda_{L}\left(1+\exp \left(\frac{\alpha_{L}-x(t)}{\sigma_{L}}\right)\right)^{-1} .
$$

Here the lumped parameters $\alpha_{L}, \sigma_{L}$ and $\lambda_{L}$ represent the threshold, the slope parameter and the maximal firing rate, respectively. The expected value of the number of spikes during a trial interval of duration $T$ is

$$
\lambda_{T}=\int_{0}^{T} \lambda(t) d t
$$

Our developed model assumed that the binary response $R$ equaled one if at least one secondary neuron generated an action potential during the trial interval $T$ (Yang et al., 2015a). So the model-based psychometric function is given by

$$
\Psi=1-\exp \left(-\lambda_{T}\right)
$$


Our model contains six lumped parameters, which depend on several physical quantities, characterizing peripheral and central nociceptive subsystems. We summarize descriptions of physical quantities as well as expressions of lumped parameters with respect to physical quantities in Table 3.1, according to (Yang et al., 2015a).

Table 3.1: Summary of physical and lumped parameters in the computational model

\begin{tabular}{|c|c|c|c|}
\hline \multicolumn{4}{|c|}{ PHYSICAL QUANTITIES } \\
\hline Symbol & \multicolumn{3}{|c|}{ Description } \\
\hline$V_{t h}$ & \multicolumn{3}{|l|}{ firing threshold of afferent fibers } \\
\hline$c_{0}$ & \multicolumn{3}{|c|}{ conductivity of the superficial tissue under skin } \\
\hline$c_{1}$ & \multicolumn{3}{|c|}{ electrical resistance of nerve endings per unit length } \\
\hline$C_{1}$ & \multicolumn{3}{|c|}{ membrane capacitance of nerve endings } \\
\hline$G_{1}$ & \multicolumn{3}{|l|}{ membrane conductance of nerve endings } \\
\hline$\rho$ & \multicolumn{3}{|l|}{ surface density of nerve endings } \\
\hline$h$ & \multicolumn{3}{|c|}{ distance between nerve endings and the electrode } \\
\hline $\bar{g}$ & \multicolumn{3}{|c|}{ maximal conductance of the AMPA-mediated synapse } \\
\hline$K$ & \multicolumn{3}{|c|}{ potential gradient between the post-synaptic potential and the AMPA-reversal potential } \\
\hline$C_{2}$ & \multicolumn{3}{|c|}{ membrane capacitance of secondary neurons } \\
\hline$G_{2}$ & \multicolumn{3}{|l|}{ membrane conductance of secondary neurons } \\
\hline$\alpha_{h}$ & \multicolumn{3}{|c|}{ activation threshold of secondary neurons } \\
\hline$\sigma_{h}$ & \multicolumn{3}{|c|}{ slope parameter of the activation of secondary neurons } \\
\hline$l$ & \multicolumn{3}{|c|}{ number of secondary neurons } \\
\hline$\lambda_{h}$ & \multicolumn{3}{|c|}{ maximal firing rate of single secondary neurons } \\
\hline \multicolumn{4}{|c|}{$\begin{array}{l}\text { LUMPED PARAMETERS } \\
\end{array}$} \\
\hline Symbol & Description & Expression & Reference value \\
\hline$\alpha_{1}$ & activation threshold of afferent fibers & $\left(4 \pi c_{0} c_{1} G_{1} V_{t h}\right) h^{2}$ & 0.125 \\
\hline$\tau_{1}$ & time constant of afferent fibers & $C_{1} G_{1}^{-1}$ & 0.2 \\
\hline$\tau_{2}$ & time constant of secondary neurons & $C_{2} G_{2}^{-1}$ & 45 \\
\hline$\alpha_{L}$ & activation threshold of secondary neurons & $\left(\frac{8}{3} \pi c_{0} c_{1} G_{1} G_{2} V_{t h}\right) \alpha_{h}(\rho \bar{g} K)^{-1}$ & 0.00417 \\
\hline$\sigma_{L}$ & slope parameter of firing rate function & $\left(\frac{8}{3} \pi c_{0} c_{1} G_{1} G_{2} V_{t h}\right) \sigma_{h}(\rho \bar{g} K)^{-1}$ & $8.33 \times 10^{-5}$ \\
\hline$\lambda_{L}$ & the maximal population firing rate & $l \lambda_{h}$ & 0.01 \\
\hline
\end{tabular}

\subsubsection{Effects of single model parameters on detection thresh- olds}

Here, we study how the model-based detection threshold changes by sweeping single physical or lumped parameters around reference values. Table 3.1 gives the dependence of lumped parameters on physical quantities. There exist two kinds of 
redundancy. One is among the physical quantities. There are three subgroups of such parameters: $\left\{V_{t h}, c_{0}, c_{1}\right\},\{\rho, \bar{g}, K\}$, and $\left\{l, \lambda_{h}\right\}$. The other kind of redundancy reflects the relation between physical quantities and six lumped parameters: $h$ and $\alpha_{1} ; C_{1}$ and $\tau_{1} ; C_{2}$ and $\tau_{2} ; \alpha_{h}$ and $\alpha_{L} ; \sigma_{h}$ and $\sigma_{L} ;$ and $\left\{l, \lambda_{h}\right\}$ and $\lambda_{L}$. Due to the redundancy, we only give reference values of lumped parameters in Table 3.1 rather than physical quantities. We set $\alpha_{1}=0.125 \mathrm{~mA}, \tau_{1}=0.2 \mathrm{~ms}, \tau_{2}=45 \mathrm{~ms}$, $\alpha_{L}=0.00417 \mathrm{~A} / \mathrm{s}, \sigma_{L}=8.33 \times 10^{-5} \mathrm{~A} / \mathrm{s}$, and $\lambda_{L}=0.01 \mathrm{kHz}$. The magnitudes of the time constants $\tau_{1}$ and $\tau_{2}$ are similar to those from (Mogyoros et al., 1996; Prescott and Koninck, 2002; Weng et al., 2006). The values of the remaining four lumped parameters are chosen to produce model-based detection thresholds similar to experimentally observed ones. Based on the expressions in Table 3.1, it is sufficient to consider ten physical quantities: $\left(V_{t h}, C_{1}, G_{1}, \rho, h, C_{2}, G_{2}, \alpha_{h}, \sigma_{h}, \lambda_{h}\right)$ to cover all parameters. We vary the effect of parameters with relative variations from $60 \%$ to $170 \%$ to the reference values. In model simulations, we use four combinations of temporal stimulus properties used in the experiments (Doll et al., 2015c): $(N o P=$ $1, P W=0.21 \mathrm{~ms}),(N o P=1, P W=0.525 \mathrm{~ms}),(N o P=2, I P I=20 \mathrm{~ms}$, $P W=0.525 \mathrm{~ms})$, and $(N o P=2, I P I=50 \mathrm{~ms}, P W=0.525 \mathrm{~ms})$. To illustrate a possible non-monotone dependence of detection thresholds on $\sigma_{h}$ or $\sigma_{L}$., we use another combination of temporal stimulus properties: $(N o P=2, I P I=30 \mathrm{~ms}$, $P W=0.525 \mathrm{~ms})$.

\subsubsection{Effects of temporal stimulus properties on detection thresholds}

Here, we analyze the effects of temporal stimulus properties on the detection threshold, in particular the IPI. As we noted before in (Yang et al., 2015a), the threshold decreases for increasing $P W$, because of enhanced fiber activation giving more impulses to secondary neurons. For multiple pulses, the detection threshold is also lower compared to single-pulse stimuli. For double-pulse stimuli, our previous modeling study suggests that the dependence $A_{50}(I P I)$ could be non-monotone. In the following, we derive a quantity of model parameters to determine this monotonicity.

For simplicity we will restrict ourselves to the case $N o P=2$. The effect for more pulses is similar, but less pronounced, and the analysis more involved. First, we assume $\tau_{s} \rightarrow 0$ as $\tau_{s} \ll \tau_{2}$ based on (Gabbiani et al., 1994; Prescott and Koninck, 
2002). So the lumped postsynaptic potential in (3.2) can be simplified to

$$
x^{0}(t)=B \sum_{k=0}^{N o P-1} \exp \left(-\frac{t-k I P I}{\tau_{2}}\right) H(t-k I P I),
$$

where $B=\frac{\pi\left[f_{A}-\alpha_{1}\right]_{+}}{\tau_{2}}$.

Second, we consider the limit $\sigma_{h} \rightarrow 0$, resulting in $\sigma_{L} \rightarrow 0$. This simplifies the analysis, as there is only a contribution to the integral $\lambda_{T}$ if $x(t)>\alpha_{L}, t \in(0, T)$. Indeed, as (3.3) becomes a step function, only the total time $\Delta T$ above the threshold $\alpha_{L}$ has to be determined. Now, we have three distinct cases to reach the threshold $\alpha_{L}$. First, the activity would be above threshold after the second pulse, but not the first. Second, both pulses may lead to supra-threshold activity but during two separate time intervals. Third, if a single pulse leads to supra-threshold activity, we may have one interval if $x\left(I P I^{-}\right)>\alpha_{L}$. The time $\Delta T_{i}, i=1,2,3$ above $\alpha_{L}$ can be calculated explicitly as

$$
\begin{array}{r}
\Delta T_{1}=\tau_{2}\left(\log \left(1+\exp \left(-I P I / \tau_{2}\right)-\log \left(\frac{\alpha_{L}}{B}\right)\right),\right. \\
\Delta T_{2}=\tau_{2}\left(\log \left(1+\exp \left(-I P I / \tau_{2}\right)-2 \log \left(\frac{\alpha_{L}}{B}\right)\right),\right. \\
\Delta T_{3}=I P I+\tau_{2}\left(\log \left(1+\exp \left(-I P I / \tau_{2}\right)-\log \left(\frac{\alpha_{L}}{B}\right)\right) .\right.
\end{array}
$$

To demonstrate the three cases, we use double-pulse stimuli with $I P I=20 \mathrm{~ms}$ and $P W=0.525 \mathrm{~ms}$. We simulate lumped postsynaptic potential of the HM at amplitudes $A=0.19,0.22$ and $0.26 \mathrm{~mA}$, where we set lumped parameters at the reference values as in Table 3.1 .

We consider model-based detection thresholds in these three cases. From (3.4), the detection threshold $A_{50}$ and the IPI satisfy the implicit equation $\lambda_{L} \Delta T=$ $\log (2)$. If the $I P I$ is large, then the time above threshold is always given by $\Delta T_{2}$. Decreasing $I P I$, the detection threshold $A_{50}$ may change such that either $\Delta T_{1}$ or $\Delta T_{3}$ should be used. The value of IPI where we switch from the second case to the first, is given by

$$
I P I_{21}=-\tau_{2} \log \left(2^{1 /\left(\tau_{2} \lambda_{L}\right)}-1\right)
$$

and to the third case by

$$
I P I_{23}=\tau_{2} \log \left(\sqrt{2^{1 /\left(\tau_{2} \lambda_{L}\right)}+\frac{1}{4}}-\frac{1}{2}\right) .
$$

Now either $\lambda_{L} \tau_{2}>1$, and then $I P I_{21}$ is negative and $I P I_{23}$ is positive, or $\lambda_{L} \tau_{2}<1$ and then $I P I_{23}$ is negative and $I P I_{21}$ is positive. So, for decreasing the $I P I$, we 
find either case 1 or case 3 , and this depends on whether $\lambda_{L} \tau_{2}$ is smaller or larger than one.

It is easy to see that $\Delta T_{1}, \Delta T_{2}$ are decreasing functions of $I P I$, and $\Delta T_{3}$ is an increasing function. It follows from the definition of the psychometric function (3.4) that $\partial A_{50}(I P I) / \partial I P I<0$ if $I P I<I P I_{23}$ and $\lambda_{L} \tau_{2}<1$ and the derivative is positive otherwise. Hence if $\lambda_{L} \tau_{2}<1$ the dependence of the detection threshold on the interpulse interval is non-monotone.

\subsubsection{Neuroplasticity induced by topical capsaicin treatment and relevant modeling}

We consider the observed patterns of changes in detection thresholds on five study days with one-hour capsaicin treatment over three months with $8 \%$ capsaicin treatment (Doll et al., 2015c), as illustrated in Fig. 3.1. First, we briefly review neuroplasticity caused by topical application of capsaicin. Second, with plausible changes in related model parameters, we study the patterns of changes in modelbased detection thresholds.

\subsubsection{Capsaicin-induced neuroplasticity}

Capsaicin activates the TRPV1-expressing $\mathrm{C}$ fibers and some A $\delta$ fibers (O'Neill et al., 2012). The application of a high-dose capsaicin patch results in high levels of intracellular calcium in nerve endings leading to mitochondrial dysfunction and retraction of nerve endings (Anand and Bley, 2011). In the periphery, neuropeptides, like substance $\mathrm{P}$ (SP) and calcitonin gene-related peptide (CGRP) are released from the activated peptidergic $\mathrm{C}$ fibers, resulting in neurogenic inflammation (Coutaux et al., 2005; Voscopoulos and Lema, 2010). This inflammation is accompanied by the release of inflammatory agents such as prostaglandin E2 and histamine. In turn, these agents may trigger intracellular signaling in nociceptors, through protein kinase A and C (PKA and PKC) (Khasar et al., 1999; Malmberg, 2000). These protein kinases can further modulate the properties of tetrodotoxin-resistant (TTXr) persistent sodium channels in nerve endings, shifting the activation curve of these channels to more hyperpolarized values (Gilchrist and Bosmans, 2012). So, the excitability of $\mathrm{A} \delta$-fibers may increase due to the presence of inflammatory agents. After a 60-minute application of an $8 \%$ capsaicin patch, it is common to observe 
application-site erythema. The erythema was reported to resolve within 1-3 days after the capsaicin patch treatment (FDA Center for Drug Evaluation and Research, 2009). This suggests that capsaicin-induced neurogenic inflammation could last up to three days. In the superficial dorsal horn, the central terminals of peptidergic C fibers modulate the secondary neurons via release of SP (Todd, 2010). SP can further activate intracellular signaling, e.g. PKA, PKC and extracellular-signalregulated kinases (ERK) in superficial dorsal horn neurons resulting in shorter and longer lasting neuroplasticity. The increased density of AMPA receptors enhances synaptic efficacy and the reduction of A-type potassium currents increases membrane excitability (Latremoliere and Woolf, 2009). These effects can last several hours in human experimental pain models (Sandkühler, 2009; Latremoliere and Woolf, 2009) and are considered to be transcription-independent processes. The longer lasting central neuroplasticity was suggested to be transcription-dependent possibly explaining the persistent pain state in pathological settings (Latremoliere and Woolf, 2009).

\subsubsection{Simulated detection thresholds over three months}

We model the capsaicin-induced effects by different perturbations of physical quantities for each study day. First, for degeneration of nerve endings and their regrowth, the intra-epidermal nerve fiber density $\rho$ is expected to decrease initially and then to return to baseline as also observed by Kennedy et al. (2010). In addition, as the nerve endings retract from the epidermis, their depth $h$ increases (O'Neill et al., 2012). Second, we increase the membrane excitability of nerve endings by lowering the firing threshold $V_{t h}$ on Day 2. This models the capsaicin-induced neurogenic inflammation, which in normal circumstances is resolved within a couple of days. Third, increases in membrane excitability and synaptic efficacy of the superficial dorsal horn neurons can be captured by decreasing $G_{2}$ and increasing $\bar{g}$, respectively. Summarizing, it is plausible that changes in the physical quantities $\rho, h, V_{t h}$, $G_{2}$ and $\bar{g}$ can account for the experimental pattern in detection thresholds.

We consider the values of the model parameters on Day 0 as baseline. We use the same reference values of lumped parameters in parameter sweeping as: $\alpha_{1}=$ $0.125 \mathrm{~mA}, \tau_{1}=0.2 \mathrm{~ms}, \tau_{2}=45 \mathrm{~ms}, \alpha_{L}=0.00417 \mathrm{~A} / \mathrm{s}, \sigma_{L}=8.33 \times 10^{-5} \mathrm{~A} / \mathrm{s}$, and $\lambda_{L}=0.01 \mathrm{kHz}$. The perturbation patterns are presented as multiplication factors $\left(r_{\rho}, r_{h}, r_{V_{t h}}, r_{G_{2}}\right.$, and $\left.r_{\bar{g}}\right)$ to the baseline values, see Table 3.2. So the actual lumped parameter values are changed according to the expressions in Table 3.1. A 
Table 3.2: Perturbation patterns of the ratios of five physical quantities.

\begin{tabular}{cccccc}
\hline Parameters & Day 0 & Day 2 & Day 7 & Day 28 & Day 84 \\
\hline$r_{\rho}$ & 1 & 0.172 & 0.21 & 0.371 & 0.80 \\
$r_{h}$ & 1 & 1.3 & 1.25 & 1.23 & 1.15 \\
$r_{V_{t h}}$ & 1 & 0.45 & 1 & 1 & 1 \\
$r_{G_{2}}$ & 1 & 0.92 & 0.9 & 0.96 & 0.98 \\
$r_{\bar{g}}$ & 1 & 1.832 & 4.608 & 3.234 & 1.4 \\
\hline
\end{tabular}

previous human study with the same administration of the capsaicin patch to thighs reported decreased fiber densities on Day 7 and 84 , to $21 \%$ and $80 \%$ of the baseline, respectively (Kennedy et al., 2010). Here, we set these two ratios for our model simulations. In addition, we need the densities on Day 2 and 28. Given that the regeneration of fiber densities approximately obeyed a linear relation with respect to time (Polydefkis et al., 2004), we determine the ratios of fiber densities by linear extrapolation and interpolation based on above densities on Day 7 and 84. Other changes in ratios are set to capture the experimentally obtained changes in detection thresholds in a systematic way, which also qualitatively agrees to fiber retraction (O'Neill et al., 2012), changes of peripheral excitability (Coutaux et al., 2005) and plasticity of the central subsystem (Latremoliere and Woolf, 2009). We compute the detection thresholds for each combination of temporal properties on each study day. To understand how changes in all five physical quantities act together, we also compute the thresholds with either peripheral or central functional plasticity omitted, or both.

\subsection{Results}

To explore effects of physiological parameters on detection thresholds, we present simulation results from parameter sweeping. Next, we emphasize the effect of the IPI on the detection threshold based on both our mathematical analysis and model simulations. Last, we show simulated detection thresholds on the five study days in the case study of topical capsaicin treatment. These simulations show qualitative agreement to change patterns of experimental thresholds. 


\subsubsection{Effects of single model parameters on detection thresh- olds}

Each panel in Fig. 3.2 illustrates the effect of a single model parameter on the detection threshold. Big markers show detection thresholds for the reference values in Table 3.1. The steeper the curve around the reference value is, the stronger the threshold depends on this parameter locally. These results show that detection thresholds can depend non-monotonically on physical quantities $C_{2}$ and $\sigma_{h}$ or lumped parameters $\tau_{2}$ or $\sigma_{L}$. For secondary neurons, morphological properties, i.e. the thickness and the surface of the membrane, determine $C_{2}$. It is not likely that $C_{2}$ changes much over time, and if it would, e.g. due to the cell growth, then also the membrane conductance $G_{2}$ is expected to change. As $\tau_{2}=C_{2} G_{2}^{-1}$, increases in both $C_{2}$ and $G_{2}^{-1}$ result in an invariant $\tau_{2}$. However, a change of the thickness of the membrane can lead to a change solely in the physical quantity $C_{2}$ or the lumped parameter $\tau_{2}$. For double-pulse stimuli, We notice that two combinations have very similar thresholds in Fig. 3.2. We comment on this in the next subsection. For $I P I=30 \mathrm{~ms}$ and $P W=0.525 \mathrm{~ms}$, we find non-monotone dependence of simulated detection thresholds on $\sigma_{h}$ or $\sigma_{L}$, see Fig. 3.3I. We also show dependency of the detection thresholds on other parameters in Fig. 3.3.

\subsubsection{Effect of the IPI on detection thresholds}

Using double-pulse stimuli at different amplitudes, we illustrate the three ways the lumped postsynaptic potential can cross the activation threshold $\alpha_{L}$ of central neurons, see Fig. 3.4. Next, as we analyzed the transition between the above different scenarios in section 3.2.3, the quantity $\lambda_{L} \tau_{2}$ determines the monotonicity of the detection threshold with respect to the IPI. Although this statement is obtained when $\sigma_{L} \rightarrow 0$ and $\tau_{s} \ll \tau_{2}$, we check the statement by simulating the hazard model also with non-zero $\sigma_{L}$ (i.e. $8.33 \times 10^{-5} \mathrm{~A} / \mathrm{s}$ ) and $\tau_{s}=1.5 \mathrm{~ms}$, see Fig. 3.5. Black and blue curves depict the relationship between the detection threshold and the $I P I$ with $\lambda_{L}=0.01$ and $0.05 \mathrm{kHz}$, respectively. To generate these plots, other five lumped parameters are set at reference values as in Table 3.1. We find that the simulated detection thresholds (dashed curves) follow the analytical expressions (plotted as solid curves) very closely. This demonstrates the validity of our analysis. As $\tau_{2}=C_{2} G_{2}^{-1}$ and $\lambda_{L}=l \lambda_{h}$, we note that changes in these four physical quantities could affect the monotonicity of detection thresholds. 


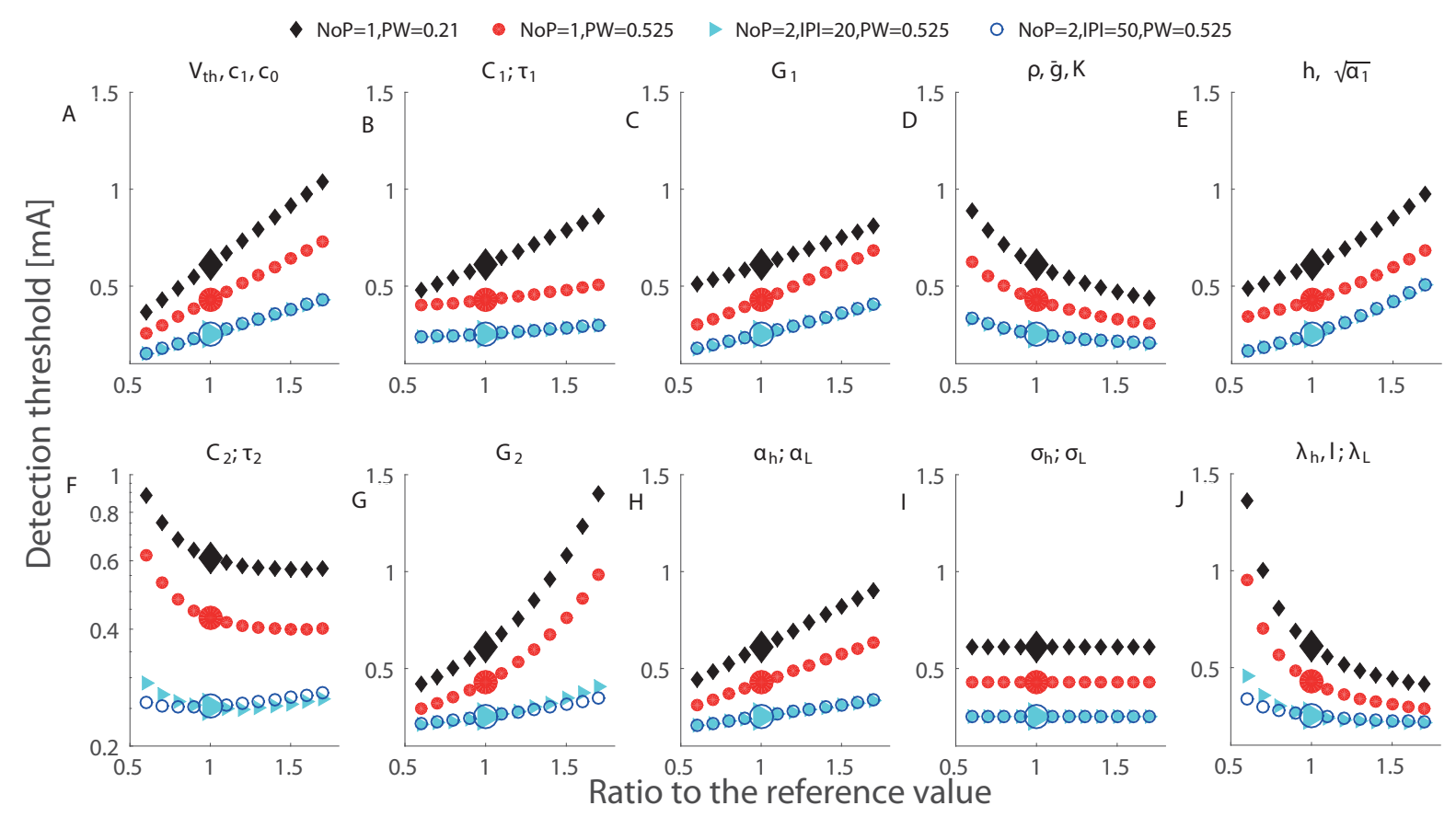

Figure 3.2: Simulated detection thresholds with perturbation of single parameters. Black: $N o P=1, P W=0.21 \mathrm{~ms}$; Red: $N o P=1, P W=0.525 \mathrm{~ms}$; Cyan: $N o P=2, I P I=20 \mathrm{~ms}$, $P W=0.525 \mathrm{~ms}$; and Blue: $N o P=2, I P I=50 \mathrm{~ms}, P W=0.525 \mathrm{~ms}$. The titles in each panel indicate which parameters are perturbed. In panel $\mathrm{F}$, the $\mathrm{y}$-axis has a log-scale for better visualization of the non-monotonicity of detection thresholds with respect to $C_{2}$ or $\tau_{2}$ using four temporal combinations.

\subsubsection{Simulated detection thresholds after topical capsaicin treatment}

We have computed the model-based detection thresholds for the perturbation pattern in Table 3.2. For each combination of temporal properties we show the threshold in Fig. 3.6 (black crosses). We see that the pattern is qualitatively similar as the experimental pattern in Fig. 3.1. For single-pulse stimuli, detection thresholds increase on Day 2 and 7, and for double-pulse stimuli, thresholds increase on Day 7 and 28. Also, for double-pulse stimuli on Day 84, the simulated detection thresholds are still larger than their baselines, which also agrees to the elevated thresholds compared to those on Day 0 (although not significant) shown in Fig. 3.1C and D.

To study different combinations of neuroplasticity with the model, we omit either peripheral or central functional plasticity, or both. We present corresponding simulated detection thresholds for the three cases, see the gray, red and blue markers in Fig. 3.6, respectively. For these three cases, we find much higher thresholds. 


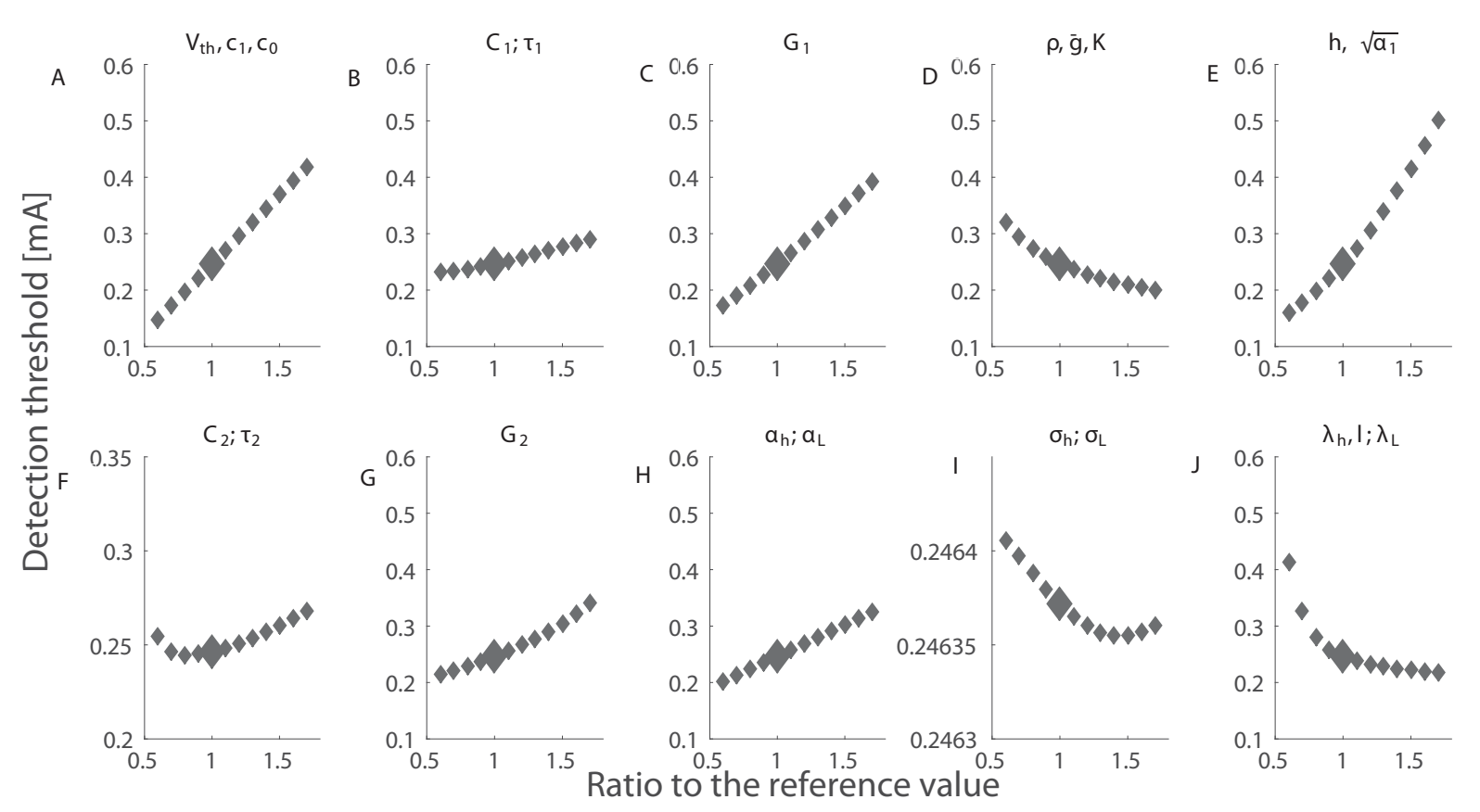

Figure 3.3: Simulated detection thresholds with perturbation of single parameters when $N o P=2, I P I=30 \mathrm{~ms}, P W=0.525 \mathrm{~ms}$. The titles in each panel indicate which parameters are perturbed. In panel $\mathrm{F}$ and $\mathrm{I}$, the $\mathrm{y}$-axis has a different scale for better visualization of the non-monotone effects of $C_{2}, \tau_{2}$ or $\sigma_{h}, \sigma_{L}$ on detection thresholds, respectively.

With only nerve degeneration (blue circles), the thresholds would increase on Day 2, 7 and 28 similarly for both single- and double-pulse stimuli. Adding only peripheral sensitization (red diamonds) results in different patterns, but these simulations do not agree to detection thresholds for single-pulse stimuli observed on Day 28. With central functional plasticity and nerve degeneration, simulation (gray squares) shows a quantitative disagreement for $N o P=2$ on Day 2 .

\subsection{Discussion}

We have investigated the effects of model parameters on detection thresholds with our computational model in terms of neuroplasticity. First, we demonstrated that the detection threshold varies monotonically for most single parameters except for $C_{2}, \sigma_{h}, \tau_{2}, \sigma_{L}$ involved in central nociceptive function. Second, we developed an analytical argument why detection thresholds may depend non-monotonically on the $I P I$. Third, we translated biologically plausible capsaicin-induced neuroplasticity into perturbations in values of model parameters. Agreement between simulations and observations of detection thresholds suggests the prospective usage of this com- 


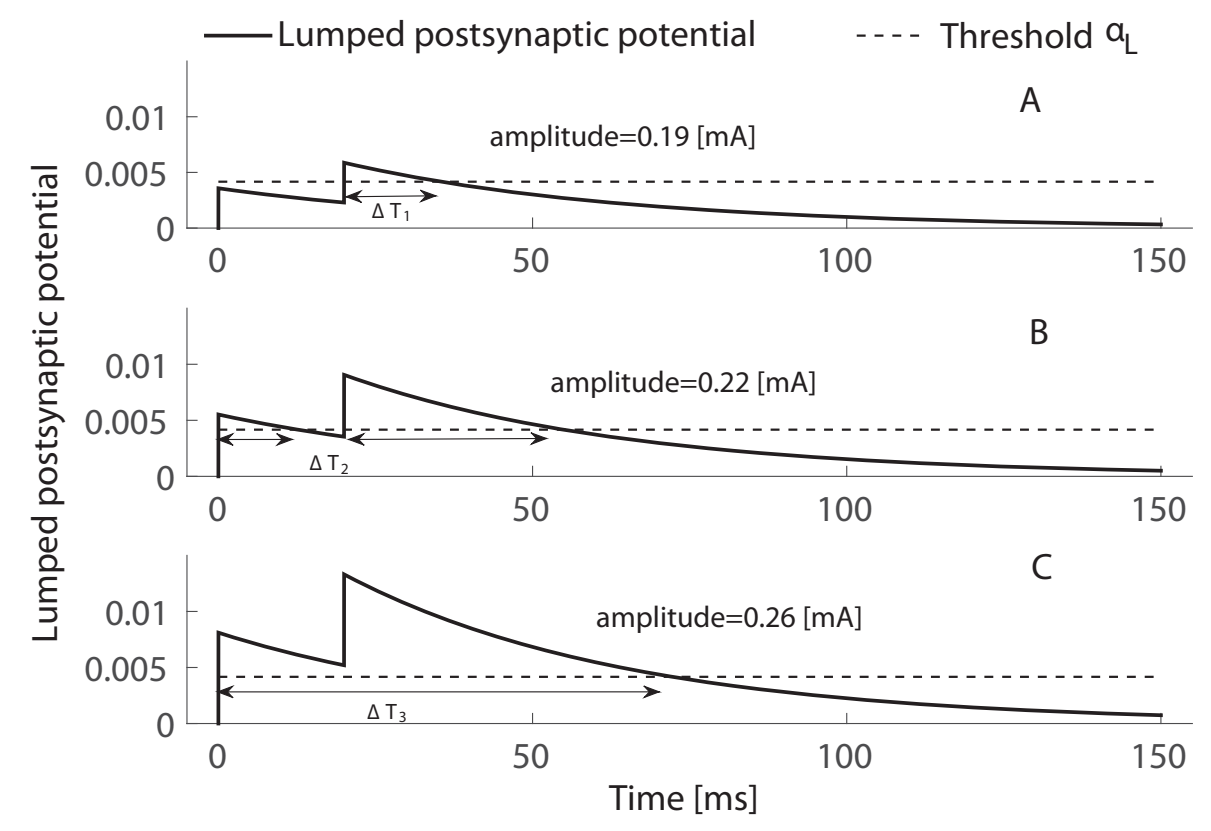

Figure 3.4: Three ways how the post-synaptic potential $x^{0}(t)$ (solid) can cross the threshold (dashed) when two pulses are applied. The above-threshold time $\Delta T$ may consist of one interval by crossing upon the second pulse only (A) or upon the first pulse staying above threshold until the second pulses (C). If the pulses are well separated in time $\Delta T$ consists of two intervals (B). Temporal properties are $N o P=2, I P I=20 \mathrm{~ms}$ and $P W=0.525 \mathrm{~ms}$, and the amplitude are varied, i.e. not equal to $A_{50}$.

putational model for a malfunctioning nociceptive system.

\subsubsection{Effects of parameters on detection thresholds}

Detection thresholds depend on most of single parameters in a monotone manner, see Fig. 3.2. On the other hand, the effects of different parameters on detection thresholds could be similar. For example, this occurs for the pair of physical quantities $C_{1}$ and $\alpha_{L}$ (equivalently, also lumped parameters $\tau_{1}$ and $\left.\alpha_{L}\right)$ when $(N o P=1$, $P W=0.21 \mathrm{~ms}),(N o P=2, I P I=20 \mathrm{~ms}, P W=0.525 \mathrm{~ms})$, or $(N o P=2$, $I P I=50 \mathrm{~ms}, P W=0.525 \mathrm{~ms}$ ), see Fig. 3.2B and $\mathrm{H}$. This similarity challenges identifiability of such model parameters, as they can compensate each other, resulting in equal detection thresholds. Hence, in future work like parameter estimation, we recommend to design more appropriate combinations of temporal properties to enrich the information of the data.

In a previous work (Doll et al., 2015a), varying the interpulse interval from 10 


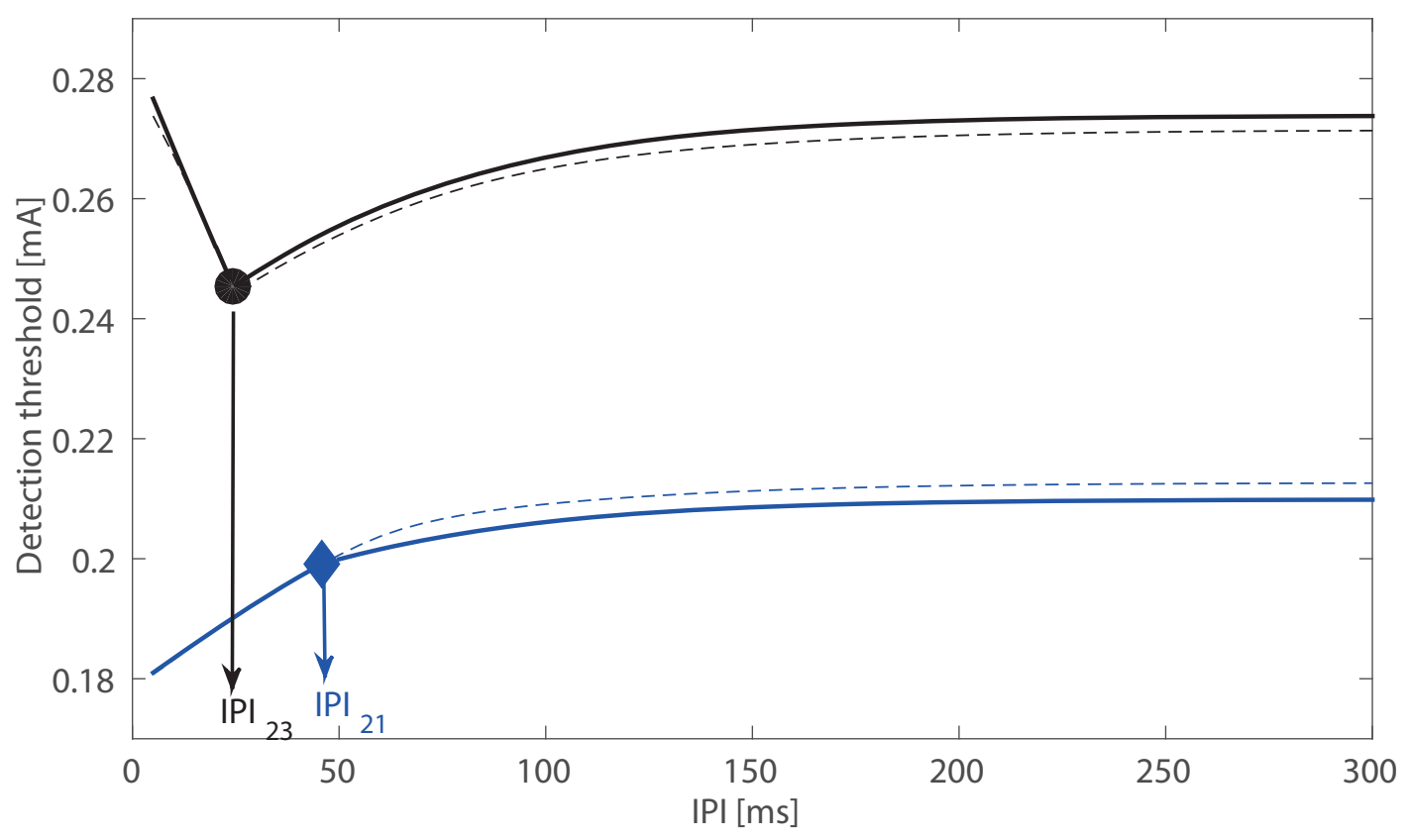

Figure 3.5: Detection threshold as a function of $I P I$. The solid curves for $\tau_{s}, \sigma_{L} \rightarrow 0$ are determined from $\Delta T_{i}, i=1,2,3$. The detection thresholds for nonzero $\sigma_{L}:=8.33 \times$ $10^{-5} \mathrm{~A} / \mathrm{s}, \tau_{s}=1.5 \mathrm{~ms}$ are indicated by dashed curves. For the black curves we use $\tau_{2}=45 \mathrm{~ms}, \lambda_{L}=.01 \mathrm{kHz}$ with product smaller than one. For the blue curves we use $\tau_{2}=45 \mathrm{~ms}, \lambda_{L}=.05 \mathrm{kHz}$ with product larger than one.

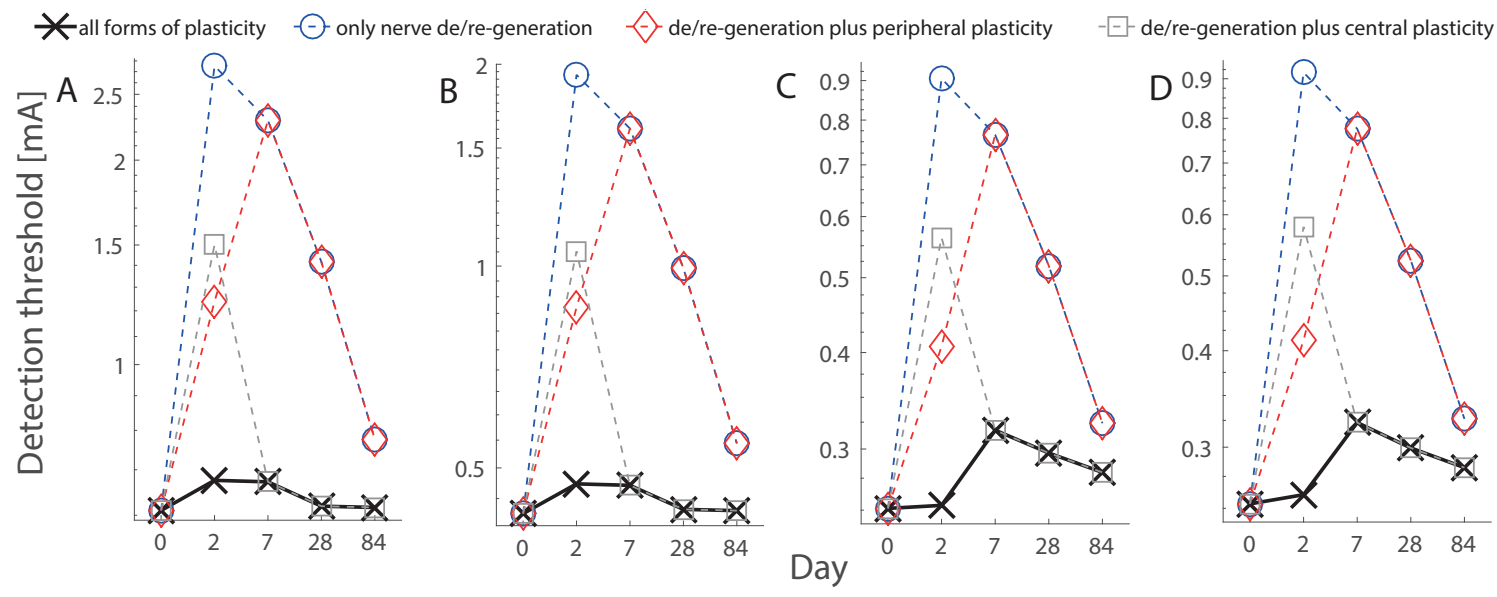

Figure 3.6: Model-based detection thresholds on five study days using four combinations of the temporal properties. We only consider the changes in parameters $\rho, h, V_{t h}, G_{2}$ and $\bar{g}$. Black crosses: all parameter changes included; Blue circles: no functional plasticity; Red diamonds: central functional plasticity omitted; Gray squares: peripheral functional plasticity omitted.

to $50 \mathrm{~ms}$ for double-pulse stimuli, only a small change in the detection thresholds 
was found. This small change could be attributed to a non-monotonic relation between IPI and detection thresholds (Yang et al., 2015a). Our present analysis shows that the product $C_{2} G_{2}^{-1} l \lambda_{h}$ determines the monotonicity. In turn, suppose that the monotonicity can be tested in (future) experimental work, one might treat this monotonicity as an indication to infer changes in $C_{2} G_{2}^{-1} l \lambda_{h}$ as altered central nociceptive properties. We acknowledge that it may be challenging to detect the (non-)monotonicity of detection thresholds, as statistical power may be limited for the IPI from 10 to $50 \mathrm{~ms}$ (Yang et al., 2015a). For future work to verify the existence of non-monotonicity, first, one may use more or other values for the IPI and then study the relation between detection thresholds about these IPIs. Second, one could study the relation between the detection probability and the IPI for double-pulse stimuli by varying IPI but fixing $A$.

\subsubsection{Modeling capsaicin-induced plasticity and its interpre- tation}

We studied plausible neuroplasticity for the topical capsaicin treatment with the computational model. The changes of the fiber densities on study days were set partially based on literature data and also nerve regeneration with a constant regeneration rate. Other changes of physical quantities were based qualitatively on physiological mechanisms (Sandkühler, 2009; Latremoliere and Woolf, 2009; O'Neill et al., 2012). In addition, values of time constants of afferent fibers and secondary neurons were set to similar values obtained in previous experimental studies (Mogyoros et al., 1996; Prescott and Koninck, 2002; Weng et al., 2006). With these physiological restrictions on the computational model, we studied qualitative agreement of simulated patterns of changes of detection thresholds to experimental patterns, see Fig. 3.6. In a more quantitative research, we suggest to estimate the lumped parameters in the model using the stimulus-response pairs of single subjects. The changes in estimates of parameters might unravel the possible individual-specific nociceptive characteristics of capsaicin-induced plasticity.

Capsaicin-induced nerve degeneration is thought to underlie the therapeutic basis in pain management (Anand and Bley, 2011; O'Neill et al., 2012). Our model simulations together with observed elevations of detection thresholds on Day 2, 7 and 28 (Doll et al., 2015c), offer a theoretical support in terms of nociceptive processing. In addition, topical application of capsaicin possibly triggers two forms 
of neuroplasticity pushing single nociceptors or secondary neurons into sensitized states (Coutaux et al., 2005; Sandkühler, 2009; Latremoliere and Woolf, 2009). The two forms of neuroplasticity in peripheral and central nociceptive subsystems compensate the elevations in detection thresholds. Each form of plasticity results in different patterns of changes in detection thresholds using stimuli with single- and double-pulses on the five study days. Our model simulations dissect different longitudinal effects caused by peripheral and central plasticity, as illustrated in Fig. 3.6. Such difference provides valuable information to identify the existence of underlying plasticity.

For our model simulations on Day 2, both forms of peripheral and central plasticity are required for the small change of detection thresholds with $N o P=2$ as well as for clear elevations of detection thresholds with $N o P=1$. Then, the small change in detection threshold with $N o P=2$ is less straightforwardly interpretable. Here, we provide a model-based interpretation based on how physical quantities affect psychometric curves. Nerve degeneration with a clearly decreased value of $\rho$ and increased $h$ tends to shallow $\Psi$ and to shift $\Psi$ to right. However, peripheral plasticity with a decreased value of $V_{t h}$ overcomes this tendency, resulting an effectively smaller shift of $\Psi$ to the left. In addition, central plasticity with lowered $G_{2}$ and increased $\bar{g}$ compensates the dominant tendency with a shallower $\Psi$ caused by the dramatic loss of nerves. Also, it is expected that the psychometric function for double-pulse stimuli is steeper than the counterpart for single-pulse stimuli. Taken together, on Day 2, detection thresholds are elevated for single-pulse stimuli but not for double-pulse stimuli. Using the parameter values of Table 3.1 and 3.2, we show simulation results for the $N o P$-and-day dependent on psychometric functions in Fig. 3.7. In these simulations, we use two combinations $(N o P=1, P W=0.21 \mathrm{~ms})$ and $(N o P=2, I P I=20 \mathrm{~ms}, P W=0.525 \mathrm{~ms})$. The intersection between each psychometric curve and the gray horizontal line is the detection threshold. Psychometric functions with the other two combinations $(N o P=1, P W=0.525 \mathrm{~ms})$ and $(N o P=2, I P I=50 \mathrm{~ms}, P W=0.525 \mathrm{~ms})$ are not shown, as they are quantitatively similar to those in panel $\mathrm{A}$ and $\mathrm{B}$, respectively.

On Day 28, the modeled central neuroplasticity, especially the enhanced synaptic efficiency, compensates the loss of nerve endings, resulting in a less increased detection thresholds for single-pulse stimuli rather than double-pulse stimuli. One can also explain this observation based on the distortion of psychometric functions due to changes in model parameters. As the peripheral plasticity is resolved within 


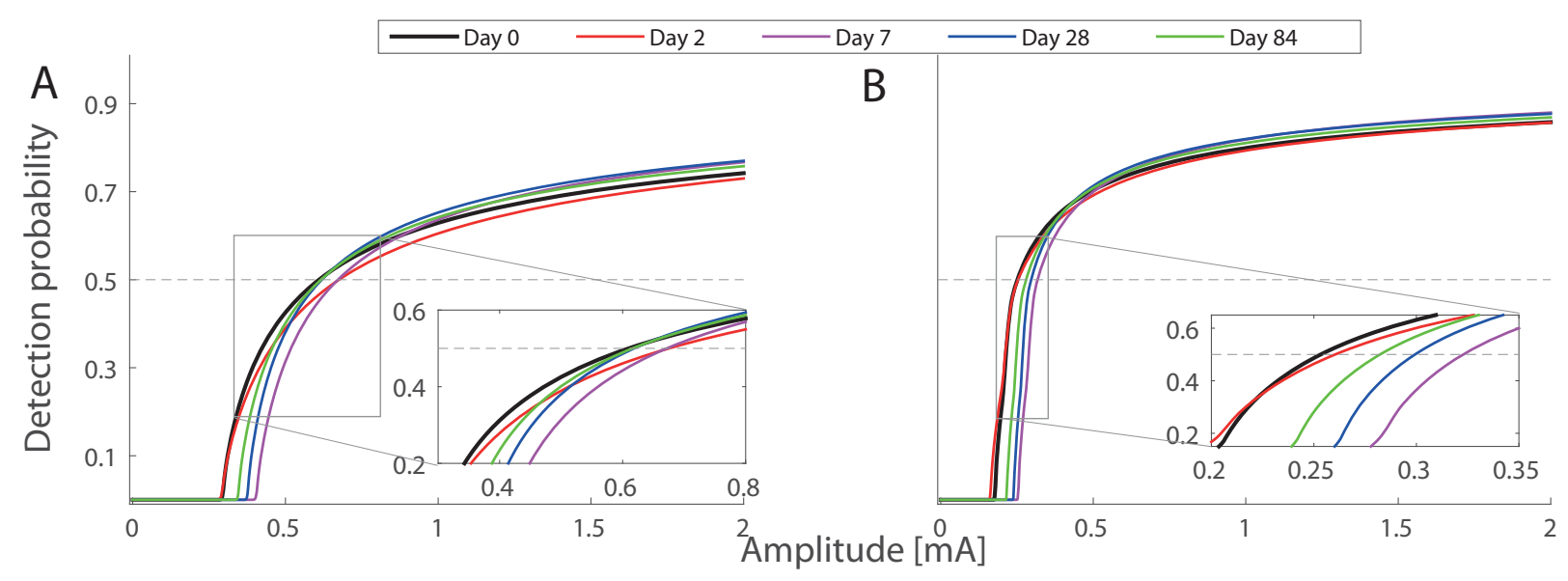

Figure 3.7: Model-based psychometric functions on five study days. The parameter values of the hazard model are set according to Table 3.1 and 3.2. Two combinations of temporal stimulus properties are used in the simulations: A: $(N o P=1, P W=0.21 \mathrm{~ms})$ and B: $(N o P=2, I P I=20 \mathrm{~ms}, P W=0.525 \mathrm{~ms})$. The inset plots show two groups of psychometric functions near detection thresholds.

three days after capsaicin application, $\Psi$ is expected to shift to the right due to an increased value of $h$. In addition, the central plasticity leads to an effectively steeper $\Psi$ than the baseline as nerve endings regenerate with an increased $\rho$ compared to that on Day 2. Hence, all these nociceptive changes result in increases of detection thresholds for double-pulse stimuli, but not for single-pulse stimuli on Day 28. To illustrate this, we show simulations of psychometric functions and the regions near detection thresholds in Fig. 3.7.

\subsubsection{Future studies on capsaicin-induced neuroplasticity}

In our case study, capsaicin-induced central plasticity appears to be present for a much longer time than is usually mentioned for human experimental pain models (Sandkühler, 2009). The time course of such plasticity is similar to estimated time courses found in human subject study using high frequency electrical stimulation as the conditioning stimulus (Pfau et al., 2011). Our results suggest that late-phase synaptic plasticity could have occurred in human subjects. The underlying physiological mechanisms would involve transcription-dependent processes (Kawasaki et al., 2004; Latremoliere and Woolf, 2009). Application of an 8\% capsaicin patch might become a human experimental model to test possible medication to prevent or reverse underlying hypothetical processes of this longer-lasting cen- 
tral plasticity. In addition, our modeling study also sheds light on the design of experiments to test effects of certain medicine on alterations in the nociceptive system. Exploiting the capsaicin-induced plasticity as a human model, one may apply the lidocaine derivative (Binshtok et al., 2007) together to prevent the activation of TTXr-sodium channels over a couple of days. We recommend to compare prospective observations of detection thresholds with our model predictions of detection thresholds in Fig. 3.6. Similar results in such a comparison would further validate our computational model. On the other hand, the possible difference from experimental observations would be useful for further refinement of the model towards more mechanistic insights of capsaicin-induced plasticity. 
4 


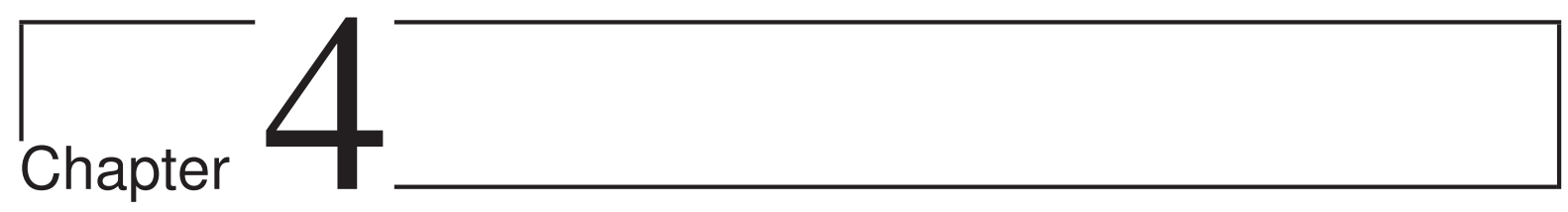

Estimation and identifiability of model parameters in human nociceptive processing using stimulus-response pairs 


\section{Abstract}

Healthy or pathological states of nociceptive subsystems determine different stimulus-response relations measured from quantitative sensory testing. In turn, stimulus-responses measurements may be used to assess these states. In a recently developed computational model, six model parameters characterize activation of A $\delta$ fibers and dorsal horn neurons. However, both model nonlinearity and limited information in stimulus-response pairs challenge to estimate model parameters. Here, we address the question whether and how one can overcome these difficulties for reliable parameter estimation. First, we fit the computational model to experimental stimulus-response pairs by maximizing the likelihood. To evaluate the balance between model fit and complexity, we determine the value of Bayesian Information Criterion. We find that the computational model is better than the conventional logistic model regarding the balance. Second, our theoretical analysis suggests to vary the pulse width among applied stimuli to necessarily prevent structural non-identifiability. In addition, the numerically implemented profile likelihood approach reveals structural and practical non-identifiability. To resolve the practical non-identifiability, we perform a model-based study by choosing values of stimulus properties. Our model-based approach with integration of psychophysical measurements can be useful for a reliable assessment of states of the nociceptive system. 


\subsection{Introduction}

The human nociceptive system provides the neurophysiological basis of pain sensation. Following injury or disease, changes in peripheral and central subsystems could lead to abnormal nociceptive function, e.g. hyperalgesia (Sandkühler, 2009). Long-term alterations in different subsystems can cause persistent pain, reducing quality of life (Voscopoulos and Lema, 2010). Efficient treatments could benefit from improved diagnosis of states of relevant nociceptive subsystems (Woolf and Max, 2001; Wilder-Smith, 2002; Arendt-Nielsen and Curatolo, 2013). Such a differential diagnosis might be achieved by reliably estimating physiologically meaningful parameters of the nociceptive system.

Several experimental paradigms have been proposed to provide information about states of nociceptive subsystems. For example, high throughput technologies can provide rich data about protein expression from pain-related biochemical networks in dissected tissue of animals (Niederberger and Geisslinger, 2008). For clinical practice on human patients, more efforts are still needed to transfer insights from those animal studies. In contrast, psychophysical approaches, e.g. quantitative sensory testing (QST) are non-invasive and can be conducted on human subjects within limited time (Cruz-Almeida and Fillingim, 2014). This makes QST useful for diagnosis of the nociceptive system and its malfunctioning in clinical practice (Walk et al., 2009). However, to dissect contributions of different nociceptive mechanisms, both a well-designed stimulus modality and physiology-based interpretation of limited QST measurements are required.

In general, the human nociceptive pathway first involves peripheral $\mathrm{A} \delta$ and $\mathrm{C}$ nociceptors. Both activated fibers convey the nociceptive information to neurons in the dorsal horn, where resultant activation is transmitted to supraspinal sites. To specifically assess A $\delta$-fiber-mediated nociceptive function, one can apply low-intensity electrocutaneous stimulation with an intra-epidermal needle electrode (Inui et al., 2002; Doll et al., 2014). The use of low amplitudes is compatible with a detection task (Doll et al., 2014; Yang et al., 2015a), where delivered current stimuli are pulse trains characterized by four controllable stimulus properties: the amplitude $(A)$, the number of pulses $(N o P)$, the inter-pulse interval $(I P I)$ and the pulse width $(P W)$. This newly developed experimental paradigm enables to measure various stimulus-response relations by applying stimuli with various values of stimulus properties, collecting a set of stimulus-response pairs (SRPs). This imposes a re- 
quirement to choose their values by considering time scales of peripheral and central peripheral subsystems. First, a single-pulse stimulus with a relatively short $P W$ can recruit $\mathrm{A} \delta$ nociceptors without repetitive recruitment. Varying the $P W$ adjusts the strength of overall peripheral activation (Lapicque, 1907). Changing the stimulus amplitude can also adjust this strength. In contrast to single-pulse stimuli, a pulse-train stimulus introduces temporal summation of the post-synaptic neuronal activity (van der Heide et al., 2009). Furthermore, Gescheider et al. (1999) reported a decreased detection threshold of vibro-tactile stimuli when decreasing IPI. The same authors explained that due to superposition of neural responses. To diagnose sensory function, conventional studies focus on psychophysical characteristics, e.g. detecting hyperalgesia by observing decreased thresholds (Walk et al., 2009). These thresholds are often determined by using a two-coefficient logistic psychometric function, which describes the detection probability with respect to the amplitude with fixed temporal properties (Treutwein, 1995; Doll et al., 2014). Logistic regression is a generalized linear model which yields unique regression coefficients and the detection threshold.

As a generalized linear model, in general, the logistic regression can uniquely determine all regression coefficients and the resultant detection threshold. However, as this model does not straightforwardly account for sensory mechanisms, further efforts are required to interpret these intermediate estimates. In view of a variety of different proposed psychometric curves (Treutwein, 1995), the validity of the logistic curve is questionable, hampering further physiological interpretability of threshold estimates. In addition, more coefficients will be introduced, as the number of different combinations of temporal stimulus properties increases, yielding a potential overfit to a limited number of stimulus-response pairs.

In contrast to this conventional approach, we recently proposed computational models to represent essential mechanisms in both peripheral and central nociceptive subsystems (Yang et al., 2015a). Their states are characterized by six model parameters. The physiologically meaningful interpretation of these parameters offers potential for a mechanism-based diagnosis of the states of nociceptive subsystems. Our previous study demonstrated qualitative agreements between model-based thresholds and experimental thresholds (Yang et al., 2015a). Also, the probabilistic hazard model (HM) is more computationally convenient for parameter estimation. Furthermore, the number of parameters in the HM is constant regardless of the stimulus set, i.e. all combinations of stimulus properties. Fitting a set of SRPs with four 
combinations of temporal parameters, the HM is a simpler model than the logistic regression model with eight regression coefficients. Also, the model-based psychometric function could substantially differ from a symmetric logistic function. However, it has not been studied whether the logistic regression model and the HM have significantly different fits to experimental SRPs.

Given reasonable replication to data by one model, reliable estimation is desired for the purpose of diagnosis and further usage of the parameter estimates. The uncertainty of estimates could be substantial, hampering assessment of the states of nociceptive subsystems. To address the uncertainty, parameter identifiability analysis is essential. Parameter non-identifiability is classified as structural and practical non-identifiability, which have different causes. The former is caused by qualitatively poor input-output measurements, leading to non-unique estimates of model parameters. Equivalently speaking, changes in some non-identifiable parameters can be compensated by changes in other parameters, yielding equally optimal fits to data regardless of measurement accuracy. To analyze structural identifiability, most approaches are based on differential algebra (Ljung and Glad, 1994; Bellu et al., 2007). Given detected structural non-identifiability, one can resolve this by reducing or rebuilding the model. Alternatively, one can enrich types of input-output measurements to eliminate structural non-identifiability. For that, persistence of excitation of subsystems is required for input signals (Miao et al., 2011). In contrast to structural non-identifiability, practical non-identifiability arises from limited information in data contaminated with noise. Hence, approaches based on differential-algebra are not applicable to assess practical identifiability. In contrast, the profile likelihood (PL) approach can be applied to real data to reveal both structural and practical non-identifiability (Raue et al., 2009, 2014).

For the nociceptive detection task, first, no study has evaluated and compared the abilities of both the logistic regression model and the HM to replicate measured SRPs. Model comparison should consider the balance between model fit and complexity, i.e. different numbers of parameters of two models. For this, the Bayesian Information Criterion (BIC) is applicable (Kass and Raftery, 1995; Kingdom and Prins, 2010). Second, parameter identifiability has not been addressed for the nonlinear HM using SRPs from human subjects. To prevent structural non-identifiability, one should formulate prerequisites for the stimulus set. In addition, a limited number of measured SRPs could cause practical non-identifiability for model parameters. A follow-up question is how to resolve non-identifiability by choosing values of stimulus 
properties for additional measurements. The pragmatic PL approach is expected to reveal parameter identifiability for the HM using SRPs.

To address these challenges, we start with a brief description of the experimental data, the logistic regression model and the HM. Next, we optimize their model fits to the data by maximizing the likelihood. Based on the BIC, we assess the balance between model fit and complexity for the HM, and compare this balance with the logistic regression model. After that, we employ the profile likelihood approach to assess parameter identifiability, where some necessary conditions on stimulus sets for structurally identifiable models follow from our theoretical analysis. We perform model-based study to choose the values of stimulus properties for additional measurements. We illustrate that the dataset with both experimentally existing and simulated SRPs can yield improved parameter identifiability.

\subsection{Experiments and models}

We consider a psychophysical experiment with datasets measured from healthy human subjects, focusing on $\mathrm{A} \delta$-fiber-mediated nociceptive subsystems. Then, we outline how we analyze these datasets. Next, we describe the conventional logistic regression model to fit stimulus-response pairs. After that, we briefly introduce the computational model representing both peripheral and central nociceptive subsystems.

\subsubsection{Nociceptive detection task and datasets with stimulus- response pairs}

An single detection experiment lasts 10 minutes, providing around 200 stimulusresponse pairs $\{S, R\}$, where $S$ is the electrocutaneous stimulus and $R$ is a binary response: detected $(R=1)$ or not-detected $(R=0)$. We refer to this collected set of SRPs as an elementary dataset. Stimulus $S$ is delivered by an intra-epidermal needle electrode (Doll et al., 2014). For each applied stimulus, together with the current amplitude $(A)$, three temporal stimulus properties: the number of pulses $\left(N_{o} P\right)$, the interpulse interval $(I P I)$ and the pulse width $(P W)$, determine the pulse train. In each 10-minute experiment, four different combinations of temporal properties were used. For the same combination $(N o P, I P I, P W)$, amplitudes were selected 
according to an adaptive probing procedure (Doll et al., 2014, 2015a). For each combination, the number of SRPs was equal. Depending on whether each temporal property varied among four combinations, there are two designs of stimulus sets: with or without a variation of the $P W$ denoted by $T S_{1}$ and $T S_{2}$, respectively, see Table 2.1. Using these two designs, two different groups of fifteen healthy subjects were

Table 4.1: Two designs with four combinations of temporal stimulus properties for the electrocutaneous pulse-train stimulus. If $N o P=1$, then the $I P I$ is undefined.

\begin{tabular}{cccccc}
\hline Design & Index & $\mathrm{A}$ & $\mathrm{B}$ & $\mathrm{C}$ & $\mathrm{D}$ \\
\hline$T S_{1}$ & NoP $[\#]$ & 1 & 1 & 2 & 2 \\
& $I P I[\mathrm{~ms}]$ & - & - & 10 & 50 \\
& $P W[\mathrm{~ms}]$ & 0.42 & 0.84 & 0.42 & 0.42 \\
\hline$T S_{2}$ & $N o P[\#]$ & 1 & 2 & 2 & 2 \\
& $I P I[\mathrm{~ms}]$ & - & 10 & 50 & 100 \\
& $P W[\mathrm{~ms}]$ & 0.42 & 0.42 & 0.42 & 0.42 \\
\hline
\end{tabular}

recruited, respectively (Doll et al., 2015a). For each subject, the above-described 10-minute detection experiment was conducted on two consecutive days, which we refer to as Day 1 and Day 2.

Here, we briefly describe how we will use these datasets in the following sections. For the design with $T S_{1}$, we consider all available 30 datasets, i.e. 2 for each of 15 subjects, to address the ability how well the logistic regression model and the hazard model replicate data. To address estimation and identifiability of parameters of the HM, we start with 15 elementary datasets measured on Day 1. For the nonlinear HM, estimation and identifiability of parameters could be hampered by the limited amount of information from a single 10-minute experiment. For such cases, we propose criteria to combine datasets from Day 2 with their counterparts of Day 1 for the same subjects for further estimation. For the design with $T S_{2}$, we only use one representative dataset (measured from subject D9001 on Day 1) to demonstrate structural non-identifiability together with a theoretical analysis in the section of identifiability analysis. In total, we consider 31 elementary datasets. 


\subsubsection{The logistic regression model}

By convention, psychophysical studies often employ logistic regression to study the stimulus-response relation Treutwein (1995). With fixed stimulus properties NoP, IPI and $P W$, the detection probability $\operatorname{Pr}(R=1 \mid S)$ is proposed to be a logistic function of the applied amplitude $A$ (Doll et al., 2014). The pair of regression coefficients $\beta_{0}$ and $\beta_{1}$ characterizes the logit transformation as

$$
\operatorname{logit}(\operatorname{Pr}(R=1 \mid S)):=\log \left(\frac{\operatorname{Pr}(R=1 \mid S)}{1-\operatorname{Pr}(R=1 \mid S)}\right)=\beta_{0}+\beta_{1} A .
$$

Considering four combinations of temporal properties in $T S_{1}$, the logistic regression model contains 8 regression coefficients $\boldsymbol{\beta}:=\cup_{j}\left\{\beta_{0}^{j}, \beta_{1}^{j}\right\}$, with $j=1,2,3,4$ corresponding to four combinations A-D of $T S_{1}$ see Table 4.1. Each pair of $\beta_{0}^{j}$ and $\beta_{1}^{j}$ characterizes the logit of the detection probability as

$$
\operatorname{logit}\left(\operatorname{Pr}\left(R=1 \mid S_{j i}\right)\right)=\beta_{0}^{j}+\beta_{1}^{j} A_{j i} .
$$

where $A_{j i}$ is the applied amplitude of the stimulus $S_{j i}$ with the $j$ th combination of temporal stimulus properties. Given a set of stimulus-response pairs, the coefficients can be determined using the GLMFIT routine in MATLAB.

\subsubsection{The hazard model}

We denote the model-based psychometric function, i.e. the conditional probability to detect stimulus $S$, as $\Psi(S):=\operatorname{Pr}(R=1 \mid S)$. A single trial may be simulated by drawing a random number $\xi$ from a standard uniform distribution. The response is $R=1$ when $\xi<\Psi(S)$, indicating that the stimulus is detected, and $R=0$ otherwise.

Here we briefly describe the model, for more details see (Yang et al., 2015a). Peripheral activation by the electrical stimulus is described by the threshold-linear function

$$
\left[f_{A}-\alpha_{1}\right]_{+}:=\pi\left(f_{A}-\alpha_{1}\right) H\left(f_{A}-\alpha_{1}\right)
$$

where

$$
f_{A}:=A\left(1-\exp \left(-\frac{P W}{\tau_{1}}\right)\right),
$$

and $H(\cdot)$ is a Heaviside step function; $\tau_{1}$ and $\alpha_{1}$ are the time constant and the activation threshold of afferent fibers, respectively. Next, through synaptic connections, 
an excitatory post-synaptic current $I_{p}^{*}(t)$ is induced

$$
I_{p}^{*}(t)=\frac{\left[f_{A}-\alpha_{1}\right]_{+}}{\tau_{s}} \sum_{k=0}^{N o P-1} \exp \left(-\frac{t-k I P I}{\tau_{s}}\right) H(t),
$$

with time constant $\tau_{s}=1.5 \mathrm{~ms}$ (Gabbiani et al., 1994). This drives the postsynaptic potential $x(t)$ of a secondary dorsal horn neuron, which we model as a leaky integrator

$$
\tau_{2} \dot{x}=-x+I_{p}^{*}(t), \quad x(0)=0 .
$$

The value of the time constant $\tau_{2}$ is roughly several tens of milliseconds (Prescott and Koninck, 2002; Weng et al., 2006). This noise-free post-synaptic potential is converted into an instantaneous firing rate through a non-homogeneous Poisson process (Plesser and Gerstner, 2000)

$$
\lambda(t)=\lambda_{L}\left(1+\exp \left(\frac{\alpha_{L}-x(t)}{\sigma_{L}}\right)\right)^{-1} .
$$

Here the lumped parameters $\alpha_{L}, \sigma_{L}$ and $\lambda_{L}$ represent the threshold, the slope parameter and the maximal firing rate, respectively. The expected value of the number of spikes during a trial interval of duration $T$ is

$$
\lambda_{T}=\int_{0}^{T} \lambda(t) d t .
$$

The binary response $R$ equals one given sufficient activity in the central nociceptive subsystem. We assume that sufficient activation implies at least one secondary neuron generated an action potential during the trial interval $T$. So the modelbased psychometric function evaluated at the parameters $\boldsymbol{\theta}$ is given by

$$
\Psi_{\boldsymbol{\theta}}=1-\exp \left(-\lambda_{T}\right) .
$$

Our model contains six lumped parameters $\boldsymbol{\theta}=\left(\alpha_{1}, \tau_{1}, \tau_{2}, \alpha_{L}, \sigma_{L}, \lambda_{L}\right)$, which depend on more than ten physical quantities, characterizing peripheral and central nociceptive components. Regarding meaning of lumped parameters, $\alpha_{1}$ and $\tau_{1}$ merely quantify peripheral characteristics, and $\tau_{2}$ and $\lambda_{L}$ merely describe the central properties. But $\alpha_{L}$ and $\sigma_{L}$ are compound characteristics of both subsystems. As known from model development in (Yang et al., 2015a), the obvious functional dependence makes most of physical quantities non-identifiable. Hence in our work, we focus on estimation and identifiability of the lumped parameters rather than physical quantities. 


\subsection{Model fitting and evaluation}

Here, we formulate the likelihood function based on SRPs. We achieve an optimal fit of the HM by maximizing the likelihood function. To evaluate the balance between model fit and complexity, we compute and compare the Bayesian Information Criterion of the HM and the logistic regression model.

\subsubsection{Optimal model fitting of the HM by maximizing the likelihood function}

To quantify the goodness of model fit to SRPs, the likelihood is given by

$$
L(\boldsymbol{\theta})=\operatorname{Pr}(S R P s \mid \boldsymbol{\theta}),
$$

where the SRPs contain a sequence of stimuli and binary responses. The interstimulus interval varied from 2 to 5 sec which is much larger than the time constants in the nociceptive system (Mogyoros et al., 1996; Prescott and Koninck, 2002; Weng et al., 2006). Hence, we assume the trials were independent. Grouping all stimuli with the same stimulus properties, we compute the binomial coefficient from the corresponding responses, and its confidence interval (Clopper and Pearson, 1934).

Together with the fact that same stimulus amplitudes could be utilized multiple times, we represent the measured SRPs as plots between amplitudes and regrouped detection probabilities, see the black dots in Fig. 4.1. The independence among trials simplifies the likelihood (4.9) as

$$
L(\boldsymbol{\theta})=\prod_{k=1}^{N_{S R P}} \Psi_{k}^{R_{k}}\left(1-\Psi_{k}\right)^{1-R_{k}}
$$

where $\Psi_{k}$ is the model-based psychometric function value for the applied stimulus $S_{k}$ evaluated at $\boldsymbol{\theta}$, and $k=1,2, \ldots, N_{S R P}$ with $N_{S R P}$ the total number of stimulus trials. By maximizing the likelihood function, one obtains the optimal values of parameters,

$$
\hat{\boldsymbol{\theta}}=\operatorname{argmax}_{\boldsymbol{\theta} \in \boldsymbol{\Theta}} L(\boldsymbol{\theta})=\operatorname{argmin}_{\boldsymbol{\theta} \in \boldsymbol{\Theta}}-\log L(\boldsymbol{\theta}),
$$

where $\boldsymbol{\Theta}$ is the parameter space. We restrict $\Theta$ to be a hypercubic domain with the lower and upper boundaries of model parameters given in Table 4.2. Our choice of boundaries for $\tau_{1}, \tau_{2}$ and $\lambda_{L}$ is based on experimental studies (Mogyoros et al., 
1996; Prescott and Koninck, 2002; Weng et al., 2006). For the other three, their lower boundaries approach zero. We set the higher boundary of $\alpha_{1}$ to one. We motivate this choice as when $\alpha_{1}>0$, the threshold-linear function (4.3) hardly produces effective activation for the applied amplitudes contrary to the observed dependency of detection probability on amplitudes, see Fig. 4.1. The higher boundaries of $\alpha_{L}$ and $\sigma_{L}$ are set to relatively high values.

Table 4.2: Lower and upper boundaries of the feasible parameter space.

\begin{tabular}{ccccccc}
\hline Model parameter & $\alpha_{1}[\mathrm{~mA}]$ & $\tau_{1}[\mathrm{~ms}]$ & $\tau_{2}[\mathrm{~ms}]$ & $\alpha_{L}[\mathrm{~A} / \mathrm{s}]$ & $\sigma_{L}[\mathrm{~A} / \mathrm{s}]$ & $\lambda_{L}[\mathrm{kHz}]$ \\
\hline Lower boundary & $10^{-6}$ & $10^{-2}$ & 2 & $10^{-5}$ & $10^{-8}$ & $10^{-3}$ \\
\hline Upper boundary & 1 & 3 & $10^{3}$ & 1 & 0.1 & $10^{2}$ \\
\hline
\end{tabular}

Given the nonlinear nature of the HM, multiple local optima of parameters could exist in the landscape of likelihood. To find the global optimum, we employ a Monte Carlo method with multiple starting values. We generate a set of $N_{s}=100$ starting values $\left\{\boldsymbol{\theta}_{\boldsymbol{s}}\right\}$ in $\boldsymbol{\Theta}$ using Latin hypercube sampling (McKay et al., 2000). For each starting value, we use Trust-Region-Reflective-Newton method to obtain the local minimum $\boldsymbol{\theta}_{\boldsymbol{f}}$ (Coleman and $\mathrm{Li}, 1996$ ). After applying this local optimization for all starting values, we take the estimate $\hat{\boldsymbol{\theta}}=\boldsymbol{\theta}_{\boldsymbol{f}}$ with the minimal $-\log L\left(\boldsymbol{\theta}_{\boldsymbol{f}}\right)$ according to (4.11). This numerical optimization is implemented in MATLAB 2014b with the LSQNONLIN routine. Here, we give several remarks regarding optimal model fitting and parameter estimation. First, we acknowledge that this multiple-starting-value optimization scheme with $N_{s}=100$ could not guarantee to obtain the optimal fit, i.e. the lowest $-\log (L)$. To check whether $N_{s}=100$ is sufficiently large, one can sample more starting values, and re-optimize with those samples. If we do not observe any substantially more optimal fit, we consider $N_{s}=100$ sufficient to obtain the optimal fit. In section 4.4, we employ this strategy as a validation procedure by implementing a profile-likelihood approach with more samples.

Second, we clarify two aspects: how well a model replicates observations and how reliable parameter estimates are, although the optimal model fit and parameter estimates are always achieved simultaneously from (4.11). On one hand, plausible models are expected to give a good fit to observations with a relatively small $-\log (\hat{L})$. On the other hand, good replication of data does not imply sufficient identifiability of parameters. For nonlinear models with relatively small $-\log (\hat{L})$, the reliability of parameter estimates could be questionable due to either limited amount of data or an insufficiently rich stimulus set (Raue et al., 2009). We address 
these two aspects separately. We apply the above-mentioned model fitting and parameter estimation to all 31 elementary datasets. With the obtained optimal fitting for SRPs with $T S_{1}$, we address model plausibility in the following subsection. Regarding reliable estimates, the first prerequisite is to obtain estimates $\boldsymbol{\theta}$ within $\boldsymbol{\Theta}$. There are two influential factors: (i) the model structure and (ii) to what extent the observations from a single elementary experiment on Day 1 represent characteristics of nociceptive processing of the subject. In case estimates end up on the boundary of $\Theta$, the model should be refined, which is beyond the scope of this work. Here, for the stimulus set with $T S_{1}$, we try to improve the second factor by combining/adding measurements from the same subject on Day 2, if the two elementary datasets are qualitatively similar. We visually inspect the similarity of the stimulus-response pairs on Day 2 to those on Day 1. For this, we propose two exclusion criteria: either the applied amplitudes were clearly shifted or one or more detection probabilities differed without overlapping confidence intervals. With one combined dataset, we estimate parameters again and check whether they become interior estimates. In case of interior estimates using $T S_{1}$, we further quantify parameter identifiability in section 4.4.

\subsubsection{The balance between model fit and complexity}

During the development of a computational model, one desires to obtain good model fit to experimental observations. In addition, to prevent overfitting with too many free parameters, one needs to reduce the complexity during model development. To assess the balance between model fit and complexity, we propose to use Bayesian Information Criterion (Kingdom and Prins, 2010) given by

$$
B I C=-2 \log (\hat{L})+n_{P} \log \left(n_{D}\right),
$$

where $\hat{L}$ is the optimal likelihood evaluated with parameter estimates $\hat{\boldsymbol{\beta}}$ and $\hat{\boldsymbol{\theta}}$ for the logistic regression model and the HM, respectively. In addition, $n_{D}$ is the number of observations and $n_{P}$ is the number of model parameters. For the logistic regression model (4.2), $n_{P}=8$; for the HM, $n_{P}=6$. The first term of the BIC in (4.12) provides a measure of goodness of fit of the model to stimulus-response pairs. The second term penalizes the BIC for the number of parameters. BIC is a relative measure as the optimal likelihood and $n_{p}$ depend on a specific dataset. Hence, for purposes of model selection given the same dataset, the smaller the value of the BIC is, the more plausible the model is. We compute 30 pairs of BIC values for both 
models with 30 elementary datasets with $T S_{1}$. We check whether HM has a better balance of model fit and complexity with the research hypothesis: the probability that HM has a smaller BIC than the logistic regression model is greater than 0.5. For that, we perform a one-tail binomial test.

\subsubsection{Results}

First, we visualize the fit of the HM and the logistic model to three representative sets of experimental SRPs using $T S_{1}$. We present stimulus-response measurements in Fig. 4.1. The black dots and the dashed curves represent the experimental detection probabilities and their confidence intervals, respectively. The red squares show the predictions of detection probabilities based on $\hat{\boldsymbol{\theta}}$ in the HM. The blue diamonds are the predictions based on the regressed coefficients $\hat{\boldsymbol{\beta}}$ in the logistic regression model (4.2). Row labels A-D correspond to combinations A-D of TS respectively. In Appendix A, we visualize the fitting performance of both models for all 30 datasets using $T S_{1}$.
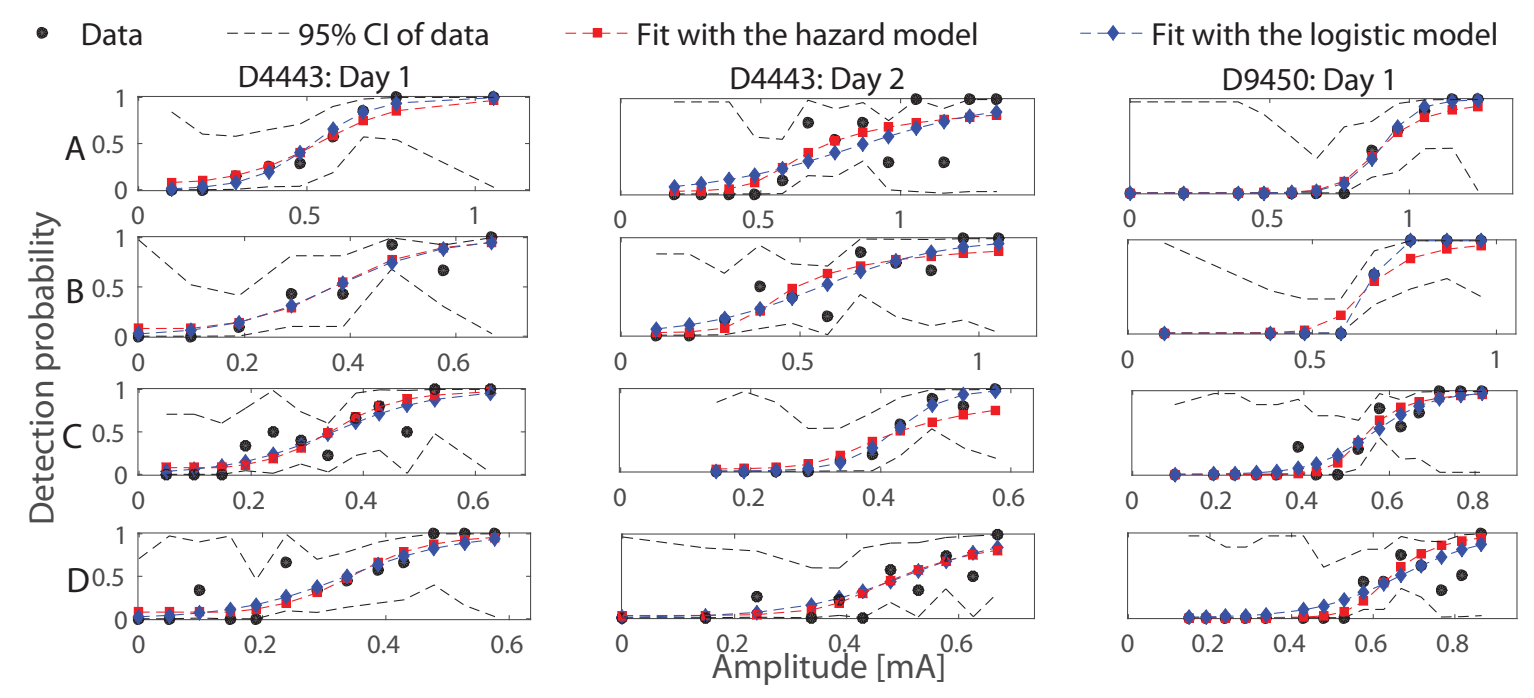

Figure 4.1: Fitting performance of the HM and the logistic model with the optimal values of parameters to three representative sets of experimental stimulus-response pairs using $T S_{1}$, see Table 4.1. The titles of three columns indicate the subject IDs and the measurement day.

Second, Fig. 4.2 shows BIC values of the HM and the logistic model for 30 sets of SRPs from two-day experiments on 15 subjects. There are 24 cases where the $\mathrm{HM}$ has a smaller value of BIC compared to the logistic regression model. We notice that, in most of these 24 cases, the difference of BIC seems small. The binomial 
test yields $p<0.001$, suggesting that the HM is better than the logistic regression model.

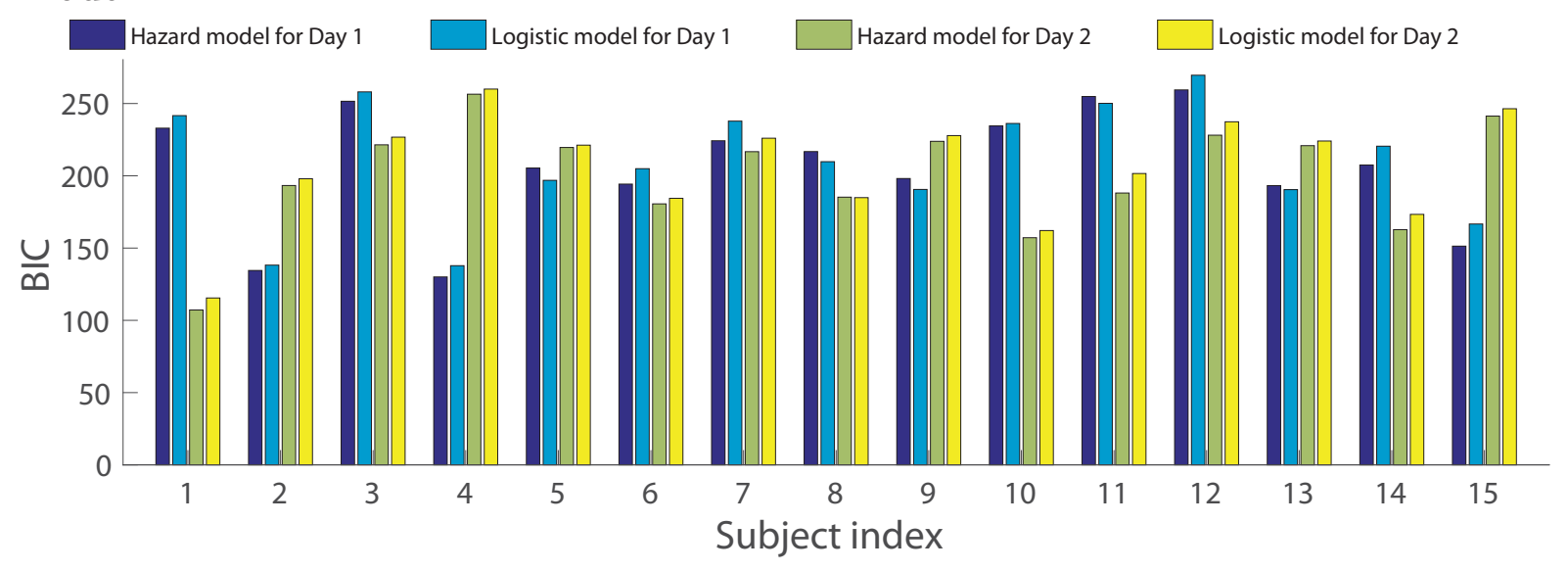

Figure 4.2: Comparison of Bayesian Information Criterion for the HM with those for the logistic model with the measured SRPs on the two study days. Note that each dataset containing about 200 SRPs without combining datasets from the same subject.

Third, for estimation results using SRPs on Day 1, we find that 10 out of 15 estimates lie on the boundary of $\boldsymbol{\Theta}$. After checking the similarity of 10 corresponding pairs of datasets, there are five cases where we can combine the pairs of datasets. For two of these five combined datasets, we obtain interior estimates $\hat{\boldsymbol{\theta}}$. For the other three subjects, even with these additional SRPs, the estimates still lie on the boundary of $\boldsymbol{\Theta}$. Hence, for the design with $T S_{1}$, five elementary datasets on Day 1 and two combined datasets yield interior estimates, for which we will address parameter identifiability.

\subsection{Identifiability analysis}

Even though we obtained reasonable fit of the data to the HM, it is not guaranteed that the estimates of parameters are uniquely determined, i.e. identifiable. Here, we start with a brief description of the profile likelihood approach with emphasis on how it can reveal various types of (non-)identifiability. Next, in case of the hazard model, we perform identifiability analysis in two aspects. First, for structural identifiability, our theoretical analysis helps to formulate a necessary condition on the design of combinations of temporal stimulus properties. Equivalently speaking, we derive a sufficient condition for a structurally non-identifiable HM. In addition, we provide an analytically tractable quantification of the set identifiability of non- 
identifiable parameters, i.e. over which range the structural non-identifiability exists. These findings are illustrated by results from the PL approach with one experimental dataset using $T S_{2}$. Second, to address practical (non-)identifiability, we present the PL results for seven datasets using $T S_{1}$. When practical non-identifiability occurs, we perform a model-based study to efficiently choose stimulus properties for improved parameter identifiability.

\subsubsection{Profile likelihood approach}

The profile likelihood is a univariate function with respect to each single parameter $\boldsymbol{\theta}_{i}$ with $i=1,2, \ldots, n_{p}$,

$$
P L\left(\boldsymbol{\theta}_{i}\right)=\max _{\boldsymbol{\theta}_{\{/ i\}} \in \boldsymbol{\Theta}_{\{/ i\}}} L(\boldsymbol{\theta}),
$$

where $\{/ i\}:=\left\{1,2, \ldots, i-1, i+1, \ldots, n_{p}\right\}$ and $n_{p}$ is the number of parameters (Raue et al., 2009). Fixing one parameter at a specific value $\boldsymbol{\theta}_{i}^{*}$, the value $P L\left(\boldsymbol{\theta}_{i}^{*}\right)$ is determined as the maximal likelihood by tuning the remaining $n_{p}-1$ parameters $\boldsymbol{\theta}_{\{/ i\}}$, i.e.

$$
\hat{\boldsymbol{\theta}}_{\{/ i\}}=\operatorname{argmax}_{\boldsymbol{\theta} \in \boldsymbol{\Theta}} \text { with } \theta_{i}=\theta_{i}^{*} L(\boldsymbol{\theta}) .
$$

The details on the computation of the PL are given in Appendix C. One may notice that the formula of the PL (4.13) is similar to the form to obtain the maximal likelihood (4.11). As more local optimizations are involved, the PL approach can be considered a validation procedure for the obtained estimates and optimal model fit.

Typically, the likelihood is nonzero around the maximum. So, the model with values of parameters around estimates has a positive probability to reproduce the same observation. This results in parameter uncertainty in the parameter space. For identifiability analysis, the PL approach is applicable to assessing uncertainty of parameters of any (non)linear model, provided the likelihood is computable (Raue et al., 2009). Before we directly step to the nonlinear hazard model, we discuss how it works for a conventional linear regression model and then nonlinear models in general.

For linear regression, $-2 \log (P L)$ obeys a quadratic relation with a single regression coefficient. In the sequel, we define $-2 \log (P L)$ as $L P L$. For all coefficients, all parabolas have the same vertical value of vertices $-2 \log (\hat{L})$. The width of the parabola depends on the data quality. In general, increased data length or decreased noise strength will yield a narrower width. The narrower the parabola is, 
the less uncertain the estimate for the single coefficient is. In the extreme case, the parabola will become a horizontal line where the corresponding coefficient is nonidentifiable. To quantify such uncertainty, one can determine confidence intervals of the coefficient due to the fact that $L P L$ approximately follows the $\chi^{2}$ distribution with the degree of freedom equal to one (Uusipaikka, 2008). Given a desired significance level $\alpha$, the confidence intervals of one particular coefficient are given by $\left\{\theta_{i} \mid-2 \log \left(P L\left(\theta_{i}\right)\right) \leq-2 \log (\hat{L})+\chi_{\alpha}^{2}\right\}$. When $\alpha=0.95$, the offset is $\chi_{\alpha}^{2}=3.84$. When $\alpha$ approaches zero, the lower and upper boundaries of the confidence interval coincide to the maximum likelihood estimate. For the extreme situation when $-2 \log (\hat{L})$ is a horizontal line over the entire range of that parameter, confidence intervals are always unbounded for any significance level. Note that this is the only non-identifiable situation for a linear regression model, as the $L P L$ is either a horizontal line or a parabola opening upwards.

For a nonlinear model, $L P L$ could depend in a different way, i.e. not a quadratic relation with respect to a single parameter. When a unique global minimum $L P L$ for one parameter exists, this parameter is structurally identifiable. In contrast, structural non-identifiability manifests itself as multiple different values of $\boldsymbol{\theta}$ with the same optimal fit, diminishing the value of estimate for further use. Those minima may be isolated in the parameter space for a nonlinear model. However, in general situations, multiple global minima form some manifold in the parameter space. As a result, $L P L$ plots of some parameters will be flat. In case that such a manifold extends to boundaries of some parameters, the flatness in corresponding $L P L$ plots will span the entire range of parameters. In other cases, the flatness of the $L P L$ plot spans a narrower interval for the corresponding parameter. For this specific kind of structural non-identifiability, the concept of set identifiability is useful, i.e. a set of multiple estimates have the minimal LPL over a restricted range (Schmidt, 2015). To determine the range can help to understand the specific parameter identifiability. Given the model structure, one can study the structural identifiability. Several studies derived sufficient or necessary conditions for structurally identifiable for models with specific structures (Gargash and Mital, 1980; Saccomani and Cobelli, 1993; Bazanella et al., 2012). However, no universally sufficient-and-necessary conditions have been formulated for an arbitrary nonlinear model. On one hand, sufficient conditions can guarantee model identifiability, but such requirements could be too strict to be met in practice (Bellman and Åström, 1970; Chiş et al., 2011). On the other hand, finding necessary conditions prevents to measure input-output relations with in-sufficient excitation of subsystems, which is helpful before conducting expensive 
experiments, e.g. human subject experiments.

Next, for structurally identifiable parameters, practical non-identifiability could arise from the limited amount of information from existing observations. Similar to the linear case, one can define the confidence interval as

$$
C I_{\alpha}\left(\theta_{i}\right)=\left\{\theta_{i} \in\left(\theta_{i}^{l}, \theta_{i}^{u}\right) \mid-2 \log \left(P L\left(\theta_{i}\right)\right) \leq-2 \log (\hat{L})+\chi_{\alpha}^{2}\right\},
$$

where $\theta_{i}^{l}, \theta_{i}^{u}$ are the lower and upper boundaries of parameter $\theta_{i}$, respectively. When the significance level $\alpha \rightarrow 0$, the confidence interval becomes just the maximumlikelihood estimate. However, by increasing $\alpha$, the corresponding confidence interval could become unbounded when $\alpha=\alpha^{*}$. For this, we define that the parameter can only be practically identifiable below the critical significance level $\alpha^{*}$, i.e. at least one boundary of the confidence interval is identical to one boundary of the parameter space. To address practical identifiability, we need to treat each dataset case by case, because the PL reflects the likelihood landscape.

\subsubsection{Structural and practical non-identifiability in the HM}

In the case of the nonlinear HM, structural and practical non-identifiability could occur. First, structural non-identifiability could arise from the qualitatively insufficient stimulus set, i.e. $(A, N o P, I P I, P W)$ rather than observed stimulusresponse pairs contaminated with noise. Such insufficiency results in non-unique parameter estimates. As one trivial example, when only amplitudes below the peripheral activation $\alpha_{1}$ are applied, this implies $A\left(1-\exp \left(\frac{P W}{\tau_{1}}\right)\right)<\alpha_{1}$ with any value of $P W$. No matter how combinations of temporal stimulus properties are chosen, one can expect that $\alpha_{1}$ is structurally non-identifiable due to below-threshold activation in (4.3). Hence, a prerequisite for a structurally identifiable HM is to apply some amplitudes above $\alpha_{1}$. In addition to this trivial case, in section 4.4.2.1, we perform an analytical analysis to provide one sufficient condition causing structural non-identifiability on the $P W$. As a further step, we quantify the set identifiability for such structurally non-identifiable case. To illustrate the structural non-identifiability, we show the PL results using one experimental dataset in section 4.4.3.1. Second, as practical identifiability depends data quality, we perform the PL approach with seven cases with interior estimates using $T S_{1}$, with results in section 4.4.3.1. In case of detected practical non-identifiability, we aim to efficiently resolve that by providing additional SRPs. For this, we propose a model-based method to 
choose stimulus properties in section 4.4.2.2, and show the results with improved identifiability in section 4.4.3.2.

\subsubsection{Theoretical analysis for structural non-identifiability in the HM}

In detection task with pulse-train stimuli, we use a relatively large IPI ( $\geq$ $10 \mathrm{~ms}$ ) compared to the time constant for peripheral activation (Mogyoros et al., 1996). Then the $P W$ is the only effective temporal property to control peripheral activation. In case that the $P W$ is invariant for all stimuli, changes of parameters for central processing may be compensated by other parameter changes for peripheral activation. This hampers separation of their contributions to the overall nociceptive function, leading to non-identifiable parameters. Such a suspicion deserves a rigorous study for structural identifiability. There are two questions: whether a stimulus set with identical $P W$ definitely introduces structurally non-identifiable parameters. If so, how to quantify the set identifiability for resultant structurally non-identifiable parameters? To answer the first, we perform a theoretical analysis with the hazard model. Our strategy is to search for a redundant quantity with respect to $\boldsymbol{\theta}$. The cascaded structure in the HM facilitates our search. By investigating peripheral activation (4.3) together with synaptic transmission (4.5), and processing and activation of secondary neurons $(4.6,4.7)$, one can find that redundancy of $\boldsymbol{\theta}$ exists in the instantaneous firing rate for secondary neurons $\lambda(t)$. For example, given two vectors of different parameter values $\boldsymbol{\theta}^{(1)}$ and $\boldsymbol{\theta}^{(2)}$ satisfying

$$
\left\{\begin{aligned}
\frac{\alpha_{1}^{(1)}}{\sigma_{L}^{(1)}} & =\frac{\alpha_{1}^{(2)}}{\sigma_{L}^{(2)}}, \\
\frac{\alpha_{L}^{(1)}}{\sigma_{L}^{(1)}} & =\frac{\alpha_{L}^{(2)}}{\sigma_{L}^{(2)}}, \\
\frac{1-\exp \left(-\frac{P W}{\tau_{1}^{(1)}}\right)}{\sigma_{L}^{(1)}} & =\frac{1-\exp \left(-\frac{P W}{\tau_{1}^{(2)}}\right)}{\sigma_{L}^{(2)}}, \\
\tau_{2}^{(1)}=\tau_{2}^{(2)} & , \lambda_{L}^{(1)}=\lambda_{L}^{(2)},
\end{aligned}\right.
$$

the HM can produce the same $\lambda(t)$ given any arbitrary values of $t, N o P$ and $I P I$. Given this, all quantities depending on $\lambda(t)$, including the likelihood function (4.10), become redundant. Suppose that one obtains $\hat{\boldsymbol{\theta}}$ from the numerical optimization in section 4.3, a distinct parameter vector $\boldsymbol{\theta}^{*}=\left(\alpha_{1}^{*}, \tau_{1}^{*}, \tau_{2}^{*}, \alpha_{L}^{*}, \sigma_{L}^{*}, \lambda_{L}^{*}\right)$ with two following restrictions, which are rewritten from (4.16): (i) $\tau_{2}^{*}=\hat{\tau_{2}}, \lambda_{L}^{*}=\hat{\lambda_{L}}$ and (ii)

$$
\frac{\alpha_{1}^{*}}{\hat{\alpha_{1}}}=\frac{\alpha_{L}^{*}}{\hat{\alpha_{L}}}=\frac{\sigma_{L}^{*}}{\hat{\sigma_{L}}}=\frac{1-\exp \left(-\frac{P W}{\tau_{1}^{*}}\right)}{1-\exp \left(-\frac{P W}{\tau_{1}}\right)},
$$


will have the same optimal fit. The restriction (i) implies that the parameters $\tau_{2}$ and $\lambda_{L}$ could be structurally identifiable. However, four parameters $\alpha_{1}, \alpha_{L}, \sigma_{L}$ and $\tau_{1}$ in (ii) are structurally non-identifiable.

With detected structural non-identifiability, we investigate whether and how the restriction (ii) can affect the set identifiability. By denoting

$$
r^{*}:=\frac{1-\exp \left(-\frac{P W}{\tau_{1}^{*}}\right)}{1-\exp \left(-\frac{P W}{\tau_{1}}\right)}
$$

we obtain $\alpha_{1}^{*}=r^{*} \hat{\alpha_{1}}, \alpha_{L}^{*}=r^{*} \hat{\alpha_{L}}$ and $\sigma_{L}^{*}=r^{*} \hat{\sigma_{L}}$ according to (4.17). Given obtained $\hat{\boldsymbol{\theta}}, \alpha_{1}^{*}, \alpha_{L}^{*}$ and $\sigma_{L}^{*}$ depend on $r^{*}$. One can find that $r^{*}$ is a decreasing function of $\tau_{1}^{*} \in\left(\tau_{1}^{l}, \tau_{1}^{u}\right)$. Hence, one has $r^{*} \in\left(r^{l}, r^{u}\right)$, where $r^{l}=\frac{1-\exp \left(-\frac{P W}{\tau_{1}^{u}}\right)}{1-\exp \left(-\frac{P W}{\tau_{1}}\right)}$ and $r^{u}=\frac{1-\exp \left(-\frac{P W}{\tau_{1}^{l}}\right)}{1-\exp \left(-\frac{P W}{\tau_{1}}\right)}$. To quantify the set identifiability, one use the ratio between boundaries of $r^{*}$ given by

$$
r:=\frac{r^{u}}{r^{l}}=\frac{1-\exp \left(-\frac{P W}{\tau_{1}^{l}}\right)}{1-\exp \left(-\frac{P W}{\tau_{1}^{u}}\right)}
$$

With $P W=0.42 \mathrm{~ms}, \tau_{1}^{l}=0.01 \mathrm{~ms}$ and $\tau_{1}^{u}=3 \mathrm{~ms}$ in Table 4.2, we obtain $r=7.6545$. However, we expect the ratio of the boundaries for $\tau_{1}$ is $3 / 0.01=300$, i.e. nonidentifiability spanning the entire range. To illustrate our theoretical analysis, we employ the profile likelihood approach to the elementary dataset from subject D9001 on Day 1 using the setting $T S_{2}$. We expect flatness to exist in the $L P L$ plot with respect to each of those four parameters. For $\alpha_{1}, \alpha_{L}$ and $\sigma_{L}$, the flatness should only span a finite interval with the ratio of the range of 7.6545 .

\subsubsection{Choice of stimulus properties to improve practical identifiability}

An existing dataset may cause practically non-identifiable parameters with unbounded confidence intervals. For each parameter, the PL implicitly defines a curve $\boldsymbol{c}_{i}$ in parameter space parameterized by $\boldsymbol{\theta}_{i}$ and corresponding $\hat{\boldsymbol{\theta}}_{\{/ i\}}$ within the $95 \%$ confidence interval according to (4.14) and (4.15). For all six parameters, we denote the parameter set $\Theta^{E}:=\cup_{i=1}^{6} \boldsymbol{c}_{i}$, which can produce similarly good fits to the existing SRPs compared to that based on estimates $\hat{\theta}$. Here, we want to study whether additional stimulus-response pairs can improve parameter identifiability, i.e. a narrower confidence interval. 
First, for an efficient improvement, we perform a model-based study to choose values $S^{E}:=(A, N o P, I P I, P W)$. These values are not necessarily identical to any already used one. For this, we assume feasible values of amplitudes from 0 to 2 by a step value of $0.1 \mathrm{~mA}$. We only consider single and double pulse stimuli with the $P W$ from 0.105 to 1.05 by a step $0.105 \mathrm{~ms}$. Regarding the $I P I$, we set its values from 10 to 150 by a step value of 10 ms. Given additional psychophysical SRPs, one can expect that the width of confidence intervals depends on the variability among the psychometric function (4.8) for $S^{E}$ simulated with parameter values from the set $\boldsymbol{\Theta}^{E}$. This impact can be quantified by the variance of the predicted values of $\Psi_{\boldsymbol{\theta}}$

$$
V\left(S^{E}\right):=\int_{\Theta^{E}}\left(\Psi_{\boldsymbol{\theta}}\left(S^{E}\right)-\bar{\Psi}\left(S^{E}\right)\right)^{2} p(\boldsymbol{\theta}) d \boldsymbol{\theta}
$$

where the expected value $\bar{\Psi}\left(S^{E}\right):=\int_{\boldsymbol{\Theta}^{E}} \Psi_{\boldsymbol{\theta}}\left(S^{E}\right) p(\boldsymbol{\theta}) d \boldsymbol{\theta}$ and $p(\boldsymbol{\theta})$ is the probability density function of $\boldsymbol{\theta}$. The larger the value of $V\left(S^{E}\right)$ is, the more distinguishable parameter values among $\boldsymbol{\Theta}^{E}$ are. Equivalently speaking, one can expect a larger influence of additional stimuli with these properties on the likelihood landscape. Our metric, $V\left(S^{E}\right)$, is similar to that utilized in the model-based experimental planning to improve parameter identifiability for gene regulatory mechanisms (Steiert et al., 2012). To calculate (4.20), as six model parameters have different physical units, we assume that each parameter uniformly distributes in the logarithmic scale. We approximate (4.20) by numerically discretizing $\boldsymbol{c}_{i}$ on a logarithmic scale with a step $\log (1.001)$. To most efficiently distinguish values of parameters within $\Theta^{E}$, we find the $S^{O}$ with the largest variance

$$
S^{O}:=\operatorname{argmax}_{S^{E} \in \Omega} V\left(S^{E}\right)
$$

where $\Omega$ contains all feasible values of stimulus properties. We determine $S^{O}$ in (4.21) by the method of exhaustion.

Second, to check effect of additional SRPs with the choice of $S^{O}$ on parameter identifiability, we perform a validation study. We generate 100 additional responses to the stimulus with $S^{O}$, following a binomial distribution with the probability $\Psi_{\boldsymbol{\theta}}\left(S^{O}\right)$ evaluated at $\hat{\boldsymbol{\theta}}$. Both these additional SRPs and existing data form a hypothetical dataset. Using this dataset, we re-perform parameter estimation and identifiability analysis. We compare the resultant profile likelihood with the original PL from the dataset merely containing experimental SRPs. 


\subsubsection{Results}

Applying the PL approach, we present the possible types of parameter identifiability from the existing experimental datasets. For one practically non-identifiable case, we demonstrate our model-based study to improve parameter identifiability.

\subsubsection{Profile likelihood}

First, to validate results of optimal fits and estimates obtained in section 4.3, we perform the PL approach to the 15 elementary datasets on Day 1 using $T S_{1}$. The PL approach gives identical estimates and optimal fit for 12 datasets. For the other three cases, we find negligible difference in optimal fits, i.e. $-2 \log (\hat{L})$, see details in Appendix B. In addition, qualitatively the estimates are similar, i.e. estimates from the PL approach still stay on the boundary of $\Theta$ similar to those from the multiple-starting-value scheme. This validates our usage of datasets for both model comparison and identifiability analysis.

Second, to illustrate the structural non-identifiability, we show $L P L$ for subject D9001 using the setting $T S_{2}$ in Fig. 4.3. The $95 \%$ confidence interval of each parameter is indicated by the region below the horizontal dashed line with the offset $\chi_{95}^{2}=3.84$, see Fig. 4.3A-E. As we expect from (4.17), the flatness of $L P L$ at the minimal value occurs for parameters $\alpha_{1}, \tau_{1}, \alpha_{L}$ and $\sigma_{L}$. For $\tau_{1}$, the flatness spans its entire range. On the other hand, for $\alpha_{1}, \alpha_{L}$ and $\sigma_{L}$, the flatness of $L P L$ at the minimum only spans a shorter interval. The ratio between the upper and lower boundaries of this interval is about 7.6545. This agrees with the computed value from (4.19).

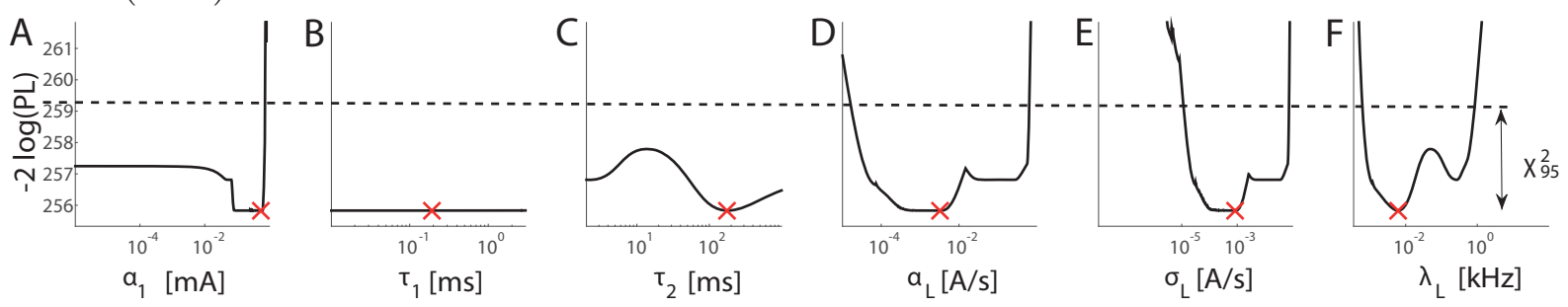

Figure 4.3: Profile likelihood plot for the structurally non-identifiable case using the dataset containing about 200 SRPs using the setting $T S_{2}$ from subject D9001. Red crosses indicate the estimated values of parameters from the multiple-starting value optimization. The horizontal dashed line marks $95 \%$ confidence intervals.

Third, for the identifiable case, we show $-2 \log (P L)$ for subject D9450, see Fig. 
4.4. The intersections of $-2 \log (P L)$ with the dashed horizontal line are the bounds of the $95 \%$ confidence interval. All six model parameters are practically identifiable at least up to the $95 \%$ confidence level.

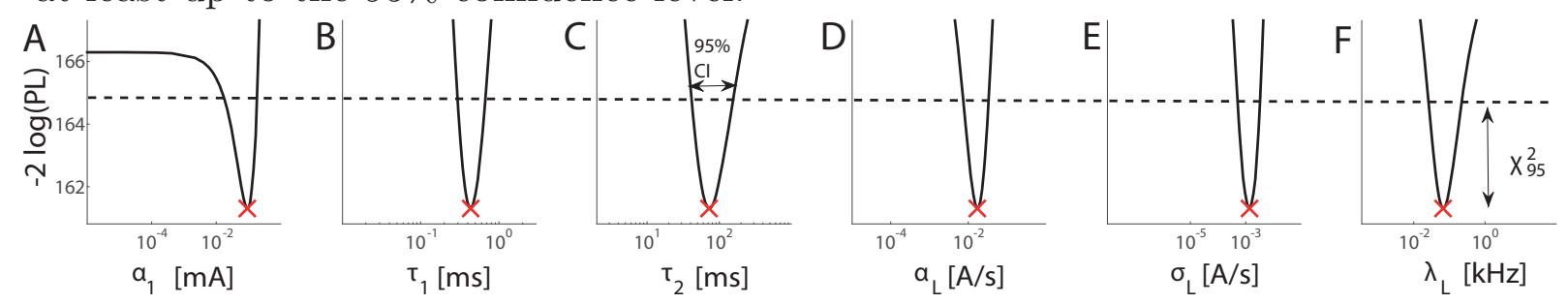

Figure 4.4: Profile likelihood plot for subject D9450 with about 200 SRPs using the setting $T S_{1}$. Red crosses indicate the estimated values of parameters from the multiple-starting value optimization. The horizontal dashed line marks $95 \%$ confidence intervals.

Fourth, we give an example of practical non-identifiability with the combined dataset from subject D4443, see Fig. 4.5. The $L P L$ extends to the lower boundary of $\alpha_{1}$, indicating severe practical non-identifiability. Given the unbounded $95 \%$ confidence intervals of $\tau_{2}$, the time constant $\tau_{2}$ is also practically non-identifiable. In addition, we detect that profile likelihoods of the parameters $\alpha_{L}, \sigma_{L}$ or $\lambda_{L}$ have multiple local minima. Taken together, it indicates that the existing experimental data is insufficient to constrain model parameters. More measurements are required to further constrain towards narrower confidence intervals. We will continue the model-based study for this case in section 4.4.3.2.

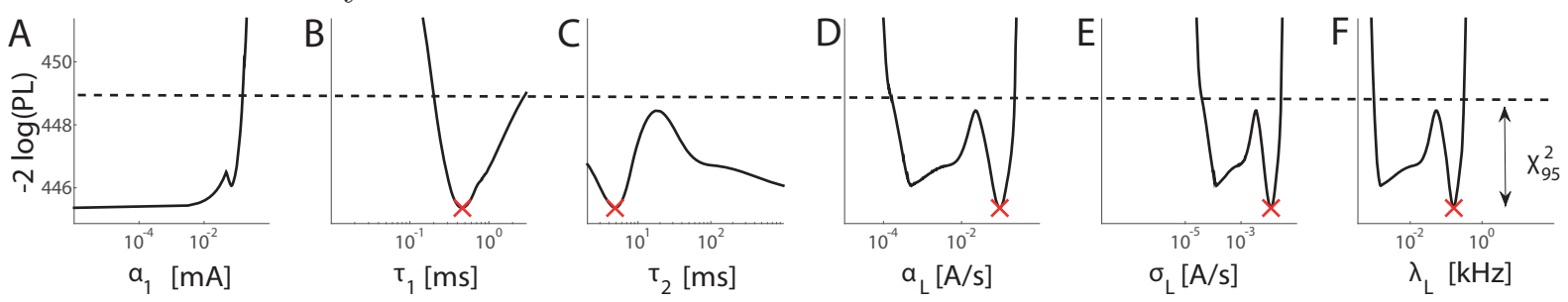

Figure 4.5: Profile likelihood results with about 400 SRPs measured from two-consecutiveday measurements on the subject D4443 using the setting $T S_{1}$. Red crosses indicate the estimated values of parameters from the multiple-starting value optimization. The horizontal dashed line marks $95 \%$ confidence intervals.

For the design of $T S_{1}$, we summarize PL results for four other elementary datasets and one combined dataset with interior estimates in Appendix D. We notice that there is a case with severe practical non-identifiability for $\alpha_{1}$ with SRPs from subject D4443, shown in Fig. 4.5A. In contrast to the stimulus set with $T S_{2}$, we did not observe that a single parameter was severely non-identifiable for all the seven 
cases. This indicates that a stimulus set with all stimulus properties varied, e.g. $T S_{1}$, may achieve the structural identifiability.

\subsubsection{Choice of stimulus properties to improve practical identifiability}

To resolve practical non-identifiability, as observed in Fig. 4.5, we perform model-based experimental design with the set $\Theta^{E}$. In Fig. 4.6, we show the contour plots of $V\left(S^{E}\right)$ for varying $A$ and $P W$. For $I P I>30 \mathrm{~ms}$, the contour plots (not shown) are qualitatively similar to Fig. 4.6D. The optimal choice $S^{O}$ is $(N o P=2$, $I P I=20 \mathrm{~ms}, P W=1.05 \mathrm{~ms}, A=0.2 \mathrm{~mA}$ ) with the largest $V\left(S^{E}\right)$, see Fig. $4.6 \mathrm{C}$.

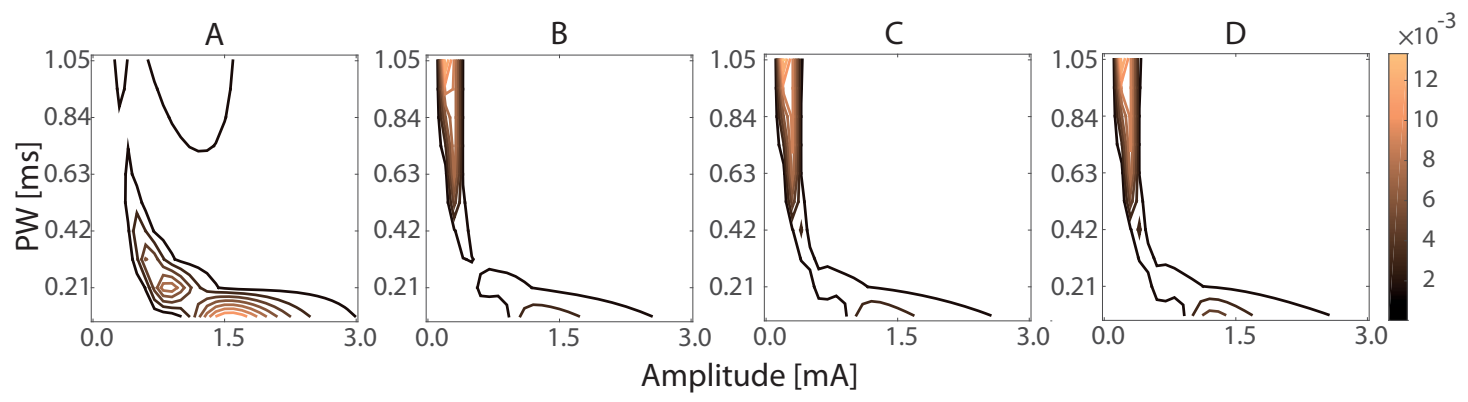

Figure 4.6: Contour plots of the variance $V\left(S^{E}\right)$ for varying $A$ and $P W$ for fixed $N o P$ and IPI. A: $N o P=1, \mathrm{~B}: N o P=2, I P I=10 \mathrm{~ms}, \mathrm{C}: N o P=2, I P I=20 \mathrm{~ms}, \mathrm{D}: N o P=2$, $I P I=30 \mathrm{~ms}$.

With the aim to get narrower confidence intervals, we set the stimulus properties as $S^{O}$. In our validation, we generate additional SRPs by using $\boldsymbol{\theta}=\left(\alpha_{1}=1.001 \times\right.$ $\left.10^{-6}, \tau_{1}=0.46, \tau_{2}=4.83, \alpha_{L}=0.095, \sigma_{L}=0.012, \lambda_{L}=0.16\right)$ estimated from the existing combined dataset from subject D4443. Fig. 4.7 presents results of the profile likelihood (in red) using the hypothetical dataset containing both existing experimental SRPs and additionally simulated 100 SRPs. The hypothetical dataset yields narrower confidence intervals for each model parameter than original ones (in black). For parameters $\alpha_{L}, \sigma_{L}$ and $\lambda_{L}$, the global minimum in the $L P L$ becomes more obvious in Fig. 4.5D, E and F, respectively. In Fig. 4.7A, we notice that for this subject the $L P L$ of $\alpha_{1}$ still extends to its negative direction, suggesting that $\alpha_{1}$ is still non-identifiable. 


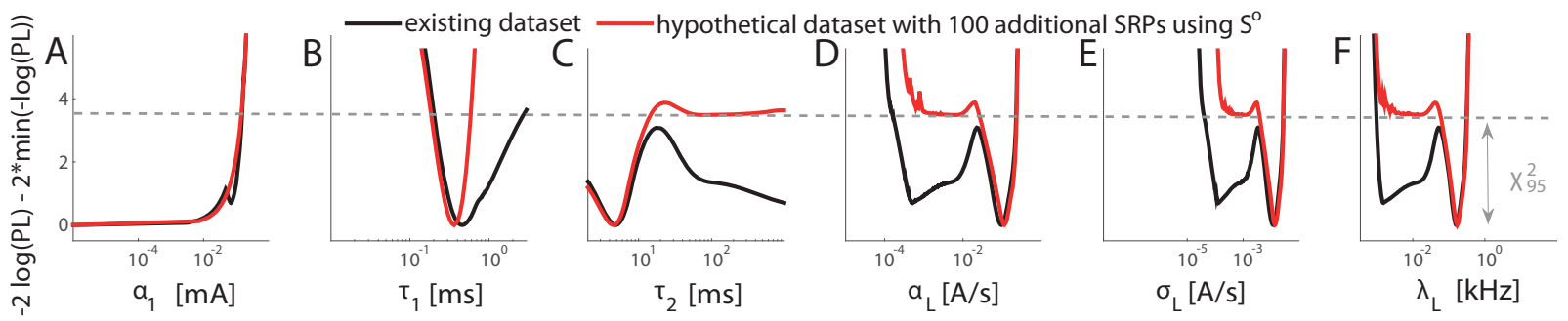

Figure 4.7: Comparison results of parameter identifiability from the PL approach. The y-axis was shifted for visualization purposes.

\subsection{Discussion}

In our study, we integrated experimental data with the hazard model for parameter estimation and identifiability of physiological parameters of human nociceptive processing. We obtained good fits with the HM to SRPs. Based on BIC, comparison with the logistic regression model suggested a better balance between fit and complexity for the HM. By applying the profile likelihood approach, we demonstrated that it was possible to achieve parameter identifiability using 10-minutes measurements containing about 200 stimulus-response pairs, but not always. For structurally identifiability, our theoretical analysis provided a necessary condition. To resolve detected practical non-identifiability, we demonstrated our model-based method in choosing values of stimulus properties for an improved identifiability.

In conventional psychophysical studies, stimulus-response pairs are usually fitted to logistic and similar models (Treutwein, 1995). There are few studies to estimate physiology-based model parameters using psychophysical data, see e.g. (Alcalá-Quintana and García-Pérez, 2013). To the best of our knowledge, our study is the first to demonstrate the estimation of multiple model parameters characterizing peripheral and central nociceptive subsystems using psychophysical SRPs. In addition, our study demonstrates the applicability of the PL approach with stimulusresponse measurements and its merit to assess both structural and practical identifiability.

In this study, we compared the BIC values from the two models using datasets with $T S_{1}$. Although, in 24 out of 30 cases, the HM had smaller BICs than those from the logistic regression model, we notice this may be attributed to the difference in the model complexity rather than the difference in model fits, see Fig. 4.2. This indicates that the fit by the logistic regression model to data is not worse than that from the HM, although the logistic regression model does not explicitly represent 
nociceptive mechanisms. Our present study did not present the BIC of the HM using a group of datasets with $T S_{2}$. Given structural non-identifiability shown in Fig. 4.3, via model reduction by fixing $\tau_{1}$ to a constant value, one can expect that the reduced model is still able to have the same optimal fit $-2 \log (\hat{L})$ as the sixparameter hazard model. For the (reduced) HM, the BIC value will further decrease, leading to a better balance between model fit and complexity.

Typically, for dynamical models representing biochemical processes, PL plots of structurally non-identifiable parameters were flat for the entire interval, which is restricted by lower and upper pre-defined boundaries (Raue et al., 2009). Recent work by Schmidt (2015) suggested to use set identifiability to quantify the range of non-identifiability, when structurally non-identifiable parameters span a restricted range. In our identifiability analysis of the hazard model with six parameters, first, we show that a stimulus set with invariant $P W$ results in four structurally nonidentifiable parameters $\alpha_{1}, \tau_{1}, \alpha_{L}$, and $\sigma_{L}$. Second, we formulated the ratio $r$ in (4.19) to quantify the range of set identifiability in an analytically tractable way. This ratio depends on the boundaries of $\tau_{1}$ and temporal property $P W$. Its upper boundary has a larger impact on the ratio than the lower one. On the other hand, when the $P W$ is not varied in the stimulus set, the larger $P W$ is, the smaller $r$ is, see Appendix E. It implies that saturation of activation of $\mathrm{A} \delta$ fibers helps to distinguish different nociceptive mechanisms with a smaller interval of set identifiability for nonidentifiable $\alpha_{1}, \alpha_{L}$, and $\sigma_{L}$. Together with prevention of repetitive recruitment of the same nerve endings during one pulse, we suggest to use a value of $P W$ like $1.05 \mathrm{~ms}$. In addition to these analytical arguments, the PL approach can further determine the boundary values of set identifiability for $\alpha_{1}, \alpha_{L}$, and $\sigma_{L}$ given experimental SRPs, see Fig. 4.3A, D, and E.

Mechanism-based diagnosis of (mal)functioning of the nociceptive system can benefit from our developed approaches on parameter estimation and identifiability analysis. In pain research, various experimental pain models have been developed to perturb the nociceptive system (Szallasi, 2010). For example, high frequency electrical stimulation is known to induce central sensitization. Further validation studies would be useful to test our quantitative approaches with psychophysical datasets measured from sequential phases in experimental pain models. In clinical practice, diseases or medical intervention could result in neuroplasticity, accompanying with (mal)adaptive behavior. With our approaches, in turn, new insights of responsive mechanisms underlying nociceptive malfunctioning could be gained by monitoring 
changes in estimates of parameters over time.

We performed a model-based study to improve parameter identifiability. We verified the improvement by using a hypothetical dataset with both existing and additionally model-simulated SRPs, illustrated by narrower confidence intervals. Here, we discuss two issues. First, we notice that $\alpha_{1}$ seems to be still practically non-identifiable in the direction to its lower boundary. On one hand, such non-identifiability may be caused by the specific properties of afferent fibers of this subject D4443. One possible explanation is that tips of free nerve endings are close to the skin, resulting in such a low value of $\alpha_{1}$. On the other hand, the obtained PL also suggests that $\alpha_{1}$ is unlikely to be higher than $0.13 \mathrm{~mA}$ given the $95 \%$ confidence level. Second, regarding the practical implementation of the measurements of SRPs with the optimal choice of stimulus properties $S^{O}$, we acknowledge that one should not stimulate the subject repetitively with an identical stimulus to avoid anticipation by the subject. One may interleave the designed stimulus with those used earlier in some random manner (Doll et al., 2014). For example, one can perform a 10-minute experiment with 50\%-50\% sampling weights for the optimal and other values of stimulus properties, respectively. This can result in about 100 stimulusresponse pairs with $S^{O}$. Given the simulated dataset, we have shown the improved identifiability in Fig. 4.7. We expect that the parameter identifiability would not be worse when more SRPs are provided. Hence, we envisage that the parameter identifiability should be even better given the dataset using the above interleaved sampling scheme.

\section{Appendix A: Fitting performance of models to ex- perimental datasets}

We consider 30 sets of SRPs. Each dataset contains about 200 stimulus-response pairs. Applying the multiple-starting value optimization, we obtain optimal fit of model for each dataset. In Fig. 4.8 and 4.9, we show optimal fits of the HM and the logistic regression model to these datasets. 


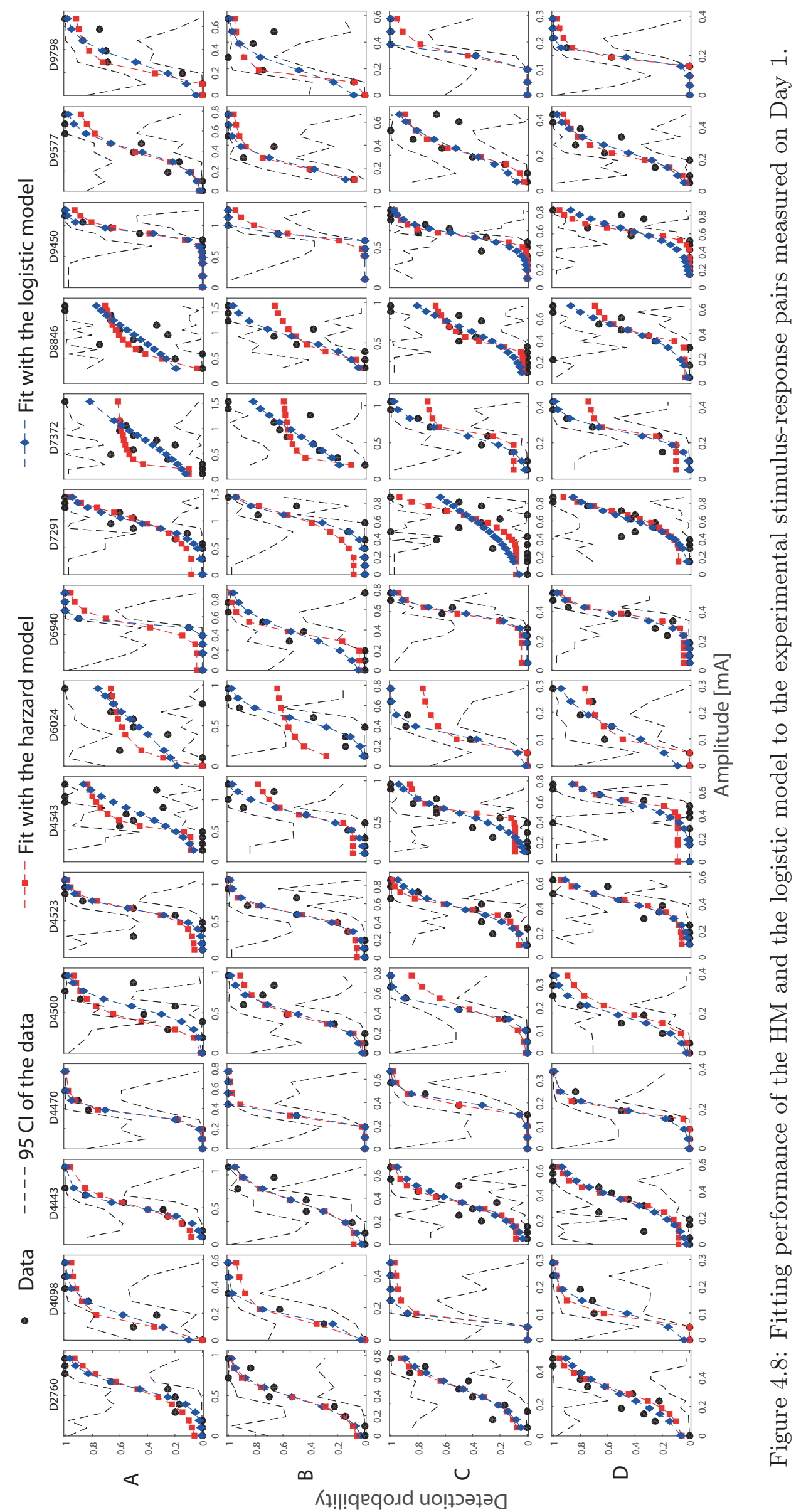



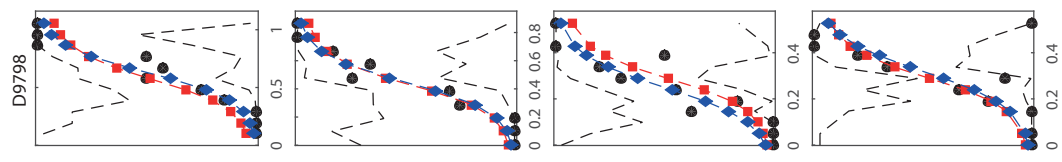

$1 \ldots$
$\ldots$
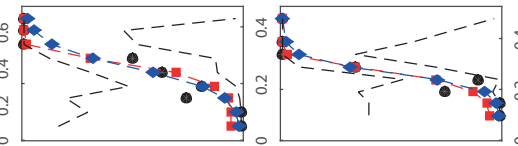

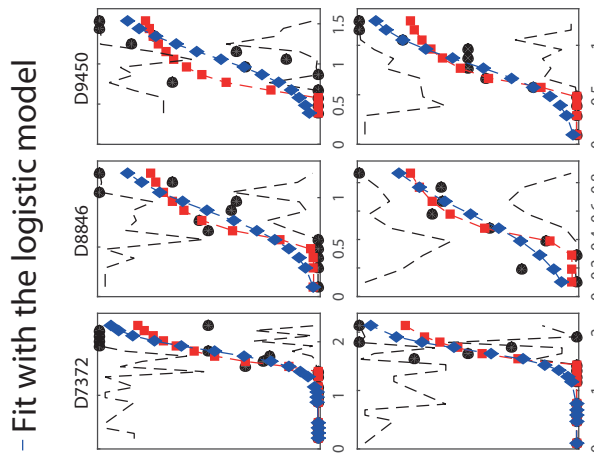

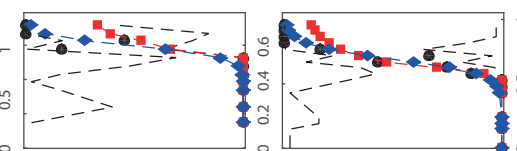

1 .
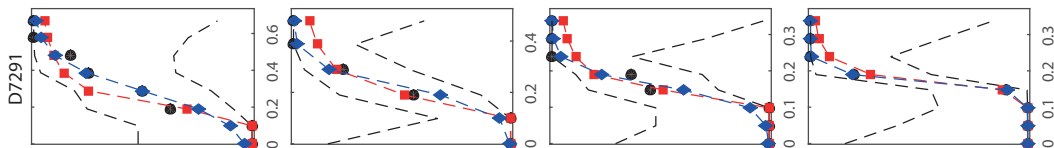

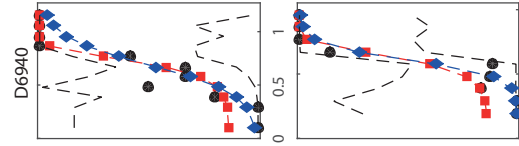
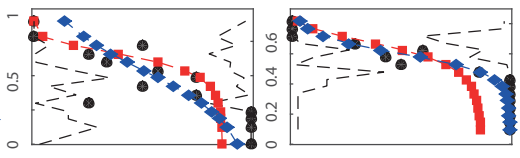

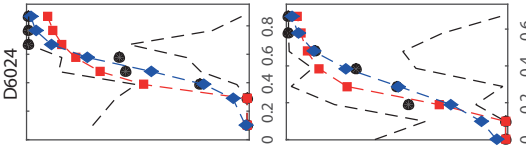
1
1

(......

站

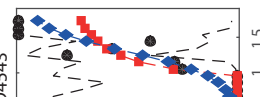

1
120
$1-\equiv=-1$
1
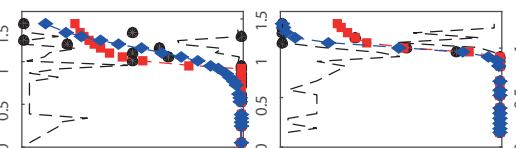

等
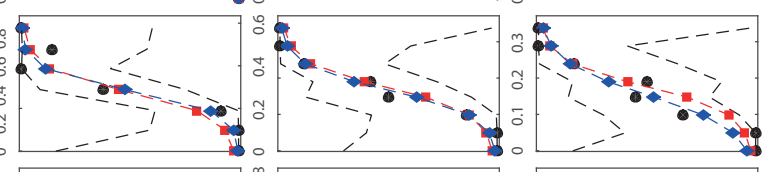

告
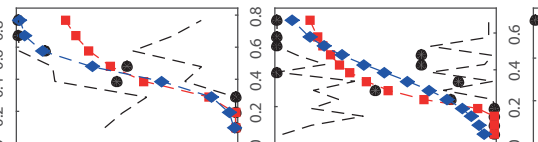

จ่
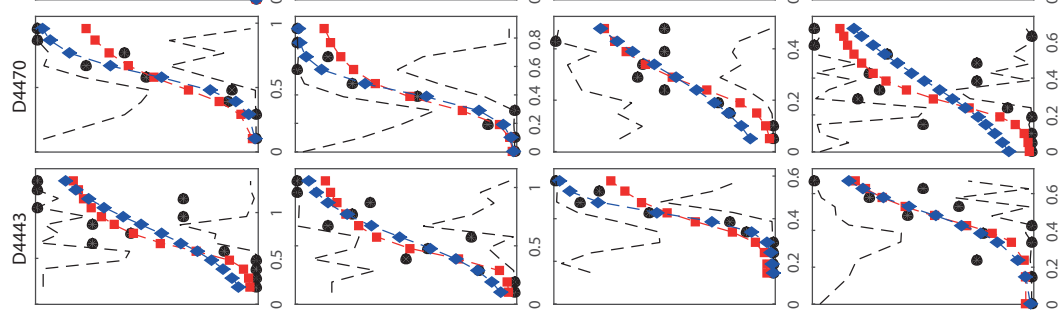

๑ั
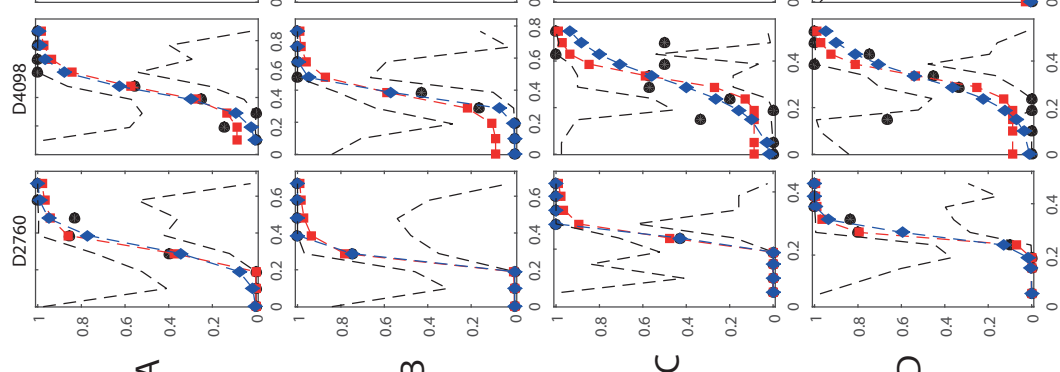

○

Кһ!!!qeqoגd uo!ฺวәғә0 


\section{Appendix B: Optimal model fits and parameter es- timates}

Here we summarize the estimates of six parameters for all 30 elementary datasets. Table 4.3 and 4.4 list parameter estimates for data measured on Day 1 and Day 2 from the multiple-starting-value optimization. In addition, for data from Day 1, we compare the estimates and optimal fits from the PL approach to those using the multiple-starting-value optimization. Comparison shows that there are three cases where even better model fits are found. However, the difference among the three paired comparisons of $-2 \log (L)$ is always smaller than 0.04 . Hence, in the optimization with more extension searching of the parameter space, no significantly better model fits are found. This suggests that the multiple-starting-value optimization with $N_{s}=100$ is sufficient.

Table 4.3: Parameter estimates and optimal fits for 15 elementary datasets using $T S_{1}$ measured on Day 1 using the multiple-starting-value method. We perform profile likelihood method, detecting three cases with different estimates and fits. See those differences in the red.

\begin{tabular}{|c|c|c|c|c|c|c|c|}
\hline Model parameter & $\alpha_{1}[\mathrm{~mA}]$ & $\tau_{1}[\mathrm{~ms}]$ & $\tau_{2}[\mathrm{~ms}]$ & $\alpha_{L}[\mathrm{~A} / \mathrm{s}]$ & $\sigma_{L}[\mathrm{~A} / \mathrm{s}]$ & $\lambda_{L}[\mathrm{kHz}]$ & $-2 \log (\hat{L})$ \\
\hline D2760 & 0.000001 & 0.75 & 649.9 & 0.0014 & 0.000241 & 0.0096 & 201.33 \\
\hline \multirow[t]{2}{*}{$\mathrm{D} 4098$} & 0.000573 & 0.04 & 9.8 & 0.0194 & 0.000000 & 0.1215 & 103.86 \\
\hline & 0.000004 & 0.01 & 17.6 & 0.0124 & 0.000000 & 0.0802 & 103.83 \\
\hline D4443 & 0.083333 & 0.51 & 1000.0 & 0.0008 & 0.000163 & 0.0042 & 219.13 \\
\hline D4470 & 0.033727 & 0.26 & 1000.0 & 0.0007 & 0.000058 & 0.0052 & 98.41 \\
\hline D4500 & 0.000001 & 0.20 & 1000.0 & 0.0009 & 0.000146 & 0.0025 & 174.21 \\
\hline D4523 & 0.095231 & 0.40 & 166.0 & 0.0068 & 0.000935 & 0.0438 & 162.85 \\
\hline D4543 & 0.368583 & 0.11 & 16.6 & 0.0200 & 0.002899 & 0.0470 & 192.49 \\
\hline \multirow[t]{2}{*}{$\overline{\mathrm{D}} \overline{6} 02 \overline{4}$} & 0.001621 & 0.02 & 4.3 & $0.026 \overline{5}$ & $0.00000 \overline{1}$ & 0.0694 & 185.89 \\
\hline & 0.001590 & 0.02 & 4.3 & 0.0265 & 0.000000 & 0.0694 & 185.86 \\
\hline $\mathrm{D} 6940$ & 0.161138 & $0 . \overline{4}$ & 1000.0 & 0.0005 & $\overline{0.000096}$ & 0.0034 & 166.32 \\
\hline D7291 & 0.115707 & 0.93 & 1000.0 & 0.0032 & 0.000219 & 99.9803 & 202.85 \\
\hline D7372 & 0.230783 & 0.01 & 2.0 & 0.0050 & 0.000713 & 0.0586 & 223.15 \\
\hline D8846 & 0.194096 & 0.28 & 67.0 & 0.0047 & 0.000771 & 0.0076 & 227.76 \\
\hline D9450 & 0.093299 & 0.44 & 72.9 & 0.0174 & 0.001357 & 0.0677 & 161.31 \\
\hline D9577 & 0.006398 & 3.00 & 1000.0 & 0.0001 & 0.000021 & 0.0021 & 176.13 \\
\hline \multirow[t]{2}{*}{$\overline{\mathrm{D}} \overline{9} 79 \overline{8}$} & 0.006116 & 1.38 & 9.2 & $0.009 \overline{7}$ & 0.000368 & 0.1427 & 119.37 \\
\hline & 0.006109 & 1.38 & 9.2 & 0.0097 & 0.000368 & 0.1428 & 119.37 \\
\hline
\end{tabular}


Table 4.4: Parameter estimates and optimal fits for 15 elementary datasets using $T S_{1}$ measured on Day 2 using the multiple-starting-value method.

\begin{tabular}{cccccccc}
\hline Model parameter & $\alpha_{1}[\mathrm{~mA}]$ & $\tau_{1}[\mathrm{~ms}]$ & $\tau_{2}[\mathrm{~ms}]$ & $\alpha_{L}[\mathrm{~A} / \mathrm{s}]$ & $\sigma_{L}[\mathrm{~A} / \mathrm{s}]$ & $\lambda_{L}[\mathrm{kHz}]$ & $-2 \log (\hat{L})$ \\
\hline D2760 & 0.132516 & 0.27 & 38.2 & 0.0062 & 0.000533 & 0.0609 & 76.34 \\
D4098 & 0.143050 & 0.30 & 285.5 & 0.0023 & 0.000396 & 0.0157 & 161.70 \\
D4443 & 0.065499 & 0.55 & 63.0 & 0.0104 & 0.001392 & 0.0238 & 190.21 \\
D4470 & 0.000001 & 0.65 & 1000.0 & 0.0006 & 0.000106 & 0.0018 & 224.35 \\
D4500 & 0.000001 & 0.42 & 1000.0 & 0.0007 & 0.000031 & 0.0014 & 188.11 \\
D4523 & 0.000001 & 0.31 & 1000.0 & 0.0009 & 0.000138 & 0.0061 & 149.26 \\
D4543 & 0.603967 & 0.35 & 84.0 & 0.0027 & 0.000000 & 0.0070 & 184.21 \\
D6024 & 0.000001 & 1.98 & 1000.0 & 0.0002 & 0.000000 & 0.0023 & 153.29 \\
D6940 & 0.000001 & 0.21 & 8.2 & 0.2905 & 0.020524 & 100.000 & 192.16 \\
D7291 & 0.062048 & 0.20 & 51.4 & 0.0050 & 0.000085 & 0.0312 & 125.72 \\
D7372 & 0.052303 & 0.13 & 259.7 & 0.0163 & 0.000000 & 0.0143 & 156.32 \\
D8846 & 0.206076 & 0.50 & 50.3 & 0.0064 & 0.000862 & 0.0173 & 196.52 \\
D9450 & 0.355383 & 0.21 & 45.0 & 0.0118 & 0.000000 & 0.0195 & 188.95 \\
D9577 & 0.105230 & 0.25 & 1000.0 & 0.0023 & 0.000151 & 100.000 & 131.10 \\
D9798 & 0.000001 & 0.39 & 1000.0 & 0.0012 & 0.000212 & 0.0039 & 209.46 \\
\hline
\end{tabular}

\section{Appendix C: Computation of profile likelihood}

Here we give the details on the computation of profile likelihood. Given the estimate $\boldsymbol{\theta}$, we compute the profile likelihood according to (4.13). For each single parameter $\boldsymbol{\theta}_{i}$, we divide the computation for the entire parameter range into two sub-interval by the estimate $\boldsymbol{\theta}_{i}$. For each sub-interval, we use an adaptive method to determine step $\Delta_{\theta}$. For illustrative purposes, we explore values of the parameter $\boldsymbol{\theta}_{i}^{(n)}$ in the increasing direction with respect to $\hat{\boldsymbol{\theta}}_{i}$,

$$
\boldsymbol{\theta}_{i}^{(n)}=\boldsymbol{\theta}_{i}^{(n-1)}+\Delta_{\theta}, \quad n=1,2, \ldots, 500,
$$

where $\boldsymbol{\theta}_{i}^{(0)}:=\hat{\boldsymbol{\theta}}_{i}$. The step $\Delta_{\theta}$ could be solved from the univariate equation about $\Delta_{\theta}:-2 \log \left(L\left(\boldsymbol{\theta}_{i}^{(n)}\right)\right)=-2 \log \left(L\left(\boldsymbol{\theta}_{i}^{(n-1)}\right)\right)+q \chi_{\alpha}^{2}$, where by default we set $q=0.05$. If this equation is not solvable, we set $\boldsymbol{\theta}_{i}^{(n)}=1.05 \boldsymbol{\theta}_{i}^{(n-1)}$. Given the value of $\boldsymbol{\theta}_{i}^{(n)}$, the profile likelihood in (4.13) is determined by re-optimizing values of remaining parameters $\boldsymbol{\theta}_{\{/ i\}}$. 


\section{Appendix D: Profile likelihood results with interior estimates for experiments using $T S_{1}$}

Within the 15 elementary datasets on Day 1 using $T S_{1}$, there are 5 sets of SRPs yielding in interior estimates of $\boldsymbol{\theta}$ : for subjects D4523, D4543, D8846, D9450, D9798. For two combined datasets for subjects D2760 and D4443, the profile likelihood was also performed to investigated identifiability of parameters. We have already showed the profile likelihood for D9798 and D4443 in Fig. 4.4 and 4.5, respectively. For other five cases, we show the profile likelihood in Fig. 4.10-4.14.

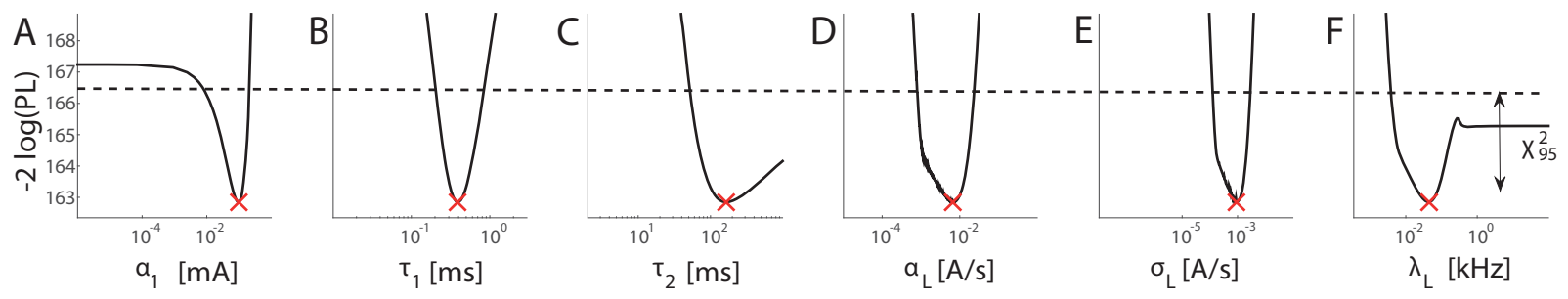

Figure 4.10: PL results for the elementary dataset from subject D4523 measured on Day 1.

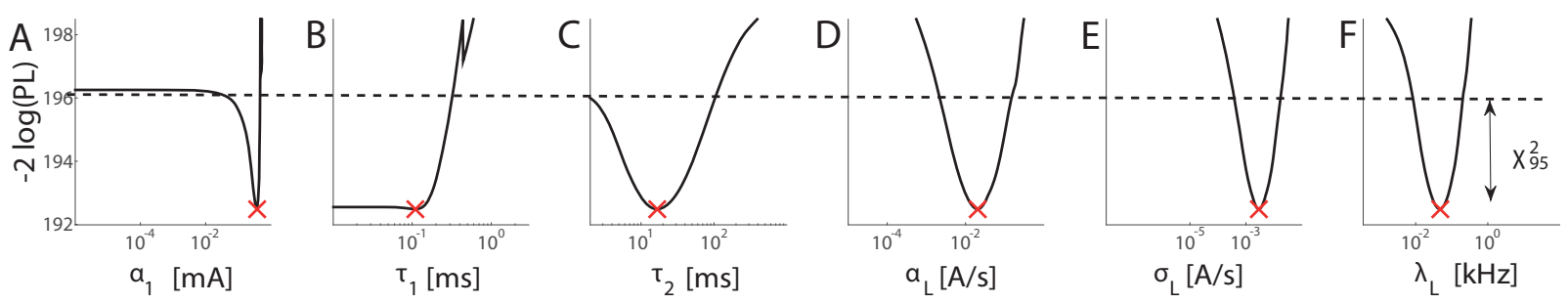

Figure 4.11: PL results for the elementary dataset from subject D4543 measured on Day 1.

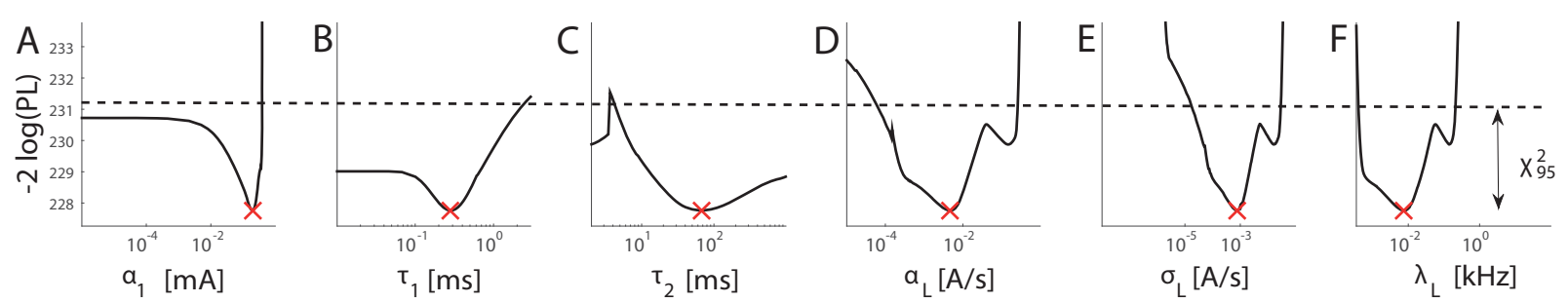

Figure 4.12: PL results for the elementary dataset from subject D8846 measured on Day 1. 


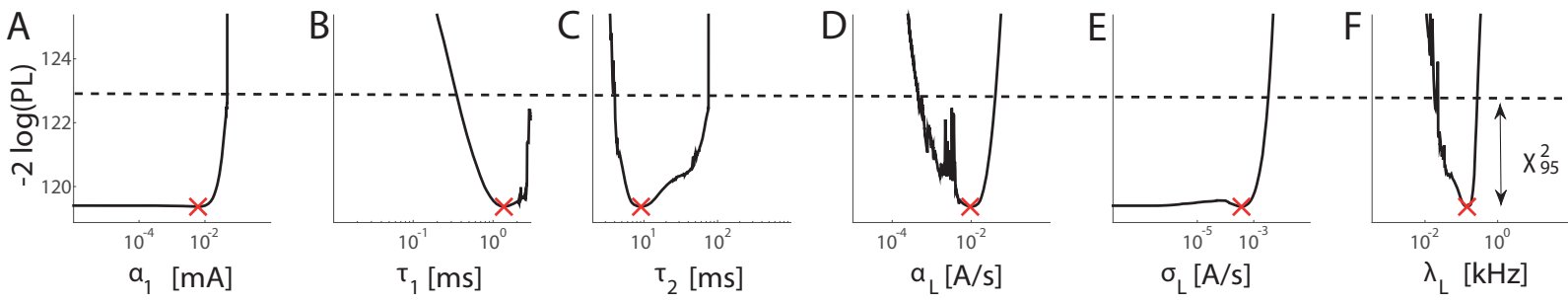

Figure 4.13: PL results for the elementary dataset from subject D9798 measured on Day 1.

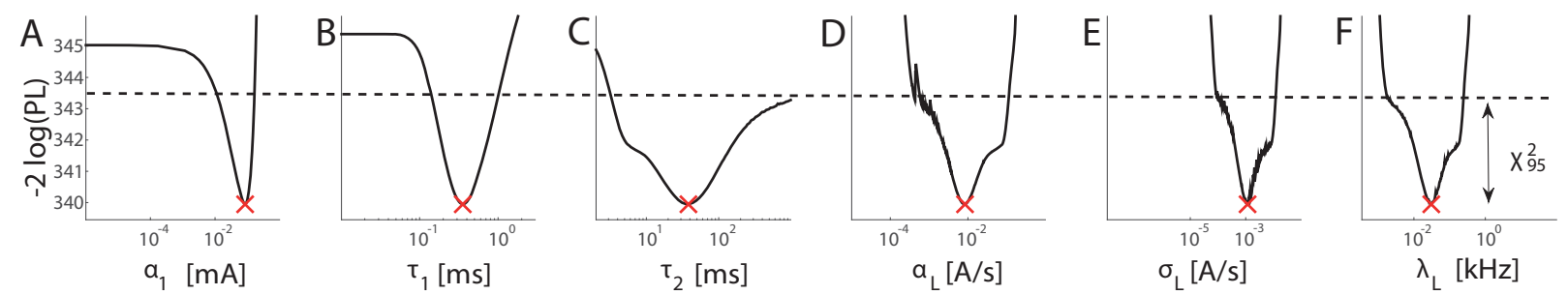

Figure 4.14: PL results for the combined dataset from subject D2760 measured on Day 1 and 2.

\section{Appendix E: The quantity $r$ is a decreasing function of $P W$}

We have

$$
r=\frac{1-\exp \left(-\frac{P W}{\tau_{1}^{l}}\right)}{1-\exp \left(-\frac{P W}{\tau_{1}^{u}}\right)}
$$

The derivative of $r$ with respect to $P W$ is given by

$$
\frac{d r}{d P W}=\frac{\exp \left(P W\left(\frac{1}{\tau_{1}^{u}}-\frac{1}{\tau_{1}^{l}}\right)\right)\left(\tau_{1}^{l}\left(1-\exp \left(P W / \tau_{1}^{l}\right)\right)+\tau_{1}^{u}\left(\exp \left(P W / \tau_{1}^{u}\right)-1\right)\right)}{\tau_{1}^{l} \tau_{1}^{u}\left(\exp \left(P W / \tau_{1}^{u}\right)-1\right)^{2}}
$$

Given $\tau_{1}^{l}<\tau_{1}^{u}$, one has that the sign of $\frac{d r}{d P W}$ is identical to

$$
r_{1}:=\tau_{1}^{l}\left(1-\exp \left(P W / \tau_{1}^{l}\right)\right)+\tau_{1}^{u}\left(-1+\exp \left(P W / \tau_{1}^{u}\right)\right)
$$

The derivative of $r_{1}$ with respect to $P W$ is $\frac{d r_{1}}{d P W}=\exp \left(P W / \tau_{1}^{u}\right)-\exp \left(P W / \tau_{1}^{l}\right)<0$, indicating that $r_{1}$ decreases as $P W$ increases. For $P W=0$, we have $r_{1}=0$, so $r_{1}$ is negative for positive $P W$, and $\frac{d r_{1}}{d P W}<0$ as well. So the quantity $r$ is a deceasing function of $P W$ according to (4.24). 


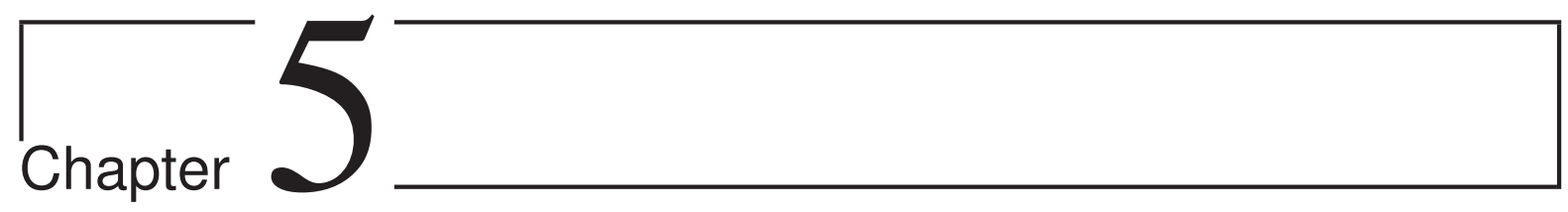

Threshold estimates from logistic regression on stimulus-response pairs from staircase procedures with a case study of a nociceptive detection task 


\section{Abstract}

Threshold measurements are frequently used in psychophysics to assess underlying sensory function. The non-parametric simple staircase procedure adaptively samples stimulus amplitudes near the detection threshold. Recently in a nociceptive detection task, an adaptive probing procedure was proposed by introducing a moving set of amplitudes into the simple staircase procedure. In parametric approaches to estimate the detection threshold, a generalized linear function, e.g. a logistic curve, is often used. A previous simulation study reported a bias in the threshold, i.e. the difference between the expectation of estimates and the actual one, due to a parametric mismatch between underlying psychometric and estimation curves. A thorough analysis on the bias has not been presented for both simple staircase and adaptive probing procedures. Here, we assess the bias in a more mathematically rigorous way. We formulate the staircase procedures as Markov models. We investigate these models regarding stationary and asymptotic sampling distributions of applied amplitudes. The irreducibility of these models guarantees the equivalence of those two distributions. We generate data by using a neurophysiologically plausible model of nociceptive processing. Performing logistic regression on simulated data, we investigate the performance on estimated thresholds in both asymptotic and various finite-sample cases. Simulation results reveal multiple peaks in asymptotic sampling distributions in adaptive probing procedures. In contrast, the simple staircase procedure samples unimodally and more densely around the detection threshold. With the parametric mismatch between logistic and model-based psychometric curves, our simulation results show a larger bias in estimated thresholds for an adaptive probing procedure, compared to the simple procedure. In case of stationary psychometric curves, our study suggests to use the simple staircase procedure for a better estimation of the detection threshold. 


\section{$5.1 \quad$ Introduction}

A psychometric curve describes the overall relation between the stimulus intensity and the subjective response for an individual subject. For a yes-or-no detection task, one important characteristic of the curve is the detection threshold, defined as the amplitude with 0.5 probability to detect the stimulus (Treutwein, 1995; Doll et al., 2014). Thresholds from quantitative sensory testing (QST) are clinically relevant to assess the nociceptive system, which could lead to improved pain management (Wilder-Smith et al., 2003).

If the estimates of individual detection thresholds have a diagnostic purpose, one desires the bias in the estimate to be small. This depends on two factors, the stimulus selection procedure and the estimation method. The aim of the stimulus selection procedure is to apply stimulus amplitudes mostly around the targeted probability as this improves the estimate. However, the detection threshold is not known a priori and hence an adaptive procedure is needed to explore a range of amplitudes. Typically, adaptive procedures are non-parametric staircase methods where the applied amplitude (or dose intensity) for the next trial is increased if the stimulus is not detected, and decreased otherwise. Simple stepping rules for the target probability 0.5 have been proposed decades ago, see e.g. (Dixon and Mood, 1948; Wetherill, 1963). They have been adapted for other target probabilities, e.g. the k-in-a-row staircase (Kingdom and Prins, 2010). As the number of trials approaches infinity, such procedures produce a random walk with a stationary distribution of applied amplitudes (Derman, 1957; Oron and Hoff, 2009). The mode of this distribution, i.e. the stimulus amplitude most often applied, has been shown to be close to the target probability (Oron and Hoff, 2009). Hence, for an experiment, one would choose an adaptive procedure where the mode of the distribution of sampled amplitudes is nearest to the detection threshold as this leads to more stimuli close to the threshold and a better estimate.

The estimation method to determine the threshold from the stimulus-response pairs also affects the estimation performance. A simulation study (García-Pérez and Alcalá-Quintana, 2005) considered various staircase procedures and found that maximum likelihood approach performed better than least square methods to determine threshold estimates. The same study also pointed out that the bias in the estimate was not negligible due to a parametric mismatch between two psychometric functions. The first one is used to simulate the data, and the second one is for a 
parametric estimation purpose. The precise effect of the mismatch on the bias of thresholds, however, was not covered in their simulation study.

Here, we consider a procedure proposed in (Doll et al., 2014) for a nociceptive detection task using electrocutaneous stimulation, which we refer to as the adaptive probing procedure (APP). It collects stimulus-response pairs by introducing an additional stochastic factor in selecting the stimulus amplitude to avoid subjects' anticipation. Instead of a single amplitude, APP randomly chooses the amplitude from a set with $N_{A}$ amplitudes. Then, depending on the response, all amplitudes are increased or decreased. If the set consists of one element, i.e. $N_{A}=1$, we recover the conventional simple staircase procedure (SSP). Examples with sampling trajectories for both procedures are shown in Fig. 5.1. Doll et al. (2014) performed a simulation study to estimate thresholds using logistic regression and determine the bias in SSP and APP. The reported bias was small and decreased if the number of trials was increased, in line with results from (García-Pérez and Alcalá-Quintana, 2005). In the work by Doll et al. (2014), the psychometric functions used for the
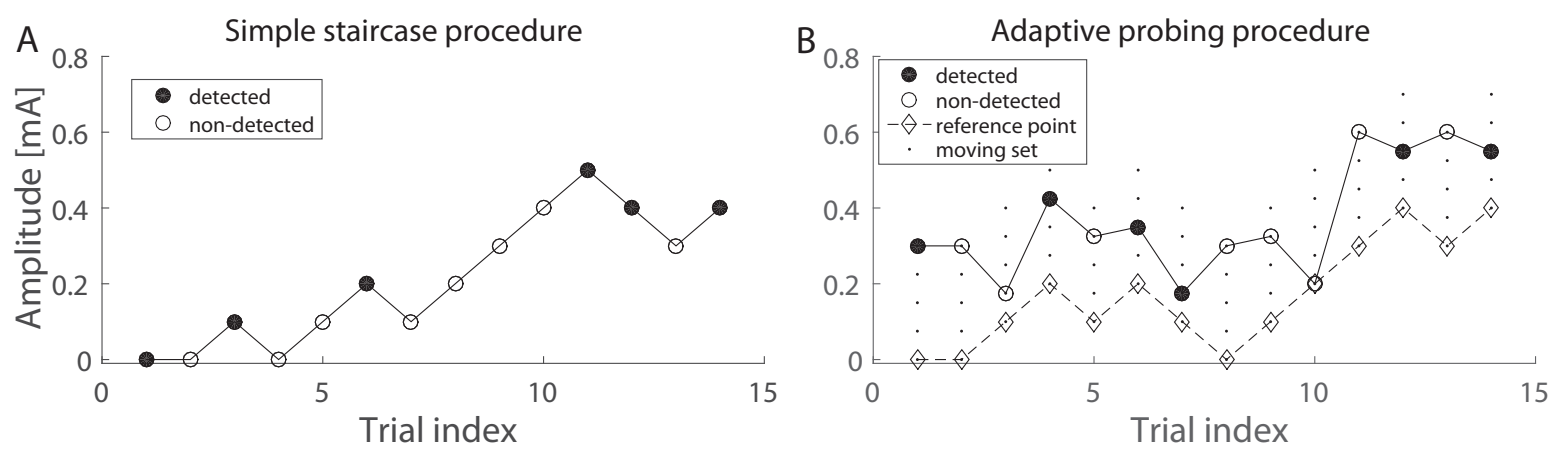

Figure 5.1: Illustrative examples with stimulus-response pairs for two adaptive procedures used in the nociceptive detection task. A: applied amplitudes selected with the simple staircase procedure. B: applied amplitudes selected with the adaptive probing procedure. All possible amplitudes from moving sets are also shown for each trial. The lowest amplitude corresponds to the reference point.

simulation and estimation purposes had the same parametric form. As mentioned above, a mismatch between these two psychometric functions might impede the estimation by introducing a bias in thresholds (García-Pérez and Alcalá-Quintana, 2005). Recently, we developed a neurophysiologically plausible computational model representing peripheral and central nociceptive subsystems in the A $\delta$-fiber-mediated pathway (Yang et al., 2015a). This model provided a reasonable fit to experimental stimulus-response pairs (Yang et al., 2015c). Crucially for this work, the fitted 
psychometric function based on the computational model could differ substantially from a logistic curve. It is not known how APP with logistic regression performs if this mismatch is present.

In this paper, we analyze APP in view of its threshold estimated from logistic regression. We first describe the two adaptive staircase procedures used in the nociceptive detection task. Next, we formulate both staircases procedures as Markov models and study properties of the stationary distributions. We investigate whether and how the different procedures affect distributions of applied amplitudes. Second, we perform Monte Carlo simulations generating data using APP and the neurophysiologically plausible model. Thresholds are estimated by logistic regression with different amounts of stimulus-response pairs. By varying the number of amplitudes $N_{A}$, we assess whether APP is better than SSP with respect to the bias. Based on both theoretical understanding and simulation results, we give some practical recommendation in the choice of these two procedures.

\subsection{Staircase procedures in a nociceptive detection task}

In a nociceptive detection task (Doll et al., 2014), an intra-epidermal needle electrode delivered a current pulse train with a square waveform. The waveform was characterized by three temporal properties, i.e. the number of the pulses $\left(N_{o P}\right)$, the inter-pulse interval $(I P I)$, the pulse width $(P W)$ and also the amplitude $A$. Applying a single stimulus, one binary response $R$ was obtained. If the subject detected the stimulus, we had $R=1$; otherwise $R=0$. Fixing the three temporal properties, two adaptive procedures to select the amplitude, i.e. SSP and APP, were implemented. We describe these procedures in more detail.

For the simple staircase procedure, suppose the response to the current stimulus with amplitude $A_{k}$ at trial number $k$ is 'detected', i.e. $R_{k}=1$. Then, in the next trial, the amplitude for the next trial will decrease by a fixed step size $\Delta$, i.e. $A_{k+1}:=A_{k}-\Delta$. Similarly, if $R_{k}=0$, the amplitude at the next trial will increase by the same step size $\Delta$. The range of applied amplitudes is limited. Negative amplitudes are irrelevant in a detection task, while an upper limit is required for both safety and preferential activation of A $\delta$ fibers (Doll et al., 2014). Therefore, the stepping rule at the boundary is different as the amplitude cannot exceed it. 
Namely, the amplitude stays at the lower boundary or the upper boundary, in case of a detected stimulus or an unnoticed stimulus, respectively. We denote the lower and upper boundaries of the applied amplitude $A$ as $A^{l}$ and $A^{u}$, respectively. In an experiment, the stepping rule results in a staircase pattern for the sequence of applied amplitudes, as illustrated in Fig. 5.1.

The stepping rule for the adaptive probing procedure has two stochastic components. In addition to the subject's variability from trial to trial, the applied amplitude is randomly selected from a moving set $\mathbb{M}$ with equal probability. The set for the $k$ th trial is given by $\mathbb{M}_{k}:=B_{k}+\boldsymbol{\Lambda}$ with $\boldsymbol{\Lambda}:=\left\{0, \delta_{A}, \ldots,\left(N_{A}-1\right) \delta_{A}\right\}$ containing $N_{A}$ possible amplitudes with $\delta_{A}>0$ the intra-set difference. If $R_{k}=1$, then every element for the next trial is decreased with step size $\Delta_{B}$, and increased with the same step size if $R_{k}=0$. If we consider the smallest element $B_{k}$ of $\mathbb{M}_{k}$ as a reference point, then the stepping rule for the reference point including boundary conditions is given by

$$
B_{k+1}=\left\{\begin{array}{cll}
B_{k}+\Delta_{B}\left(1-2 R_{k}\right) & , & \text { if } B^{l}<B_{k}<B^{u}, \\
B_{k} & , & \text { if }\left(B_{k}=B^{l} \text { and } R_{k}=1\right) \text { or }\left(B_{k}=B^{u} \text { and } R_{k}=0\right),
\end{array}\right.
$$

where $B^{l}$ and $B^{u}$ are the lower and upper boundaries of $B$. For the reference point, we set $B^{l}=A^{l}=0$. For the upper one, we know $B^{u}<A^{u}-\left(N_{A}-1\right) \delta_{A}$ as $A_{k} \in \mathbb{M}_{k}$. Therefore, we set

$$
B^{u}:=\left\lfloor\frac{A^{u}-\left(N_{A}-1\right) \delta_{A}}{\Delta_{B}}\right\rfloor \Delta_{B}
$$

The actually applied amplitude is chosen with equal probability from the set $\mathbb{M}_{k}$

$$
\operatorname{Pr}\left(A_{k} \mid B_{k}\right)=\left\{\begin{aligned}
\frac{1}{N_{A}} \quad, & \text { if } A_{k} \in \mathbb{M}_{k} \\
0 & , \quad \text { otherwise. }
\end{aligned}\right.
$$

Fig. 5.1B illustrates APP for $N_{A}=5$ and $\delta_{A}=0.075 \mathrm{~mA}$ as used in (Doll et al., 2014). Note that, if we set $N_{A}=1$, we recover SSP from APP.

\subsection{Markov models for staircase procedures and their properties}

In this section, we formulate the APP as a Markov model. This is in contrast to the SSP which is a Markov chain. We study properties of these Markov models. In particular, we focus on qualitative understanding of the shape of asymptotic sampling distributions of both reference points and applied amplitudes. 


\subsubsection{Markovian formulation}

We first introduce two sets $\mathbb{B}$ and $\mathbb{A}$ containing all feasible reference points or applied amplitudes, respectively. Then, we sort elements in $\mathbb{B}$ in ascending order as $\left\{b_{j}\right\}$, with $j=1,2, \ldots, n(\mathbb{B})$. Here, $n(\mathbb{U})$ denotes the number of elements of $\mathbb{U}$. Considering moving sets, we have $\mathbb{A}:=\cup_{j=1}^{n(\mathbb{B})}\left(b_{j}+\boldsymbol{\Lambda}\right)$ containing $\left\{a_{i}\right\}$ with $i=1,2, \ldots, n(\mathbb{A})$ in ascending order for set $\mathbb{A}$.

Now, we formulate the stepping and assigning rule for those two kinds of stochasticity in the APP. The former rule is merely applicable to $\mathbb{B}$. While, the later rule in (5.3) governs how to assign amplitudes in $\mathbb{A}$ from reference points in $\mathbb{B}$. For these two rules, we use two matrices $\mathbf{A} \in \mathbb{R}^{n(\mathbb{A}) \times n(\mathbb{B})}$ and $\mathbf{B} \in \mathbb{R}^{n(\mathbb{B}) \times n(\mathbb{B})}$, respectively. For the assigning rule, we have the matrix element

$$
\boldsymbol{A}_{i, j}:=\operatorname{Pr}\left(A=a_{i} \mid B=b_{j}\right)
$$

which is independent of $k$. For the stepping rule, the matrix element $\boldsymbol{B}_{i, j}:=$ $\operatorname{Pr}\left(B_{k+1}=b_{i} \mid B_{k}=b_{j}\right)$ for any two consecutive trials $k$ and $k+1$. As two consecutive trials only explore adjacent reference points, matrix $\boldsymbol{B}$ is a sparse matrix. Here, we specify its non-zero elements. According to Bayesian theorem, for $1<j<n(\mathbb{B})$, we have $\boldsymbol{B}_{j-1, j}$ as

$$
\begin{aligned}
\operatorname{Pr}\left(B_{k+1}=b_{j-1} \mid B_{k}=b_{j}\right) & =\operatorname{Pr}\left(R_{k}=1 \mid B_{k}=b_{j}\right)=\frac{\operatorname{Pr}\left(R_{k}=1, B_{k}=b_{j}\right)}{\operatorname{Pr}\left(B_{k}=b_{j}\right)} \\
& =\frac{\sum_{l} \operatorname{Pr}\left(R_{k}=1 \mid A_{k}=a_{l}, B_{k}=b_{j}\right) \operatorname{Pr}\left(A_{k}=a_{l} \mid B_{k}=b_{j}\right) \operatorname{Pr}\left(B_{k}=b_{j}\right)}{\operatorname{Pr}\left(B_{k}=b_{j}\right)} \\
& =\sum_{l} \operatorname{Pr}\left(R_{k}=1 \mid A_{k}=a_{l}\right) \operatorname{Pr}\left(A_{k}=a_{l} \mid B_{k}=b_{j}\right) \\
& =\sum_{l} \boldsymbol{A}_{l, j} \operatorname{Pr}\left(R_{k}=1 \mid A_{k}=a_{l}\right) .
\end{aligned}
$$

Analogously, we obtain $\boldsymbol{B}_{j+1, j}=\sum_{l=1}^{n(\mathbb{A})} \boldsymbol{A}_{l, i}\left(1-\operatorname{Pr}\left(R_{k}=1 \mid A_{k}=a_{l}\right)\right)$. In case of $B=b_{1}$ or $B=b_{n(\mathbb{B})}$, we have $\boldsymbol{B}_{1,1}=\sum_{l=1}^{n(\mathbb{A})} \boldsymbol{A}_{l, 1} \operatorname{Pr}\left(R_{k}=1 \mid A_{k}=a_{l}\right)$ and $\boldsymbol{B}_{n(\mathbb{B}), n(\mathbb{B})}=\sum_{l=1}^{n(\mathbb{A})} \boldsymbol{A}_{l, n(\mathbb{B})}\left(1-\operatorname{Pr}\left(R_{k}=1 \mid A_{k}=a_{l}\right)\right)$. The conditional probabilities $\operatorname{Pr}\left(R_{k}=1 \mid A_{k}=a_{l}\right)$ are provided by the stationary psychometric function as

$$
\Psi_{l}:=\operatorname{Pr}\left(R=1 \mid A=a_{l}\right)
$$

Next, defining $\boldsymbol{\Phi}:=\boldsymbol{A}^{\prime} \boldsymbol{\Psi}$ with $\boldsymbol{\Psi}:=\left(\Psi_{1}, \Psi_{2}, \ldots, \Psi_{n(\mathbb{A})}\right)^{\prime}$, we write the element of 
matrix $\mathbf{B}$ as

$$
\boldsymbol{B}_{i, j}=\left\{\begin{aligned}
\boldsymbol{\Phi}_{j} & , \quad \text { if } j>1 \text { and } i=j-1, \\
1-\boldsymbol{\Phi}_{j} & , \quad \text { if } j<n(\mathbb{B}) \text { and } i=j+1, \\
\boldsymbol{\Phi}_{1} & , \quad \text { if } i=j=1, \\
1-\boldsymbol{\Phi}_{n(\mathbb{B})}, & \text { if } i=j=n(\mathbb{B}) \\
0 \quad & \text { otherwise. }
\end{aligned}\right.
$$

In order to give a matrix representation, we introduce two probability distributions $\boldsymbol{P}_{k}^{\mathbb{A}}:=\left(P_{k, 1}^{\mathbb{A}}, P_{k, 2}^{\mathbb{A}}, \ldots, P_{k, n(\mathbb{A})}^{\mathbb{A}}\right)^{\prime}$ and $\boldsymbol{P}_{k}^{\mathbb{B}}:=\left(P_{k, 1}^{\mathbb{B}}, P_{k, 2}^{\mathbb{B}}, \ldots, P_{k, n(\mathbb{B})}^{\mathbb{B}}\right)^{\prime}$ with $P_{k, i}^{\mathbb{A}}:=$ $\operatorname{Pr}\left(A_{k}=a_{i}\right)$ and $P_{k, j}^{\mathbb{B}}:=\operatorname{Pr}\left(B_{k}=b_{j}\right)$ depending on $k$. Then, we formulate the adaptive probing procedure as a finite-state Markov model

$$
\boldsymbol{P}_{k+1}^{\mathbb{B}}=\mathbf{B} \boldsymbol{P}_{k}^{\mathbb{B}}, \quad \boldsymbol{P}_{k}^{\mathbb{A}}=\mathbf{A} \boldsymbol{P}_{k}^{\mathbb{B}}, \text { for } k \geq 1,
$$

where $\mathbf{B}$ is the transition matrix for the stepping rule and $\mathbf{A}$ is referred to as the observation matrix for the assigning rule. We also remark that elements of the matrix $\mathbf{B}$ depend on the matrix $A$, see (5.5).

\subsubsection{Relation between SSP and APP}

Based on Markovian formulation, we stress the relation between SSP and APP. When $N_{A}=1$, one has $\mathbb{A}=\mathbb{B}$, where the moving set is restricted to a single amplitude. Hence, the reference point $B_{k}$ is identical to the applied amplitude $A_{k}$ for any $k \geq 1$. In addition, matrix $\mathbf{A}$ becomes an identity matrix. So, for SSP, we see that the Markov model (5.8) becomes a Markov chain as

$$
\boldsymbol{P}_{k+1}^{\mathbb{A}}=\mathbf{B} \boldsymbol{P}_{k}^{\mathbb{A}}, \quad k \geq 1
$$

Here, we exemplified the formulated models with specific values of procedure parameters. We assume the procedural set-up as: $\delta_{A}=\Delta_{B}=0.5 \mathrm{~mA}, A^{l}=0 \mathrm{~mA}$ and $A^{u}=2 \mathrm{~mA}$. Then, we have the set of applied amplitudes $\mathbb{A}=\left\{a_{1}=0, a_{2}=\right.$ $\left.0.5, a_{3}=1, a_{4}=1.5, a_{5}=2\right\}$. First, for the SSP, i.e. 'restricted APP' when $N_{A}=1$, we show a five state diagram for the Markov chain in Fig. 5.2. Second, for an APP with the moving set of the range $1 \mathrm{~mA}$, i.e. $N_{A}=3$, we have $\mathbb{B}=\left\{b_{1}=0, b_{2}=\right.$ $\left.0.5, b_{3}=1\right\}$. We show the diagram for the Markov model in Fig. 5.3, where blue and yellow nodes represent reference points and applied amplitudes, respectively. For this APP, one could notice that transitions among states $\mathbb{B}$ have probabilities 


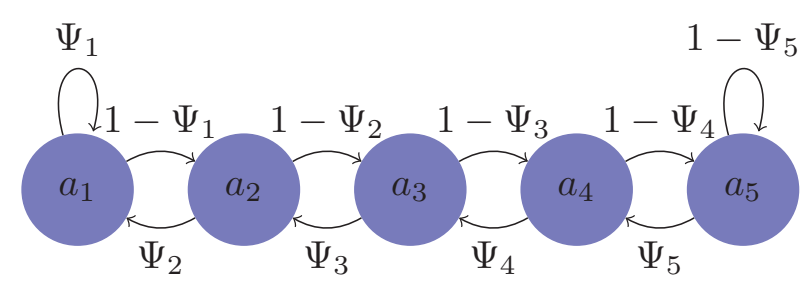

Figure 5.2: A Markov chain with five states in set $\mathbb{A}$. All states can only reach adjacent states except at the boundaries. The transition probability between these adjacent states is either $1-\Psi$ or $\Psi$.

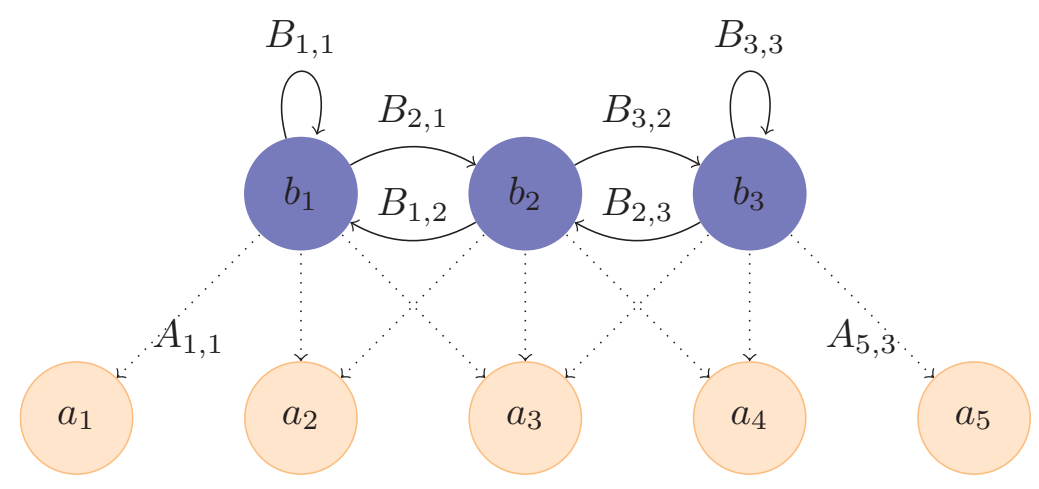

Figure 5.3: A Markov model with three states $\left\{b_{1}, b_{2}, b_{3}\right\}$ and five states $\left\{a_{1}, a_{2}, a_{3}, a_{4}, a_{5}\right\}$ for the reference point and the applied amplitude, respectively. The blue and yellow nodes represent the reference point and the applied amplitude, respectively. The probability $\boldsymbol{B}_{i, j}$ represents the transition probabilities for reference points. The probability $\boldsymbol{A}_{i, j}$ represents the assigning probability from reference points to applied amplitudes. Note that not all $\boldsymbol{A}_{i, j}$ are shown.

$$
\boldsymbol{B}=\left(\begin{array}{ccc}
\boldsymbol{\Phi}_{1} & \boldsymbol{\Phi}_{2} & 0 \\
1-\boldsymbol{\Phi}_{1} & 0 & \boldsymbol{\Phi}_{3} \\
0 & 1-\boldsymbol{\Phi}_{2} & 1-\boldsymbol{\Phi}_{3}
\end{array}\right)
$$

where $\boldsymbol{\Phi}=\boldsymbol{A}^{\prime} \boldsymbol{\Psi}$ with $\boldsymbol{\Psi}=\left(\Psi_{1}, \Psi_{2}, \Psi_{3}, \Psi_{4}, \Psi_{5}\right)^{\prime}$ and

$$
\boldsymbol{A}=\left(\begin{array}{ccc}
\frac{1}{3} & 0 & 0 \\
\frac{1}{3} & \frac{1}{3} & 0 \\
\frac{1}{3} & \frac{1}{3} & \frac{1}{3} \\
0 & \frac{1}{3} & \frac{1}{3} \\
0 & 0 & \frac{1}{3}
\end{array}\right)
$$

Here we remark that, as $n(\mathbb{A}) \neq n(\mathbb{B})$, one cannot formulate transitions among $\mathbb{A}$ as a Markov chain. 


\subsubsection{Asymptotic sampling distributions of reference point and applied amplitude}

To evaluate the estimation performance of detection thresholds, we investigate how the staircase procedures sample applied amplitudes by studying properties of the Markov model. Here, we start with showing that the Markov chain for the reference point is irreducible. Its irreducibility guarantees the existence and uniqueness of the stationary distribution. After that, for both reference point and applied amplitude, we discuss the equivalence of asymptotic sampling distributions to their stationary distributions of the Markov model. We finish with a qualitative understanding of the shape of asymptotic sampling distribution of applied amplitudes.

\subsubsection{Irreducible Markov chain}

Checking whether the Markov chain is irreducible serves as a prerequisite to derive further properties. A conceptual understanding of such irreducibility is that any reference point in one APP can be reached within a finite number of steps from any starting reference point, see the example in Fig. 5.3. The formal definition of an irreducible Markov chain is as follows: for any $q_{1}, q_{2} \in 1,2, \ldots, n(\mathbb{B})$, there exists $n>1$, such that $\operatorname{Pr}\left(B_{n}=b_{q_{2}} \mid B_{1}=b_{q_{1}}\right)>0$.

Before showing that the Markov chain in one APP is irreducible, we first assume that $\Psi_{1}>0$ and $\Psi_{n(\mathbb{A})}<1$. We assume the lowest value of psychometric function $\Psi_{1}>0$, as humans can have a positive probability to detect a sham stimulus. In addition, as low-intensity amplitudes are used in the detection task, one can expect the highest probability $\Psi_{n(\mathbb{A})}<1.1$ Second, we assume that the psychometric function $\Psi$ is a non-decreasing function with respect to the amplitude $A$. Given the assigning rule in (5.3), it follows that from the transition probabilities, we have $\Phi_{1}>0$ or $\Phi_{n(\mathbb{B})}<1$. In addition, the elements $\boldsymbol{\Phi}_{i}$ are non-decreasing with respect to the index $i$ and lie between 0 and 1 .

Now, we are ready to show that the Markov chain of reference points is irreducible. We sketch a proof in the five following mutually exclusive but complementary scenarios:

(a) when $q_{1}=q_{2}=1, \operatorname{Pr}\left(B_{n}=b_{q_{2}} \mid B_{1}=b_{q_{1}}\right) \geq \operatorname{Pr}\left(B_{3}=b_{1} \mid B_{1}=b_{1}\right)=\left(1-\Phi_{1}\right) \Phi_{2}>0$;

\footnotetext{
${ }^{1}$ In a special case where both $\Psi_{1}=0$ and $\Psi_{n(\mathbb{A})}=1$, by restricting applied amplitudes, one can continue to derive the following properties.
} 
(b) when $q_{1}=q_{2}=n(\mathbb{B}), \operatorname{Pr}\left(B_{n}=b_{n(\mathbb{B})} \mid B_{1}=b_{n(\mathbb{B})}\right) \geq \operatorname{Pr}\left(B_{3}=b_{n(\mathbb{B})} \mid B_{1}=b_{n(\mathbb{B})}\right)=$ $\Phi_{n(\mathbb{B})}\left(1-\Phi_{n(\mathbb{B})-1}\right)>0$;

(c) when $\left.1<q_{1}=q_{2}<n(\mathbb{B}), \operatorname{Pr}\left(B_{n}=b_{q_{1}} \mid B_{1}=b_{q_{1}}\right)\right) \geq \operatorname{Pr}\left(B_{3}=b_{q_{1}} \mid B_{1}=b_{q_{1}}\right)=$ $\left(1-\Phi_{q_{1}-1}\right) \Phi_{q_{1}}+\Phi_{q_{1}+1}\left(1-\Phi_{q_{1}}\right)>0$

(d) when $q_{1}<q_{2}, \operatorname{Pr}\left(B_{n}=b_{q_{2}} \mid B_{1}=b_{q_{1}}\right) \geq \operatorname{Pr}\left(B_{q_{2}-q_{1}}=b_{q_{2}} \mid B_{1}=b_{q_{1}}\right)=\Pi_{q=q_{1}}^{q_{2}-1}(1-$ $\left.\Phi_{q}\right)>0$

(e) when $q_{1}>q_{2}, \operatorname{Pr}\left(B_{n}=b_{q_{2}} \mid B_{1}=b_{q_{1}}\right) \geq \operatorname{Pr}\left(B_{q_{1}-q_{2}}=b_{q_{2}} \mid B_{1}=b_{q_{1}}\right)=\Pi_{q=q_{2}}^{q_{1}-1} \Phi_{q}>0$.

Hence, we derive $\operatorname{Pr}\left(B_{n}=b_{q_{2}} \mid B_{1}=b_{q_{1}}\right)>0$, for any $q_{1}, q_{2} \in\{1,2, \ldots, n(\mathbb{B})\}$, indicating an irreducible Markov chain.

\subsubsection{Stationary distributions of the reference point and the applied amplitudes}

One important probability distribution of the Markov chain is its stationary distribution. We denote it as $\boldsymbol{\pi}^{\mathbb{B}}$, satisfying $\boldsymbol{\pi}^{\mathbb{B}}=\mathbf{B} \boldsymbol{\pi}^{\mathbb{B}}$. According to the assigning rule in the APP, one can determine the corresponding stationary distribution of the applied amplitude as

$$
\pi^{\mathbb{A}}=\mathbf{A} \pi^{\mathbb{B}}
$$

Since the Markov chain is finite and irreducible, the stationary distribution will be unique (Durrett, 2012). Hence, given the values of psychometric functions $\boldsymbol{\Psi}$ and matrix $\mathbf{A}$, one can uniquely determine both distributions $\boldsymbol{\pi}^{\mathbb{A}}$ and $\boldsymbol{\pi}^{\mathbb{B}}$.

We continue to study the stationary distribution $\boldsymbol{\pi}^{\mathbb{B}}$. We denote $\boldsymbol{T}:=\boldsymbol{B}-\boldsymbol{I}$, where $\boldsymbol{I}$ is the identity matrix. For a Markov chain, one has $\boldsymbol{T} \boldsymbol{\pi}^{\mathbb{B}}=\mathbf{0}$. By performing row transformations, one finds $\boldsymbol{T}$ the detailed balance

$$
\boldsymbol{\pi}_{i-1}^{\mathbb{B}}\left(1-\Phi_{i-1}\right)=\boldsymbol{\pi}_{i}^{\mathbb{B}} \Phi_{i}, \quad 1<i \leq n(\mathbb{B}) .
$$

In the coming section, we will use (5.13) to study the shape of the stationary distribution of $\pi^{\mathbb{B}}$.

\subsubsection{Equivalence of the asymptotic sampling distribution to the sta- tionary distribution}

We have formulated staircase procedures as Markov models. For such a Markov model, we showed that its stationary distribution is unique. As a probabilistic formulation, the probability distribution in one Markov model describes samples from 
multiple runs of one staircase procedure. In contrast, psychophysical experiments usually collect a single sequence of stimulus-response pairs from one individual. A question then arises, "How our Markov formulation helps to understand properties of staircase procedures in case of a single sequence?" We start to address this by considering the asymptotic situation, i.e. the number of stimulus-response pairs approaches infinity. We denote distributions of sampled reference points or applied amplitudes as asymptotic sampling distributions.

For the irreducible finite-state Markov chain, if follows that there is an equivalence (Durrett, 2012) as

$$
\lim _{N_{S R P} \rightarrow \infty} \frac{1}{N_{S R P}} \sum_{k=1}^{N_{S R P}} \mathbb{1}\left(B_{k}-b_{j}\right)=\pi_{j}^{\mathbb{B}}, \quad \text { for any } j \in\{1,2, \ldots, n(\mathbb{B})\},
$$

where $\mathbb{1}(z)$ is an indicator function, returning 1 if $z=0$, and 0 otherwise. This permits us to study asymptotic sampling distributions by using equivalent stationary distributions for both applied amplitude $A$ and reference point $B$.

Now, we investigate the shape of the distribution $\pi^{\mathbb{A}}$, as the idea behind staircase procedures is to sample amplitudes nearby the detection threshold. For that, we focus on the mode of the distribution of applied amplitudes, which appears as a peak with higher probability than its two adjacent reference points. If a unique mode exists, we say that the distribution is unimodal; otherwise, the distribution is multimodal.

As $\pi^{\mathbb{A}}$ depends $\pi^{\mathbb{B}}$, we first analyze $\pi^{\mathbb{B}}$ by showing that it is unimodal. We assume that psychometric function is strictly increasing near the detection threshold. Following (Oron and Hoff, 2009), we consider two reference points $b_{m}, b_{m+1} \in \mathbb{B}$, satisfying $\Phi_{m} \leq 0.5<\Phi_{m+1}$. One can expect that the unique mode locate at either $b_{m}$ or $b_{m+1}$. (In special cases, it is also possible that both of them are the mode.)

In contract to unimodal $\boldsymbol{\pi}^{\mathbb{B}}$, we remark that there could be multiple peaks in the distribution $\boldsymbol{\pi}^{\mathbb{A}}$. The assigning rule introduces aliasing of the applied amplitude, as the same applied amplitude could be assigned from distinct sets with different reference points. When $\Delta_{B}=\delta_{A}$, a unique mode of $\pi^{\mathbb{B}}$ will be transferred to a unique mode of $\boldsymbol{\pi}^{\mathbb{A}}$. However, in other cases, the distribution could contain multiple local peaks, as aliasing depends on the $N_{A}$. We will illustrate this effect later in the coming section.

So far, we have gained some qualitative insights of the shape of asymptotic sampling distributions $\pi^{\mathbb{B}}$ and $\pi^{\mathbb{A}}$. In the following section, we will focus on more 
quantitative understanding of effects of procedures on estimated thresholds in the nociceptive detection task.

\subsection{Cases studies for the nociceptive detection task}

Given Markovian formulation and properties, we continue with the case for the nociceptive detection task with a newly developed computational model, which represents essential nociceptive mechanisms. To study asymptotic sampling distributions, we illustrate various cases with simulated stimulus-response pairs based on the computational model.

\subsubsection{Model-based psychometric functions}

We denote the model-based psychometric function, i.e. the conditional probability to detect stimulus $S$, as $\Psi(S):=\operatorname{Pr}(R=1 \mid S)$. A single trial may be simulated by drawing a random number $\xi$ from a standard uniform distribution. The response is $R=1$ when $\xi<\Psi(S)$, indicating that the stimulus is detected, and $R=0$ otherwise.

Here we briefly describe the model, for more details see (Yang et al., 2015a). Peripheral activation by the electrical stimulus is described by the threshold-linear function

$$
\left[f_{A}-\alpha_{1}\right]_{+}:=\pi\left(f_{A}-\alpha_{1}\right) H\left(f_{A}-\alpha_{1}\right)
$$

where

$$
f_{A}:=A\left(1-\exp \left(-\frac{P W}{\tau_{1}}\right)\right)
$$

and $H(\cdot)$ is a Heaviside step function; $\tau_{1}$ and $\alpha_{1}$ are the time constant and the activation threshold of afferent fibers, respectively. Next, through synaptic connections, an excitatory post-synaptic current $I_{p}^{*}(t)$ is induced

$$
I_{p}^{*}(t)=\frac{\left[f_{A}-\alpha_{1}\right]_{+}}{\tau_{s}} \sum_{k=0}^{N o P-1} \exp \left(-\frac{t-k I P I}{\tau_{s}}\right) H(t)
$$

with time constant $\tau_{s}=1.5 \mathrm{~ms}$ (Gabbiani et al., 1994). This drives the postsynaptic potential $x(t)$ of a secondary dorsal horn neuron, which we model as a leaky integrator

$$
\tau_{2} \dot{x}=-x+I_{p}^{*}(t), \quad x(0)=0 .
$$


The value of the time constant $\tau_{2}$ is roughly several tens of milliseconds (Prescott and Koninck, 2002; Weng et al., 2006). This noise-free post-synaptic potential is converted into an instantaneous firing rate through a non-homogeneous Poisson process (Plesser and Gerstner, 2000)

$$
\lambda(t)=\lambda_{L}\left(1+\exp \left(\frac{\alpha_{L}-x(t)}{\sigma_{L}}\right)\right)^{-1} .
$$

Here the lumped parameters $\alpha_{L}, \sigma_{L}$ and $\lambda_{L}$ represent the threshold, the slope parameter and the maximal firing rate, respectively. The expected value of the number of spikes during a trial interval of duration $T$ is

$$
\lambda_{T}=\int_{0}^{T} \lambda(t) d t
$$

The binary response $R$ equals one given sufficient activity in the central nociceptive subsystem. In the HM, we assume that sufficient activation implies at least one secondary neuron generated an action potential during the trial interval $T$. So the model-based psychometric function evaluated at the parameters $\boldsymbol{\theta}$ is given by

$$
\Psi_{\boldsymbol{\theta}}=1-\exp \left(-\lambda_{T}\right) .
$$

Our model contains six lumped parameters $\boldsymbol{\theta}=\left(\alpha_{1}, \tau_{1}, \tau_{2}, \alpha_{L}, \sigma_{L}, \lambda_{L}\right)$, which depend on more than ten physical quantities, characterizing peripheral and central nociceptive components. Regarding meaning of lumped parameters, $\alpha_{1}$ and $\tau_{1}$ merely quantify peripheral characteristics, and $\tau_{2}$ and $\lambda_{L}$ merely describe the central properties. But $\alpha_{L}$ and $\sigma_{L}$ are compound characteristics of both subsystems. Regarding the psychometric function values, we remark that, when $A=0$, the psychometric function value is lowest, and also the corresponding $\lim _{\sigma_{L} \rightarrow 0} \Psi_{\boldsymbol{\theta}}=0$.

Based on the HM, we denote the stimulus amplitude $\alpha_{50}^{t}$, satisfying $\Psi_{\boldsymbol{\theta}}(A=$ $\left.\alpha_{50}^{t}\right)=0.5$, as the true detection threshold. We stress that in the HM, the psychometric curve depends on both the model parameters and the temporal properties. To represent plausible psychometric functions, we consider seven vectors of estimates of model parameters from 10-minutes detection experiments (Yang et al., 2015c). We list them in Table 5.1. 
Table 5.1: Maximum likelihood estimates of six model parameters in the hazard model using stimulus-response pairs from 10-minutes detection experiments using TS.

\begin{tabular}{ccccccc}
\hline Model parameter & $\alpha_{1}[\mathrm{~mA}]$ & $\tau_{1}[\mathrm{~ms}]$ & $\tau_{2}[\mathrm{~ms}]$ & $\alpha_{L}[\mathrm{~A} / \mathrm{s}]$ & $\sigma_{L}[\mathrm{~A} / \mathrm{s}]$ & $\lambda_{L}[\mathrm{kHz}]$ \\
\hline $\boldsymbol{\theta}^{(1)}$ & 0.0952 & 0.39 & 166.04 & 0.0068 & 0.000935 & 0.044 \\
$\boldsymbol{\theta}^{(2)}$ & 0.3686 & 0.11 & 16.62 & 0.0200 & 0.002899 & 0.047 \\
$\boldsymbol{\theta}^{(3)}$ & 0.1941 & 0.28 & 66.97 & 0.0049 & 0.000771 & 0.008 \\
$\boldsymbol{\theta}^{(4)}$ & 0.0933 & 0.44 & 72.90 & 0.0174 & 0.001357 & 0.068 \\
$\boldsymbol{\theta}^{(5)}$ & 0.0061 & 1.38 & 9.18 & 0.0097 & 0.000368 & 0.143 \\
$\boldsymbol{\theta}^{(6)}$ & 0.0853 & 0.36 & 37.57 & 0.0082 & 0.001200 & 0.030 \\
$\boldsymbol{\theta}^{(7)}$ & $1.001 \times 10^{-6}$ & 0.46 & 4.83 & 0.0950 & 0.012000 & 0.160 \\
\hline
\end{tabular}

We denote the four experimentally used combinations of temporal stimulus properties by $T S$, see Table 5.2.

Table 5.2: Four combinations of temporal stimulus properties for the electrocutaneous pulse train stimulus. If $N o P=1$, then the $I P I$ is undefined.

\begin{tabular}{ccccc}
\hline Index & $\mathrm{A}$ & $\mathrm{B}$ & $\mathrm{C}$ & $\mathrm{D}$ \\
\hline$N o P[\#]$ & 1 & 1 & 2 & 2 \\
$I P I[\mathrm{~ms}]$ & - & - & 10 & 50 \\
$P W[\mathrm{~ms}]$ & 0.42 & 0.84 & 0.42 & 0.42 \\
\hline
\end{tabular}

In addition to model parameters and temporal parameters, the parameters in staircase procedure also affect the properties of Markov models. Here, we give a summary of their set-up for our following simulations. First, we set the lower and upper boundaries of applied amplitudes as $A^{l}=0$ and $A^{u}=2 \mathrm{~mA}$, respectively. Second, for APP, we set the intra-set difference in $\boldsymbol{\Lambda}$ according to its dependence on $N_{o P}$ (Doll et al., 2015a). That is $\left(N_{A}-1\right) \delta_{A}=0.4 \mathrm{~mA}$ when $N o P=1$; and $\left(N_{A}-1\right) \delta_{A}=0.2 \mathrm{~mA}$ when $N_{o} P=2$. Third, we consider the dependence of the step size for the reference point on $N_{o P}$. Then, we set $\Delta_{B}=0.1 \mathrm{~mA}$ and $\Delta_{B}=0.05 \mathrm{~mA}$, when $N o P=1$ and $N o P=2$, respectively. Also, for APP, we set boundaries $B^{l}=0$ and $B^{u}$ according to its dependence on $A^{u}, N_{A}, \Delta_{B}$ and $\delta_{A}$, see Eq. (5.2). There are still two remaining parameters: $N_{A}$ and $N_{S R P}$, which we will specify for various simulation cases. 


\subsubsection{Simulations of asymptotic sampling distributions}

In section 5.3, we formulated Markov models and derived their properties including asymptotic sampling distributions for general cases. Here, based on the $\mathrm{HM}$ for the nociceptive detection task, we simulate specific staircase procedures to understand properties of procedures.

First, we study the probability vector $\boldsymbol{\Phi}$ and the distribution $\boldsymbol{\pi}^{\mathbb{A}}$ for various cases. We choose $N_{A}=1,5$ and 10 . When $N_{A}=1$, we have $\boldsymbol{\Phi}=\boldsymbol{\Psi}$, indicating the APP is restricted to the SSP. Here, as an example, we use $\boldsymbol{\theta}^{(1)}$ for model parameters and $T S$ for temporal properties, see Table 5.1 and 5.2, respectively. Given these, we show various vectors of probabilities $\boldsymbol{\Phi}$ in four subplots see Fig. 5.4 IA and IB for the two combinations of temporal properties $(N o P=1, P W=0.42 \mathrm{~ms})$ and $(N o P=2$, $I P I=50 \mathrm{~ms}, P W=0.42 \mathrm{~ms})$, respectively. The filled markers indicate a pair of reference points, $b_{m}$ and $b_{m+1}$ with probabilities nearest to 0.5 , i.e. $\Phi_{m} \leq 0.5<$ $\Phi_{m+1}$. We plot distributions $\boldsymbol{\pi}^{\mathbb{B}}$ in Fig. 5.4 IIA and IIB. Also, we plot stationary distribution $\boldsymbol{\pi}^{\mathbb{A}}$ in Fig. 5.4 IIIA and IIIB. In these two panels, we use filled markers to indicate the most sampled reference points or applied amplitudes, receptively. In addition, Fig. 5.4 IVA and IVB show the logit-transformed detection probabilities, for which more details will be provided in section 5.5. For more examples, see Appendix A.

Second, we demonstrate the aliasing effect in the stationary distribution of applied amplitudes. This results in a multimodal distribution $\pi^{\mathbb{A}}$ as shown in Fig. 5.5A with model parameters $\boldsymbol{\theta}^{(1)}, N o P=1, P W=0.42 \mathrm{~ms}$, and $N_{A}=4$. Although the stationary distribution $\pi^{\mathbb{B}}$ is unimodal (see Fig. 5.5B), the assigning rule introduces aliased applied amplitudes (see Fig. 5.5C), where the size of the circle represents the product probability $\boldsymbol{\pi}_{j}^{\mathbb{B}} / N_{A}$.

Third, we illustrate the equivalence of the sampling distribution to the stationary distribution. We set six values of the number $N_{A}=1,2,4,5,7$, and 9. With model parameters $\boldsymbol{\theta}^{(1)}, N o P=1$ and $P W=0.42 \mathrm{~ms}$, we show model-simulated $\pi^{\mathbb{A}}$ in Fig. 5.6. For the asymptotic sampling distribution, we run one staircase procedure to collect $N_{S R P}=500000$ SRPs. We find that when $N_{A}=1$ or 5 , the distribution is unimodal; when $N_{A}=2,4,7$, or 9 , the distribution has multiple local peaks. The stationary distribution, shown in Fig. 5.6C is identical to the bar chart in Fig. 5.5A. Among these six cases, we remark that when $N_{A}=1$, i.e. for the SSP, the distribution has the most densely sampling distribution around the true 

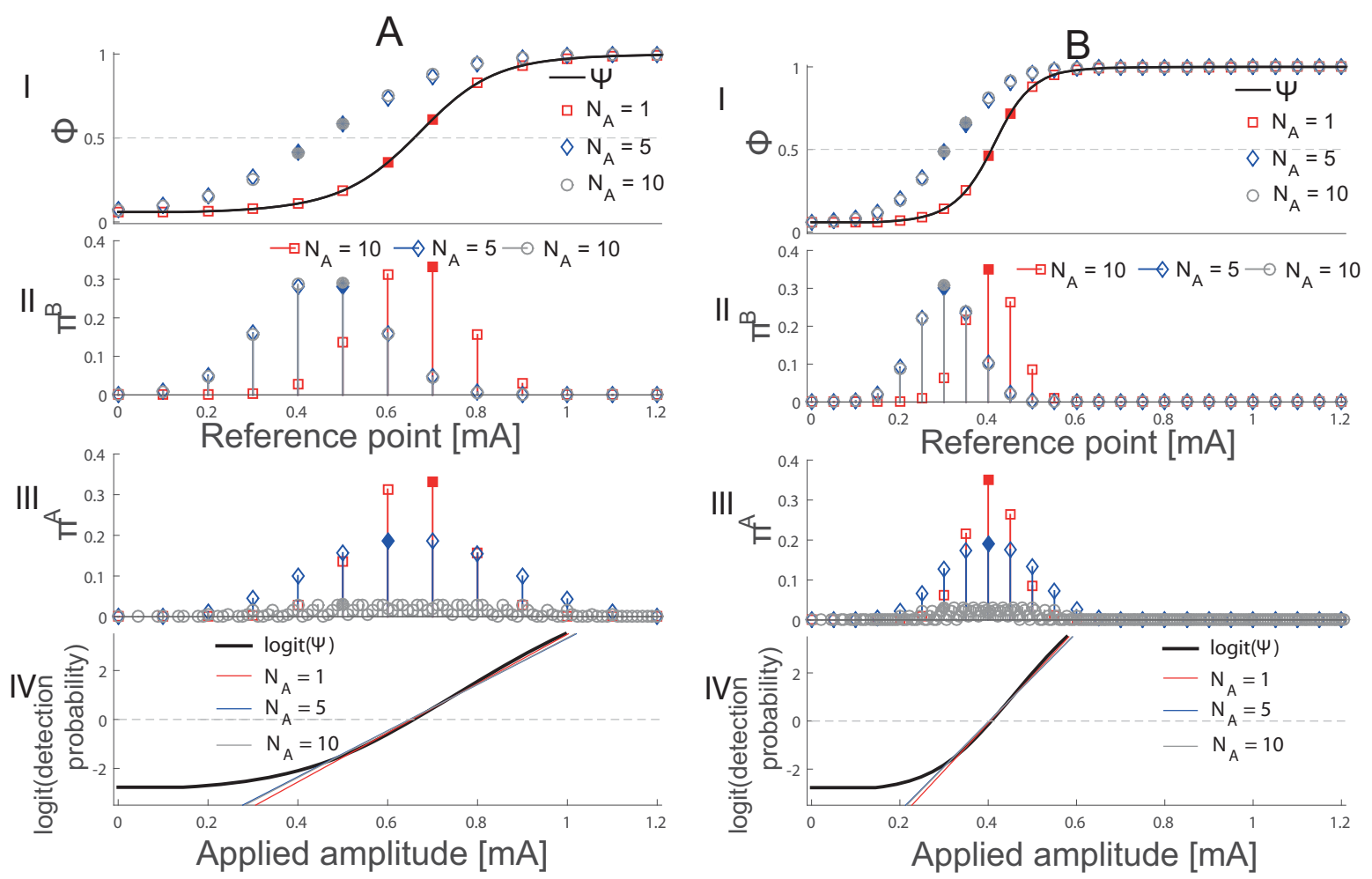

Figure 5.4: Transition probability and asymptotic characteristics for $\boldsymbol{\theta}^{(1)}$ using TS. I: $\boldsymbol{\Phi}$ for the reference points, the filled markers are a pair of points with the nearest probabilities to 0.5 , II: the stationary distribution $\pi^{\mathbb{B}}$ of reference point, the filled markers are the most frequently sampled of distributions III: the stationary distribution $\boldsymbol{\pi}^{\mathbb{A}}$ of applied amplitude, the filled markers are the most frequently sampled of distributions, IV: the logit-transformed detection probability and the best fit with logistic curve. A: NoP $=1$ and $P W=0.42 \mathrm{~ms}, \mathrm{~B}: N o P=2, I P I=10 \mathrm{~ms}$ and $P W=0.42 \mathrm{~ms}$.

detection threshold (based on the hazard model).

\subsection{Threshold estimates for staircase procedures and their statistical biases}

In this section, we investigate procedural effects on estimated thresholds. We start with a brief description of the commonly used logistic regression to estimate the detection threshold. Next, we determine asymptotic estimates of detection thresholds from logistic regression using HM-simulated SRPs. Then, we assess the bias of asymptotic estimates. After that, we perform Monte Carlo simulations to evaluate threshold estimates with finite-sample stimulus-response pairs. 


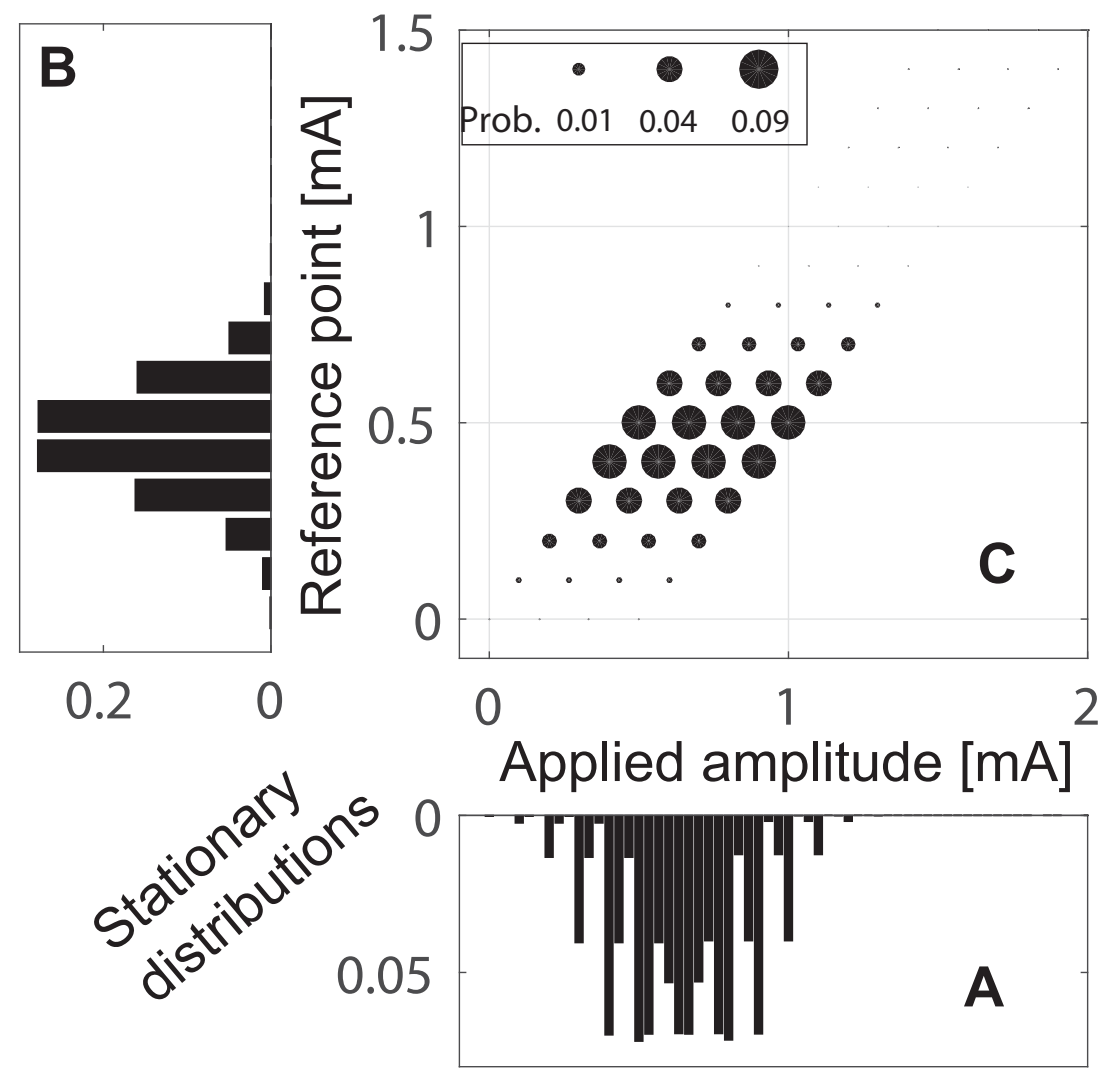

Figure 5.5: Illustration of the aliasing effect on the distribution of applied amplitudes with four modes. We set model parameters as $\boldsymbol{\theta}^{(1)}$, and temporal properties of stimuli as $N o P=1, P W=0.42 \mathrm{~ms}$, and the procedural parameter as $N_{A}=4$. A: unimodal stationary distribution of applied amplitudes $\pi^{\mathbb{A}}$, B: multimodal stationary distribution of Reference points $\boldsymbol{\pi}^{\mathbb{B}}$, C: $2 \mathrm{D}$ representation of $\boldsymbol{\pi}^{\mathbb{B}}$ and its transformation to $\boldsymbol{\pi}^{\mathbb{A}}$, where the area of the circle indicates the probability.

\subsubsection{Logistic regression}

The logistic regression is used to model the relation between the detection probability and the stimulus amplitude (Doll et al., 2014). The logit-transformed detection probability is given by a linear function of applied amplitude $A$,

$$
\operatorname{logit}(\operatorname{Pr}(R=1 \mid A)):=\log \left(\frac{\operatorname{Pr}(R=1 \mid A)}{\operatorname{Pr}(R=0 \mid A)}\right)=\beta_{0}+\beta_{1} A,
$$

where $\beta_{0}$ and $\beta_{1}$ are two regression coefficients, interpreted as the intercept and slope of the straight line. We give $\boldsymbol{\beta}:=\left(\beta_{0}, \beta_{1}\right)$. This linearization technique permits to 

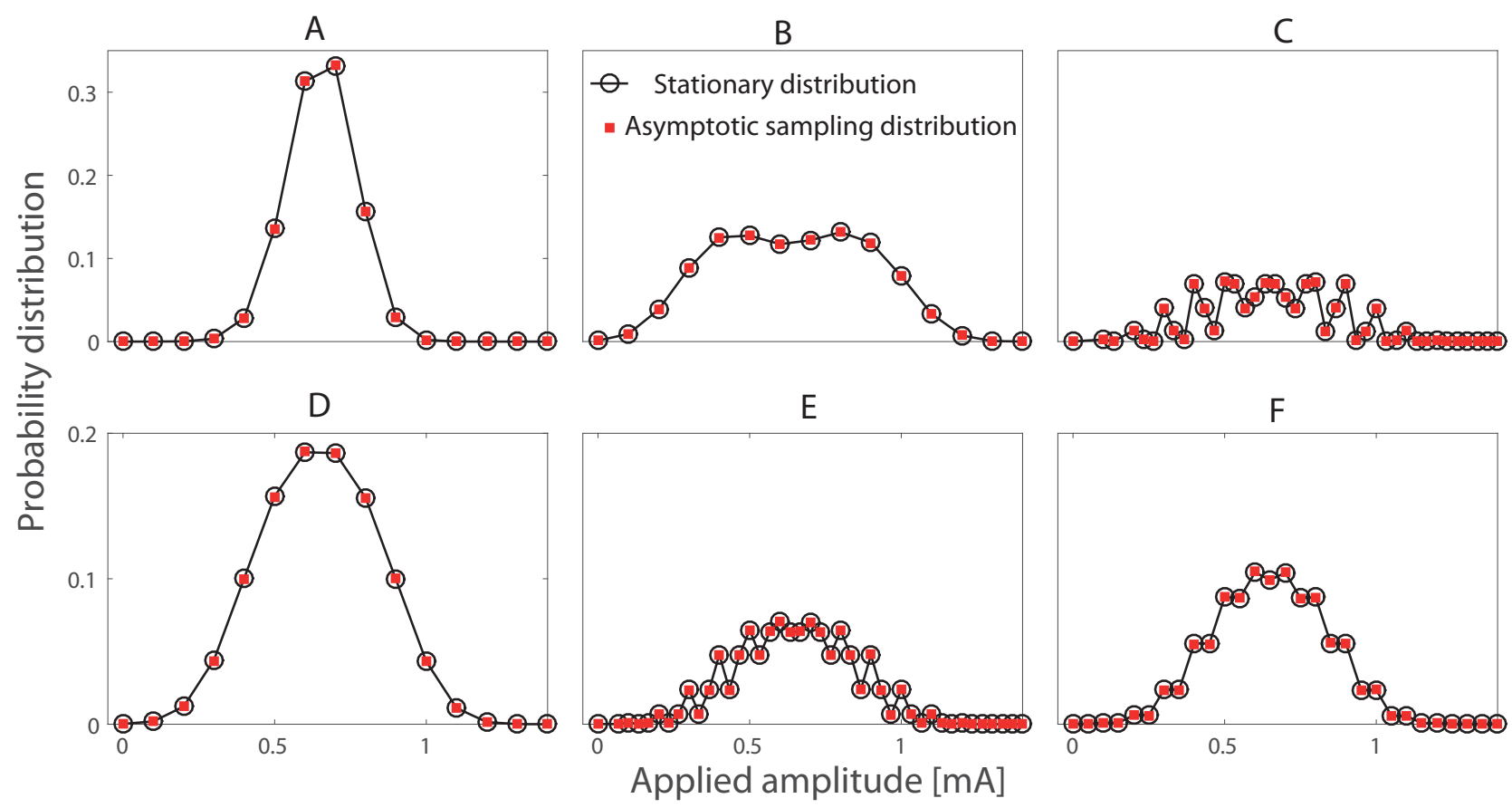

Figure 5.6: Asymptotic sampling distributions (red squares) with $N_{S R P}=500000$ and stationary distributions (black circles) of Markov models. We set $N o P=1$ and $P W=$ 0.42 ms. Panels A-F correspond to the cases when $N_{A}=1,2,4,5,7$, and 9 , respectively.

straightforwardly address the inverse problem, i.e. from a set of stimulus-response pairs to estimates of coefficients $\hat{\beta}_{0}$ and $\hat{\beta}_{1}$. Then, one can determine detection threshold $\hat{\alpha}_{50}:=-\frac{\hat{\beta}_{0}}{\hat{\beta}_{1}}$.

Simulation studies of the bias begin with generating $N_{m}$ realizations of sets of SRPs. Then, one determines a set of $N_{m}$ threshold estimates from regression. The variability among the set of $N_{m}$ estimated thresholds results from the stochastic nature of realizations containing SRPs. To evaluate the performance of logistic regression, one computes the bias of estimated thresholds by

$$
B_{\alpha}=\frac{1}{N_{m}} \sum_{k=1}^{N_{m}}\left(\hat{\alpha}_{50}^{k}-\alpha_{50}^{t}\right),
$$

where $k$ is the index of the realization dataset and the realization number $N_{m}$ is set as 10000 .

\subsubsection{Asymptotic characteristics of threshold estimates}

Now, we are ready to assess the estimation performance to obtain thresholds using logistic regression. Given that when $N_{S R P} \rightarrow \infty$, the asymptotic sampling 
distribution converges to the unique stationary distribution of the Markov model in section 5.4 or illustrative examples in Fig. 5.6. Together with the logistic regression, one can expect that the variability of resultant estimates also vanishes. In the asymptotic situation, we determine regression coefficients via the following weightedleast-squares estimation

$$
\hat{\boldsymbol{\beta}}^{\infty}:=\operatorname{argmin}_{\boldsymbol{\beta}} \sum_{i=1}^{n(\mathbb{A})}\left(\left(\left(\beta_{0}+\beta_{1} a_{i}\right)-\operatorname{logit}\left(\Psi_{i}\right)\right) \boldsymbol{\pi}_{i}^{\mathbb{A}}\right)^{2} .
$$

Then, we give the asymptotic threshold estimate as $\hat{\alpha}_{50}^{\infty}=-\frac{\hat{\beta}_{0}^{\infty}}{\hat{\beta}_{1}^{\infty}}$, where $\infty$ indicates the asymptotic setting.

Here, we compute asymptotic threshold estimates in various cases for the nociceptive detection task. We consider the seven vectors of model parameters in Table 5.1, four combinations in Table 5.2, and ten distinct numbers $N_{A}=1,2, \ldots, 10$. First, we check whether the logistic regression can introduce non-neglectable bias. With $N_{A}=1,5$ and 10, we plot logit-transformed model-based psychometric functions and corresponding fitted logistic curves, see Fig. 5.4IV. We remark the mismatch between these two kinds of curves results in different detection thresholds. Second, we assess the effect of $N_{A}$ on the bias term $\hat{\alpha}_{50}^{\infty}-\alpha_{50}^{t}$. We compute the total $7 \times 4 \times 10$ studied cases, yielding 280 biases of detection thresholds. We show results for five vectors of values of model parameters in Fig. 5.7. The other two's results are similar (not shown for $\boldsymbol{\theta}^{(2)}$ and $\boldsymbol{\theta}^{(6)}$ ). Based on the results, one could see that, with fixed model parameters $\boldsymbol{\theta}$, there is one case with negative biases. For the remaining cases, the logistic regression results in overestimated detection thresholds. Also, biases in Fig. 5.7 A, B are larger than those in $\mathrm{C}$ and $\mathrm{D}$ even with same values of model parameters which is due to larger values of $\left(N_{A}-1\right) \delta_{A}$ and $\Delta_{B}$ for $N o P=1$. Third, we compute 280 relative biases with respect to the corresponding true thresholds, $\epsilon_{r}:=\frac{\hat{\alpha}_{50}^{\infty}-\alpha_{50}^{t}}{\alpha_{50}^{t}}$. Then, we plot the empirical distribution of the relative bias in Fig. 5.8. One can find that about $40 \%$ cases with a relative bias higher than $10 \%$. In addition, the distribution has a majority of 280 cases with positive biases, indicating that logistic regression is much more likely to overestimate the detection threshold.

From above simulation results, we summarize that first, an APP can introduce more bias than the SSP. Second, in most cases where $N_{A}>1$, the larger $N_{A}$, the smaller the absolute value of the bias. However, for cases with $\boldsymbol{\theta}^{(5)}$, we notice fluctuations of biases with increased values of $N_{A}$ in Fig. $5.7 \mathrm{~A}$ and B. We will 


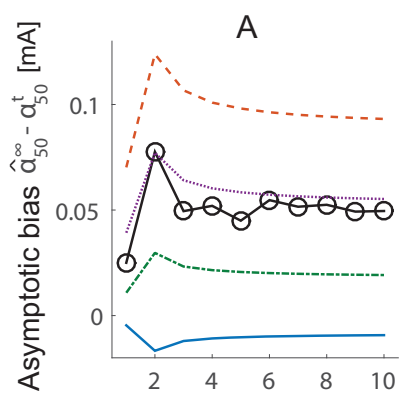

B

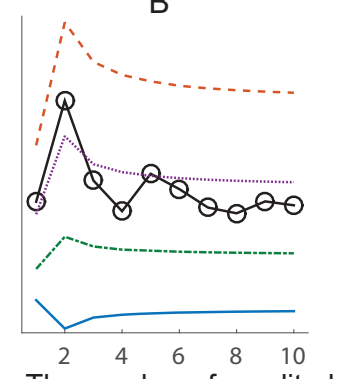

The number of amplitudes in the moving set: $N_{A}$
$\mathrm{D}$

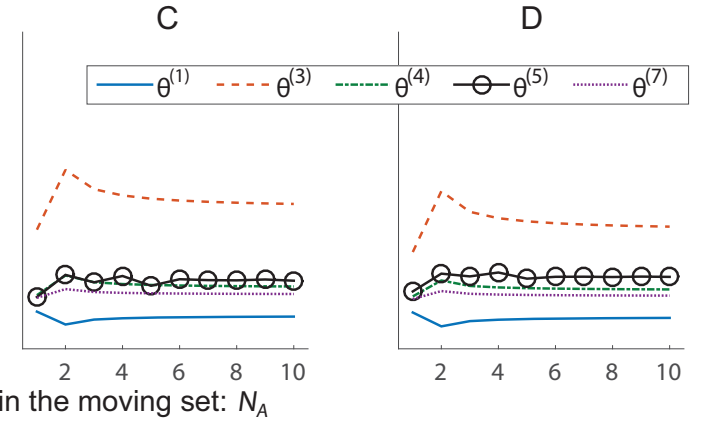

Figure 5.7: Effects of $N_{A}$ on the asymptotic bias of threshold estimates for five different cases of model parameter vectors. In total, we use four combinations of temporal stimulus properties in $T S$.

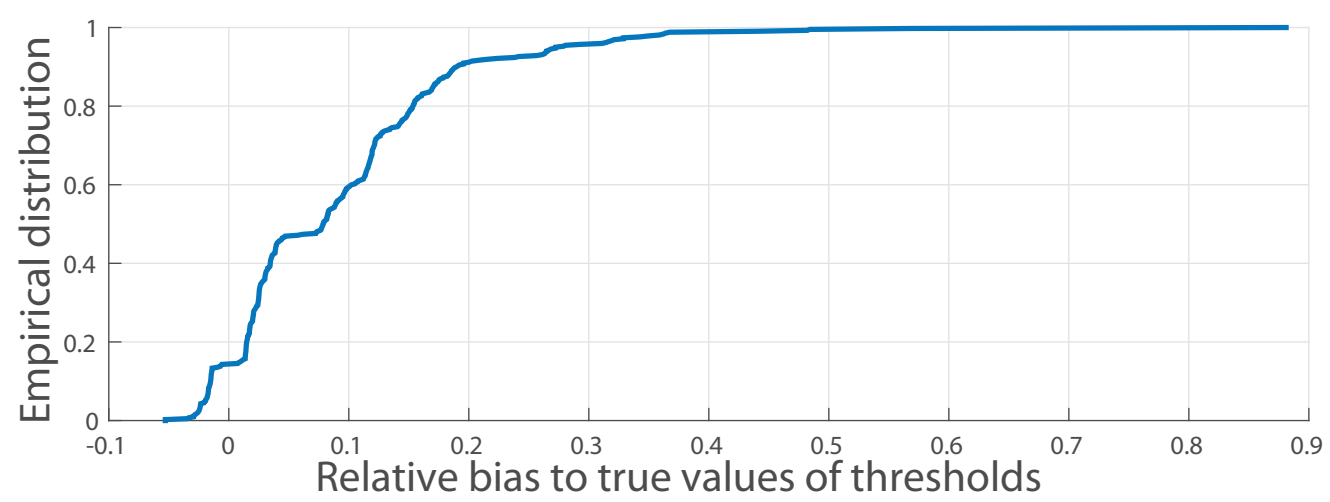

Figure 5.8: Empirical distribution of the relative bias of asymptotic threshold estimates. We consider various values of $N_{A}$, temporal properties and values of model parameters.

discuss later. Third, the biases have the same signs across different combinations of temporal stimulus properties.

\subsubsection{Estimation of thresholds with finite samples of stimulus- response pairs}

Now, we study the estimation performance, when the pairs of stimuli and responses are finite. We employ various values of the number of stimulus-response pairs $N_{S R P}=50,100,500$, and $5 \times 10^{6}$. We determine a set of $N_{m}=10000$ estimates of detection threshold from Monte Carlo simulations. Then, we compute a set of $N_{m}$ corresponding differences, $\left\{\hat{\alpha}_{50, k}-\alpha_{50}^{t}\right\}$, with $k \in\left\{1,2, \ldots, N_{m}\right\}$. To assess the estimation performance, we look at the empirical distribution of those differences. We implemented the Monte-Carlo simulations in MATLAB 2014b, where we used 
the GLMFIT route to perform logistic regression. As an ideal situation, the empirical distribution will be a step function at zero, which indicates to obtain the true value of the detection threshold almost surely. However, both the variability from realizations and mismatch of the underlying and estimation psychometric function could hamper the estimation performance, resulting in a shallower curve. Here, we exemplify the distribution by fixing $\boldsymbol{\theta}=\boldsymbol{\theta}^{(6)}$. Fig. 5.9 shows these representative distributions of those differences using logistic regression, where plane A stands for $N o P=1$ and $P W=0.42 \mathrm{~ms}$; and plane B stands for $N o P=2, I P I=10 \mathrm{~ms}$ and $P W=0.42 \mathrm{~ms}$. Label I, II and III indicate values of the procedure parameter $N_{A}=1,5$, and 10, respectively. Given the set of determined thresholds, one could

A
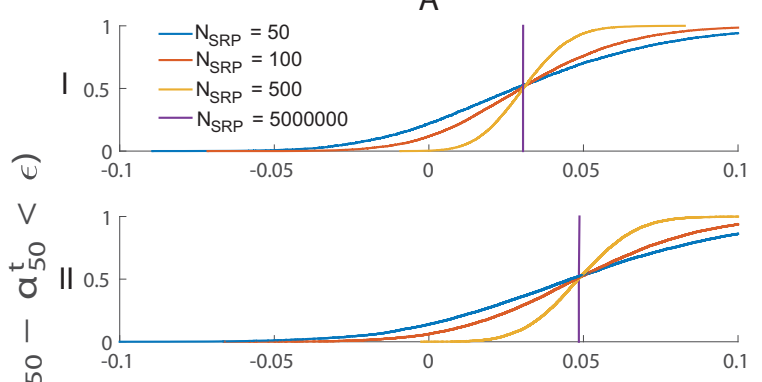
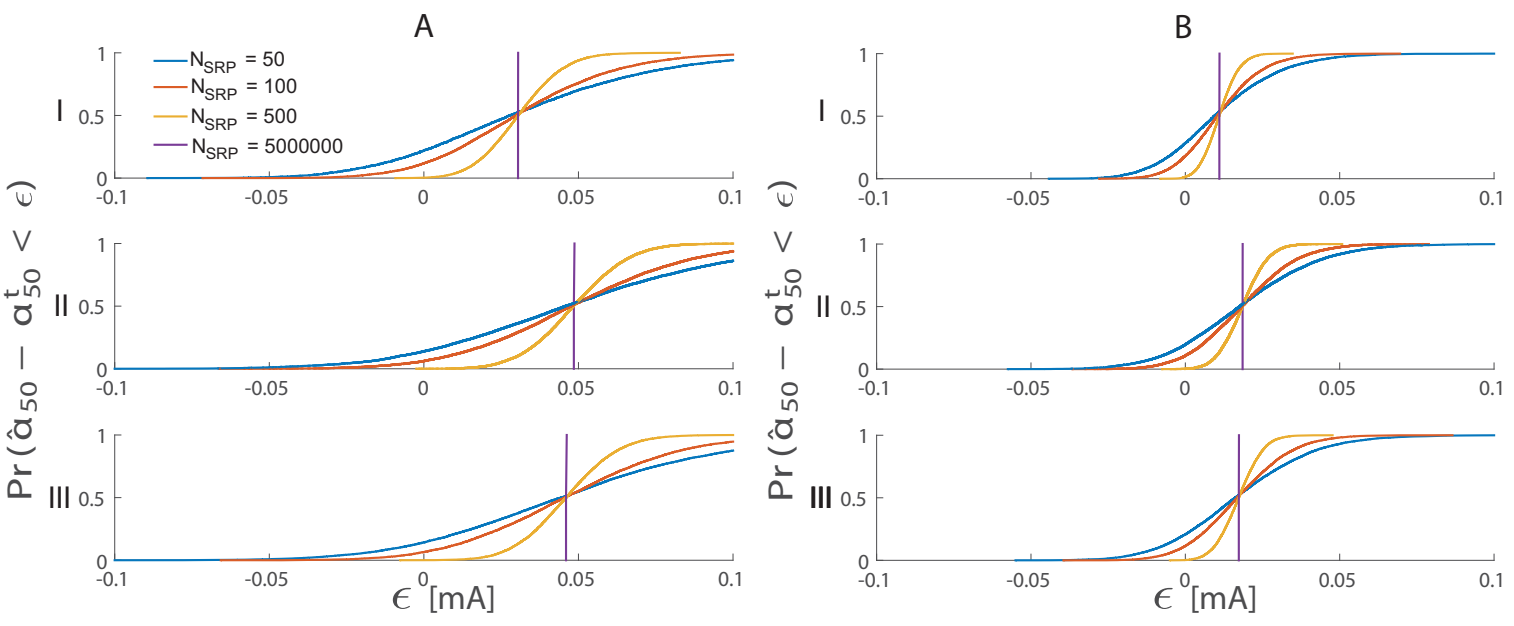

Figure 5.9: Estimation performance of detection thresholds estimated from logistic regression for the case when model parameter values $\boldsymbol{\theta}^{(6)}$ and two different combinations of temporal stimulus properties. Each panel shows empirical distributions of the errors, the difference between estimated and true thresholds. A: $N o P=1, P W=0.42 \mathrm{~ms}$ and B: $N o P=2, I P I=10 \mathrm{~ms}, P W=0.42 \mathrm{~ms}$. We consider three different values of $N_{A}=1,5$ and 10 and show the results in panels labeled by I, II and III, respectively.

compute the bias according to (5.22). However, such an extracted characteristic may not represent the estimation performance, as $\hat{\alpha}_{50}-\alpha_{50}^{t}$ could be non-normally distributed. Hence, the empirical distribution should be more representative of the estimation performance. The extensive summary of results for other parameter values and temporal stimulus properties are reported in Appendix B. 


\subsection{Conclusions and discussion}

We have formulated a recently developed staircase procedure, i.e. the adaptive probing procedure for an experimental detection task (Doll et al., 2014, 2015b), as a Markov model. This formulation is a generalization of the Markov chain which is merely for the simple staircase procedure. Based on this formulation, we have shown the equivalence of the asymptotic sampling distribution to the stationary distribution of the Markov model. In addition, we show how the procedure affects the sampling distribution in asymptotic cases. In cases of finite stimulus-response pairs, our simulation results also show that SSP has smaller biases of estimated thresholds than APP.

First, for the same nociceptive detection task with electrocutaneous stimulation, the previous simulation-based study (Doll et al., 2014) reported only negligible bias in estimated thresholds using logistic regression. However, our result shows above $40 \%$ cases with relative biases larger than $10 \%$, see Fig. 5.8. We remark that this difference can be attributed to the mismatch between the underlying and estimation psychometric functions. With our Markovian formulation, in asymptotic situations, we consider logistic regression as a weighted least square method to determine coefficients, see Eq. 5.23. This explains that when no mismatch between curves, one can expect unbiased estimates of regression coefficients and detection thresholds, as a logistic curve is a generalized linear model (Conceptual understanding could be "any two points of a line determine the same line"). With non-negligible mismatch, sampling distribution of applied amplitude affects the estimation results. With properties of Markov models, we argue that the SSP could sample more densely around the detection thresholds than the APP. This yields the better performance of the SSP in the estimated thresholds, shown in various groups of simulations.

Second, we notice that the step size $\Delta_{B}$ is an important influential factor for the bias of threshold estimates. A relatively small value of $\Delta_{B}$ could justify the usage of the logistic regression for a justified approximation of the different form of the psychometric function. We argue this by revisiting simulation results in Fig. 5.7. When $N o P=1$ and $P W=0.84 \mathrm{~ms}$, the steepness of psychometric function is expected to be comparable with those for the double pulse stimuli with $P W=0.42 \mathrm{~ms}$. When $N o P=2$, we set $\Delta_{B}=0.05 \mathrm{~mA}$ which is half of that for $N o P=1$. Comparing results in Fig. $5.7 \mathrm{~B}$ to either $5.7 \mathrm{C}$ or $\mathrm{D}$, a larger value of $\Delta_{B}$ resulted in more bias of detection thresholds in the asymptotic setting. In case 
of finite-sample SRPs, our simulation results in Appendix B also support this. In addition, we notice that in Fig. 5.7A and $\mathrm{B}$, for the cases with $\boldsymbol{\theta}^{(5)}$, the biases in APPs fluctuate as $N_{A}$ increases. Our explanation is that psychometric curves are relatively steep, see Fig. 5.20A and B in Appendix A. Together with $\Delta_{B}=0.1 \mathrm{~mA}$, it yields this fluctuation. Furthermore, with halved step sizes $\Delta_{B}=0.05 \mathrm{~mA}$ for $N o P=2$, we only found decreased bias with increased $N_{A}$ from 2 to 10 rather than fluctuation, see Fig. 5.7C and D.

Last, our Markovian formulation provides theoretical support in an assessment of statistical performance of threshold estimates. In more realistic situations, we acknowledge that the underlying psychometric curve could be non-stationary, e.g. (Doll et al., 2014; Naugle et al., 2015), which can violate assumptions to derive the properties of Markov model. On the other hand, this non-stationarity in the psychometric curve would further increase the mismatch between the underlying and estimation curves. Given the above-mentioned non-negligible bias for the stationary curves, one can expect further hampered estimation results for non-stationary situations. Our simulations show that the applied amplitudes are more widely distributed for APP than SSP. Also, multiple modes can exist in the asymptotic distribution with APP. This may imply that in a non-stationary situation, APP could be more robust than the SSP in estimating time-varying thresholds.

\section{Appendix A: Summary of asymptotic sampling dis- tribution of applied amplitudes}

We summarize all various studied cases with simulation results on $\boldsymbol{\Phi}$, distribution $\pi^{\mathbb{A}}$ and the mismatch between model-based and fitted logistic curves. We present results in Fig. 5.10-5.16, for seven parameter vectors from $\theta^{(1)}$ to $\theta^{(7)}$. 

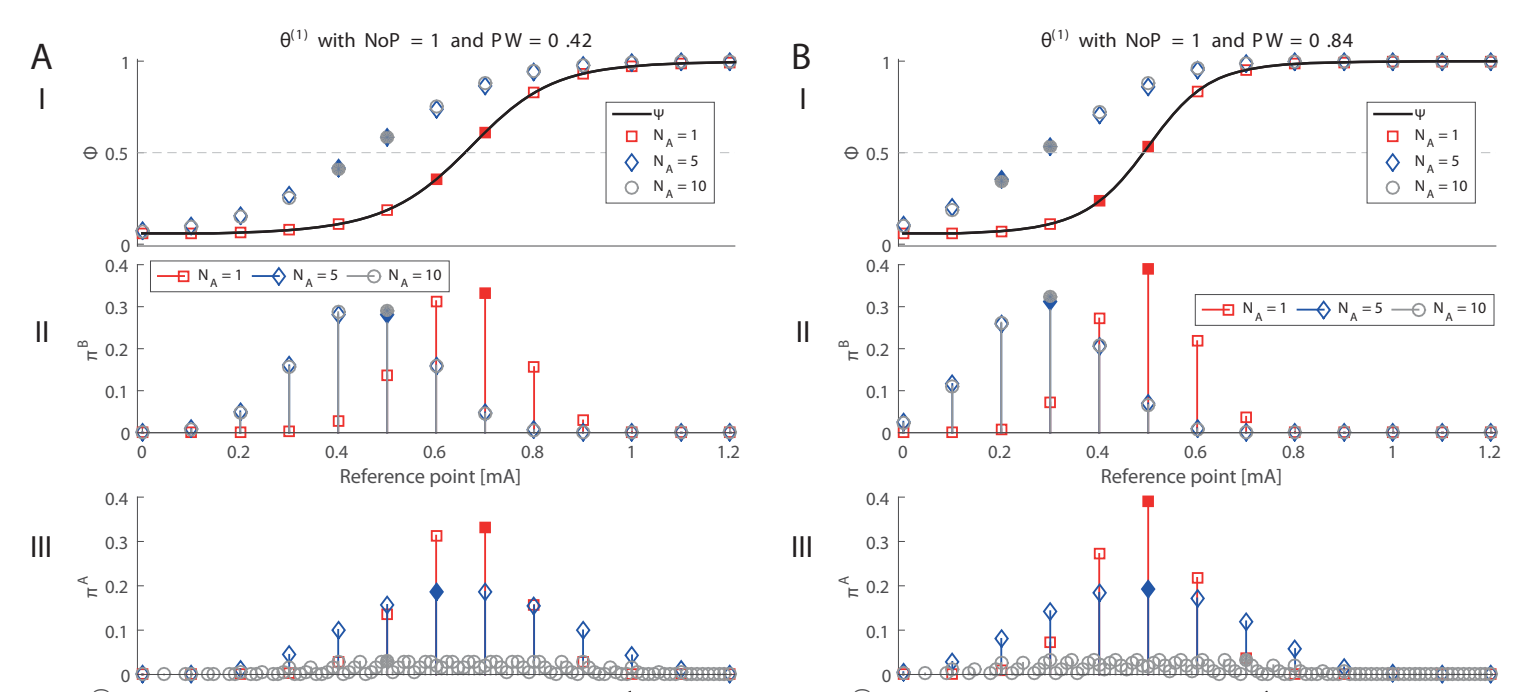

IV

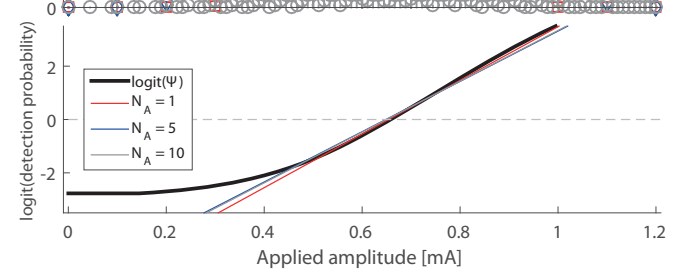

IV
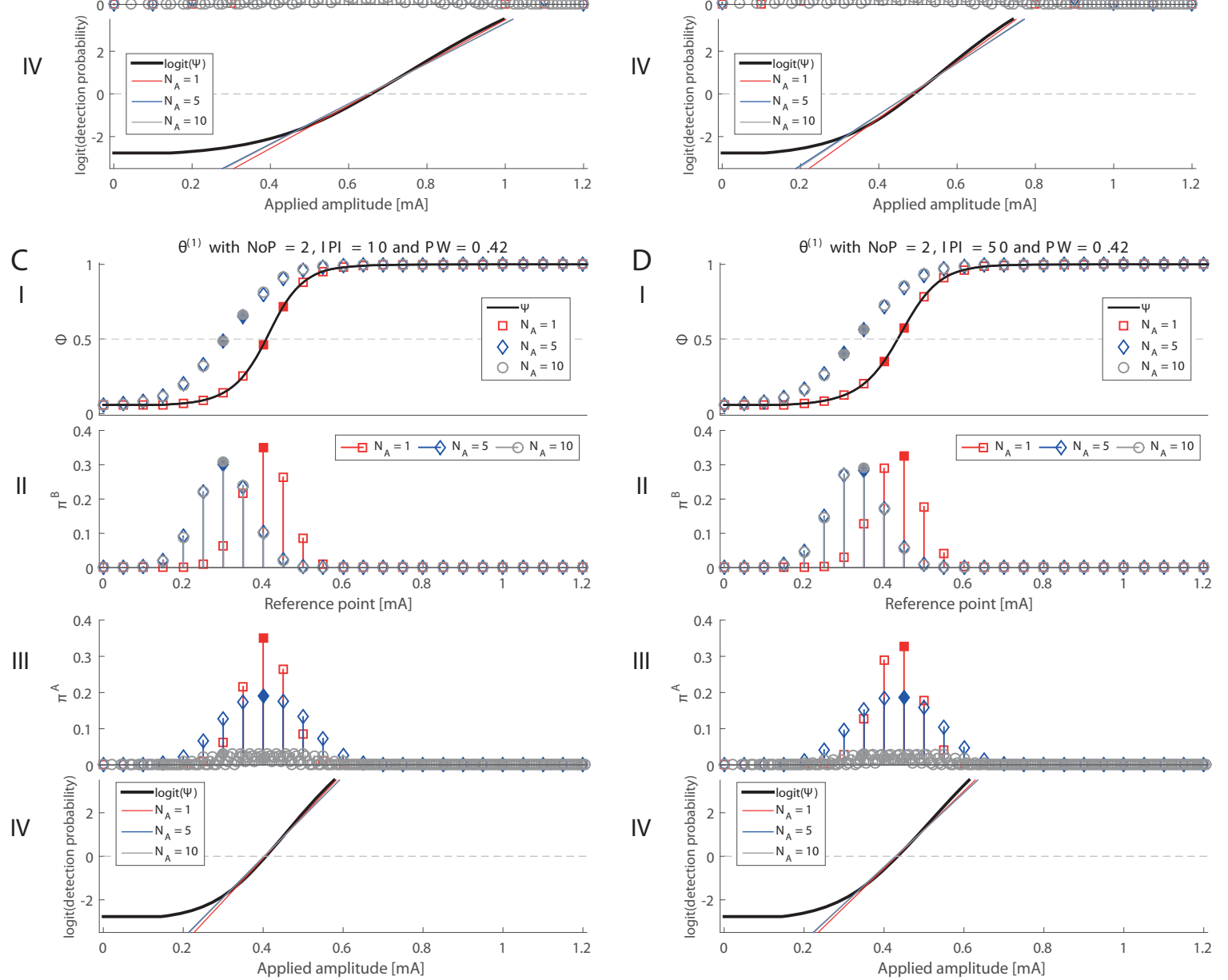

Figure 5.10: Transition probability and asymptotic characteristics for the case with $\boldsymbol{\theta}^{(1)}$ and TS. Panel A-D indicate combination A-D of TS in Table 5.2. I: $\boldsymbol{\Phi}$ for the reference points, the filled markers are a pair of points with the nearest probabilities to 0.5 , II: the stationary distribution $\pi^{\mathbb{B}}$ of reference point, the filled markers are the most frequently sampled of distributions III: the stationary distribution $\pi^{\mathbb{A}}$ of applied amplitude, the filled markers are the most frequently sampled of distributions, IV: the logit-transformed detection probability and the best fitted logistic curve. 

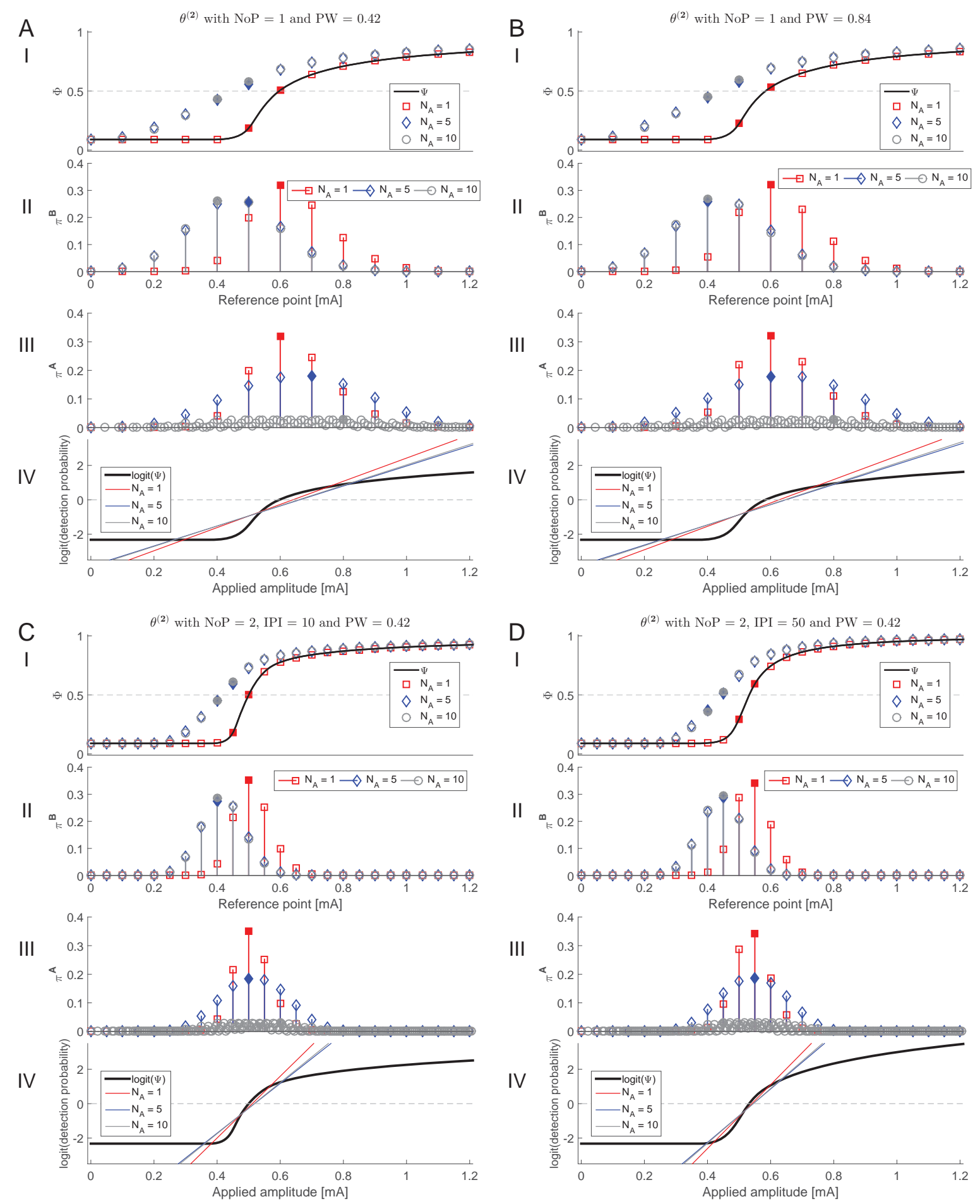

Figure 5.11: Transition probability and asymptotic characteristics for the case with $\boldsymbol{\theta}^{(2)}$ and TS. Panel A-D indicate combination A-D of $T S$ in Table 5.2. I: $\boldsymbol{\Phi}$ for the reference points, the filled markers are a pair of points with the nearest probabilities to 0.5 , II: the stationary distribution $\pi^{\mathbb{B}}$ of reference point, the filled markers are the most frequently sampled of distributions III: the stationary distribution $\boldsymbol{\pi}^{\mathbb{A}}$ of applied amplitude, the filled markers are the most frequently sampled of distributions, IV: the logit-transformed detection probability and the best fitted logistic curve. 

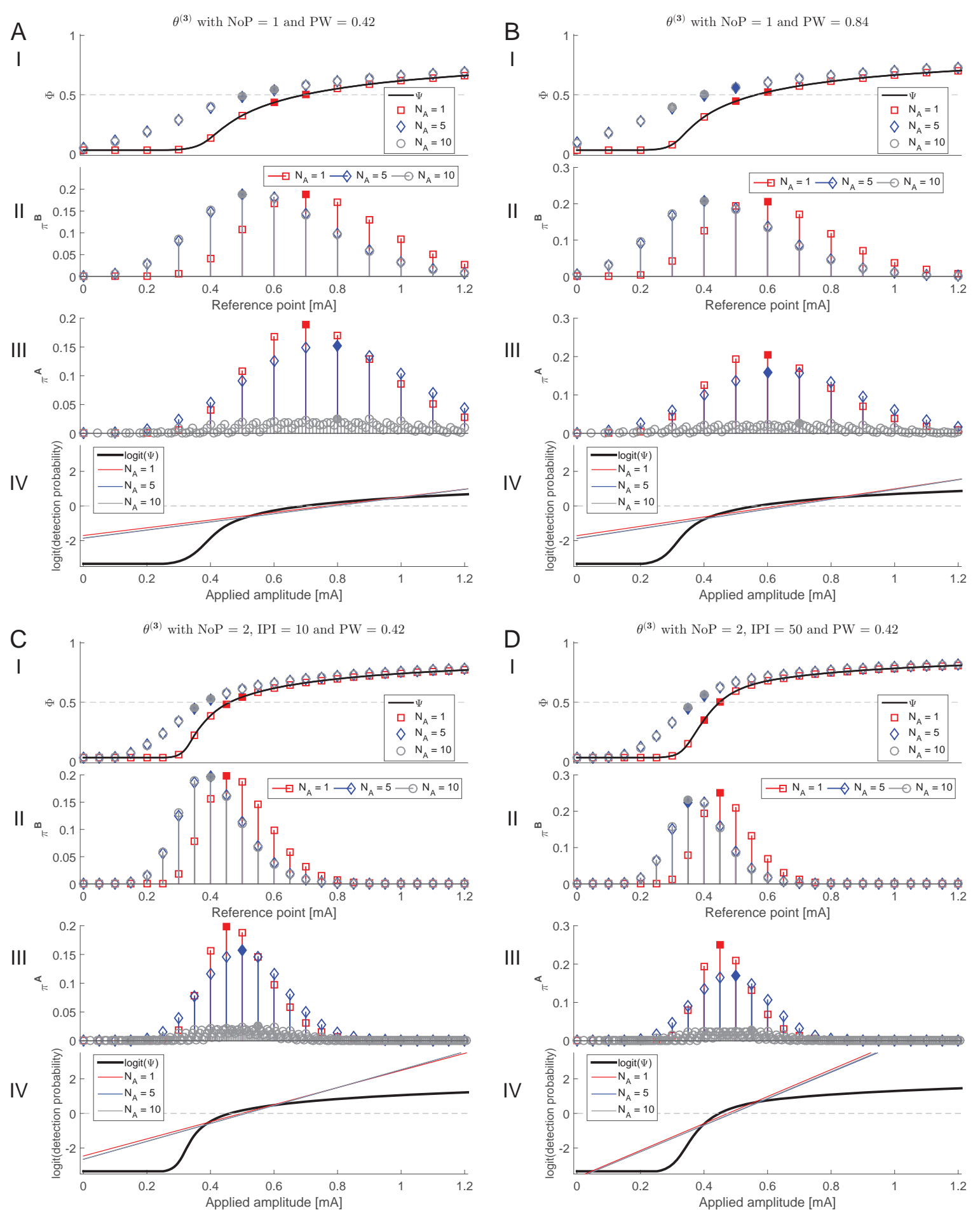

Figure 5.12: Transition probability and asymptotic characteristics for the case with $\boldsymbol{\theta}^{(3)}$ and TS. Panel A-D indicate combination A-D of TS in Table 5.2. I: $\boldsymbol{\Phi}$ for the reference points, the filled markers are a pair of points with the nearest probabilities to 0.5 , II: the stationary distribution $\pi^{\mathbb{B}}$ of reference point, the filled markers are the most frequently sampled of distributions III: the stationary distribution $\boldsymbol{\pi}^{\mathbb{A}}$ of applied amplitude, the filled markers are the most frequently sampled of distributions, IV: the logit-transformed detection probability and the best fitted logistic curve. 

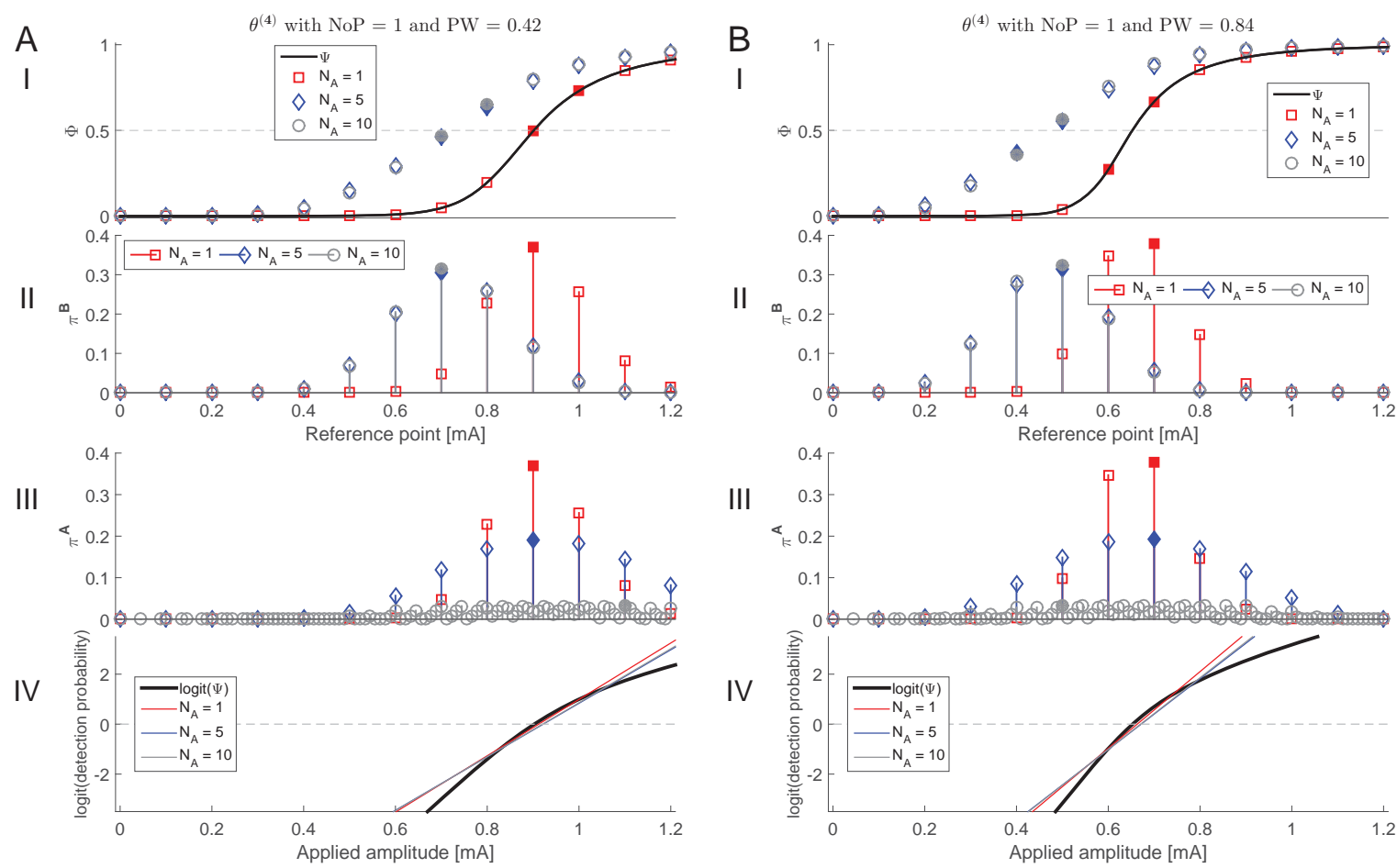

IV

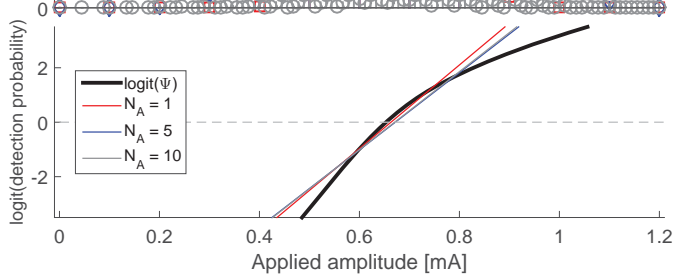

C
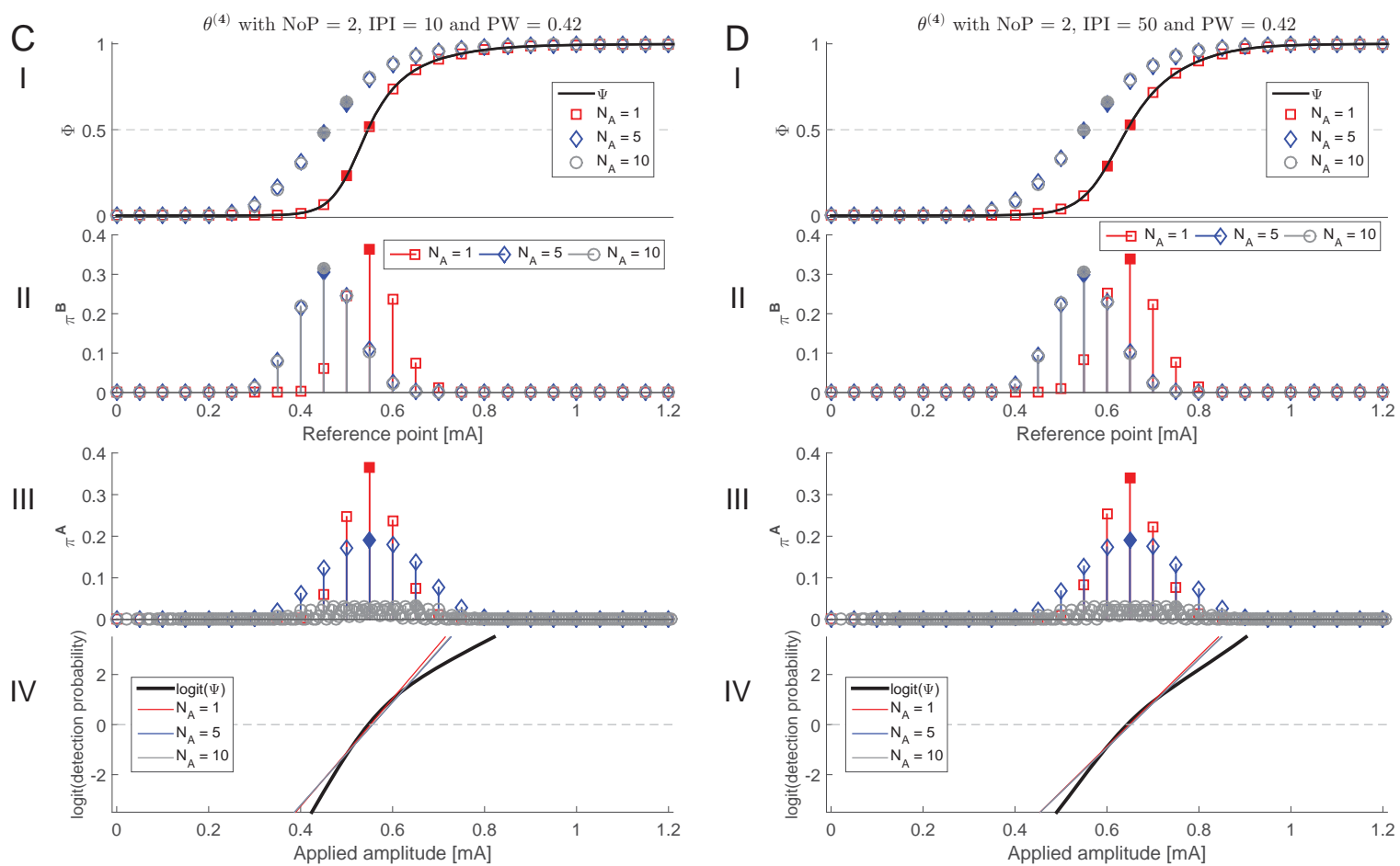

Figure 5.13: Transition probability and asymptotic characteristics for the case with $\boldsymbol{\theta}^{(4)}$ and TS. Panel A-D indicate combination A-D of $T S$ in Table 5.2. I: $\boldsymbol{\Phi}$ for the reference points, the filled markers are a pair of points with the nearest probabilities to 0.5 , II: the stationary distribution $\pi^{\mathbb{B}}$ of reference point, the filled markers are the most frequently sampled of distributions III: the stationary distribution $\pi^{\mathbb{A}}$ of applied amplitude, the filled markers are the most frequently sampled of distributions, IV: the logit-transformed detection probability and the best fitted logistic curve. 

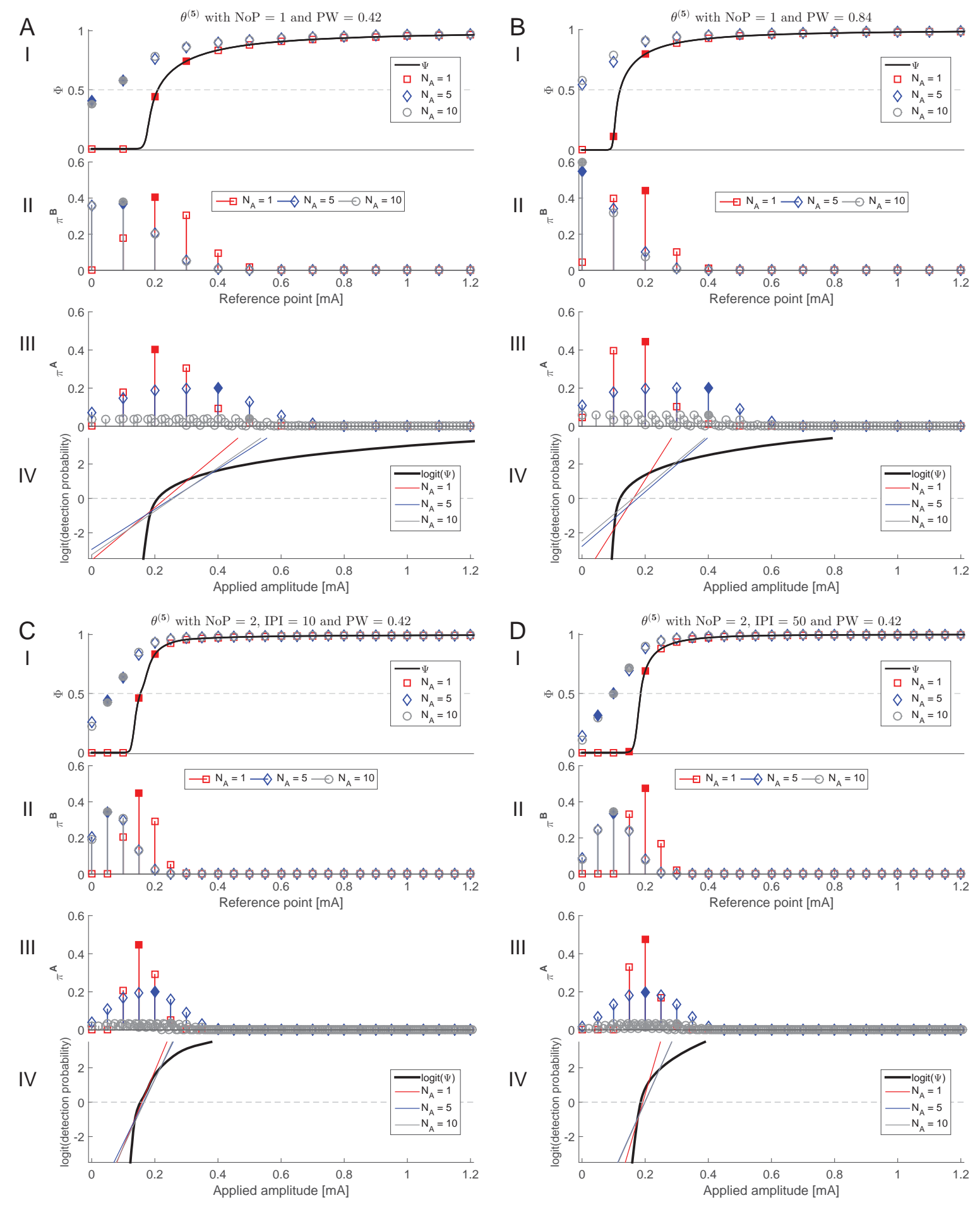

Figure 5.14: Transition probability and asymptotic characteristics for the case with $\boldsymbol{\theta}^{(5)}$ and TS. Panel A-D indicate combination A-D of TS in Table 5.2. I: $\boldsymbol{\Phi}$ for the reference points, the filled markers are a pair of points with the nearest probabilities to 0.5 , II: the stationary distribution $\pi^{\mathbb{B}}$ of reference point, the filled markers are the most frequently sampled of distributions III: the stationary distribution $\pi^{\mathbb{A}}$ of applied amplitude, the filled markers are the most frequently sampled of distributions, IV: the logit-transformed detection probability and the best fitted logistic curve. 

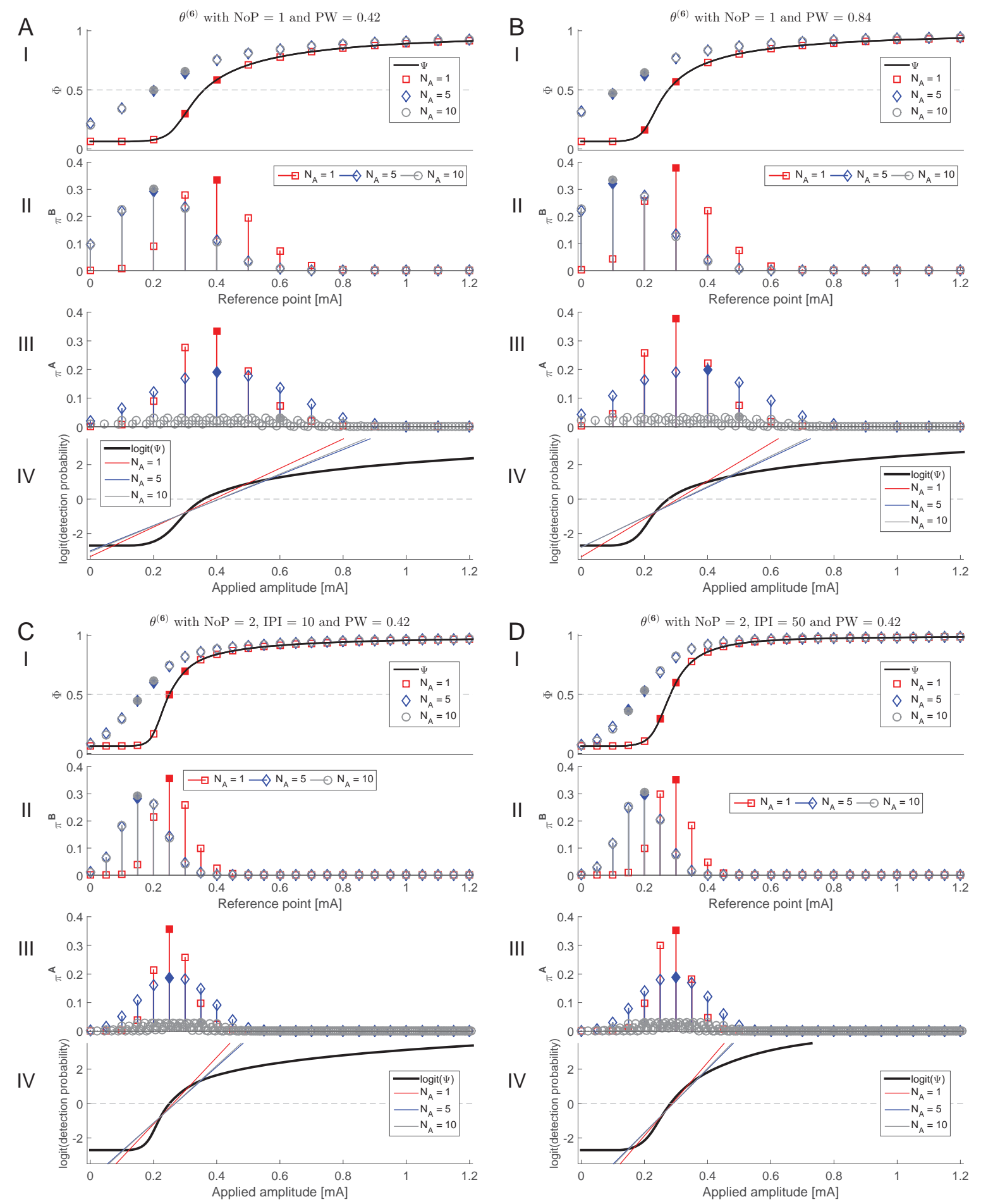

Figure 5.15: Transition probability and asymptotic characteristics for the case with $\boldsymbol{\theta}^{(6)}$ and TS. Panel A-D indicate combination A-D of $T S$ in Table 5.2. I: $\boldsymbol{\Phi}$ for the reference points, the filled markers are a pair of points with the nearest probabilities to 0.5 , II: the stationary distribution $\pi^{\mathbb{B}}$ of reference point, the filled markers are the most frequently sampled of distributions III: the stationary distribution $\boldsymbol{\pi}^{\mathbb{A}}$ of applied amplitude, the filled markers are the most frequently sampled of distributions, IV: the logit-transformed detection probability and the best fitted logistic curve. 

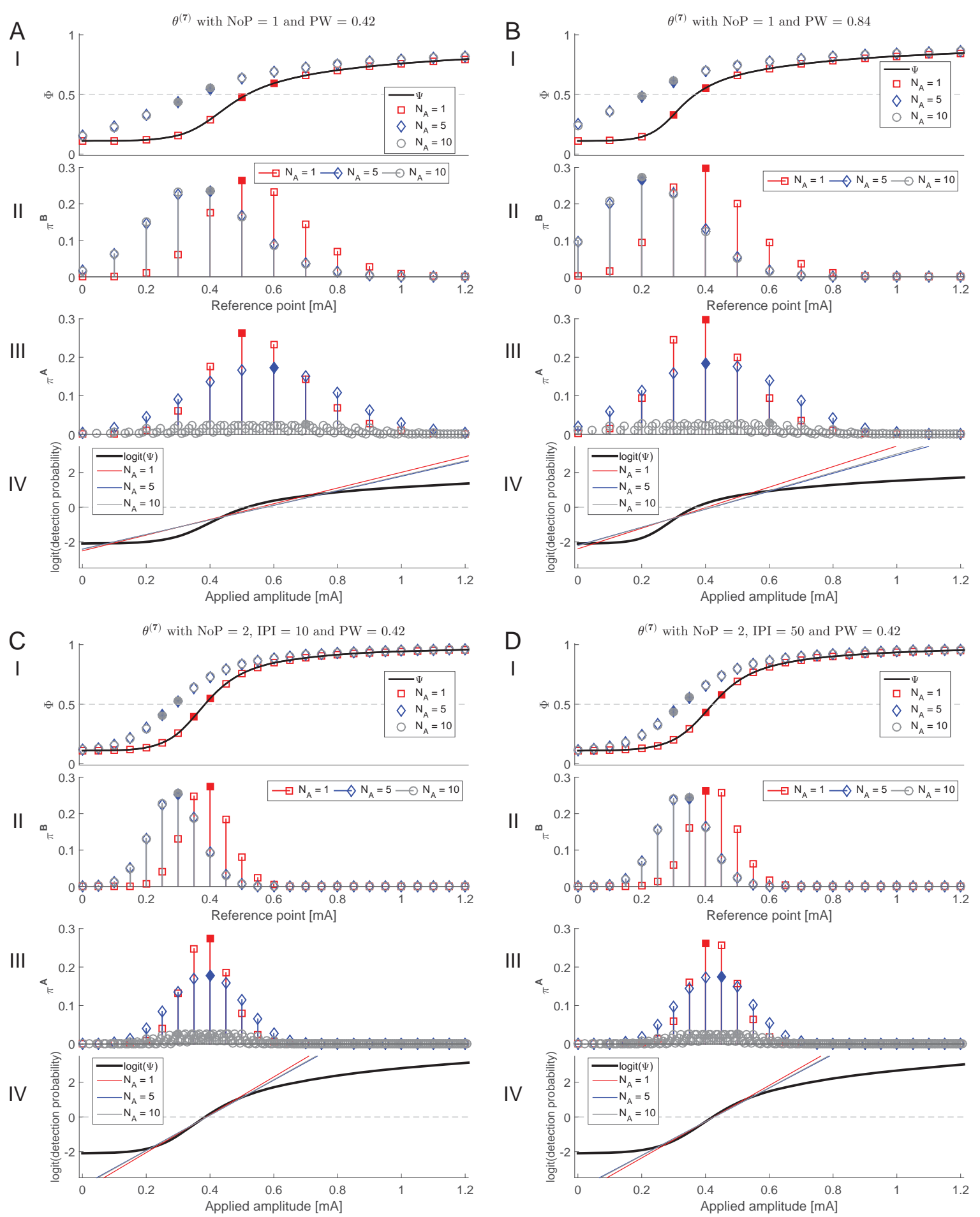

Figure 5.16: Transition probability and asymptotic characteristics for the case with $\boldsymbol{\theta}^{(7)}$ and TS. Panel A-D indicate combination A-D of TS in Table 5.2. I: $\boldsymbol{\Phi}$ for the reference points, the filled markers are a pair of points with the nearest probabilities to 0.5 , II: the stationary distribution $\pi^{\mathbb{B}}$ of reference point, the filled markers are the most frequently sampled of distributions III: the stationary distribution $\boldsymbol{\pi}^{\mathbb{A}}$ of applied amplitude, the filled markers are the most frequently sampled of distributions, IV: the logit-transformed detection probability and the best fitted logistic curve. 


\section{Appendix B: Summary of estimation performance of detection thresholds from logistic regression using various lengths of SRPs}

We summarize all studied cases with simulation results on the empirical distribution of the estimation error $\hat{\alpha}_{50}-\alpha_{50}^{t}$. We present results in Fig. 5.17-5.23, for seven parameter vectors from $\theta^{(1)}$ to $\theta^{(7)}$.
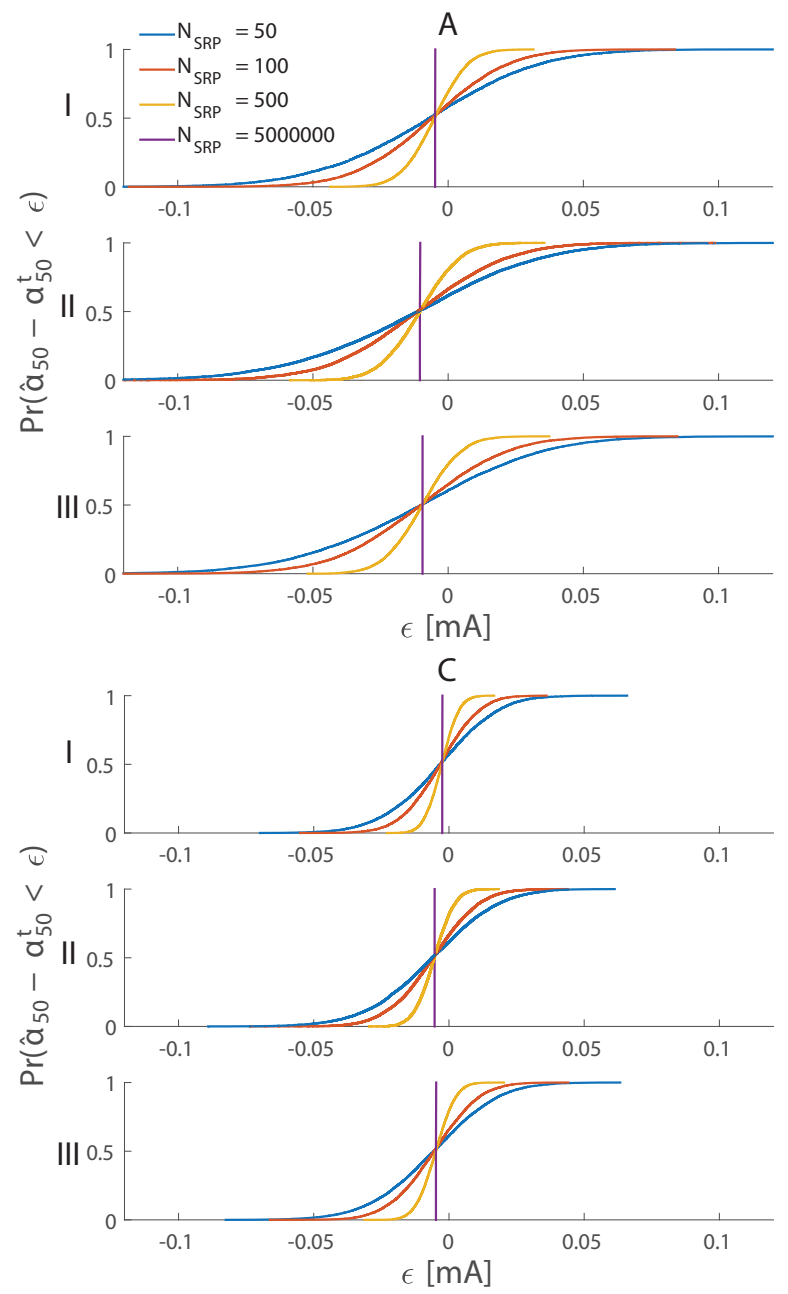
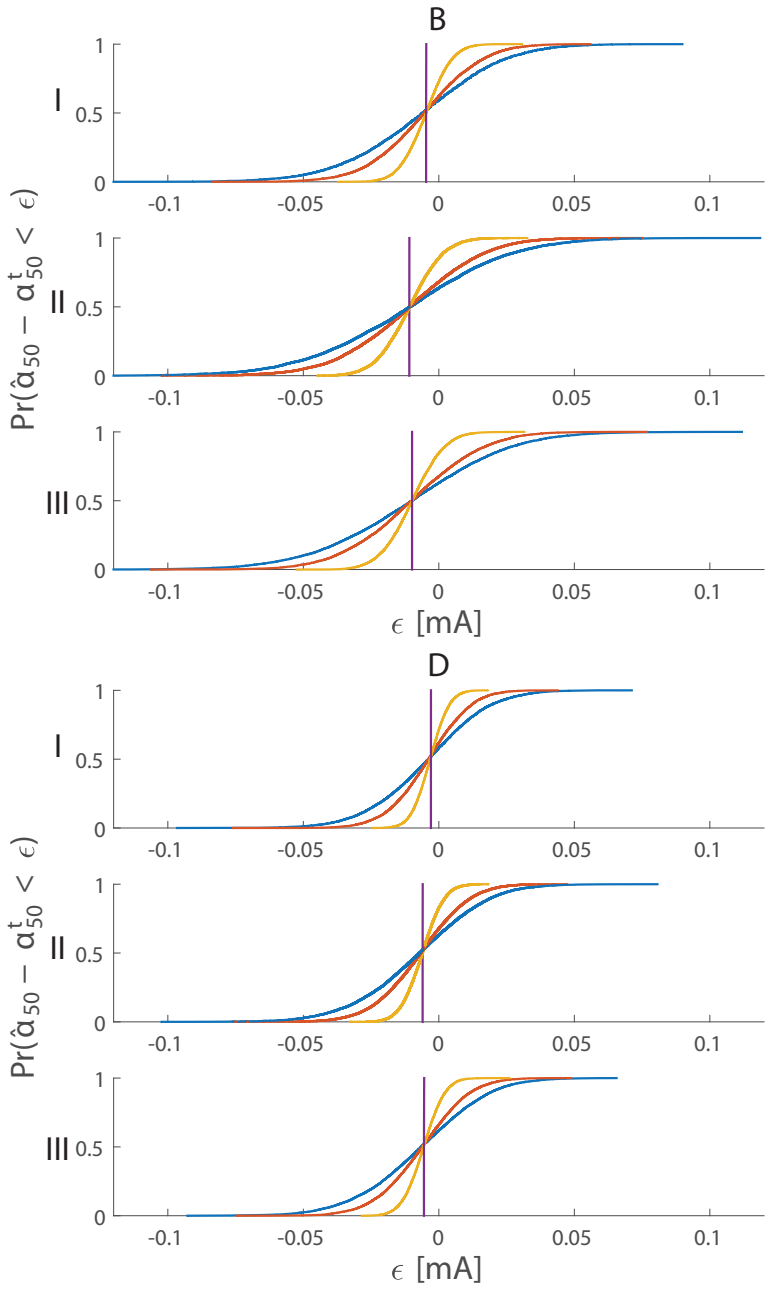

Figure 5.17: Estimation performance on detection thresholds for various cases with $\boldsymbol{\theta}^{(1)}$ and four combinations of three temporal properties listed in Table 5.2. Panel A-D correspond to combination A-D, respectively. Label I, II and III indicate the SSP, the APP with $N o A=5$, and the APP with $N o A=10$, respectively. 

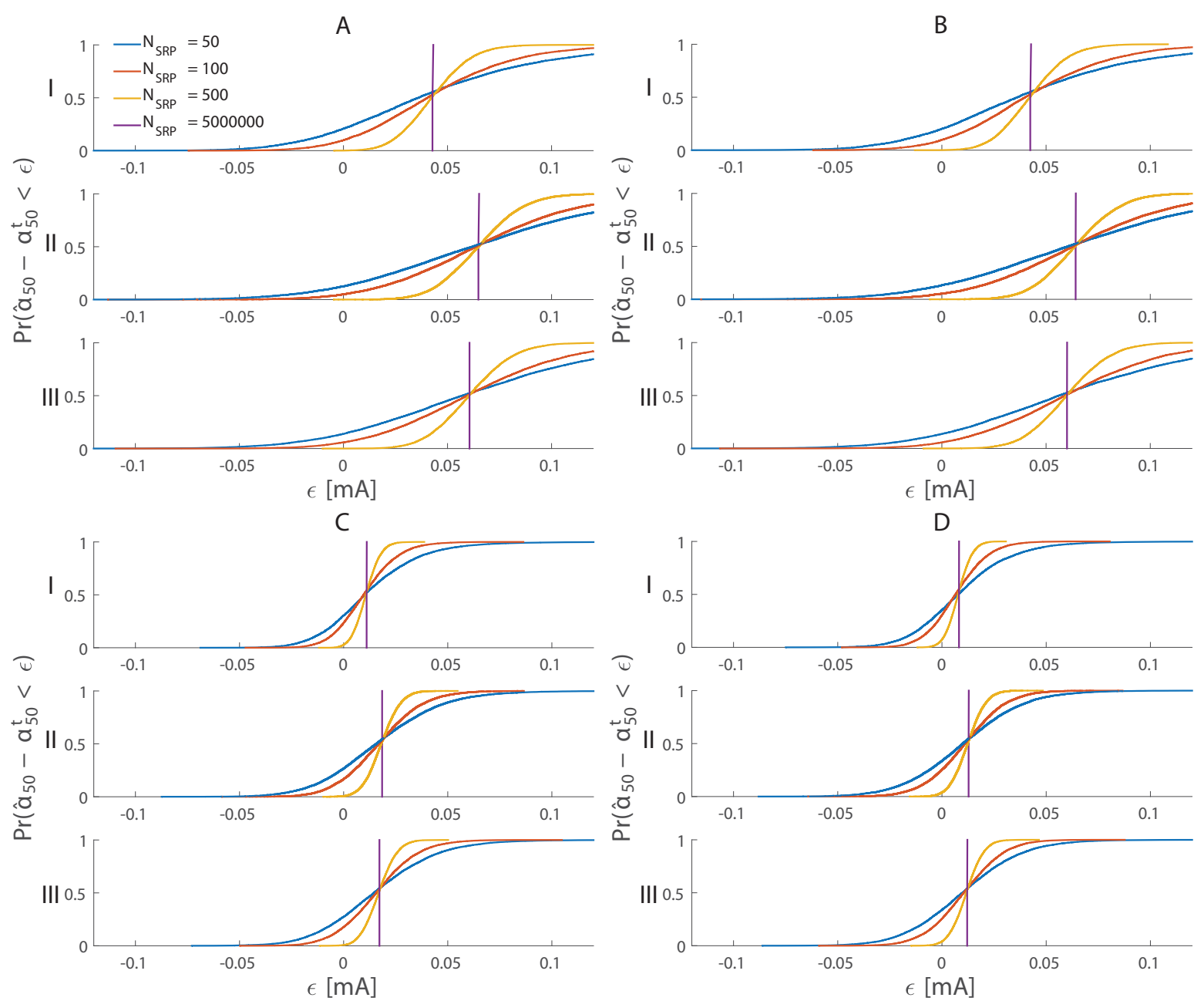

Figure 5.18: Estimation performance on detection thresholds for various cases with $\boldsymbol{\theta}^{(2)}$ and four combinations of three temporal properties listed in Table 5.2. Panel A-D correspond to combination A-D, respectively. Label I, II and III indicate the SSP, the APP with $N o A=5$, and the APP with $N o A=10$, respectively. 


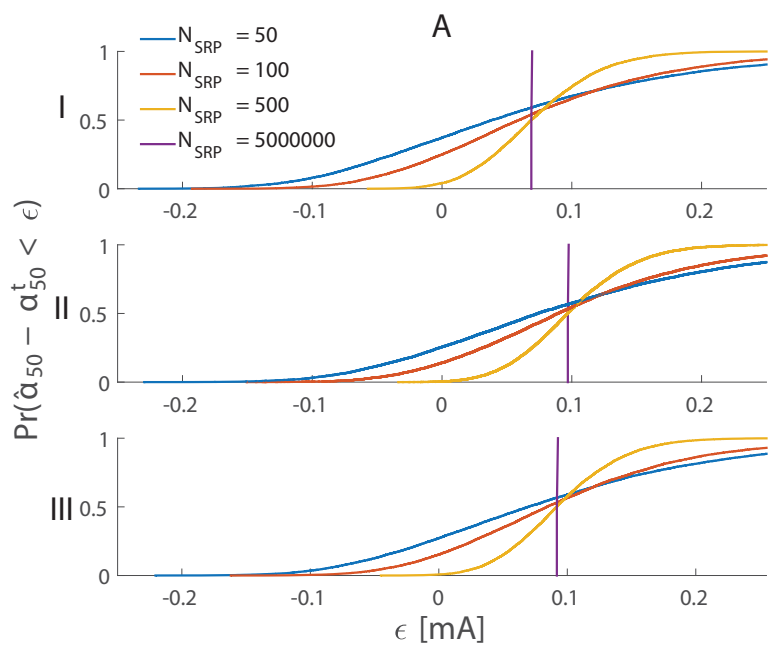

$\mathrm{C}$

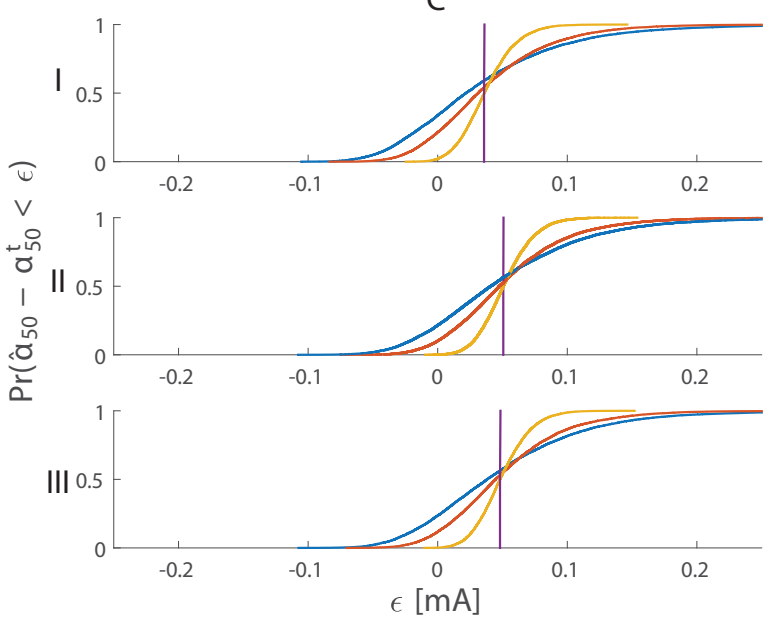

B

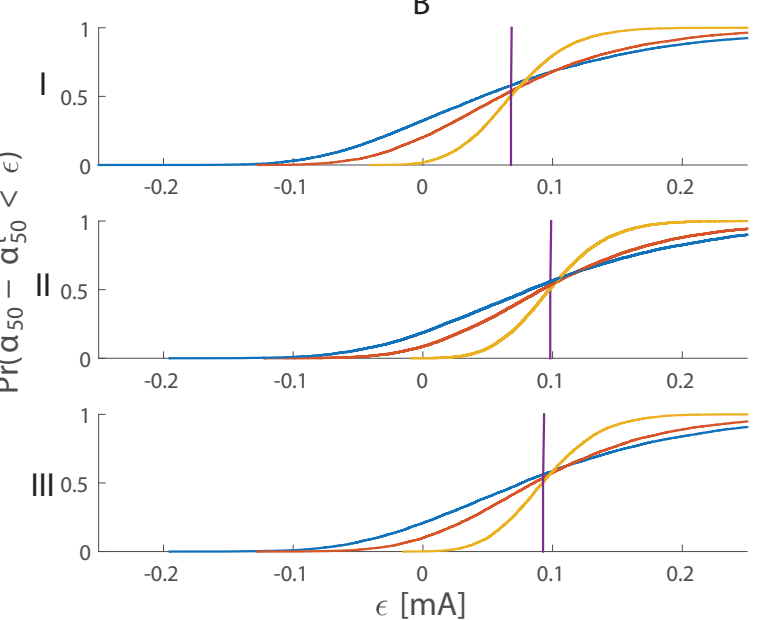

$\mathrm{D}$
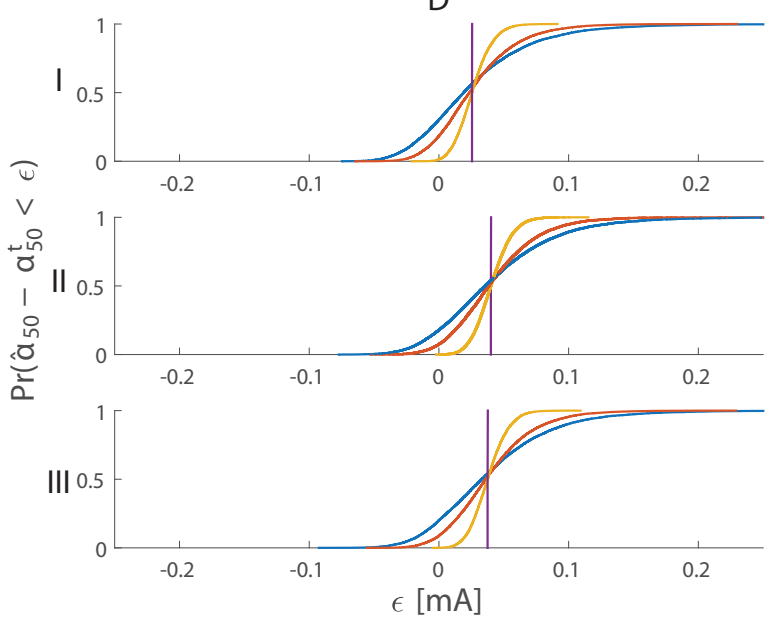

Figure 5.19: Estimation performance on detection thresholds for various cases with $\boldsymbol{\theta}^{(3)}$ and four combinations of three temporal properties listed in Table 5.2. Panel A-D correspond to combination A-D, respectively. Label I, II and III indicate the SSP, the APP with $N o A=5$, and the APP with $N o A=10$, respectively. 

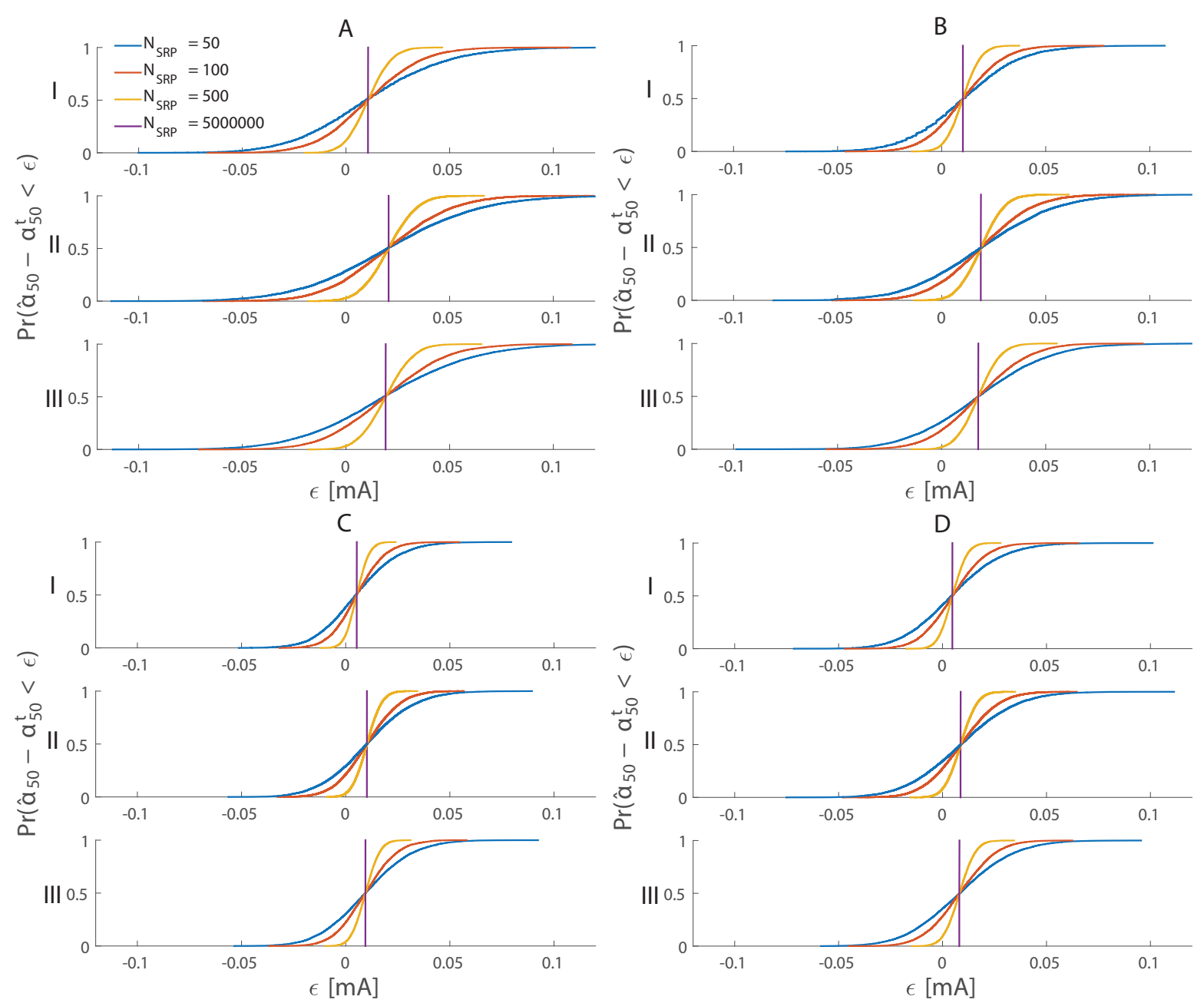

Figure 5.20: Estimation performance on detection thresholds for various cases with $\boldsymbol{\theta}^{(4)}$ and four combinations of three temporal properties listed in Table 5.2. Panel A-D correspond to combination A-D, respectively. Label I, II and III indicate the SSP, the APP with $N o A=5$, and the APP with $N o A=10$, respectively. 


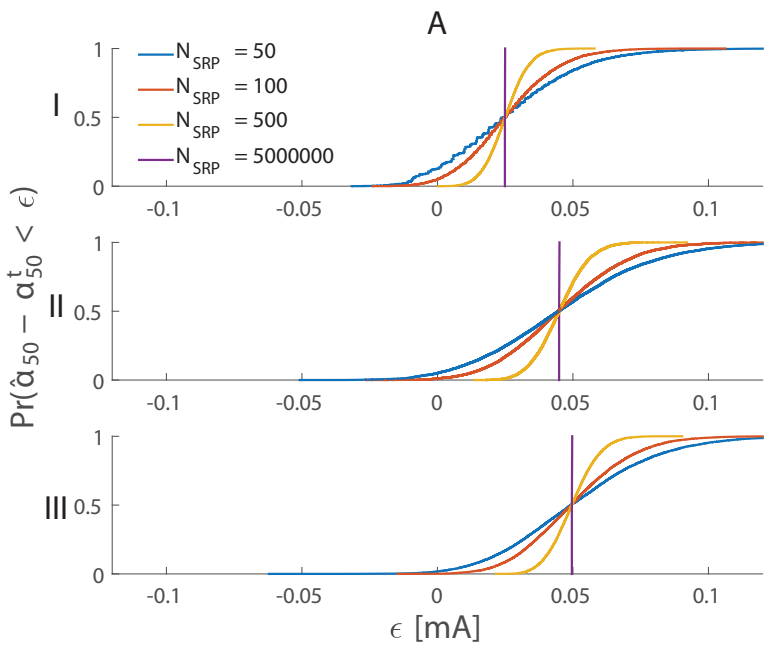

$\mathrm{C}$
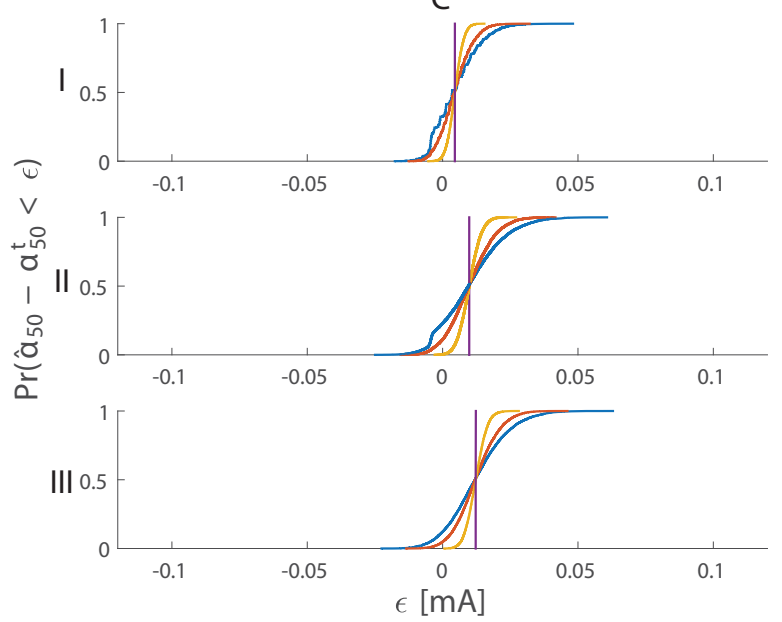

B

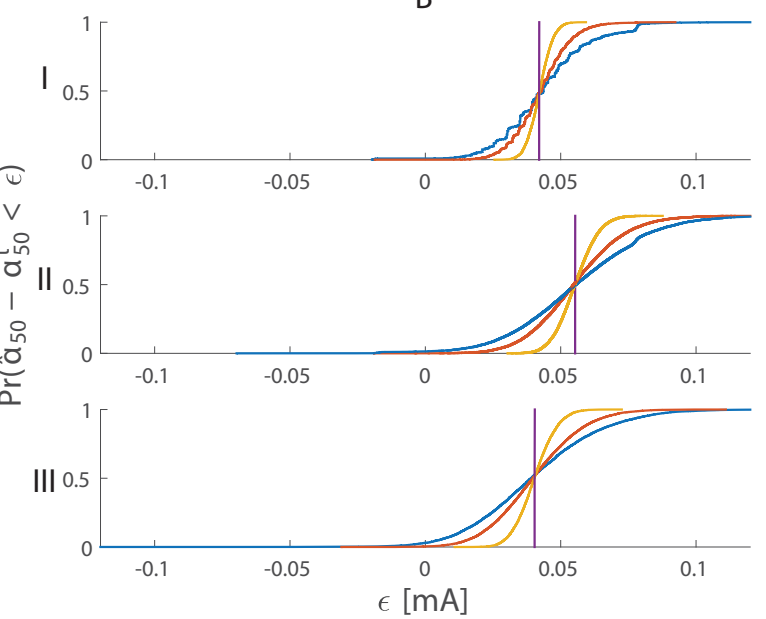

$\mathrm{D}$
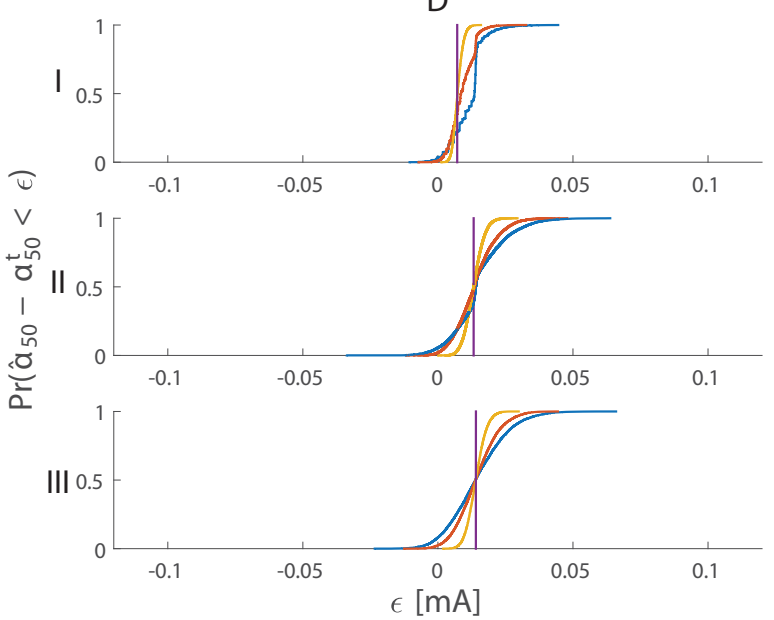

Figure 5.21: Estimation performance on detection thresholds for various cases with $\boldsymbol{\theta}^{(5)}$ and four combinations of three temporal properties listed in Table 5.2. Panel A-D correspond to combination A-D, respectively. Label I, II and III indicate the SSP, the APP with $N o A=5$, and the APP with $N o A=10$, respectively. 

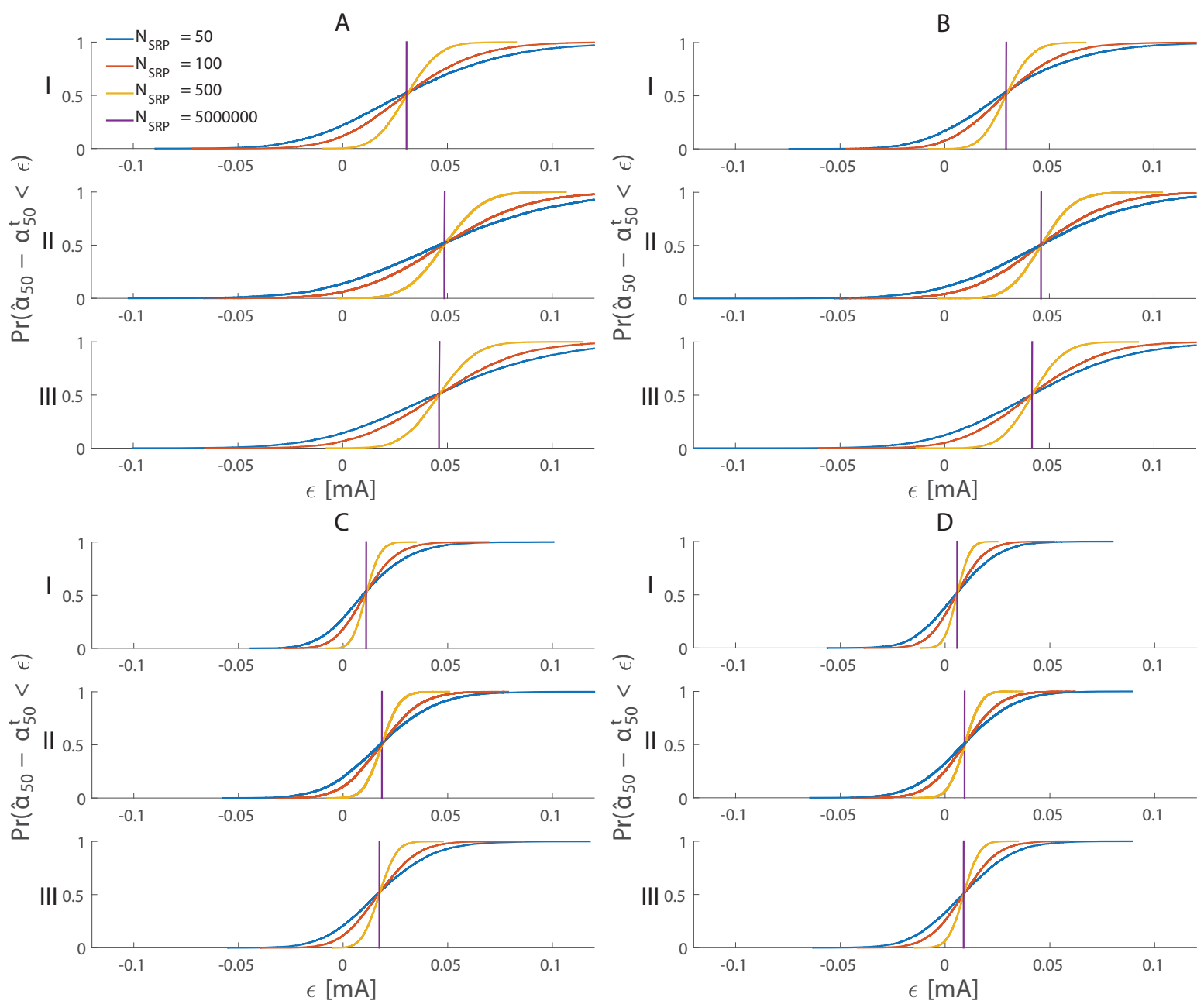

Figure 5.22: Estimation performance on detection thresholds for various cases with $\boldsymbol{\theta}^{(6)}$ and four combinations of three temporal properties listed in Table 5.2. Panel A-D correspond to combination A-D, respectively. Label I, II and III indicate the SSP, the APP with $N o A=5$, and the APP with $N o A=10$, respectively. 

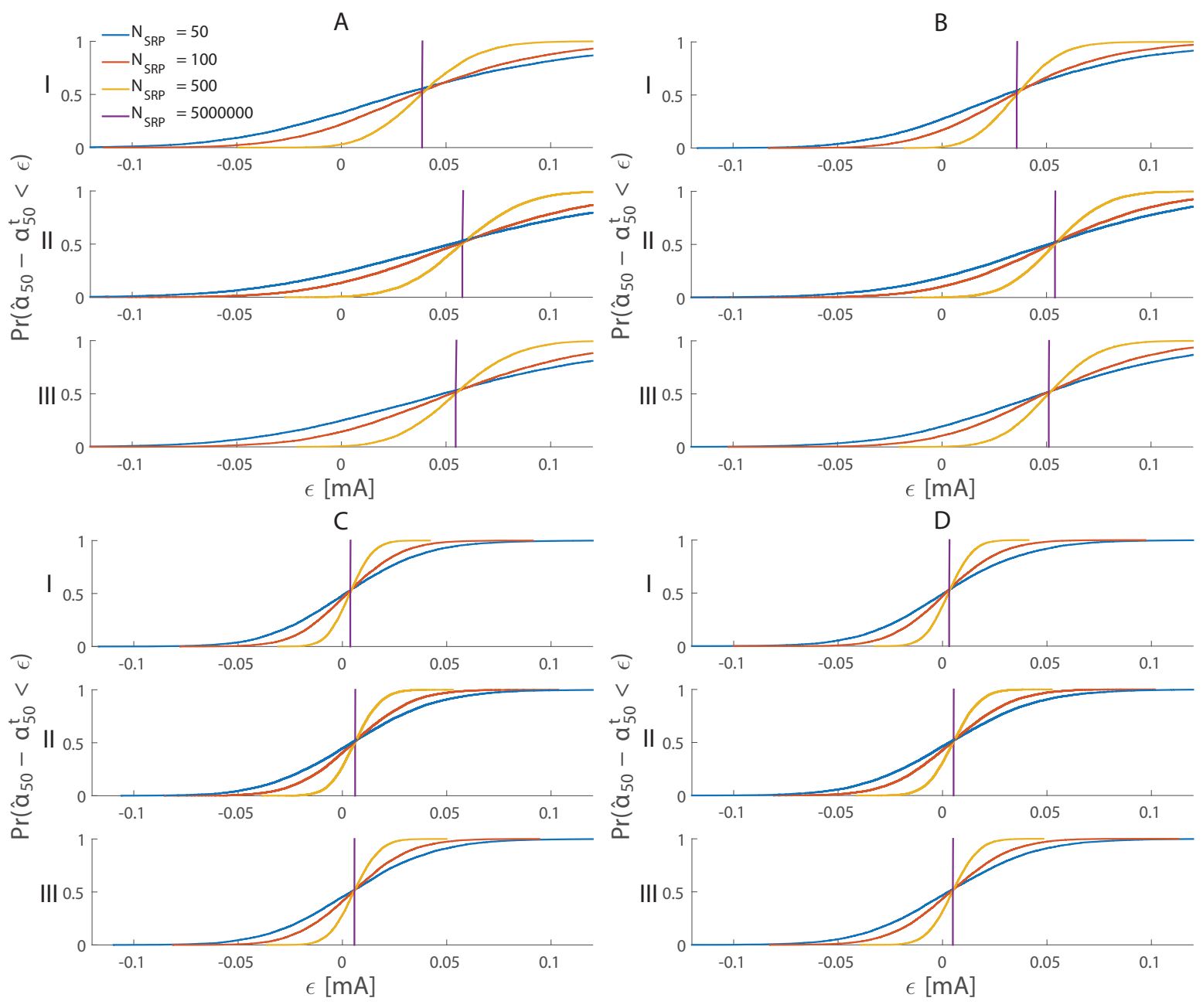

Figure 5.23: Estimation performance on detection thresholds for various cases with $\boldsymbol{\theta}^{(7)}$ and four combinations of three temporal properties listed in Table 5.2. Panel A-D correspond to combination A-D, respectively. Label I, II and III indicate the SSP, the APP with $N o A=5$, and the APP with $N o A=10$, respectively. 


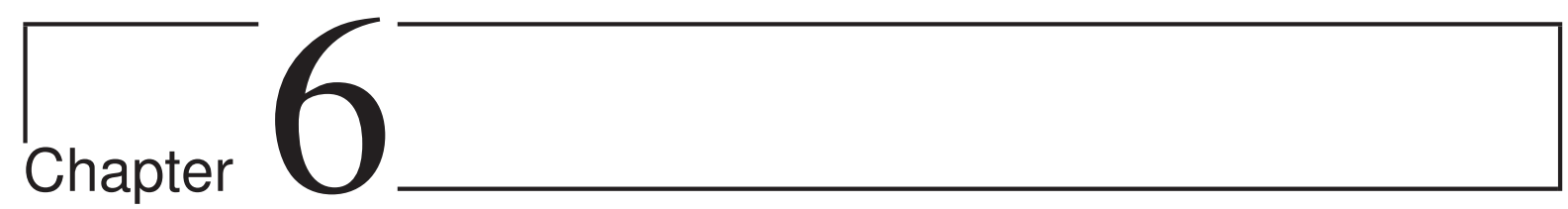

Discussion, Outlook and Concluding Remarks 


\section{Discussion and Outlook}

This thesis focuses on the development of computational approaches to understand essential nociceptive mechanisms. Psychophysical data from a detection task constrain and support predictions of developed computational models. In addition, some new directions could deserve further explorations. As general discussion of the thesis, we start with the relevant nociceptive mechanisms, where we also point out possible extensions of modeling of these mechanisms. Next, we discuss possible alternatives to analyze psychophysical data.

\section{Nociceptive mechanisms and possible model extensions}

Our developed models in Chapter 2 represent essential peripheral and central nociceptive subsystems. These models also account for both neural integration as well as the principle of probability summation. For possible extensions of the models to understand other relevant mechanisms, we start with the interface for peripheral activation. Electrocutaneous stimulation with a needle electrode preferentially activates free nerve endings of $\mathrm{A} \delta$ fibers. In the present modeling in Chapter 2, we assumed that the endings under the needle electrode terminated at the same depth in the epidermis. One can justify this approximation as the applied amplitudes are relatively low (less than $2 \mathrm{~mA}$ ). This type of stimulation leads to focal excitation of nerve endings (Inui et al., 2002; Mouraux et al., 2010), which diminishes the effect of the inhomogeneity on peripheral activation. As a result, we modeled the relation between peripheral activation and the applied amplitude as a threshold linearity, namely a dead zone followed by a linear response zone. However, if one would apply painful stimuli which require higher amplitudes, inhomogeneity of depths of endings should be incorporated for realistic modeling. If the endings locate at distinct depths but still lie in the epidermal layer, the resultant peripheral activation will exhibit a more complex relation with respect to the applied amplitude. The activation is still expected to retain a dead zone, whose range is determined by the depth of most superficial endings. When the amplitude is above the activation threshold to escape from the dead zone, activation will exhibit a nonlinear relation with respect to the applied amplitude. In addition to inhomogeneity of endings of $\mathrm{A} \delta$ fibers, painful stimuli can recruit non-nociceptive $\mathrm{A} \beta$ fibers (Steenbergen et al., 2012), which are located deeper within the skin. To assess the contributions of different types of 
fibers, detailed modeling of the interface could be useful. For this, Mørch et al. (2011) considered both geometries of fibers and different tissue layers by building and analyzing a finite element model for surface electrodes. Further work with their modeled interface may advance understanding of peripheral activation in response to electrocutaneous stimulation using an intra-epidermal needle electrode.

In the periphery, the recruited free nerve endings generate spikes. Such activities travel along the nociceptive fibers from these endings to the dorsal horn. Our modeling did not take this process into account explicitly, because myelinated A $\delta$ fibers can ensure relatively robust and rapid transmission. However, in some scenarios, transmission along fibers could become substantially different. Then, extensions are useful to make models more realistic. As a first example, for patients with demyelinating diseases, the failure of spike propagation affects the robustness of transmission. For a single fiber, one can model the transmitted state at the presynaptic terminal, i.e. success or failure, as a random variable following a binomial distribution. For a substantial number of fibers, one can effectively model the amount of successfully transmitted presynaptic spikes following a normal distribution. The developed models (Chapter 2) contain stochastic processes for the postsynaptic activity: in the DDM, it is filtered white noise, while in the HM, it is a time-varying Poisson process. Then, accounting for stochastically transmitted states, extended models would involve a mixture of multiple stochastic processes. Such a mixture can challenge us to derive an analytically tractable model. This may also restrict practical applications of time consuming computational approaches, e.g. parameter estimation and identifiability analysis (Chapter 4). Second, there exists a spread of action potentials arriving at presynaptic terminals propagated along various fibers. Due to rapid transmission through $\mathrm{A} \delta$ fibers, the spread of action potentials is expected to be relatively narrow compared with a relatively large time constant in the central nociceptive processing (Prescott and Koninck, 2002; Weng et al., 2006). Under normal conditions, it is reasonable not to take this spread into account in modeling of postsynaptic activity. However in more general situations, we acknowledge that the width of the spread depends on multiple factors including the distance from the stimulation site to the dorsal horn as well as the conduction velocity of fibers. For example, when the lower leg is stimulated, it will double the distance in comparison to upper arm stimulation. Therefore, it could become necessary to consider the enlarged spread into the model. Considering a large number of presynaptic spikes, the spread can be treated as the probability density distribution of the arriving time, which is a continuous random variable. By choosing an 
appropriate distribution, the accordingly extended model could still be analytically tractable. Similarly, to represent C-fiber-mediated nociceptive function, a realistic model is expected to incorporate the above transmission processes.

To represent the central nociceptive subsystem, each of two developed models consisted of a deterministic process and a stochastic process, see Chapter 2. Regarding validity and possible extensions of these models, let us have a look at relevant neurophysiological processes. In a comprehensive view, the central nociceptive system consists of spinal dorsal horn neurons as well as third-order neurons, e.g. in the thalamus (Purves, 2008). It seems that the modeled deterministic part is an oversimplification of the entire central nociceptive system. First of all, we did not explicitly model supraspinal neuronal circuits. This is partially due to the lack of knowledge on neurometrics, i.e. how spikes generated by neurons are translated into humans' sensation or perception (Stüttgen et al., 2011). In this thesis, we assumed that a single spike represented sufficient activation in the central subsystem for a detected response. Second, in the spinal dorsal horn, the deterministic part represents the processing of monosynaptic projection neurons. We acknowledge that there are polysynaptic pathways in the spinal nociceptive system. These pathways often include a variety of interneurons in lamina II (Moore et al., 2000; Todd, 2010). However, in this thesis, we considered the nociceptive detection task rather than more widely implemented experimental paradigms using painful stimuli (Olesen et al., 2012). The detection task with low-intensity stimuli could result in preferential recruitments of monosynaptic projection neurons. In addition to heterogeneous spinal pathways, short-term synaptic plasticity would also be present upon application of pulse-train stimulation (Luo et al., 2014). However, it is still unknown whether the net outcome of the overall plasticity in the dorsal horn circuitry is excitatory or inhibitory. With a clearer characterization of the overall effect, we have indicated possible model extensions to represent short-term synaptic plasticity in Chapter 2. Those extensions will still keep the hazard model analytically tractable. Hence, a similar computational cost is expected for estimation and assessment of identifiability of parameters as that in Chapter 4. Third, both the drift-diffusion model and the hazard model could also be interpreted as accumulator models for binary detection responses, see (Bogacz et al., 2006; Ranger and Kuhn, 2014). From this top-down point of view, the modeled first-order system could be considered to capture the essential dynamics for the overall system including both spinal and supraspinal processes. 
In this thesis, we have checked the validity of our modeling work from a datadriven perspective. Chapter 2 and 3 showed qualitative agreement between model simulations and experimental observations of detection thresholds on a group level. Furthermore, Chapter 4 demonstrated reasonable goodness of fit of the hazard model to various datasets containing stimulus-response pairs measured from different individuals. These three chapters have already demonstrated the validity of these minimalistic models. In view of short-term synaptic plasticity, one prospective work could extend the model as discussed in Chapter 2. Then, one could evaluate the values of Bayesian information criterion for the extended model with the original one. Comparison of the results could answer the question whether short-term synaptic plasticity is effectively present in the nociceptive system.

Our work assumed that the nociceptive function was stationary, namely the psychometric function was invariant during the 10-minute experimental section. However, time scales of nociceptive modulations are in the order of tens of minutes, like conditioned pain modulation (CPM) (Yarnitsky et al., 2010; Lewis et al., 2012a). The clinical relevance to assess CPM is recently recognized, as an impaired CPM is often present in patients with chronic pain (Lewis et al., 2012b). Current measurement techniques mainly rely on QSTs using painful stimuli. As experimental work of the PAINSIGHT project, Doll et al. (2014) reported a human subject study with application of a cold pressor test (CPT). In this experiment, human subjects performed a detection task during and after the CPT. Consequently, the induced conditioned pain modulation might influence the stationarity of the psychometric function. The current biological interpretation of the nociceptive modulation is attributed to supraspinal neuronal dynamics (Millan, 2002). However, it is unknown to what extent CPT affects human nociceptive transmission. In addition, this experiment (Doll et al., 2014) could evoke cognitive processes like learning or fatigue, which could also modulate the nociceptive transmission. Hence, to separate and characterize distinct types of modulations is challenging. In future, to account for the CPM induced by a CPT, one may extend the present hazard model by incorporating relevant supraspinal dynamics. Also, our methods of parameter estimation and identifiability analysis may be adapted to increase quantitative understanding of the nociceptive modulation. 


\section{Clinical application}

Experimental work in the PAINSIGHT project has proposed novel observation techniques for the overall nociceptive state by using a simple detection task (Doll et al., 2014, 2015a,c). For clinical implications, this thesis initiated computational modeling and analysis to link nociceptive mechanisms to observations from the nociceptive detection task. First, the computational approach developed in this thesis can be viewed as a valuable tool in generating new hypotheses about nociceptive processing. For example, based on psychophysical measurements (Doll et al., 2015c), our modeling study implied distinct sensitization processes upon application of a high-dose capsaicin patch (Chapter 3). In this experimental protocol, no other medical interventions to prevent sensitization were applied. In further studies, one may design new experimental protocols to validate existence of those suggested sensitization processes. Application of medication such as ketamine which is considered to prevent central sensitization (Woolf, 2011) would help to check the validity of our present study. By collecting and analyzing detection thresholds, one may compare observed patterns of changes of thresholds over time to our model predictions. Therefore, one could test the hypothesis about central sensitization, which was suspected to last over one month. In turn, this may help to explore more efficient drugs to prevent sensitization to achieve better pain relief for patients with pain disorders. In other case, future model refinements would also benefit from disagreement between observations and model predictions.

Second, our model-based approaches could enable clinicians to perform mechanismbased diagnosis to differentiate malfunctioning processes in the peripheral and central nociceptive subsystems of patients. Our work has demonstrated that model parameters can be physiologically meaningful (Chapter 2 and 3) and identifiable (Chapter 4). Here, for a differential diagnosis, we revisit meanings of these six lumped parameters retained in the HM about whether they meet the essential requirement. Two parameters $\lambda_{L}$ and $\tau_{2}$ represent pure central properties of the nociceptive system, namely, the maximal firing frequency and time constant of secondary neurons, respectively. In contrast, $\alpha_{1}$ and $\tau_{1}$ characterize the peripheral nociceptive subsystem. Two remaining parameters $\alpha_{L}$ and $\sigma_{L}$ represent compound properties of both peripheral and central subsystems. However, an appropriate reparametrization of these two parameters, e.g. $\frac{\alpha_{L}}{\sigma_{L}}$ (equivalent to $\frac{\alpha_{h}}{\sigma_{h}}$ ), could retain some pure central property of the nociceptive system. In the models, the fiber density $\rho$ and the synaptic conductance $\bar{g}$ are always present as a product form in the lumped parame- 
ters $\alpha_{L}$ and $\sigma_{L}$. This implies the structural non-identifiability of those two physical quantities. To resolve this non-identifiability, more efforts are required in a design of new observation techniques and modeling. For future applications, let us look at recently developed measurement techniques and suspected malfunctioning mechanisms in patients with specific pain disorders. Abnormal nociceptive mechanisms were recently studied with various quantitative sensory testing, e.g. central sensitization for osteoarthritis see (Lluch et al., 2014). In contrast to the detection task considered in this thesis, this study utilized QST with painful stimuli. So far, the nociceptive detection task has been implemented in psychophysical experiments for healthy subjects, including those who underwent capsaicin treatments. Translation of gained insights from such an experimental setting into a clinical setting requires further clinical studies that involve potential patients with pain disorders. For that, a clinical study on a specific group of patients (e.g. with total knee arthroplasty), would check the applicability of the nociceptive detection task. With these measurements, to differentiate distinct malfunctioning processes, clinical applicability of our computational tools might be validated and tailored by considering patients' data. By applying parameter estimation, the alterations of suspected central nociceptive mechanisms might be reflected in changes of estimates of these parameters. Clinicians may utilize such model-based interpretations into improved mechanism-based treatments for chronic pain patients.

\section{Developments of computational approaches}

As the primary aim of this thesis is to increase our understanding of nociceptive mechanisms, we developed parameter estimation and identifiability tools. Due to the data-driven nature of these computational statistics, we discuss further developments of these approaches regarding relevant psychophysical measurements.

\section{Psychophysical measurements}

In this thesis, we considered two psychophysical outcomes from the detection task, namely detection thresholds and stimulus-response pairs. Modeling work in Chapter 2 and 3 achieved qualitative agreement to detection thresholds from a group of healthy subjects. Chapter 4 integrated the hazard model directly with individual's stimulus-response pairs to estimate six lumped model parameters. We did not perform estimation to determine model parameters from intermediate estimates 
of detection thresholds, as logistic regression may introduce a bias in the estimate of the detection threshold, see Chapter 5.

Because the drift-diffusion model in general is analytically intractable, parameter estimation is hampered and was not implemented in this thesis. In a detection task, reaction times are often measured for the detected responses (Kingdom and Prins, 2010). This additional type of measurements would provide extra information about the underlying processes. For measured reaction times, a suitable estimation approach needs to be developed. As extensions of the conventional logistic regression, (Palmer et al., 2005; Stone, 2014) proposed approaches to estimate parameters with measurements of reaction times. Regarding the computational models in Chapter 2 , we discuss an extension involving reaction times. For the pure DDM without a leaky term, analytical arguments on reaction times can be straightforwardly determined (Bogacz et al., 2006). For a DDM with a leaky term, Roy and Smith (1969) derived analytical solutions for the mean of the first passage time. Recently, Srivastava et al. (2015) initiated a framework to evaluate the probability density function of the first passage time from variants of the DDM. The integration of the reaction times with the DDM may facilitate the parameter estimation for model parameters, see (Bogacz et al., 2006). For the hazard model with a Poisson process, the model can generate the probability density function of reaction times in a straightforward way (Plesser and Gerstner, 2000). For our hazard model, one can compute its counterpart in a similar and efficient way. Possible integration with reaction time may help to improve estimation of system parameters in the HM.

\section{Frequentist or Bayesian}

Computational approaches to estimate model parameters can be divided into the frequentist and Bayesian schools (Raue et al., 2013). The most used frequentist methods are based on either bootstrap techniques (Alcalá-Quintana and GarcíaPérez, 2013) or the profile likelihood (Raue et al., 2009). In this thesis, we adapted the PL approach to assess the parameter identifiability of parameters of the HM. For a bootstrap-based approach, Alcalá-Quintana and García-Pérez (2013) applied bootstrap techniques to assess the identifiability of a physiology-based model representing mechanisms for time perception. One can easily implement the bootstrap-based approach in our setting. However, the possible existence of structural non-identifiability shown in Chapter 4 could hamper the applicability in assessing parameter identifiability (Fröhlich et al., 2014). In contrast to frequentist methods, Bayesian methods 
estimate parameters by evaluating the posterior distribution of model parameters, where Markov Chain Monte Carlo (MCMC) is the workhorse (Vanlier et al., 2013). Obtained posterior distribution is useful in assessment of uncertainty of parameter estimates. Moreover, posterior distribution of parameters can be used to assess uncertainty of model predictions (van Mourik et al., 2014). This does not require a heavy computational burden like nonlinear optimizations involved in the profile likelihood approach. Regarding the applicability of these approaches, comparison studies have been performed within systems biology (Raue et al., 2013; Fröhlich et al., 2014). Those studies remarked that in case of structural non-identifiability, the profile likelihood approach is still applicable whereas the MCMC failed. As our work has already found some non-identifiable cases for the HM, it would be best to first resolve the non-identifiability of model parameters, and thereafter to perform other techniques such as the MCMC in a Bayesian framework to assess uncertainty in both model parameters and predictions.

\section{Group-level or Individual-level}

We developed and calibrated computational models of the nociceptive system using psychophysical data from individuals. In our identifiability analysis in Chapter 4 using datasets from 15 subjects, we found that six model parameters were sometimes identifiable. The non-identifiability could be attributed to a limited amount of information contained in each individual's dataset. To resolve that, we proposed and performed a model-based approach to select informative stimulus properties for further measurements on the same subject. As an alternative method to identify model parameters, one could perform a group-level analysis, i.e. using datasets from a group of subjects. To model psychophysical measurements, generalized linear mixed effect model (GLMM) was used to replicate data collected from a group of subjects (Knoblauch and Maloney, 2012; Moscatelli et al., 2012). GLMMs contain fixed and random effects to represent the group average and the inter-individual variability, respectively. As these models are linearizable via a link function, e.g. a logistic function, they are identifiable in general. However, nonlinear models often challenge estimation procedures, which may lead to non-identifiability of estimates. To model psychophysical data, there are few studies using a nonlinear mixed effect model (NLMM), see e.g. (Cheung et al., 2008). However, NLMMs have been extensively addressed in the field of population Pharmacokinetic/Pharmacodynamic (PK/PD) modeling. This modeling approach integrates pharmacokinetic and phar- 
macodynamic data from a group of subjects in response to some drug. In pain research, PK/PD modeling techniques have been applied (Danhof et al., 2008; Martini et al., 2011). Some developed tools like the NONMEM (Bauer et al., 2007) may be used or adapted in future for variants of the developed HM as a group-level modeling approach.

In conventional mixed modeling studies, the random effect, i.e. inter-individual variability, is represented by a unimodal normal distribution (Yang et al., 2014). Physiological processes could be subject to more variability, resulting in some subgroup feature, e.g. due to gender or genetic difference. At a group level, the assumption with a unimodal distribution for the random effect could be questionable. To account for multiple modes, a mixture of the distribution was proposed and relevant modeling techniques were developed, e.g. (Böhning et al., 2007; Komárek and Lesaffre, 2008). NLMMs with mixture features might reveal subgroups of subjects with distinct characteristics in response to medical interventions. To investigate pharmacodynamic characteristics upon application of a high-dose capsaicin patch for pain-suffering patients, Martini et al. (2012) built a NLMM with such a mixture feature to study how subjects responded to capsaicin patch treatment. Their results indicated four subgroups of subjects with distinct responses. A prospective modeling study might integrate measured stimulus-response pairs before and after capsaicin patch treatment (Doll et al., 2015c). Based on the HM, mixture features could be incorporated into some lumped parameters to account for potential subgroups. Hence, further adaptions of above group-level modeling techniques with psychophysical data could increase our understanding of underlying physiological and cognitive processes.

\section{Concluding Remarks}

This thesis provides a foundation of computational tools to understand essential nociceptive mechanisms underlying a detection task. Our studies demonstrated the applicability of computational modeling and analysis. Further developments of these computational approaches may advance our insights into human nociceptive processing. In addition, these approaches might be valuable towards a differential diagnosis and improved therapeutics for pain disorders. 


\section{References}

Akopian, A.N., Abson, N.C., and Wood, J.N. (1996). Molecular genetic approaches to nociceptor development and function. Trends in neurosciences, 19(6):240-246.

Alcalá-Quintana, R. and García-Pérez, M.A. (2013). Fitting model-based psychometric functions to simultaneity and temporal-order judgment data: MATLAB and R routines. Behavior research methods, 45(4):972-998.

Anand, P. and Bley, K. (2011). Topical capsaicin for pain management: therapeutic potential and mechanisms of action of the new high-concentration capsaicin $8 \%$ patch. British journal of anaesthesia, 107(4):490-502.

Arendt-Nielsen, L. and Curatolo, M. (2013). Mechanistic, translational, quantitative pain assessment tools in profiling of pain patients and for development of new analgesic compounds. Scandinavian Journal of Pain, 4(4):226-230.

Argüello, E.J., Silva, R.J., Huerta, M.K., and Avila, R.S. (2015). Computational modeling of peripheral pain: a commentary. Biomedical engineering online, 14(1):56.

Baron, R. (2006). Mechanisms of disease: neuropathic pain-a clinical perspective. Nature Clinical Practice Neurology, 2(2):95-106.

Bauer, R.J., Guzy, S., and Ng, C. (2007). A survey of population analysis methods and software for complex pharmacokinetic and pharmacodynamic models with examples. The AAPS journal, 9(1):E60-E83.

Bazanella, A.S., Bombois, X., and Gevers, M. (2012). Necessary and sufficient conditions for uniqueness of the minimum in prediction error identification. Automatica, 48(8):1621-1630.

Bellman, R. and Åström, K.J. (1970). On structural identifiability. Mathematical biosciences, $7(3): 329-339$.

Bellu, G., Saccomani, M.P., Audoly, S., and D'Angiò, L. (2007). Daisy: A new software tool to test global identifiability of biological and physiological systems. Computer Methods and Programs in Biomedicine, 88(1):52-61.

Billings, S.A. (1980). Identification of nonlinear systems-a survey. Control Theory and Applications, 
IEE Proceedings D, 127(6):272-285.

Binshtok, A.M., Bean, B.P., and Woolf, C.J. (2007). Inhibition of nociceptors by TRPV1-mediated entry of impermeant sodium channel blockers. Nature, 449(7162):607-610.

Blumenthal, T.D., Avendano, A., and Berg, W.K. (1987). The startle response and auditory temporal summation in neonates. Journal of Experimental Child Psychology, 44(1):64-79.

Bogacz, R., Brown, E., Moehlis, J., Holmes, P., and Cohen, J.D. (2006). The physics of optimal decision making: a formal analysis of models of performance in two-alternative forced-choice tasks. Psychological review, 113(4):700.

Böhning, D., Seidel, W., Alfó, M., Garel, B., Patilea, V., and Walther, G. (2007). Advances in mixture models. Computational Statistics \& Data Analysis, 51(11):5205-5210.

Britton, N.F., Chaplain, M.A.J., and Skevington, S.M. (1996). The role of n-methyl-d-aspartate (NMDA) receptors in wind-up: A mathematical model. Mathematical Medicine and Biology, 13(3):193-205.

Britton, N.F. and Skevington, S.M. (1989). A mathematical model of the gate control theory of pain. Journal of theoretical biology, 137(1):91-105.

Capocelli, R.M. and Ricciardi, L.M. (1971). Diffusion approximation and first passage time problem for a model neuron. Kybernetik, 8(6):214-223.

Carr, F.B. and Zachariou, V. (2014). Nociception and pain: lessons from optogenetics. Frontiers in behavioral neuroscience, 8.

Cheung, S-H., Kallie, C.S.., Legge, G.E., and Cheong, A.M.Y. (2008). Nonlinear mixed-effects modeling of MNREAD data. Investigative ophthalmology $\&$ visual science, 49(2):828.

Chiş, O.-T., Banga, J.R., and Balsa-Canto, E. (2011). Structural identifiability of systems biology models: A critical comparison of methods. PLoS ONE, 6(11):e27755.

Clopper, C.J. and Pearson, E.S. (1934). The use of confidence or fiducial limits illustrated in the case of the binomial. Biometrika, 26(4):404-413.

Coleman, T.F. and Li, Y. (1996). An interior trust region approach for nonlinear minimization subject to bounds. SIAM Journal on optimization, 6(2):418-445.

Constantin, P. and Foias, C. (1988). Navier-stokes equations. University of Chicago Press.

Coombes, S., Thul, R., Laudanski, J., Palmer, A.R., and Sumner, C.J. (2011). Neuronal spike-train responses in the presence of threshold noise. Frontiers in life science, 5(3-4):91-105.

Coutaux, A., Adam, F., Willer, J.-C., and Le Bars, D. (2005). Hyperalgesia and allodynia: peripheral mechanisms. Joint Bone Spine, 72(5):359-371.

Cruz-Almeida, Y. and Fillingim, R.B. (2014). Can quantitative sensory testing move us closer to mechanism-based pain management? Pain Medicine, 15(1):61-72.

Czepita, D. (2002). Myopia-epidemiology, pathogenesis, present and coming possibilities of treatment. American Journal of Case Reports, 3(4):294-300.

Danhof, M., de Lange, E.C.M., Della Pasqua, O.E., Ploeger, B.A., and Voskuyl, R.A. (2008). Mechanism-based pharmacokinetic-pharmacodynamic (pk-pd) modeling in translational drug research. Trends in pharmacological sciences, 29(4):186-191. 
Derman, C. (1957). Non-parametric up-and-down experimentation. The Annals of Mathematical Statistics, 28(3):795-798.

Di Nardo, E., Nobile, A.G., Pirozzi, E., Ricciardi, L.M., and Rinaldi, S. (2000). Simulation of gaussian processes and first passage time densities evaluation. In Computer Aided Systems Theory-EUROCAST'99, pages 319-333. Springer.

Dixon, W. and Mood, A. (1948). A method for obtaining and analyzing sensitivity data. Journal of the American Statistical Association, 43(241):109-126.

Doll, R.J., Buitenweg, J.R., Meijer, H.G.E., and Veltink, P.H. (2014). Tracking of nociceptive thresholds using adaptive psychophysical methods. Behavior Research Methods, 46(1):55-66.

Doll, R.J., Maten, A.C.A., Spaan, P.G., Veltink, P.H., and Buitenweg, J.R. (2015a). Effect of temporal stimulus properties on the nociceptive detection probability using intra-epidermal electrical stimulation. Experimental Brain Research: accepted.

Doll, R.J., Veltink, P.H., and Buitenweg, J.R. (2015b). Observation of time-dependent psychophysical functions and accounting for threshold drifts. Attention, Perception, ES Psychophysics, $77(4): 1440-1447$.

Doll, R.J. and others (2015c). Responsiveness of electrical nociceptive detection thresholds to capsaicin $(8 \%)$ induced changes in nociceptive processing. In preparation.

Durrett, R. (2012). Essentials of stochastic processes. Springer Science \& Business Media.

Dworkin, R.H., Backonja, M., Rowbotham, M.C., Allen, R.R., Argoff, C.R., Bennett, G.J., Bushnell, M.C., Farrar, J.T., Galer, B.S., Haythornthwaite, J.A., et al. (2003). Advances in neuropathic pain: diagnosis, mechanisms, and treatment recommendations. Archives of neurology, 60(11):1524-1534.

Ehrenstein, W.H. and Ehrenstein, A. (1999). Psychophysical methods. In Modern techniques in neuroscience research, pages 1211-1241. Springer.

Faisal, A.A., Selen, L.P.J., and Wolpert, D.M. (2008). Noise in the nervous system. Nature Reviews Neuroscience, 9(4):292-303.

Farajidavar, A., Saeb, S., and Behbehani, K. (2008). Incorporating synaptic time-dependent plasticity and dynamic synapse into a computational model of wind-up. Neural Networks, 21(2):241249 .

FDA Center for Drug Evaluation and Research (2009). Medical review: Qutenza. http://www . accessdata.fda.gov/drugsatfda_docs/nda/2009/022395s000Medr.pdf. Accessed: 2010-09-30.

Fechner, G.T. (1860). Elemente der Psychophysik. Breitkopf \& Härtel.

Fors, U., Ahlquist, M.L., Skagerwall, R., Edwall, L.G.A., and Haegerstam, G.A.T. (1984). Relation between intradental nerve activity and estimated pain in man - a mathematical model. Pain, 18(4):397-408.

Friston, K.J. (1994). Functional and effective connectivity in neuroimaging: a synthesis. Human brain mapping, 2(1-2):56-78.

Fröhlich, F., Theis, F.J., and Hasenauer, J. (2014). Uncertainty analysis for non-identifiable dynamical systems: Profile likelihoods, bootstrapping and more. In Computational Methods in 
Systems Biology, pages 61-72. Springer.

Gabbiani, F., Midtgaard, J., and Knopfel, T. (1994). Synaptic integration in a model of cerebellar granule cells. Journal of neurophysiology, 72(2):999-1009.

García-Pérez, M.A. and Alcalá-Quintana, R. (2005). Sampling plans for fitting the psychometric function. The Spanish journal of psychology, 8(2):256-289.

Gargash, B.C. and Mital, D.P. (1980). A necessary and sufficient condition of global structural identifiability of compartmental models. Computers in Biology and Medicine, 10(4):237-242.

Gescheider, G.A., Berryhill, M.E., Verrillo, R.T., and Bolanowski, S.J. (1999). Vibrotactile temporal summation: probability summation or neural integration? Somatosensory 83 motor research, 16(3):229-242.

Gilchrist, J. and Bosmans, F. (2012). Animal toxins can alter the function of Nav1. 8 and Nav1. 9. Toxins, 4(8):620-632.

Gold, J.I. and Ding, L. (2013). How mechanisms of perceptual decision-making affect the psychometric function. Progress in neurobiology, 103:98-114.

Goldstein, E. (2009). Sensation and perception. Cengage Learning.

Granot, M., Sprecher, E., and Yarnitsky, D. (2003). Psychophysics of phasic and tonic heat pain stimuli by quantitative sensory testing in healthy subjects. European Journal of Pain, 7(2):139143.

Granstein, R.D. and Luger, T.A. (2009). Neuroimmunology of the Skin. Springer.

Haefeli, J., Kramer, J.L.K., Blum, J., and Curt, A. (2014). Heterotopic and homotopic nociceptive conditioning stimulation: Distinct effects of pain modulation. European Journal of Pain, 18(8):1112-1119.

Hamill, O.P., Marty, A., Neher, E., Sakmann, B., and Sigworth, F.J. (1981). Improved patchclamp techniques for high-resolution current recording from cells and cell-free membrane patches. Pflügers Archiv, 391(2):85-100.

Hodgkin, A.L. and Huxley, A.F. (1952). A quantitative description of membrane current and its application to conduction and excitation in nerve. The Journal of physiology, 117(4):500-544.

Hunter, I.W. and Korenberg, M.J. (1986). The identification of nonlinear biological systems: Wiener and hammerstein cascade models. Biological cybernetics, 55(2-3):135-144.

Inui, K., Tran, T.D., Hoshiyama, M., and Kakigi, R. (2002). Preferential stimulation of a $\delta$ fibers by intra-epidermal needle electrode in humans. Pain, 96(3):247-252.

Irnich, W. (2010). The terms "chronaxi" and "rheobase" are 100 years old. Pacing and clinical electrophysiology, 33(4):491-496.

Ji, R.R., Kohno, T., Moore, K.A., and Woolf, C.J. (2003). Central sensitization and LTP: do pain and memory share similar mechanisms? Trends in neurosciences, 26(12):696-705.

Kass, R.E. and Raftery, A.E. (1995). Bayes factors. Journal of the American Statistical Association, 90(430):773-795.

Kawasaki, Y., Kohno, T., Zhuang, Z.-Y., Brenner, G.J., Wang, H., Van Der Meer, C., Befort, K., Woolf, C.J., and Ji, R.-R. (2004). Ionotropic and metabotropic receptors, protein kinase a, 
protein kinase c, and src contribute to C-fiber-induced erk activation and camp response elementbinding protein phosphorylation in dorsal horn neurons, leading to central sensitization. The Journal of neuroscience, 24(38):8310-8321.

Kearney, R.E. and Hunter, I.W. (1989). System identification of human joint dynamics. Critical reviews in biomedical engineering, 18(1):55-87.

Kennedy, W.R., Vanhove, G.F., Lu, S.-p., Tobias, J., Bley, K.R., Walk, D., Wendelschafer-Crabb, G., Simone, D.A., and Selim, M.M. (2010). A randomized, controlled, open-label study of the long-term effects of NGX-4010, a high-concentration capsaicin patch, on epidermal nerve fiber density and sensory function in healthy volunteers. The Journal of Pain, 11(6):579-587.

Khasar, S.G., McCarter, G., and Levine, J.D. (1999). Epinephrine produces a $\beta$-adrenergic receptormediated mechanical hyperalgesia and in vitro sensitization of rat nociceptors. Journal of neurophysiology, 81(3):1104-1112.

Kingdom, F.A.A. and Prins, N. (2010). Psychophysics: a practical introduction. Academic Press London.

Kloeden, P.E. and Pearson, R.A. (1977). The numerical solution of stochastic differential equations. The Journal of the Australian Mathematical Society. Series B. Applied Mathematics, 20(01):812.

Knoblauch, K. and Maloney, L.T. (2012). Modeling psychophysical data in R, volume 32. Springer Science \& Business Media.

Koch, C. and Segev, I. (1998). Methods in neuronal modeling: from ions to networks. MIT press.

Komárek, A. and Lesaffre, E. (2008). Generalized linear mixed model with a penalized gaussian mixture as a random effects distribution. Computational Statistics \& $\mathcal{G}$ Data Analysis, 52(7):34413458 .

Kyranou, M. and Puntillo, K. (2012). The transition from acute to chronic pain: might intensive care unit patients be at risk? Annals of intensive care, 2(1):1-11.

Lapicque, L. (1907). Recherches quantitatives sur l'excitation électrique des nerfs traitée comme une polarisation. J. Physiol. Pathol. Gen, 9(1):620-635.

Latremoliere, A. and Woolf, C.J. (2009). Central sensitization: a generator of pain hypersensitivity by central neural plasticity. The Journal of Pain, 10(9):895-926.

Latremoliere, A. and Woolf, C.J. (2010). Synaptic plasticity and central sensitization: author reply. The Journal of Pain, 11(8):801-803.

Lee, Y.J., Lee, C.H., and Oh, U.T. (2005). Painful channels in sensory neurons. Molecules and Cells.

Lewis, G.N., Luke, H., Rice, D.A., Rome, K., and McNair, P.J. (2012a). Reliability of the conditioned pain modulation paradigm to assess endogenous inhibitory pain pathways. Pain Research ES Management: The Journal of the Canadian Pain Society, 17(2):98.

Lewis, G.N., Rice, D.A., and McNair, P.J. (2012b). Conditioned pain modulation in populations with chronic pain: a systematic review and meta-analysis. The Journal of Pain, 13(10):936-944.

Ljung, L. (1999). System identification(2nd Ed.): Theory for the User. Prentice Hall PTR. 
Ljung, L. and Glad, T. (1994). On global identifiability for arbitrary model parametrizations. Automatica, 30(2):265-276.

Lluch, E., Torres, R., Nijs, J., and Van Oosterwijck, J. (2014). Evidence for central sensitization in patients with osteoarthritis pain: a systematic literature review. European Journal of Pain, 18(10):1367-1375.

Lu, Z.-L. and Dosher, B. (2013). Visual psychophysics: From laboratory to theory. MIT Press.

Luo, C., Kuner, T., and Kuner, R. (2014). Synaptic plasticity in pathological pain. Trends in neurosciences, 37(6):343-355.

Maier, C., Baron, R., Tölle, T.R., Binder, A., Birbaumer, N., Birklein, F., Gierthmühlen, J., Flor, H., Geber, C., Huge, V., et al. (2010). Quantitative sensory testing in the german research network on neuropathic pain (DFNS): somatosensory abnormalities in 1236 patients with different neuropathic pain syndromes. Pain, 150(3):439-450.

Malmberg, A.B. (2000). Protein kinase subtypes involved in injury-induced nociception. In J. Sandkühler, B. Bromm, G. G., editor, Nervous System Plasticity and Chronic Pain, volume 129 of Progress in Brain Research, pages 51 - 59. Elsevier.

Marmarelis, V.Z. (2004). Nonlinear dynamic modeling of physiological systems, volume 10. John Wiley \& Sons.

Martini, C., Olofsen, E., Yassen, A., Aarts, L., and Dahan, A. (2011). Pharmacokineticpharmacodynamic modeling in acute and chronic pain: an overview of the recent literature. Expert review of clinical pharmacology, 4(6):719-728.

Martini, C., Yassen, A., Olofsen, E., Passier, P., Stoker, M., and Dahan, A. (2012). Pharmacodynamic analysis of the analgesic effect of capsaicin 8\% patch (Qutenza ${ }^{\mathrm{TM}}$ ) in diabetic neuropathic pain patients: Detection of distinct response groups. Journal of pain research, 5:51.

McKay, M.D., Beckman, R.J., and Conover, W.J. (2000). A comparison of three methods for selecting values of input variables in the analysis of output from a computer code. Technometrics, 42(1):55-61.

Melzack, R. and Wall, P.D. (1965). Pain mechanisms: a new theory. Science (New York, NY), 150(3699):971-979.

Miao, H., Xia, X., Perelson, A.S., and Wu, H. (2011). On identifiability of nonlinear ode models and applications in viral dynamics. SIAM review, 53(1):3-39.

Millan, M.J. (2002). Descending control of pain. Progress in neurobiology, 66(6):355-474.

Mogyoros, I., Kiernan, M.C., and Burke, D. (1996). Strength-duration properties of human peripheral nerve. Brain, 119(2):439.

Moore, K.A., Baba, H., and Woolf, C.J. (2000). Synaptic transmission and plasticity in the superficial dorsal horn. Progress in brain research, 129:63.

Mørch, C.D., Hennings, K., and Andersen, O.K. (2011). Estimating nerve excitation thresholds to cutaneous electrical stimulation by finite element modeling combined with a stochastic branching nerve fiber model. Medical \&3 biological engineering \&3 computing, 49(4):385-395.

Moscatelli, A., Mezzetti, M., and Lacquaniti, F. (2012). Modeling psychophysical data at the 
population-level: the generalized linear mixed model. Journal of vision, 12(11):26.

Mouraux, A., Iannetti, G.D., and Plaghki, L. (2010). Low intensity intra-epidermal electrical stimulation can activate a $\delta$-nociceptors selectively. Pain, 150(1):199-207.

Mouraux, A., Marot, E., and Legrain, V. (2014). Short trains of intra-epidermal electrical stimulation to elicit reliable behavioral and electrophysiological responses to the selective activation of nociceptors in humans. Neuroscience letters, 561:69-73.

Naugle, K.M., Cruz-Almeida, Y., Vierck, C.J., Mauderli, A.P., and Riley, J.L. (2015). Age-related differences in conditioned pain modulation of sensitizing and desensitizing trends during response dependent stimulation. Behavioural brain research, 289:61-68.

Niederberger, E. and Geisslinger, G. (2008). Proteomics in neuropathic pain research. Anesthesiology, 108(2):314-323.

Nunziata, E., Perez, C., Jarmul, E., Lipetz, L.E., and Weed, H.R. (1989). Effect of tactile stimulation pulse characteristics on sensation threshold and power consumption. Annals of biomedical engineering, 17(4):423-435.

Olesen, A.E., Andresen, T., Staahl, C., and Drewes, A.M. (2012). Human experimental pain models for assessing the therapeutic efficacy of analgesic drugs. Pharmacological reviews, 64(3):722-779.

O'Neill, J., Brock, C., Olesen, A.E., Andresen, T., Nilsson, M., and Dickenson, A.H. (2012). Unravelling the mystery of capsaicin: a tool to understand and treat pain. Pharmacological reviews, 64(4):939-971.

Oron, A.P. and Hoff, P.D. (2009). The k-in-a-row up-and-down design, revisited. Statistics in medicine, 28(13):1805-1820.

Palmer, J., Huk, A.C., and Shadlen, M.N. (2005). The effect of stimulus strength on the speed and accuracy of a perceptual decision. Journal of vision, 5(5):1.

Petersen, K.L. and Rowbotham, M.C. (1999). A new human experimental pain model: the heat/capsaicin sensitization model. Neuroreport, 10(7):1511-1516.

Pfau, D.B., Klein, T., Putzer, D., Pogatzki-Zahn, E.M., Treede, R.-D., and Magerl, W. (2011). Analysis of hyperalgesia time courses in humans after painful electrical high-frequency stimulation identifies a possible transition from early to late ltp-like pain plasticity. Pain, 152(7):15321539 .

Pintelon, R. and Schoukens, J. (2012). System identification: a frequency domain approach. John Wiley \& Sons.

Plesser, H.E. and Gerstner, W. (2000). Noise in integrate-and-fire neurons: From stochastic input to escape rates. Neural Computation, 12(2):367-384.

Polydefkis, M., Hauer, P., Sheth, S., Sirdofsky, M., Griffin, J.W., and McArthur, J.C. (2004). The time course of epidermal nerve fibre regeneration: studies in normal controls and in people with diabetes, with and without neuropathy. Brain, 127(7):1606-1615.

Prescott, S.A. and Koninck, Y.D. (2002). Four cell types with distinctive membrane properties and morphologies in lamina I of the spinal dorsal horn of the adult rat. The Journal of Physiology, 539(3):817-836. 
Pud, D., Granovsky, Y., and Yarnitsky, D. (2009). The methodology of experimentally induced diffuse noxious inhibitory control (DNIC)-like effect in humans. PAIN囚, 144(1):16-19.

Purves, D. (2008). Neuroscience. Number no. 494, p. 2008 in Neuroscience. W. H. Freeman.

Quick Jr, R.F. (1974). A vector-magnitude model of contrast detection. Kybernetik, 16(2):65-67.

Ranger, J. and Kuhn, J. (2014). An accumulator model for responses and response times in tests based on the proportional hazards model. British Journal of Mathematical and Statistical Psychology, 67(3):388-407.

Ratcliff, R. and McKoon, G. (2008). The diffusion decision model: theory and data for two-choice decision tasks. Neural computation, 20(4):873-922.

Ratcliff, R. and Rouder, J.N. (1998). Modeling response times for two-choice decisions. Psychological Science, 9(5):347-356.

Rattay, F. (1999). The basic mechanism for the electrical stimulation of the nervous system. Neuroscience, 89(2):335-346.

Raue, A., Karlsson, J., Saccomani, M.P., Jirstrand, M., and Timmer, J. (2014). Comparison of approaches for parameter identifiability analysis of biological systems. Bioinformatics, page btt006.

Raue, A., Kreutz, C., Maiwald, T., Bachmann, J., Schilling, M., Klingmüller, U., and Timmer, J. (2009). Structural and practical identifiability analysis of partially observed dynamical models by exploiting the profile likelihood. Bioinformatics, 25(15):1923-1929.

Raue, A., Kreutz, C., Theis, F.J., and Timmer, J. (2013). Joining forces of bayesian and frequentist methodology: a study for inference in the presence of non-identifiability. Philosophical Transactions of the Royal Society of London A: Mathematical, Physical and Engineering Sciences, 371(1984):20110544.

Ricciardi, L.M. and Sato, S. (1986). On the evaluation of first passage time densities for gaussian processes. Signal Processing, 11(4):339-357.

Rolke, R., Baron, R., Maier, C., Tölle, T.R., Treede, R.-D., Beyer, A., Binder, A., Birbaumer, N., Birklein, F., Bötefür, I.C., et al. (2006). Quantitative sensory testing in the German Research Network on Neuropathic Pain (DFNS): standardized protocol and reference values. Pain, 123(3):231-243.

Roth, A. and van Rossum, M.C.W. (2009). Modeling synapses. In De Schutter, E., editor, Computational Modeling Methods for Neuroscientists, pages 139-160. The MIT Press.

Roy, B.K. and Smith, D.R. (1969). Analysis of the exponential decay model of the neuron showing frequency threshold effects. The Bulletin of mathematical biophysics, 31(2):341-357.

Ruscheweyh, R., Wilder-Smith, O.H.G., Drdla, R., Liu, X.-G., and Sandkühler, J. (2011). Longterm potentiation in spinal nociceptive pathways as a novel target for pain therapy. Mol Pain, $7(1): 20$.

Saccomani, M.P. and Cobelli, C. (1993). A minimal input-output configuration for a priori identifiability of a compartmental model of leucine metabolism. Biomedical Engineering, IEEE Transactions on, 40(8):797-803. 
Sandkühler, J. (2007). Understanding LTP in pain pathways. Mol Pain, 3(9).

Sandkühler, J. (2009). Models and mechanisms of hyperalgesia and allodynia. Physiological reviews, $89(2): 707-758$.

Sandkühler, J. (2010). Central sensitization versus synaptic long-term potentiation (LTP): a critical comment. The Journal of Pain, 11(8):798-800.

Schmelz, M. and Kress, M. (1996). Topical acetylsalicylate attenuates capsaicin induced pain, flare and allodynia but not thermal hyperalgesia. Neuroscience letters, 214(1):72-74.

Schmidt, P.J.. (2015). Norovirus dose-response: Are currently available data informative enough to determine how susceptible humans are to infection from a single virus? Risk Analysis, 35(7):1364-1383.

Sinke, C., Schmidt, K., Forkmann, K., and Bingel, U. (2015). Phasic and tonic pain differentially impact the interruptive function of pain. PloS one, 10(2):e0118363.

Srivastava, V., Feng, S.F., Cohen, J.D., Leonard, N.E., and Shenhav, A. (2015). First passage time properties for time-varying diffusion models: A martingale approach. arXiv preprint arXiv:1508.03373.

Steenbergen, P., Buitenweg, J.R., Trojan, J., van der Heide, E.M., van den Heuvel, T., Flor, H., and Veltink, P.H. (2012). A system for inducing concurrent tactile and nociceptive sensations at the same site using electrocutaneous stimulation. Behavior Research Methods, 44(4):924-933.

Steiert, B., Raue, A., Timmer, J., and Kreutz, C. (2012). Experimental design for parameter estimation of gene regulatory networks. PloS one, 7(7):e40052.

Stone, J.V. (2014). Using reaction times and binary responses to estimate psychophysical performance: an information theoretic analysis. Frontiers in neuroscience, 8.

Stüttgen, M.C., Schwarz, C., and Jäkel, F. (2011). Mapping spikes to sensations. Frontiers in neuroscience, 5 .

Szallasi, A. (2010). Analgesia: Methods and Protocols. Methods in Molecular Biology. Humana Press.

Todd, A.J. (2010). Neuronal circuitry for pain processing in the dorsal horn. Nature Reviews Neuroscience, 11(12):823-836.

Tolner, E. A., Houben, T., Terwindt, G.M., de Vries, B., Ferrari, M.D., and van den Maagdenberg, A.M.J.M. (2015). From migraine genes to mechanisms. Pain, 156:S64-S74.

Treede, R. (2012). Peripheral and central mechanisms of neuropathic pain. In Simpson, McArthur, and Dworkinn, editors, Neuropathic Pain: Mechanisms, Diagnosis and Treatment, pages 14-24. Oxford University Press, New York, Oxford.

Treutwein, B. (1995). Adaptive psychophysical procedures. Vision research, 35(17):2503-2522.

Tsang, A., Von Korff, M., Lee, S., Alonso, J., Karam, E., Angermeyer, M. C., Borges, G. L. G., Bromet, E. J., De Girolamo, G., De Graaf, R., et al. (2008). Common chronic pain conditions in developed and developing countries: gender and age differences and comorbidity with depressionanxiety disorders. The Journal of Pain, 9(10):883-891.

Tyler, C.W. and Chen, C.-C. (2000). Signal detection theory in the 2afc paradigm: Attention, 
channel uncertainty and probability summation. Vision research, 40(22):3121-3144.

Uusipaikka, E. (2008). Confidence intervals in generalized regression models. CRC Press.

van der Heide, E. (2009). Neurophysiological observation of the nociceptive system using electrocutaneous stimulation. PhD thesis, University of Twente, Enschede.

van der Heide, E.M., Buitenweg, J.R., Marani, E., and Rutten, W.L.C. (2009). Single pulse and pulse train modulation of cutaneous electrical stimulation: a comparison of methods. Journal of Clinical Neurophysiology, 26(1):54-60.

van Mourik, S., ter Braak, C., Stigter, H., and Molenaar, J. (2014). Prediction uncertainty assessment of a systems biology model requires a sample of the full probability distribution of its parameters. PeerJ, 2:e433.

van Riel, N.A.W. (2006). Dynamic modelling and analysis of biochemical networks: mechanismbased models and model-based experiments. Briefings in bioinformatics, 7(4):364-374.

Vaneker, M., Wilder-Smith, O.H.G., Schrombges, P., de Man-Hermsen, I., and Oerlemans, H.M. (2005). Patients initially diagnosed as 'warm' or 'cold' CRPS 1 show differences in central sensory processing some eight years after diagnosis: a quantitative sensory testing study. Pain, 115(1):204-211.

Vanlier, J., Tiemann, C.A., Hilbers, P.A.J., and van Riel, N.A.W. (2013). Parameter uncertainty in biochemical models described by ordinary differential equations. Mathematical biosciences, $246(2): 305-314$.

Viemeister, N.F. and Wakefield, G.H. (1991). Temporal integration and multiple looks. The Journal of the Acoustical Society of America, 90(2):858-865.

Voscopoulos, C. and Lema, M. (2010). When does acute pain become chronic? British journal of anaesthesia, 105(suppl 1):i69-i85.

Walk, D., Sehgal, N., Moeller-Bertram, T., Edwards, R.R., Wasan, A., Wallace, M., Irving, G., Argoff, C., and Backonja, M.-M. (2009). Quantitative sensory testing and mapping: a review of nonautomated quantitative methods for examination of the patient with neuropathic pain. The Clinical journal of pain, 25(7):632-640.

Wall, P. D. and Melzack, R. (1989). Textbook of pain. Churchill Livingstone.

Wang, L.Y., Yin, G.G., Zhang, J., and Zhao, Y. (2010). System identification with quantized observations. Springer.

Watson, A.B. (1979). Probability summation over time. Vision research, 19(5):515-522.

Weng, H.R., Chen, J.H., and Cata, J.P. (2006). Inhibition of glutamate uptake in the spinal cord induces hyperalgesia and increased responses of spinal dorsal horn neurons to peripheral afferent stimulation. Neuroscience, 138(4):1351-1360.

Westwick, D.T. and Kearney, R.E. (2003). Identification of nonlinear physiological systems, volume 7. John Wiley \& Sons.

Wetherill, G.B. (1963). Sequential estimation of quantal response curves. Journal of the Royal Statistical Society. Series B (Methodological), 25(1):1-48.

Wilder-Smith, O.H.G. (2002). Monitoring nociceptive neuroplasticity: quantitative sensory testing: 
a better therapeutic endpoint for managing the pain of surgery? $\mathrm{PhD}$ thesis, Radboud University Nijmegen, Nijmgen.

Wilder-Smith, O.H.G. and Arendt-Nielsen, L. (2006). Postoperative hyperalgesia: its clinical importance and relevance. Anesthesiology, 104(3):601-607.

Wilder-Smith, O.H.G., Tassonyi, E., Crul, B.J., and Arendt-Nielsen, L. (2003). Quantitative sensory testing and human surgery: effects of analgesic management on postoperative neuroplasticity. Anesthesiology, 98(5):1214-1222.

Woolf, C.J. (2011). Central sensitization: implications for the diagnosis and treatment of pain. Pain, 152(3):S2-S15.

Woolf, C.J. and Mannion, R.J. (1999). Neuropathic pain: aetiology, symptoms, mechanisms, and management. The lancet, 353(9168):1959-1964.

Woolf, C.J. and Max, M.B. (2001). Mechanism-based pain diagnosis: issues for analgesic drug development. Anesthesiology, 95(1):241-249.

Woolf, C.J. and Salter, M.W. (2000). Neuronal plasticity: increasing the gain in pain. Science, 288(5472):1765-1768.

Woolf, C.J. and Thompson, S.W.N. (1991). The induction and maintenance of central sensitization is dependent on n-methyl-d-aspartic acid receptor activation; implications for the treatment of post-injury pain hypersensitivity states. Pain, 44(3):293-299.

Xu, F., Lu, T., and Seffen, K. (2008a). Skin thermal pain modeling-a holistic method. Journal of Thermal Biology, 33(4):223-237.

Xu, F., Wen, T., Lu, T.J., and Seffen, K.A. (2008b). Modeling of nociceptor transduction in skin thermal pain sensation. Journal of biomechanical engineering, 130(4):041013.

Yang, H., Feng, Y., and Xu, X.S. (2014). Pharmacokinetic and pharmacodynamic modeling for acute and chronic pain drug assessment. Expert opinion on drug metabolism $\&$ toxicology, 10(2):229-248.

Yang, H., Meijer, H.G.E., Doll, R.J., Buitenweg, J.R., and van Gils, S.A. (2015a). Computational modeling of Adelta-fiber-mediated nociceptive detection of electrocutaneous stimulation. Biological Cybernetics, 109(4):479-491.

Yang, H., Meijer, H.G.E., Doll, R.J., Buitenweg, J.R., and van Gils, S.A. (2015b). Dependence of nociceptive detection thresholds on physiological parameters and capsaicin-induced neuroplasticity: a computational study. Submitted.

Yang, H., Meijer, H.G.E., Buitenweg, J.R., and van Gils, S.A. (2015c). Estimation and identifiability of model parameters in human nociceptive processing using stimulus-response pairs. Submitted.

Yang, H., Meijer, H.G.E., Buitenweg, J.R., and van Gils, S.A. (2015d). Threshold estimates from logistic regression on stimulus-response pairs from staircase procedures with a case study of a nociceptive detection task. In preparation.

Yarnitsky, D. (2010). Conditioned pain modulation (the diffuse noxious inhibitory control-like effect): its relevance for acute and chronic pain states. Current Opinion in Anesthesiology, 23(5):611-615. 
Yarnitsky, D., Arendt-Nielsen, L., Bouhassira, D., Edwards, R.R., Fillingim, R.B., Granot, M., Hansson, P., Lautenbacher, S., Marchand, S., and Wilder-Smith, O.H.G. (2010). Recommendations on terminology and practice of psychophysical dnic testing. European Journal of Pain, 14(4):339-339.

Zhao, Y., Wang, L.Y., Yin, G.G., and Zhang, J.-F. (2007). Identification of wiener systems with binary-valued output observations. Automatica, 43(10):1752-1765.

Zwislocki, J. (1960). Theory of temporal auditory summation. The Journal of the Acoustical Society of America, 32(8):1046-1060. 


\section{Summary}

Efficient treatment for pain disorders could benefit from improved diagnostic tools by identifying defective nociceptive processes. To develop such a tool, this thesis follows a system approach by building plausible computational models that characterize essential peripheral and central subsystems. To offer a mechanism-based interpretation, we perform mathematical analysis on these models. In particular, we use psychophysical measurements from human subjects to perform parameter estimation and identifiability analysis.

In Chapter 2, we have built neurophysiology-based models, representing both peripheral and central nociceptive subsystems for a nociceptive detection task. Our computational modeling does not only account for neural integration but also the principle of probability summation. In addition, we incorporate psychophysical observations of detection thresholds for a qualitative validation. For normal nociceptive function, results in Chapter 2 show qualitative agreement between model-simulated and experimentally obtained detection thresholds regarding the dependence of the threshold on temporal stimulus properties: the number of pulses, the pulse width, and the interpulse interval. In turn, it provides explanatory insights into the underlying mechanisms. In particular, our analysis in Chapter 3 reveals that the observed dependence of thresholds on the interpulse interval could be attributed to specific properties in central nociceptive subsystems.

With the developed hazard model, we investigate nociceptive neuroplasticity. First of all, Chapter 3 investigates how different forms of neuroplasticity affect detection thresholds by sweeping model parameters. Second, model simulations indicate how different forms of capsaicin-induced plasticity could affect the A-fibermediated nociceptive function. Especially, simulation results suggest that capsaicininduced central neuroplasticity can last over a month. This finding is in line with the suspected central plasticity which was recently reported in an experimental pain model using high-frequency electrical stimulation from an other research group. Indeed, our model-based study (Chapter 3) is the first to suggest a long-lasting central plasticity upon an application of a high-dose capsaicin patch. This hypothesisgenerating study also suggests other interventions together with capsaicin application to further explore and validate capsaicin-induced effects. 
In Chapter 4, we address estimation and identifiability of parameters in the hazard model with stimulus-response pairs measured from individual subjects. First, for a structurally identifiable model, a necessary condition on temporal stimulus properties follows from our analysis. Also, we provide both analytic and numerically implemented assessments of the set identifiability, when only one single value for the pulse width is used within the stimulus set. Here, our results indicate that the larger the pulse width is, the narrower the set identifiability is. Second, our study demonstrates the applicability of profile likelihood to assess identifiability in a (nonlinear) physiology-based model using psychophysical measurements. We also observed that existing data from a ten-minute detection task could hamper parameter identifiability. In order to improve parameter identifiability, we performed a model-based study to choose stimulus properties. Further applications of our approach might facilitate mechanism-based differential diagnosis of malfunctioning nociceptive processes.

In Chapter 5, we formulate the staircase procedures utilized in the experimental detection task as Markov models. Our Markovian formulation of staircase procedures (including the recently developed adaptive probing procedure) facilitates characterization of (asymptotic) distributions of sampled amplitudes of applied stimuli. Next, together with simulated stimulus-response pairs using the hazard model, we evaluate the estimation performance of detection thresholds from logistic regression. Both our theoretical analysis and simulations reveal the existence of a bias in the threshold estimate. This finding also suggests that in order to estimate parameters in the hazard model, one should use measured stimulus-response pairs (Chapter 4) rather than intermediate estimates of detection thresholds.

Chapter 6 emphasizes contributions of this research to advance our understanding of nociceptive processing. With parameters characterizing peripheral and central nociceptive subsystems, we highlight our model-based framework with a promising potential to offer mechanism-based diagnosis for pain disorder patients. With respect to computational modeling and analysis, we point out prospective research directions including modeling of other relevant nociceptive processes as well as possible integration with other psychophysical measurements. We discuss how possible usage of the developed computational tools might advance our understanding of pain disorders towards clinical diagnosis to differentiate malfunctioning subsystems. 


\section{Samenvatting}

De behandeling van pijn symptomen zou profijt kunnen hebben van een diagnostieke tool om het onderliggende gebrekkig functionerende nociceptieve systeem te identificeren. Om zo'n tool te ontwikkelen richt dit proefschrift zich op een systeem aanpak waarbij biologisch aannemelijke wiskundige modellen worden gemaakt die essentiële eigenschappen van perifere en centrale zenuwstelsel meenemen. Door middel van een wiskundige analyse van deze modellen komen we tot een interpretatie die gebaseerd is op onderliggende processen. In het bijzonder gebruiken we data van psychofysische metingen bij gezonde proefpersonen om parameters te schatten en de identificeerbaarheid van de modellen te bepalen.

In Hoofdstuk 2 hebben we een wiskundig model gemaakt van een nociceptieve detectie taak. Het model representeert de neurofysiologie van het perifere en centrale zenuwstelsel. Het model neemt niet alleen neurale integratie van signalen mee, maar ook het sommeren van kansen. We gebruiken psychofysische meetdata van waarnemingsdrempels om het model kwalitatief te valideren. De resultaten in dit hoofdstuk laten in het geval van een gezond functionerend systeem een kwalitatieve overeenkomst zien hoe de waarnemingsdrempel afhangt van de variatie in de experimentele stimulus parameters: het aantal pulsen, de pulsbreedte en de tijd tussen de pulsen. Daarbij leren we ook iets over de onderliggende processen. In het bijzonder tonen we in Hoofdstuk 3 aan dat specifieke eigenschappen van het centrale systeem bepalend zijn voor de functionele relatie tussen waarnemingsdrempel en de tijd tussen de pulsen.

In Hoofdstuk 3 bestuderen we plasticiteit in het nociceptieve systeem aan de hand van het ontwikkelde hazard model. We bepalen hoe neuroplasticiteit de waarnemingsdrempels kan beïnvloeden door eerst parameters te variëren. Vervolgens bestuderen we verschillende vormen van neuroplasticiteit veroorzaakt door capsaïcine. We laten met model simulaties zien hoe de waarnemingsdrempels veranderen. De simulaties suggereren in het bijzonder dat capsaïcine een langdurig, d.w.z. minstens een maand, plasticiteitseffect kan hebben op centraal niveau. Dit resultaat sluit aan bij eerdere experimentele resultaten van een andere groep waar hoog frequente stimulatie werd gebruikt. Onze resultaten zijn de eerste die wijzen op een dusdanig langdurig effect van een toepassing van hoge dosis capsaïcine patch. 
Dit suggereert ook andere klinische interventies in combinatie met toepassen van capsaïcine te verkennen en te valideren.

In Hoofdstuk 4 richten we ons op het schatten en de identificeerbaarheid van parameters in het hazard model door middel van stimulus-responsie data van individuele proefpersonen. Onze analyse geeft als eerste een noodzakelijke voorwaarde voor structurele identificeerbaarheid in termen van de temporele stimulus eigenschappen. Vervolgens laten we analytisch en numeriek de gevolgen zien voor de identificeerbaarheid als slechts een pulsbreedte gebruikt wordt. We vinden dat een langere pulsduur de [set-]identificeerbaarheid verbetert. Ten tweede laten we zien dat de profile-likelihood methode met stimulus-responsie data geschikt is om de praktische identificeerbaarheid van het model te onderzoeken. We merken daarbij op dat de data van de tien minuten durende detectie taak soms te beperkte informatie bevat. Om de parameter identificeerbaarheid te verbeteren hebben we onderzocht hoe de temporele stimulus eigenschappen gekozen moeten worden. Een verdere ontwikkeling van deze aanpak zou kunnen leiden tot een tool om een diagnose van een slecht functionerend nociceptief proces te stellen op grond van onderliggende mechanismen.

Bij de detectie taak wordt een "staircase"-algoritme gebruikt om de stimulus amplitude te variëren. In Hoofdstuk 5 formuleren we deze procedure, inclusief de recent voorgestelde "adaptive probing" procedure, als een Markov model. Dit maakt het mogelijk om de (asymptotisch) distributies van de gebruikte stimulus amplitudes te bestuderen. Samen met stimulus-responsie data op basis van het model, onderzoeken we hiermee hoe goed de waarnemingsdrempels geschat worden met logistische regressie. We vinden zowel asymptotisch als uit de model-data dat er een bias bestaat in de schatting van de drempel. Dit resultaat laat zien dat om parameters in het hazard model te schatten het beter is om direct de stimulusresponsie data te gebruiken in plaats van de waarnemingsdrempels.

Hoofdstuk 6 benadrukt de bijdragen van dit onderzoek aan een verbeterd begrip van het nociceptieve systeem. Ons model heeft parameters die perifere en centrale nociceptieve deelsystemen karakteriseren. Dit biedt perspectief op de lange termijn om tot een diagnostiek op basis van onderliggende mechanismen te komen voor pijnpatiënten. We beschrijven enkele aspecten die in verder theoretisch, modelleer en computationeel werk onderzocht kunnen worden, zoals het meenemen van meer nociceptieve processen en het gebruik van andere psychofysische data. 


\section{Acknowledgments}

First of all, I would like to express my deepest gratitude to my promoter, Stephan van Gils. I thank for your interests in my research. Moreover, I appreciate a lot for your persistent encouragements to me during the stressful period of thesis writing. Stephan, thank you for being a positive influence on my research trajectory over years towards the completion of this thesis.

Secondly, I thank my co-promoters, Hil Meijer and Jan Buitenweg, for your enthusiasm and dedication to my research. Hil, thanks a lot for your daily supervision, which is much more than our regular meetings on Tuesdays. I greatly appreciate your insightful suggestions to my work in many aspects, and also patience and guidance to help me organize my thoughts into papers. Moreover, thanks for your flexibility and openness to allow me to perform a 'random walk' study, which finally converged to those Markov models (Chapter 5). Jan, thanks for your inputs in our project meetings and efforts to our lengthy discussions on manuscripts. Your coordination of the PAINSIGHT project played an important role for the completion of my doctoral research. I think that effect is more than a probability summation. Stephan, Hil and Jan, I really appreciate those meetings with all of you, which definitely sped up my thesis writing.

I thank Peter Veltink (UTwente), Michel van Putten (UTwente), Dieter Kleinböhl (Universität Mannheim), Alfred Schouten (TU Delft), and Richard Boucherie (UTwente) for being my doctoral thesis committee and reading my thesis. I also thank Hans Zwart (UTwente) for agreeing to chair my defense.

Working in a multidisciplinary project, I appreciate helps from collaborators, who make this thesis possible. I thank Robert-Jan for your extensive collaboration. Robert-Jan, I enjoy our $(1+1>2)$-style presentations to audiences with various backgrounds and I like engaging discussions about statistics with you. Many thanks also go to the user committee of the PAINSIGHT for expressing interests and providing valuable feedback to my research. I thank Dieter for organizing the theme section in Fechner Day 2013 (together with Jan and Hil), which advocated system identification approaches in psychophysics. I thank Justin Hay from the Centre for Human Drug Research (CHDR) in Leiden for sharing your expertise in human experimental pain models. I also thank Jan Freijer and Jasper Stevens from the CHDR 
for introducing me with Pharmacokinetic/Pharmacodynamic modeling techniques. In addition, I acknowledge support from the Dutch Technology Foundation STW to my research.

As my doctoral research was mainly conducted in the Department of Applied Mathematics, many colleagues there helped me in many ways, which made it smoother to complete my thesis. Thanks go to Marielle and Linda for your assistance in many administrative issues. I would also like to thank other members from the Applied Analysis group: Bettina, Koen, Jurgen, Leonie, Christoph, Mike and also former colleagues: Marcel, Sid and Antonios for research assistance and inspiring group meetings. In addition, I thank other peers from the SACS: Lulu, Tatyana, Deepak, Ruddy, Didit, Wenny, Edo, Felix, Poorvi, Sjoerd, Wilbert, Gijs, Paolo and others, who create a stimulating work environment.

I thank Yong, Dongshuang, Binlong, Haishan, Changxin, Kenan, Zhemin, Zhao, Peng for your continuous support and care in Twente. I thank Xiaoyan Zhang for lunch walks in Twente campus.

Finally, I am greatly indebted to my parents, Yang Jianzhong and Che Yunhua for your endless love and support, whenever and wherever I need. I am thankful to Ajing and Walter for your kindness and care. Fanlu, I am grateful to your unconditional love and accompany during not only our happy moments but also the strenuous time.

Huan Yang,

Enschede, November 2015. 


\section{About the Author}

Huan Yang was born on December 29th, 1985 at Liaoyang, China. He stayed in his hometown until his undergraduate study of Biomedical Engineering in 2004 at Northeastern University, Shenyang, China. In his graduation project, he prototyped an image acquisition system for Cone beam CT scans with a X-ray flat panel detector. In 2009, he received the Talented Student Scholarship for a two-year Master program of Biomedical Engineering at the Technology University of Eindhoven in the Netherlands. This study experience offered him a foundation of computational modeling in systems biology. He completed three traineeships on topics of radiotherapy, biomedical image analysis, and parameter sensitivity analysis in the group of BioModeling and BioInformatics, Eindhoven and the Maastro Clinic, Maastricht. After earning his master's degree in 2011, he joined the group of Applied Analysis in the University of Twente as a mathematical modeler to pursue a doctoral degree. In the multidisciplinary project (NeuroSIPE-PAINSIGHT) to gain insight into the development of pain disorders, he was mainly responsible for developing computational tools to represent and to further understand underlying nociceptive processes. The majority of his doctoral research was presented in this book. 


\section{List of Publication}

\section{Journal articles}

Yang H., Meijer H.G.E., Doll R.J., Buitenweg, J.R., and van Gils S.A. (2015), Computational modeling of Adelta-fiber-mediated nociceptive detection of electrocutaneous stimulation, Biological Cybernetics, Volume 149, Issue 4, Page 479-491. doi:10.1007/s00422015-0656-4.

Yang H., Meijer H.G.E., Doll R.J., Buitenweg J.R., and van Gils S.A. (2015), Dependence of nociceptive detection thresholds on physiological parameters and capsaicin-induced neuroplasticity: a computational study, Submitted.

Yang H., Meijer H.G.E., Buitenweg J.R., and van Gils S.A. (2015), Estimation and identifiability of model parameters in human nociceptive processing using stimulus-response pairs, Submitted.

Yang H., Meijer H.G.E., Buitenweg J.R., and van Gils S.A. (2015), Threshold estimates from logistic regression on stimulus-response pairs from staircase procedures with a case study of a nociceptive detection task, In preparation. 


\section{Conference contributions}

Yang H., Meijer H.G.E., Doll R.J., Buitenweg J.R., and van Gils S.A., Capsaicininduced neuroplasticity on human nociceptive function: a model-based study, 5th Dutch Bio-Medical Engineering Conference 2015, Egmond aan Zee, the Netherlands, January 2015 (talk)

Yang H., Buitenweg J.R., and Meijer H.G.E., System identification of nociceptive function, 29th Annual Meeting of the International Society for Psychophysics, Freiburg im Breisgau, Germany, October 2013 (talk).

Yang H., Buitenweg J.R., and Meijer H.G.E., Electrocutaneous stimulus setting for identification of the ascending nociceptive pathway, 22nd Annual Computational Neuroscience Meeting, Paris, France, July 2013 (poster). doi:10.1186/1471-2202-14-S1-P272.

Yang H., Buitenweg J.R., and Meijer H.G.E., Identification of the nociceptive forward pathway using amplitude-response pairs, 4th Dutch Bio-Medical Engineering Conference 2013, Egmond aan Zee, the Netherlands, January 2013 (poster).

Yang H., Buitenweg J.R., and Meijer H.G.E., Identification of the nociceptive forward pathway using perception thresholds, 8th International Federation of Automatic Control (IFAC) Symposium on Biological and Medical Systems, Budapest, Hungary, August 2012 (talk). doi:10.3182/20120829-3-HU-2029.00103.

Yang H., Buitenweg J.R., and Meijer H.G.E., Identification of the ascending sensory pathway using perception thresholds, PEDS-II Workshop, Eindhoven, the Netherlands, June 2012 (poster).

Yang H., Doll R.J., Meijer H.G.E., and Buitenweg J.R., Modeling and identifying the ascending sensory system with the psychophysical binary Responses and electrocutaneous stimuli, Joint meeting: 10th National Day on Biomedical engineering and Annual symposium of the IEEE EMBS Benelux Chapter, Leuven, Belgium, December 2011 (poster).

Doll R.J., Yang H., Meijer H.G.E., and Buitenweg J.R., Simulation of psychophysical stimulus selection procedures for dynamic threshold tracking, Joint meeting: 10th National Day on Biomedical engineering and Annual symposium of the IEEE EMBS Benelux Chapter, Leuven, Belgium, December 2011 (poster).

Yang H., Vanlier J., Hilbers P.A.J., and van Riel N.A.W., Comparative study of global parameter sensitivity analyses of models based on ordinary differential equations, 3rd Dutch Bio-Medical Engineering Conference 2011, Egmond aan Zee, the Netherlands, January 2011 (poster). 